\title{
Control of molecular rotation with an optical centrifuge
}

\author{
by \\ Aleksey Korobenko
}

B.Sc., Moscow Institute of Physics and Technology, 2008

M.Sc., Moscow Institute of Physics and Technology, 2010

A THESIS SUBMITTED IN PARTIAL FULFILLMENT OF

THE REQUIREMENTS FOR THE DEGREE OF

DOCTOR OF PHILOSOPHY

in

The Faculty of Graduate and Postdoctoral Studies

(Physics)

THE UNIVERSITY OF BRITISH COLUMBIA

(Vancouver)

July 2016

(C) Aleksey Korobenko 2016 


\section{Abstract}

The major purpose of this work is the experimental study of the applicability of an optical centrifuge - a novel tool, utilizing non-resonant broadband laser radiation to excite molecular rotation - to produce and control molecules in extremely high rotational states, so called molecular "super rotors", and to study their optical, magnetic, acoustic, hydrodynamic and quantum mechanical properties. 


\section{Preface}

All the work presented in this thesis was conducted using the optical centrifuge setup, discussed in section 2.1. I was heavily involved in the design and construction of the centrifuge together with Gilad Hurvitz, Alexander Milner and Valery Milner. I also actively participated in the development of general methods of optical characterization of centrifuge pulses. Among them, I conceived and implemented the method of determining an absolute angle of the centrifuge field, described in subsection 2.4.2. The ultra-high vacuum chamber and the velocity map imaging setup, described in sections 2.3.1 and 2.4 respectively, were designed together with Valery Milner and built by me. I also helped to design and construct the gas chamber described in subsection 2.2.1 and used for the investigation of super rotors under ambient conditions.

I was heavily involved in designing and interpreting the results of experiments on the first direct observation of oxygen and nitrogen super rotors, based on coherent Raman spectroscopy, developed and implemented together with Alexander Milner and Valery Milner. These results were published in Paper 1 and discussed in Section 3.1 and Section 4.1.

After setting up the tunable dye laser system together with Alice SchmidtMay, I became the primary investigator in the area of resonance-enhanced multi-photon ionization (REMPI) spectroscopy of centrifuged oxygen. Under the guidance of Valery Milner and John Hepburn, I designed the experiment, collected data and interpreted the results, which were published in Paper 2 and discussed in Section 3.2 .

I have designed, implemented and continuously improved the experimental setup for the direct imaging of molecular super rotors by means of velocity map imaging (VMI). Using the VMI technique, I collected the data on dispersionless "stopwatch" rotational states, as well as "cogwheel" states, described in Paper 5 and Section 4.2, I also used the same VMI method to study the properties of centrifuged asymmetric top molecules, collecting and analysing the data published in Paper 11 (Section 5.3). Finally, I modified and applied the ion imaging system to investigate the properties of non-magnetic and paramagnetic super rotors in external magnetic fields. 
The results of this studies, based on my experimental data and numerical analysis, were published in Paper 8 and discussed in Section 5.2.

Magneto-optic properties of molecular super rotors, described in Paper 7, were studied in collaboration with the theory group of Ilya Averbukh from the Weizmann Institute of Science. The majority of the experimental data for this work was collected by Alexander Milner, whereas my contribution amounted to the data analysis and to providing an important VMI image in support of the main claim of the paper, specifically, the visualization of the magnetic splitting of the angular distribution of oxygen super rotors in an external magnetic field (Section 5.2).

The work on the collisional relaxation of molecular super rotors was spearheaded by Alexander Milner, who collected the majority of the experimental data using the technique of coherent Raman scattering. Rotational decoherence of non-magnetic nitrogen and magnetic oxygen was discussed in Papers 3 and 4, respectively (Section 6.1). My contribution to this work consisted in proposing the experimental configurations and analyzing the collected data. I provided similar contribution to the work on the collisional re-orientation (Paper 9, Section 6.2) and sound generation (Paper 6, Section 6.4) in dense ensembles of molecular super rotors. The idea of a "two-dimensional" (2D) centrifuge and its effect on the molecular alignment, described in Section 6.3, was conceived with my direct input. To Paper 10, describing the results of this work, I contributed by supplementing the data of Alexander Milner, based on the laser-induced optical birefringence, by collecting and interpreting the VMI images of aligned molecules exposed to the field of a $2 \mathrm{D}$ centrifuge.

I participated in writing all the 11 manuscripts, which I co-authored. They are listed below.

1. Korobenko A., Milner A. A., Milner V., Complete control, direct observation and study of molecular superrotors. Phys. Rev. Lett., 112, 113004 (2014).

2. Korobenko A., Milner A. A., Hepburn J.W., Milner V., Rotational spectroscopy with an optical centrifuge. Phys. Chem. Chem. Phys., 16, 4071 (2014).

3. Milner A. A., Korobenko A., Hepburn J.W., Milner V., Effects of ultrafast molecular rotation on collisional decoherence. Phys. Rev. Lett., 113, 043005 (2014).

4. Milner A. A., Korobenko A., Milner V., Coherent spin-rotational dynamics of oxygen superrotors. New J. Phys. 16, 093038 (2014). 
5. Korobenko A., Hepburn J.W., Milner V., Observation of nondispersing classical-like molecular rotation. Phys. Chem. Chem. Phys. 17, 951 (2015).

6. Milner A. A., Korobenko A., Milner V., Sound emission from the gas of molecular superrotors. Opt. Express 23, 8603 (2015).

7. Milner A. A., Korobenko A., Floss J., Averbukh I. Sh., Milner V., Magneto-optical properties of paramagnetic superrotors. Phys. Rev. Lett., 115, 033005 (2015).

8. Korobenko A., Milner V., Dynamics of molecular superrotors in external magnetic field. J. Phys. B. 48, 164004 (2015).

9. Milner A. A., Korobenko A., Rezaiezadeh K., Milner V., From gyroscopic to thermal motion: a crossover in the dynamics of molecular superrotors. Phys. Rev. X 5, 031041 (2015).

10. Milner A. A., Korobenko A., Milner V., Field-free long-lived alignment of molecules in extreme rotational states. Phys. Rev. A 93, 053408 (2016).

11. Korobenko A., Milner V., Adiabatic field-free alignment of asymmetric top molecules with an optical centrifuge. Phys. Rev. Lett. 116, 183001 (2016). 


\section{Table of Contents}

Abstract $\ldots \ldots \ldots \ldots \ldots \ldots \ldots \ldots$ ii

Preface $\ldots \ldots \ldots \ldots \ldots \ldots \ldots \ldots \ldots \ldots$ iii

Table of Contents . . . . . . . . . . . . . vi

List of Tables . . . . . . . . . . . . . . . viii

List of Figures $\ldots \ldots \ldots \ldots \ldots \ldots \ldots \ldots$

Acknowledgements . . . . . . . . . . . . xxiv

1 Introduction . . . . . . . . . . . . . . . 1

1.1 Rotational motion of molecules . . . . . . . . . . . . 3

1.2 Control of rotational states with intense non-resonant laser

fields . . . . . . . . . . . . . . . . 4 4

1.3 Optical centrifuge . . . . . . . . . . . . . . 8

1.4 Goals of this work . . . . . . . . . . . . . . 11

2 Experimental methods . . . . . . . . . . . . . 15

2.1 Optical centrifuge $\ldots \ldots \ldots \ldots \ldots \ldots \ldots$

2.1 .1 Laser system . . . . . . . . . . . . . . . . . . . 16

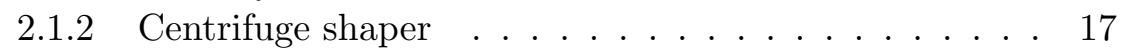

2.2 Raman setup . . . . . . . . . . . . . . . . . . 22

2.2 .1 Raman chamber . . . . . . . . . . . . . . . 23

2.2.2 Multi-pass amplifier . . . . . . . . . . . . . 24 24

2.2 .3 Broadband probe beam . . . . . . . . . . . . . 26

2.2.4 Rotational Raman spectroscopy . . . . . . . . . 27 27

2.3 Resonance-enhanced multiphoton ionization spectroscopy . . 29

2.3.1 Utra-high vacuum chamber and molecular source . . 30

2.3.2 Time-of-flight spectrometer . . . . . . . . . . . . . . 32

2.3 .3 Dye laser system . . . . . . . . . . . . 35 
2.3.4 REMPI spectroscopy of $\mathrm{N}_{2}$ ant $\mathrm{O}_{2} \ldots \ldots \ldots$

2.4 Velocity-map imaging $(\mathrm{VMI}) \ldots \ldots \ldots$

2.4.1 Velocity map imaging reconstruction of the angular distribution ................ 43

2.4.2 Centrifuge angle measurement . . . . . . . . 45

3 Direct detection of molecular super rotors . . . . . . . . . . 48

3.1 Raman spectroscopy of molecular super rotors . . . . . . . 49

3.2 Rotational spectroscopy with an optical centrifuge . . . . . 52

3.3 Mapping out the angular distribution of molecular super rotors ....................... 58

4 Rotational dynamics of molecular super rotors . . . . . . 63

4.1 Rotational revivals in the ensembles of molecular super rotors 63

4.2 Observation of classical-like molecular rotation . . . . . . 65 65

4.3 Coherent spin-rotational dynamics in oxygen . . . . . . . 73

4.4 Dynamics of super rotors in external magnetic fields . . . . 77

5 Molecular alignment with an optical centrifuge . . . . . . 85

5.1 Two-dimensional optical centrifuge . . . . . . . . 86

5.2 Magneto-optical properties of paramagnetic super rotors . . 93

5.3 Adiabatic alignment of asymmetric top molecules . . . . . 99

6 Collisional decay of rotational excitation in dense media . 108

6.1 Effects of ultrafast rotation on collisional decoherence . . . . 109

6.2 Crossover from gyroscopic to thermal motion . . . . . . . . . 117

6.3 Long-lived permanent molecular alignment . . . . . . . . . . 126

6.4 Sound emission from the gas of molecular super rotors . . . . 127

7 Outlook . . . . . . . . . . . . . . . . . . 133

7.1 Generation of $\mathrm{THz}$ radiation . . . . . . . . . . 133

7.2 Transient magnetization of paramagnetic gases . . . . . . . 133

7.3 Field-free alignment of asymmetric top molecules . . . . . . 134

7.4 Potential energy surface reconstruction . . . . . . . . . . 136

7.5 Harmonic rotational states . . . . . . . . . . . . . . . 136

7.6 Super rotors in He droplets . . . . . . . . . . . . . . 137

8 Conclusions . . . . . . . . . . . . . . . . . . . . 138

Bibliography . . . . . . . . . . . . . . . . . . 139 


\section{List of Tables}

3.1 Molecular constants used to fit the data in Fig. 3.6 . . . . . 58 


\section{List of Figures}

1.1 Illustration of (a) adiabatic vs. (b) non-adiabatic rotational excitation. . . . . . . . . . . . . 7

1.2 (a) Principle of an optical centrifuge excitation. A linearly polarized intense non-resonant laser field aligns the molecular axes along its polarization direction. This direction then undergoes accelerated rotation, sweeping the depicted red "corkscrew" shape and dragging the molecular axes along, eventually releasing them to freely rotate with a high angular frequency. (b) To produce the centrifuge field, one can combine two circularly polarized pulses of the opposite chirp and polarization handedness, shown in the figure. . . . . . . 9

1.3 Illustration of an adiabatic population transfer in the optical centrifuge. . . . . . . . . . . . . . 10 10

2.1 (a) Diagram of our femtosecond laser system. (b) Spectra of laser pulses before (red line) and after (blue line) amplification. 17

2.2 (a) Optical centrifuge shaper. IM: input mirror, G0, G1, G2: diffraction gratings, L1, L2, L3: $f=250 \mathrm{~mm}$ achromatic lenses, HM: half-cut mirror, FM: folding mirror, RR1, RR2: retroreflectors. Inset: movable shutter. (b) Laser spectra before (magenta), and after the shaping for the "blue" (blue) and "red" (red) arms. The "blue" arm spectrum is shown with the shutter completely out of (dashed), and half way in the beam (solid) . . . . . . . . . . . . . . 18

2.3 Combining the centrifuge arms. HWP: half-wave plate, M: mirror, PBS: polarizing beam splitter cube, QWP: quarterwave plate. . . . . . . . . . . . . . . 19

2.4 Far field profiles of the (a) "blue" arm, (b) "red" arm, (c)both beams combined. . . . . . . . . . . . 20

2.5 Illustration of the standard cross-correlation setup. . . . . . 20 
2.6 Time- and frequency-resolved characterization of the centrifuge beams. In each panel, the measurements related to the "blue" ("red") arm are colored blue (red). (a) Time-resolved cross correlation measurements. (b) Spectra of the beams. (c) Time-resolved spectrogram of each centrifuge arm. Tilted lines correspond to a frequency chirp. . . . . . . . . . 21

2.7 An illustration of Stokes (left) and anti-Stokes (right) rotational Raman scattering. . . . . . . . . . . . . . . . 22

2.8 An illustration of the rotational Doppler effect. Thick (thin) blue arrows show the circularly polarized probe field of the same (opposite) direction with respect to the molecular rotation (green arrows). . . . . . . . . . . . 23

2.9 Gas chamber for Raman spectroscopy. . . . . . . . . . . . 24

2.10 (a) Diagram of separate amplification of the two centrifuge beams. (b) Multi-pass amplifier. . . . . . . . . . . 25

2.11 (a) Probe beam setup. M: mirror, RR: retroreflector. (b) Full centrifuge spectrum (red), full probe spectrum (blue), and truncated probe spectrum (magenta) . . . . . . 26

2.12 Raman setup. PBS: polarizing beam splitter, QWP: quarterwave plate, DM: dichroic mirror, Filt: dichroic filter, L: lens, M: mirrors. . . . . . . . . . . . . . . 27 27

2.13 Raman spectrum of laser-kicked oxygen. . . . . . . . . . 28

2.14 REMPI principle. . . . . . . . . . . . . . . . . 29

2.15 Ultra-high vacuum chamber with a molecular source. . . . . . 30

2.16 (a) Magnified view of the UHV chamber. (b) Nozzle control electronics. (c) Gas line connections diagram. . . . . . . . . 31

2.17 Geometry of the time-of-flight spectrometer (left) and the simulated electric field distribution (right). . . . . . . .

2.18 (a) Raw time-of-flight spectrum of photo-dissociated mixture of gases. (b) Calibrated spectrum. (c) Magnified region of the spectrum around mass-to-charge ratio of $8 . \ldots 34$

2.19 Dye laser system. The flashlamps (FL) and Q-switch (Qsw) of Nd:YAG pump laser were synchronized with the centrifuge pulses electronically. The output of the dye laser was frequency doubled in a second harmonic generation system

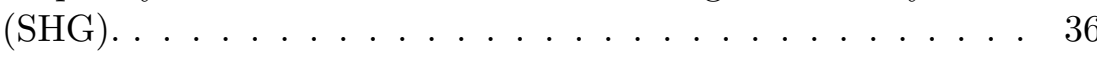


2.20 REMPI spectroscopy setup. Centrifuge beam is combined with a tunable UV laser pulse and focused inside a vacuum chamber on a supersonically expanded molecular jet between the charged plates of a time-of-flight (TOF) mass spectrometer. The ionization rate is measured with a multi-channel plate $(\mathrm{MCP}) . \ldots \ldots \ldots \ldots \ldots$

2.21 REMPI spectra of cold ( $T=10 \mathrm{~K}$, blue) and room temperature (red) nitrogen. Black ticks, connected by lines to guide the eye, show the calculated transition energies for different branches. . . . . . . . . . . . . . . .

2.22 REMPI spectra of cold ( $T=10 \mathrm{~K}$, blue) and room temperature (red) oxygen. Dashed line demonstrates the effect of power broadening. . . . . . . . . . . . .

2.23 Illustration of the $C^{3} \Pi_{g}\left(v^{\prime}=2\right) \leftarrow \leftarrow X^{3} \Sigma_{g}^{-}\left(v^{\prime \prime}=0\right)$ transition in oxygen. Blue (red) area marks the rotational structure of the ground (excited) electronic state, affected by the spinspin and spin-rotational (spin-orbit) interaction. . . . . . .

2.24 Illustration of the process of Coulomb explosion. After being ionized by a fs pulse, the molecules immediately fragment into ions recoiling in the direction of their respective internuclear axes. . . . . . . . . . . . . . . 41

2.25 Relevant polar variables for the velocity map imaging. . . . . 41

2.26 (a) Raw ion image after a single laser shot. (b) Processed image with the determined ion positions, marked by the magenta crosses. . . . . . . . . . . . . . .

$2.27 \mathrm{~N}^{+}$ion image distribution measured for 1,000,000 events, taken with probe polarization (a)parallel and (b)normal to the detector plane. . . . . . . . . . . . . . . 44 4

2.28 Definition of the centrifuge angles. . . . . . . . . 46

2.29 (a) Angle measurement setup. Ref: reference femtosecond laser pulse, BS: 50/50 beam splitter, L: lenses, Ret: glass plate retarder, PD1, PD2: photodiodes. (b) Correlation plot of the two normalized signals from PD1 and PD2, acquired over 100 laser shots. Red bar charts show the histograms of the two signals. Scatter plots of different colors correspond to three different delays between the reference and the centrifuge pulses, with the red trace corresponding to an optimal delay, used for determining $\theta_{\text {rel }} \ldots \ldots \ldots \ldots$. . . . . . . . 47 
3.1 Probe spectra after passing through the counter-clockwise (purple) and clockwise (cyan) centrifuged oxygen molecules. The appearance of only anti-Stokes or only Stokes sidebands indicate unidirectional rotation. . . . . . . . . . . . . 49

3.2 Time-dependent Raman shifts from the centrifuged $\mathrm{N}_{2}$ (a) and $\mathrm{H}_{2}$ (b) molecules. As the molecules spend longer time in the centrifuge, the observed Raman frequency shift increases along the slopped dashed lines, providing a direct evidence of accelerated molecular rotation. Some of the molecules "leak" from the accelerating angular trap, leaving the horizontal traces at the intermediate Raman shifts. Horizontal dashed lines correspond to the angular velocity of rotational states, mostly populated at room temperature. . . . . . . 50

3.3 State-resolved Raman spectra of centrifuged oxygen molecules. Higher curves correspond to longer spinning time inside the centrifuge. Red vertical arrows mark the rotational quantum numbers. . . . . . . . . . . . . . .

3.4 Experimental (dots with error bars) and calculated (dashed blue and solid green for the rigid and non-rigid rotor approximations, respectively) rotational energy spectrum expressed as a Raman frequency shift. . . . . . . . . . . . . . . 52

3.5 2D REMPI spectrogram for a linearly polarized probe. (a) Experimental spectra of cold (10 K, blue) and centrifuged molecules (yellow), along with a simulated spectrum of a "hot" thermal ensemble (3000 K, red) calculated with PGOPHER software [134]. (b) Ion signal as a function of the probe laser wavelength and molecular angular momentum defined by the centrifuge final rotation speed. Different areas of the $2 \mathrm{D}$ plot were measured with different sensitivities and probe intensities and are displayed with different color scales to compensate for the broad dynamic range of the data. c Vertical cross-sections of several consecutive peaks from one particular branch, shown in the inset to (b). The peaks are regularly separated with a distance of $\Delta N=2$ reflecting ${ }^{16} \mathrm{O}_{2}$ nuclear spin statistics. . . . . . . . . . . . . 554 
3.6 2D REMPI spectrogram for a circularly polarized probe. Electric field vector is counter-rotating (a) and co-rotating (b) with the centrifuged molecules. The directionality of laserinduced rotation results in the sensitivity of the measured signal to the handedness of probe polarization. The results of fitting the data to the theoretical model are shown with colored lines and markers for different branches and resonances, respectively. Branch nomenclature is the same as in 122. . . 56

3.7 Comparison of the observed REMPI data for the perturbed $F_{1}$ spin-orbit component with the calculations based on molecular constants from our work (red circles), White et al. [135] (blue triangles) and Lewis et al. 82] (purple squares) . . . . .

3.8 Ultra-high rotational resonances of $\mathrm{O}_{2}$. The two panels correspond to two possible ways of fitting the observed resonant branches (apparent along white dashed lines) to the calculated Hund's case (b) structure (labeled with markers). In panel (a), the upper branch corresponds to $\Delta N=-1$, and the lower one to $\Delta N=3$, resulting in $B_{v}=1.620 \mathrm{~cm}^{-1}$ and $D_{v}=4.4 \times 10^{-6} \mathrm{~cm}^{-1}$. In panel (b), the upper branch overlaps with $\Delta N=-2$, whereas the lower one with $\Delta N=2$, yielding $B_{v}=1.664 \mathrm{~cm}^{-1}$ and $D_{v}=5.7 \times 10^{-6} \mathrm{~cm}^{-1}$. . . 59

3.9 Observed linewidths of $J^{\prime}=N^{\prime}-1$ (triangles) and $J^{\prime}=$ $N^{\prime}$ (squares) spin-orbit sublevels of $C^{3} \Pi_{g}\left(v^{\prime}=2\right)$ level as functions of rotational quantum number $N^{\prime}$. Inset demonstrates a fit of experimental data (solid red) to a sum of lorentzians in order to extract linewidths. Absolute position, absolute area and the widths of two peaks were fitted for each doublet individually, with the areas ratio fixed to a value extracted from the best resolved $N^{\prime}=115$ doublet and with a doublet line separation equal to a calculated one. . . . . . 60 60

3.10 (a) Linear rotor angular distributions. Experimental VMI images of $\mathrm{N}_{2}$ fragments with the centrifuge turned off (b) and on $(\mathbf{c}) \ldots \ldots \ldots \ldots \ldots$ 
4.1 (a) Selective centrifuge spinning of oxygen to $N \approx 109$. Dashed tilted line shows the increasing angular frequency of the centrifuge, terminated at about 90 ps. Dashed horizontal line marks the most populated rotational state of $\mathrm{O}_{2}$ at room temperature, $N=7$. Oscillations of the coherent rotational wave packet are shown in the inset. (b) Truncating the probe spectrum to $0.1 \mathrm{~nm}$ allowed us to resolve individual rotational Raman transitions, similar to Fig. 3.2 , at the expense of losing time resolution. . . . . . . . . . . . . 64

4.2 Time evolution of a wide (top) and a narrow (bottom) rotational wave packet, consisting of many or only two rotational states, respectively. The latter is an example of a quantum "cogwheel state". $T_{\text {rev }}$ is the rotational revival time. . . . . 65

4.3 Experimental VMI of $\mathrm{O}_{2}$ taken with the centrifuge turned off (a) and on (b). The apparent alignment of the cold ensemble in the plane of probe polarization $(\mathrm{PP})$ in panel (a) is due to the geometric alignment effect. The enhancement of this alignment in panel (b) is caused by the centrifuge. (c) Geometry of the in-plane distribution measurements. . . . . .

4.4 Normalized population of the rotational states with $N=37$ (blue) and $N=39$ (red) as a function of the centrifuge final angular velocity. The black arrow shows the terminal angular frequency of the centrifuge used in this work for creating rotational wave packets in $\mathrm{O}_{2} \ldots \ldots \ldots \ldots$. . . . . .

4.5 (a) Probability density as a function of the molecular angle and the free propagation time. Time zero corresponds to approximately 100 ps since the release from the centrifuge. The blue dashed line marks the calculated trajectory of a "dumbbell" distribution rotating with the terminal angular frequency of the centrifuge, whose classical period is indicated with the white horizontal bar at the lower right corner. Zoomin to the region near (b) $\frac{3}{8} T_{\text {rev }}$ and (c) $\frac{3}{8} T_{\text {rev }}$, taken with better averaging and angular resolution. Twice higher density of the tilted lines in panel (c) (4 per classical period indicated by the tilted arrows) stems from the emergence of a "cross"shaped distribution with four lobes along two perpendicular directions. . . . . . . . . . . . . . . 
4.6 Probability density as a function of the molecular angle and the the free propagation time of $\mathrm{D}_{2}$ prepared in the equalweight superposition of $N=2$ and $N=4$ states. The observed nondispersing behavior illustrates the main property of a quantum cogwheel state. The classical period is indicated with the white horizontal bar at the lower right corner. . . .

4.7 (a): Spin-rotational splitting of two rotational levels of oxygen, $N^{\prime \prime}$ and $N^{\prime}=N^{\prime \prime}+2$. Each level is split into three sub-levels with energies $F_{k}, k=1,2,3$ for the total angular momentum $J=N+1, N, N-1$, respectively. Three strongest Raman transitions (out of the total six allowed by the selection rules) corresponding to the $S\left(N^{\prime}\right)$ branch are shown and labeled according to the participating $J$-states. (b): Dependence of the three Raman frequencies ( $\Omega_{k}$ for $S_{k}$ line) on the rotational quantum number. . . . . . . . . . . . 74

4.8 (a): Experimentally detected Raman spectrogram of centrifuged oxygen showing the rotational Raman spectrum as a function of the time delay between the beginning of the centrifuge pulse and the arrival of the probe pulse. Color coding is used to reflect the signal strength in logarithmic scale. (b): Cross-section of the two-dimensional spectrogram at the delay of 200 ps (vertical dashed line in a), showing an ultra-broad rotational wave packet created by the optical centrifuge. (c): Spin-rotation oscillations of the $N=91$ Raman line (horizontal dashed line in a). Experimental uncertainty is indicated by the vertical error bars. Note logarithmic scale in all panels. 76

4.9 The observed data (blue circles, normalized to 1 at $t=100 \mathrm{ps}$ ) and the fit to spin-rotation oscillations (red curves, Eq 4.5) for six different Raman lines corresponding to the rotational quantum numbers $N=5,7,9,21,61$ and 101. Experimental uncertainty (not shown) is similar to Fig. 4.8 (c). Note logarithmic scale in all panels. . . . . . . . . . . 77 
4.10 (a) Experimental setup for detecting the plane of molecular rotation. The molecules are excited with a centrifuge pulse, then rotate freely in a magnetic field of a permanent magnet, and finally are ionized with a femtosecond probe pulse. The resulting atomic ions are extracted and focused with ion optics of a velocity map imaging (VMI) apparatus onto a microchannel plate detector (MCP) with a phosphor screen. The images observed without (b) and with (c) the rotational excitation by a centrifuge pulse. . . . . . . . . 80

4.11 Ion images of oxygen super rotors evolving in the external magnetic field. Different rows correspond to different degrees of rotational excitation ( $N$ value on the left), whereas each column corresponds to the evolution time indicated at the bottom. The initial disk distribution splits into three disks precessing with different frequencies according to their spin

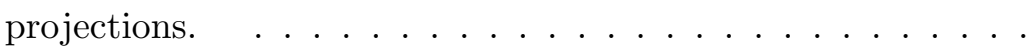

4.12 Experimentally determined precession period as a function of the rotational quantum number (blue squares) and the expected theoretical dependence (dashed red line). . . . . . . .

4.13 (a) Ion images of nitrogen super rotors in the external magnetic field. To improve the visibility of precession, an oversaturated central region is cut off and contour lines (green dashed) are added. (b) Directional REMPI signal as a function of the pump-probe delay. Maximum (minimum) signal is detected when the molecules rotate in the opposite (same) direction with respect to the circularly polarized probe. Solid red and dashed blue lines correspond to the opposite initial directions of molecular rotation, i.e. opposite handedness of the centrifuge pulse. . . . . . . . . . . . .

5.1 (a) Illustration of the concept of a "two-dimensional (2D) centrifuge". The three-dimensional corkscrew-shaped surface represents the field of a conventional "3D centrifuge", propagating from right to left. Shown in blue is the field of a $2 \mathrm{D}$ centrifuge, created by passing the 3D centrifuge through a linear polarizer. (b, c) Simulated molecular distribution after the rotational excitation by a $2 \mathrm{D}(\mathbf{b})$ and a $3 \mathrm{D}(\mathbf{c})$ optical centrifuge, propagating along the $\hat{x}$-axis. . . . . . . . 
5.2 (a) Rotational Raman spectra of the centrifuged oxygen molecules recorded with a linearly polarized probe light. From top to bottom, the spectra correspond to the excitation by a single femtosecond pulse (black), a "truncated" 2D centrifuge (green), a full 2D centrifuge (red), and a full 3D centrifuge (blue) with a terminal rotational frequency around $10 \mathrm{THz}$. The spectra have been recorded at the delay times of 400 ps. (b) Rotational Raman spectra of the centrifuged oxygen molecules recorded with a circularly polarized probe light. Upper red and lower blue curves correspond to the 2D and $3 \mathrm{D}$ centrifuge, respectively. The spectra have been recorded at the delay times of $400 \mathrm{ps} \ldots \ldots \ldots \ldots$

5.3 Ion images of nitrogen molecules prior to any rotational excitation (a), and following the excitation by a $3 \mathrm{D}(\mathbf{b})$ and a $2 \mathrm{D}$ (c) centrifuge. Calculated molecular distributions are shown above the respective images. . . . . . . . . . . . .

5.4 (a) Definition of the spherical coordinates $(r, \theta, \phi)$ and polar coordinates $(R, \Theta)$, used to describe the full molecular distribution and its two-dimensional projection on the $x z$ plane of the ion detector. (b) $R$-dependence of the extracted alignment factor, with the shaded region indicating the experimental error. (c) Ion image of $\mathrm{N}_{2}$ molecules aligned with a $2 \mathrm{D}$ optical centrifuge. The black dotted and white dashed lines show the circular cross-sections at the radius of a maximum ion signal, $R=R_{\max }$, and the bigger radius used for estimating the true two-dimensional alignment factor, respectively. The latter is also marked by the red dashed line in plot (b). .

5.5 (a) Setup for the detection of the "magneto-rotational" birefringence. BS: beam splitter, DM: dichroic mirror, LP (LA): linear polarizer (analyzer) oriented at angle $\theta_{p}\left(\theta_{p}+90^{\circ}\right)$ with respect to $\vec{B}$, DL: delay line, L: lens, M: two magnetic coils connected in a Helmholtz configuration. ' $\mathrm{O}_{2}$ ' marks the pressure chamber filled with oxygen gas under pressure $P$ at room temperature. An optical centrifuge field is illustrated above the centrifuge shaper with $\vec{k}$ being the propagation direction and $\vec{E}$ the vector of linear polarization undergoing an accelerated rotation. (b) Geometry of the magnetic and optical fields used in this work. The cloud of centrifuged molecules is depicted as a dark ellipse. . . . . . . . . . . . . 94 94 
5.6 Spectrum of probe pulses transmitted through the ensemble of centrifuged oxygen molecules as a function of the applied magnetic field. All spectra have been recorded at the probe delay of $t=1.14 \mathrm{~ns}$ and under $0.3 \mathrm{~atm}$ of gas pressure. Crossed circular (rather than linear) polarizer and analyzer were used here to detect a weak magneto-rotational Raman signal (note the change of vertical scale $(\times 200)$ at frequencies higher than $7 \mathrm{THz}) . \ldots \ldots \ldots \ldots$

$5.7(\mathbf{a}, \mathbf{b})$ Calculated angular distribution of the molecular axes for the rotational state with $N=59$ at time $t=0.9 \mathrm{~ns}$ in an external magnetic field of 0 and 0.32 Tesla, respectively. (c) Birefringence signal (scaled to peak at 1) as a function of angle $\theta_{p}$ between the polarization of probe pulses and the magnetic field direction. Black circles: data taken at $2 \mathrm{~T}$, $t=1.5 \mathrm{~ns}$, and $N=95$. Red curve is a fit to $\cos ^{2}\left(\theta_{p}\right)$. (d) Experimentally measured distribution of molecular axes, imaged in the direction of the applied field. All parameters are the same as in panel $(\mathbf{b}) . \ldots \ldots \ldots \ldots$

5.8 Decay of the birefringence signal for different values of the rotational quantum number at $B=2 \mathrm{~T}$. All solid curves are generated by spline-fitting the experimental data (shown with colored markers) and normalized to peak at 1 . The corresponding lifetimes are $85 \pm 10,290 \pm 20,660 \pm 50$ and $610 \pm 50 \mathrm{ps} \cdot \mathrm{atm}$ for $N=13,33,73$ and 99 , respectively. . . . 98

5.9 Dependence of the birefringence signal recorded at $t=1 \mathrm{~ns}$ on the strength of the applied magnetic field. . . . . . . . . . . 99

5.10 Illustration of the main concept of aligning an asymmetric top molecule $\left(\mathrm{SO}_{2}\right)$ with an optical centrifuge. Left side of the centrifuge pulse (red corkscrew surface) corresponds to its leading edge, linearly polarized along $\vec{E}$. Behind the trailing edge of the centrifuge (right side), the molecular plane is aligned in the plane of the induced rotation. The three axes of $\mathrm{SO}_{2}(a, b$ and $c)$ are shown in red, green and blue, respectively. $\theta_{b}$ is the orientation angle measured in this study.100

$5.11(\mathbf{a}, \mathbf{b})$ Images of $S^{3+}$ and $S^{2+}$ fragments originated from the rotationally cold and centrifuged $\mathrm{SO}_{2}$ molecules, respectively.(c) Setup for the in-plane distribution measurement of the molecular $b$-axis (green dashed line). PP marks the polarization plane of the lasers. . . . . . . . . . . . . . . 101 
5.12 Illustration of the effect of underestimated and overestimated alignment of $\mathrm{S}^{3+}$ and $\mathrm{SO}^{2+}$, respectively. . . . . . . . . 102

5.13 (a) Periodic revivals of the calculated two-dimensional alignment factor $\beta_{2 D}=\left\langle\cos ^{2} \theta\right\rangle_{2 D}-\frac{1}{2}$, determined from (b) Time evolution of the molecular in-plane angular distribution. (c) High resolution time scan around the alignment peak. . . . . 104

5.14 Schematic rotational spectrum of asymmetric top molecules. . 105

5.15 Revival period as a function of the rotational frequency of $\mathrm{SO}_{2}$, with the rotational quantum numbers shown along the upper horizontal axis. Experimental data (blue circles) are compared with the results of classical calculations, in which the potential energy surface is expanded to second and fourth order in deformation coordinates (dashed and solid lines, respectively). The inset shows the dependence of the $2 \mathrm{D}$ alignment $\beta_{2 D}$ on the pump-probe delay, used for calculating the revival time at two angular frequencies, $18.8 \mathrm{rad} / \mathrm{ps}$ (magenta) and $39.3 \mathrm{rad} / \mathrm{ps}$ (green), labeled with the correspondingly colored stars in the main plot. Solid lines represent experimental data, while the dashed lines show the fitting functions used to extract the revival period. . . . . . . . . 106

6.1 Experimentally detected Raman spectrogram of nitrogen showing the rotational Raman spectrum as a function of the time delay between the beginning of the centrifuge pulse and the arrival of the probe pulse. Color coding is used to reflect the signal strength. Tilted white dashed line marks the linearly increasing Raman shift due to the accelerated rotation of molecules inside the 100 ps long centrifuge pulse. A onedimensional cross section corresponding to the Raman spectrum at $t=270 \mathrm{ps}$ is shown in white. . . . . . . 111

6.2 Logarithm of the intensity of the experimentally measured Raman lines as a function of time (normalized to 1 at $t=200$ ps, chosen so as to avoid the effects of the detector saturation at earlier times). Dots of the same color represent experimental data for one particular value of the rotational quantum number. Black solid lines show the numerical fit to the corresponding exponential decay. Data collected at $P=0.75 \mathrm{~atm}$ and $T=294$ K. . . . . . . . . . . . . . . . . 112 
6.3 Decay rate of rotational coherence of $\mathrm{N}_{2}$ as a function of the rotational quantum number at two different temperature values, $T=294 \mathrm{~K}$ (blue triangles) and $T=503 \mathrm{~K}$ (red circles), expressed as the Raman linewidth. Solid curves correspond to the prediction of the simplified "energy corrected sudden" (ECS) approximation at $N>12$. Black squares represent the

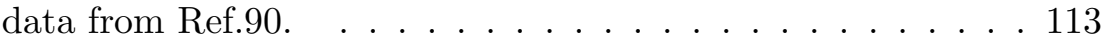

6.4 The dependence of the adiabaticity factor $a$ on $N$ for the nitrogen gas temperatures of $294 \mathrm{~K}$ (blue line), $503 \mathrm{~K}$ (red line) and $1100 \mathrm{~K}$ (green line). Solid horizontal line marks the adiabaticity threshold, $a=\pi$. Black filled circles mark the rotational evels accessed in previous experiments on rotational decoherence. . . . . . . . . . . . . . . . 115

6.5 The decay rate of rotational coherence in oxygen (blue circles) and nitrogen (green triangles) as a function of the frequency of molecular rotation. For convenience, rotational quantum numbers of $\mathrm{O}_{2}$ and $\mathrm{N}_{2}$ are shown below the frequency axis, and the decay times $\tau_{N}$ are shown on the right vertical axis. Black asterisks and grey squares depict the data from [92] and [90], respectively, where the rotational decay has been studied in thermal oxygen by two different techniques. Solid red (dashed black) curve shows the result of fitting the adiabaticity correction factor $\Omega_{l_{c}}(N)$ to the data for oxygen (nitrogen) as discussed in text. . . . . . . . . . . . 116

6.6 (a) Schematic diagram of the imaging setup. Nanosecond or picosecond probe pulses (green and blue beams, respectively) propagated either collinear with, or perpendicular to the centrifuge, creating an image of the rotationally excited volume of gas on a CCD camera either in the longitudinal or transverse direction, respectively. DM: dichroic mirror, FL: focusing lens, IL: imaging lens, KE: knife edge (shown along $y$ instead of $z$ axis for clarity), LP: linear polarizers at $\pm 45^{\circ}$ to $y$ axis, F: frequency filter. An example of the longitudinal image with a circularly expanding sound wave is shown in panel (b). Images in the transverse geometry were taken at early (c), intermediate (d) and late (e) time moments. Image (c) was recorded with the two linear crossed polarizers in place. Schlieren image (e) was recorded with the knife edge in place. . . . . . . . . . . . . . . . . . . . 118 
6.7 Radial cross-section of the images, representing the change in the refractive index of the gas, taken along the centrifuge beam, as a function of time, recorded on a long timescale. . . 120

6.8 (a) Radial cross-section of the images, taken along the centrifuge beam, as a function of time, recorded on a short timescale. The arrow at around 7 ns shows the crossover between the rotation-induced and thermal channels. Sample images of both refractive channels are shown in the square insets. (b) Image contrast as a function of time. The red line on top of the experimental data points is shown to guide the eye. . . . . . . . . . . . . . . . . . 121

6.9 Radial cross-section of the images, taken along the centrifuge beam, as a function of time recorded in the gas of $\mathrm{N}_{2} \mathrm{O}$ molecules at the pressure of $0.5 \mathrm{~atm}$ (red line) and $0.25 \mathrm{~atm}$ (blue line). Shaded regions around each line represent the statistical error (one standard deviation) in our experimental data. . . . . . . 122

6.10 Birefringence signal (red triangles) and image contrast (blue circles) of the centrifuged gas, retrieved from the transverse images taken with and without the crossed polarizers (left and right insets, respectively) as a function of time. Two data points in the lower right corner (within the dashed rectangle) indicate the drop in the phase contrast for the slower rotating centrifuge. The solid black line is a fit to an exponential decay of the birefringence signal, which gave $\tau_{b}=3.4 \mathrm{~ns}$ as the decay time. This time constant was used in the hydrodynamic numerical calculations of $\Delta n(\tau)$, shown by the dashed black line. The dashed magenta and green lines correspond to the same calculations, performed with the decay constant being five times shorter and longer, respectively. The horizontal dash-dotted line indicates the noise floor for the phase contrast measurement. . . . . . . . . . . . . . 123 
6.11 Comparison of the experimental results with the numerical calculations of gas hydrodynamics. The calculated change in the gas density, $\Delta \rho$, is shown in panel (a) as a function of time and distance. Experimentally determined parameters of the observed gyroscopic channel are used to simulate the heat source, which initiates the dynamics at point $(y=0, \tau=0)$. In panel (b), the derivative of the calculated density profile $\mathrm{d} / \mathrm{d} y[\rho(y)]$ at $1.2 \mu \mathrm{s}$ is compared with the $y$ cross-section of the schlieren image recorded $1.2 \mu \mathrm{s}$ after the centrifuge (dashed red and solid blue curves, respectively). The dependence of the measured and calculated schlieren signals on both space and time is shown in panels $(\mathbf{c})$ and $(\mathbf{d})$, respectively, with the two lines indicating the two cross-sections displayed in plot (b). . . . . . . . . . . . . . . . . 125

6.12 Comparison of the birefringence decay for the 2D centrifuge with a full spectral bandwidth of $20 \mathrm{THz}$ (red diamonds) and with its bandwidth truncated at $\approx 10 \mathrm{THz}$ (purple triangles) and $\lesssim 5 \mathrm{THz}$ (green squares). Black circles represent the case of a 1D kick. In all plots, solid lines show the fits by exponential decays. . . . . . . . . . . . . . . 126

6.13 Typical rotational Raman spectrum of oxygen super rotors measured with a probe pulse delayed by 200 ps with respect to the centrifuge pulse. Inset: an example of the acoustic signal recorded in centrifuged ambient air. . . . . . . . . . 128

6.14 Amplitude of the recorded sound as a function of the energy of centrifuge pulses, plotted on a log-log scale. Each data set consists of 10,000 points. (a) Typical acoustic signal from the centrifuged gas of nitrogen molecules (blue diamonds) is compared to the sound generated by the centrifuge in pure argon at the same pressure of $95 \mathrm{kPa}$ (green triangles). (b) Acoustic response of the centrifuged oxygen with and without a small admixture of $\mathrm{SF}_{6}$ molecules (black dots and red circles, respectively). Black dashed lines in both panels show fits to power-law scaling. . . . . . . . . . . . . . . . . 129 
6.15 Amplitude of the recorded sound as a function of the rotational energy deposited in the gas sample. (a) Acoustic response from the centrifuged gas of nitrogen molecules (blue circles) is compared to the sound generated by the centrifuge in pure argon at the same pressure of $95 \mathrm{kPa}$ (green triangles). (b) Acoustic response from the centrifuged oxygen with and without a small admixture of $\mathrm{SF}_{6}$ molecules (black dots and red circles, respectively). All insets show Raman spectra corresponding to the data points marked with black crosses and plotted as a function of rotational frequency in THz. . . . . . 130

7.1 Precession of the angular distributions (blue disks) of paramagnetic spin-1 super rotors in magnetic field. Different rows correspond to different spin projections $S_{N}$. Blue and green arrows represent the angular momentum and spin vectors, respectively. . . . . . . . . . . . . . . . 134

7.2 Calculated distributions of molecular axes $a$ (red), $b$ (green) and $c$ (blue) of an asymmetric top molecule in the aligned rotational state. . . . . . . . . . . . . . . 135 


\section{Acknowledgements}

I would like to express my sincere gratitude to my supervisor Valery Milner for giving me the opportunity to work in a unique creative atmosphere of the Ultrafast Coherent Control Group. This work would not be possible without his thoughtful guidance and his scientific vision.

I am very grateful to Alexander Milner for sharing his vast knowledge of experimental physics and life. Being always open to discussions about anything, from the laws of nature to Strugatsky's books, he made everyday lab routine a truly enjoyable experience.

I would like to thank our collaborators John Hepburn, Ilya Averbukh and Johannes Floss for the fruitful discussions, which inspired many interesting ideas.

I am very thankful to Gilad Hurvitz, Sergey Zhdanovich and Guillaume Bussiere for their help and valuable advice on the experimental setup, and to the rest of the Ultrafast Coherent Control Group, especially Alice Schmidt-May, Martin Bitter, Koosha Rezaiezadeh, Oleksandr Litvinov, Ian MacPhail-Bartley and Alexander Ruf for their contribution to the friendly and creative research environment.

Finally, I am very grateful to my wife Natasha for her support and encouragement of my academic pursuits and her patience during my latenight shifts in the lab and to my parents Olga and Victor for first inspiring the interest in science in me. 


\section{Chapter 1}

\section{Introduction}

Control of molecular rotation has been long recognized and successfully used as a powerful tool for controlling a broad range of physical processes. In the most popular and developed scenario, such control is achieved by spatial alignment or orientation of the axes of gas-phase molecules in the laboratory frame. Here, the direction of molecular axes is set by either a line (alignment), or a vector (orientation). First works on this subject date back to 1960-s, when the groups of Brooks and co-workers and Beuhler and coworkers crossed a beam of methyl iodide $\mathrm{CH}_{3} \mathrm{I}$ with a beam of alkali ( $\mathrm{K}$ or $\mathrm{Rb}$ ) atoms. $\mathrm{CH}_{3} \mathrm{I}$ molecules were oriented by means of a hexapole rotational filtering followed by applying a homogeneous electric field. The researchers observed a substantial increase in the yield of KI or RbI when the alkali atom was approaching $\mathrm{CH}_{3} \mathrm{I}$ from the side of the I atom, compared to when it was approaching from the opposite side $[9,10,87,95]$. Later, Loesch and Stienkemeier [84, 85] showed, that similar control of gas-phase reaction, can be achieved by alignment, rather than orientation. Observing the reactions $\mathrm{Sr}+\mathrm{HF} \rightarrow \mathrm{SrF}+\mathrm{H}, \mathrm{K}+\mathrm{HF} \rightarrow \mathrm{KF}+\mathrm{H}$, they demonstrated control of the reaction yield, by changing the $\mathrm{HF}$ alignment angle relative to the approach direction. Similarly to gas phase, molecular orientation was used for controlling gas collisions with a solid surface. Kuipers et al. 73 . used a beam of cold oriented NO and showed that the rotational excitation acquired by the molecules scattered off a solid flat silver surface, dramatically depended on whether the molecule was approaching the metal with its O- or N-end. Shreenivas et al. 116] proposed and theoretically calculated the possibility of controlling the orientation of the absorbed iodine on silicon surface by aligning $\mathrm{I}_{2}$ molecules prior to collision.

The works mentioned above utilized alignment and orientation to modify the way molecules interact with matter. A different possibility is to control their interaction with an external electromagnetic field. Itatani and coworkers used the molecular alignment techniques to pin $\mathrm{O}_{2}$ and $\mathrm{N}_{2}$ to the laboratory frame [53, 54. In a tunnel ionization process induced by a strong laser field, an electron was removed from the molecular orbital and recollided with it, emitting extreme ultraviolet radiation, whose intensity 
depended on the angle between the molecular axis and the electron ejection direction. This information was used by the authors to extract the properties of the molecular orbitals. The dipole force, produced by the interaction of inhomogeneous laser field with the dipole moment it induced in a particle, became an important tool for controlling the spatial position of the particles. Purcell et al. showed [101], that the alignment can be an effective tool for controlling molecular polarizability due to its high anisotropy and, therefore, the dipole force.

In 1999, Karczmarek et al. proposed a novel tool, employing an intense non-resonant laser pulse tailored in a special way, to spin diatomic molecules to quantum states of extremely high angular momentum [57]. The molecular axes were first aligned along the pulse polarization and then followed its accelerated rotation. The shaped laser pulses were dubbed "an optical centrifuge". The technique was later demonstrated to spin $\mathrm{Cl}_{2}$ molecules so fast, that their bonds couldn't withstand the centrifugal forces, causing the molecule to dissociate [130]. At the same time, Li et al. proposed their own technique of producing similar highly rotationally excited diatomic molecules of $\mathrm{Li}_{2}[83]$. Although their method was never experimentally realized, they predicted that these molecules, which they called "super rotors", will exhibit unique collisional properties. Since then, several theoretical works were published, suggesting a number of interesting applications of super rotors.

Hasbani and coworkers proposed to use an optical centrifuge to selectively break molecular bonds [43. Similar to the original work by Villeneuve et al. [130], the idea was to spin HCN molecules up to a dissociation. Due to a much larger centrifugal pull from the heavy $\mathrm{N}$ atom, compared to the light $\mathrm{H}$, it was predicted that the much stronger $\mathrm{C}-\mathrm{N}$ bond will break first. Gershnabel et al. studied the effect of molecular alignment and rotation on the dipole force from a focused laser beam and suggested an optical centrifuge to be a promising tool for creating rotational states, strongly antialigned to a plane of rotation [39]. Numerical simulations of Khodorkovsky and coworkers suggested a method of controlling the scattering direction of super rotors from a solid surface [61]. Steinitz et al. considered a dense gas of rotationally excited molecules [120]. As the rotational energy dissipated and the molecules reached thermal equilibrium, their high internal angular momentum was transforming to a macroscopic angular momentum of gas motion, causing a vortex to form. Calculations of Hartman et al. [42] showed the increasing stability of molecular super rotors towards the collisions with a thermal ensemble, whereas Forrey 33 ] has proposed to use this stability for sympathetic cooling of molecules. 


\subsection{Rotational motion of molecules}

Consider a molecule, consisting of two atoms A and B. Its Hamiltonian is given by

$$
\hat{H}=-\frac{\hbar^{2}}{2 M_{A}} \nabla_{\mathbf{r}_{A}}^{2}-\frac{\hbar^{2}}{2 M_{B}} \nabla_{\mathbf{r}_{B}}^{2}+U\left(\mathbf{r}_{B}-\mathbf{r}_{A}\right),
$$

where $\hbar$ is the reduced Planck's constant, $M_{A, B}$ are the masses of atoms A and $\mathrm{B}$, respectively, Laplacians $\nabla_{\mathbf{r}_{A, B}}^{2}$ are taken with respect to the radius vectors $\mathbf{r}_{A, B}$ of atoms $\mathrm{A}$ and $\mathrm{B}$, respectively and $U\left(\mathbf{r}_{B}-\mathbf{r}_{A}\right)$ is the potential energy of interaction between the atoms. Introducing the new vectors $\mathbf{r}=$ $\frac{M_{A} \mathbf{r}_{A}+M_{B} \mathbf{r}_{B}}{M_{A}+M_{B}}$ and $\mathbf{R}=\mathbf{r}_{A}-\mathbf{r}_{B}$, we can rewrite (1.1) as a sum:

$$
\hat{H}=-\frac{\hbar^{2}}{2\left(M_{A}+M_{B}\right)} \nabla_{\mathbf{r}}^{2}-\frac{\hbar^{2}}{2 \mu} \nabla_{\mathbf{R}}^{2}+U(\mathbf{R}),
$$

where $\mu=\frac{M_{A} M_{B}}{M_{A}+M_{B}}$ is a reduced mass of the molecule. The first term corresponds to the kinetic energy of motion of the molecule as a whole; it disappears in the frame of reference, moving with the molecular centre of mass. When the molecule is not rotating too fast and the arising centrifugal forces are too weak to significantly stretch its bond, it is useful to introduce the so-called rigid rotor approximation. In this approximation, the interatomic distance $R=|\mathbf{R}|$ is assumed constant, leaving only the second term in (1.2). Moreover, since the wave function now depends only on the polar and azimuthal angles of $\mathbf{R}$ ( $\theta$ and $\phi$, respectively), the Laplacian term $-\frac{\hbar^{2}}{2 \mu} \nabla_{\mathbf{R}}^{2}$ simplifies to:

$$
\begin{array}{r}
\hat{H}=-\frac{\hbar^{2}}{2 \mu R^{2}}\left(\frac{1}{\sin \theta} \frac{\partial}{\partial \theta}\left(\sin \theta \frac{\partial}{\partial \theta}\right)+\frac{1}{\sin ^{2} \theta} \frac{\partial^{2}}{\partial \phi^{2}}\right)= \\
-\frac{\hbar^{2}}{2 I}\left(\frac{1}{\sin \theta} \frac{\partial}{\partial \theta}\left(\sin \theta \frac{\partial}{\partial \theta}\right)+\frac{1}{\sin ^{2} \theta} \frac{\partial^{2}}{\partial \phi^{2}}\right)
\end{array}
$$

where $I=\mu R^{2}$ is the molecular moment of inertia.

The eigenfunctions of this Hamiltonian, spherical harmonics $Y_{J}^{M_{J}}(\theta, \phi)$, are parametrized by the two quantum numbers, $J=0,1,2, \ldots$ and $M_{J}=$ $-J,-J+1, \ldots, J$, called the total angular momentum quantum number and the magnetic quantum number. These numbers have a meaning of the value of the total angular momentum and its projection onto a polar axis $z$, expressed in units of $\hbar^{1}$. The eigenenergies, corresponding to these states

\footnotetext{
${ }^{1}$ The exact value of the angular momentum is rather $\hbar \sqrt{J(J+1)}$, which becomes close to $\hbar J$ at large $J$.
} 
are given by

$$
\epsilon_{J}=\frac{\hbar^{2}}{2 I} J(J+1)=B J(J+1),
$$

where we introduced constant $B$, called the rotational constant. The energy, as expected, depends quadratically on the angular momentum. Since it doesn't depend on $M_{J}$, each $J$-state is $(2 J+1)$-fold degenerate over the possible values of $M_{J}$. Because the molecular bond may stretch, fast rotation would cause an increase of the moment of inertia, introducing higher order terms in the so-called Dunham expansion:

$$
\epsilon_{J}=B J(J+1)+D J^{2}(J+1)^{2}+\ldots
$$

Rotational spectrum of real diatomic molecules becomes more complicated for the states with a non-zero electron angular momentum. The total angular momentum $J$ is, as before, a conserved quantity, but now it is a sum of the angular momenta of the nuclear and electronic motion. For example, diatomic oxygen $\mathrm{O}_{2}$, frequently used in our experiments, has an electron spin of $S=1$ in its ground electronic state. Due to the zero electron orbital momentum it follows Hund's case (b) coupling, and its rotational energy does not follow Eq.(1.5) anymore [11]. However, if we introduce the angular momentum excluding electron spin $\mathbf{N}=\mathbf{J}-\mathbf{S}$, which in the case of oxygen corresponds to the rotation of its nuclei, the spectrum will still be following Eq.(1.5) with $J$ substituted by $N$. Each $N$-state is split into three closely spaced spin substates due to the spin-spin and spin-rotational interaction. For that reason $N$, rather then $J$, is often used below as a quantum number designating the degree of rotational excitation in oxygen. Sometimes, for consistency, it is also used for other molecular species, such as $\mathrm{N}_{2}$, with zero electron spin, making $N$ and $J$ equivalent.

\subsection{Control of rotational states with intense non-resonant laser fields}

The appeal of rotational control has stimulated the development of multiple techniques utilizing external electromagnetic fields [37, 119]. The most prominent class of such techniques involves subjecting molecules to strong non-resonant laser pulses [36, 119] in the visible or near-infrared region.

The interaction between the non-resonant laser field and the molecules could be described as follows. Consider a molecule placed in an oscillating electric field. The characteristic timescale of nuclear motion typically 


\subsection{Control of rotational states with intense non-resonant laser fields}

spans from 100 femtoseconds up to tens and hundreds of picoseconds, much longer than the period of laser light of a few femtoseconds. This means that the heavy nuclei are essentially frozen on the time scale of the field oscillations. On the contrary, the light electrons are typically moving on a shorter time scale and would adiabatically adapt to the electric field, stretching the molecular orbital in the direction of the field vector and producing an induced dipole moment. The expectation value of this dipole moment over the electronic state, in the first order with respect to the electric field $\mathbf{E}(t)$, could be expressed as

$$
\mu_{\rho}^{\mathrm{ind}}=\sum_{\rho^{\prime}} \alpha_{\rho \rho^{\prime}}^{\mathrm{LF}}\left(\mathbf{r}_{1}, \mathbf{r}_{2}, \ldots, \mathbf{r}_{\mathbf{N}}\right) E_{\rho^{\prime}}(t)
$$

where the molecular polarizability tensor $\alpha_{\rho \rho^{\prime}}^{\mathrm{LF}}$ describes the adiabatic electronic response to the field and parametrically depends on the positions of $N$ nuclei $\mathbf{r}_{\mathbf{1}}, \mathbf{r}_{\mathbf{2}}, \ldots, \mathbf{r}_{\mathbf{N}}$ and indices $\rho, \rho^{\prime}$ go over Cartesian laboratory-fixed coordinates $x, y, z$. In the rigid rotor approximation the positions of the nuclei can be fully described by the three Euler angles $(\theta, \varphi, \chi)$, characterizing the orientation of molecule in a laboratory-fixed frame. The potential energy of the electric field-induced dipole interaction can then be written as:

$$
V_{\text {int }}(t)=-\frac{1}{2} \boldsymbol{\mu}_{\text {ind }}(t) \cdot \mathbf{E}(t)=-\frac{1}{2} \sum_{\rho \rho^{\prime}} \alpha_{\rho \rho^{\prime}}^{\mathrm{LF}}(\theta, \varphi, \chi) E_{\rho}(t) E_{\rho^{\prime}}(t),
$$

where the factor of $1 / 2$ accounts for the induced nature of the dipole. Due to the time scale separability, mentioned above, it is natural to break the time dependence of the laser field into a slowly varying envelope and fast oscillating components,

$$
\mathbf{E}(t)=\frac{1}{2}\left(\varepsilon(t) e^{i \omega t}+\text { c.c. }\right) .
$$

In the equation above, $\varepsilon(t)=\hat{\varepsilon} \varepsilon(t)$, with $\hat{\varepsilon}$ and $\varepsilon(t)$ being the complex unit polarization vector and slowly varying pulse envelope, and $\omega$ is the angular frequency of light. For the purpose of studying rotational dynamics, we can now average the interaction potential (1.6) over the irrelevantly fast oscillations of the field in Eq.(1.7) to get the effective interaction potential 
for the rotational motion 2 ;

$$
V_{\text {int }}^{\mathrm{eff}}(t)=-\frac{1}{4} \sum_{\rho \rho^{\prime}} \alpha_{\rho \rho^{\prime}}^{\mathrm{LF}}(\theta, \varphi, \chi) \varepsilon_{\rho}(t) \varepsilon_{\rho^{\prime}}^{*}(t) .
$$

The angular dependence of the interaction potential is contained in the anisotropy of the molecular polarizability tensor $\hat{\alpha}^{\mathrm{LF}}(\theta, \varphi, \chi)$ that could be expressed in terms of the molecular-fixed frame polarizability tensor $\hat{\alpha}^{\mathrm{MF}}$ as

$$
\alpha_{\rho \rho^{\prime}}^{\mathrm{LF}}(\theta, \varphi, \chi)=\sum_{k k^{\prime}}\langle\rho \mid k\rangle \alpha_{k k^{\prime}}^{\mathrm{MF}}\left\langle k^{\prime} \mid \rho^{\prime}\right\rangle .
$$

Here, indexes $k$ and $k^{\prime}$ go over the molecular-fixed coordinates $X, Y, Z$, and $\langle\rho \mid k\rangle$ are the directional cosines between the laboratory-fixed and molecularfixed frames unit vectors, which contain all the angular dependence of the interaction potential. The final expression for the effective potential is then:

$$
V_{\text {int }}^{\mathrm{eff}}(t)=-\frac{1}{4} \sum_{\rho \rho^{\prime} k k^{\prime}}\langle\rho \mid k\rangle \alpha_{k k^{\prime}}^{\mathrm{MF}}\left\langle k^{\prime} \mid \rho^{\prime}\right\rangle \varepsilon_{\rho}(t) \varepsilon_{\rho^{\prime}}^{*}(t) .
$$

An illustrative example of potential $(1.10)$ is the case of a field, linearly polarized along $z$. The unit polarization vector $\hat{\varepsilon}$ is equal to $\hat{z}$ in this case, and if we choose the molecular-fixed unit vectors $\hat{X}, \hat{Y}, \hat{Z}$ to be the principal axes of the polarizability tensor $\hat{\alpha}^{\mathrm{MF}}$, with $\hat{Z}$ chosen along the direction of maximal polarizability, the interaction potential becomes 1$]$ :

$$
V_{\text {int }}^{\mathrm{eff}}(t)=-\frac{1}{4} \varepsilon^{2}(t)\left(\Delta \alpha_{Z X} \cos ^{2} \theta+\Delta \alpha_{Y X} \sin ^{2} \theta \sin ^{2} \chi\right)
$$

where $\Delta \alpha_{k k^{\prime}}=\alpha_{k k}^{\mathrm{MF}}-\alpha_{k^{\prime} k^{\prime}}^{\mathrm{MF}}$. This potential has a global minimum at $\theta=0$, independent of $\phi$ and $\chi$, which means that it is energetically preferable for a molecule to align its axis of maximal polarizability along the polarization direction.

Most of the methods that use the interaction potential (1.10) to control the molecular rotational state could be categorized into two large classes, namely adiabatic and non-adiabatic methods, depending on the shape of the envelope $\varepsilon(t)[36,119]$. The two approaches are illustrated in Fig. 1.1.

\footnotetext{
${ }^{2}$ Up until now we assumed that the molecular permanent dipole moment $\boldsymbol{\mu}_{\text {perm }}$ is zero. For a non-zero $\boldsymbol{\mu}_{\text {perm }}$ an additional term $-\boldsymbol{\mu}_{\text {perm }} \cdot \mathbf{E}(t)$ would need to be included in the interaction potential. This term, however, would disappear when averaged over the field oscillations.
} 
(a)

Field-free J

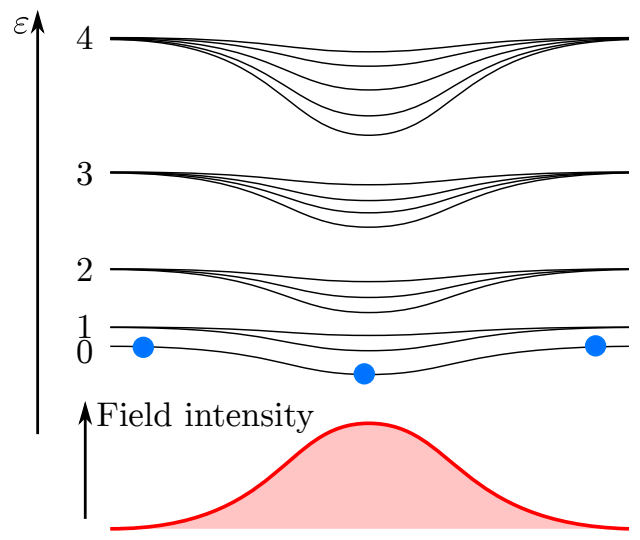

(b)

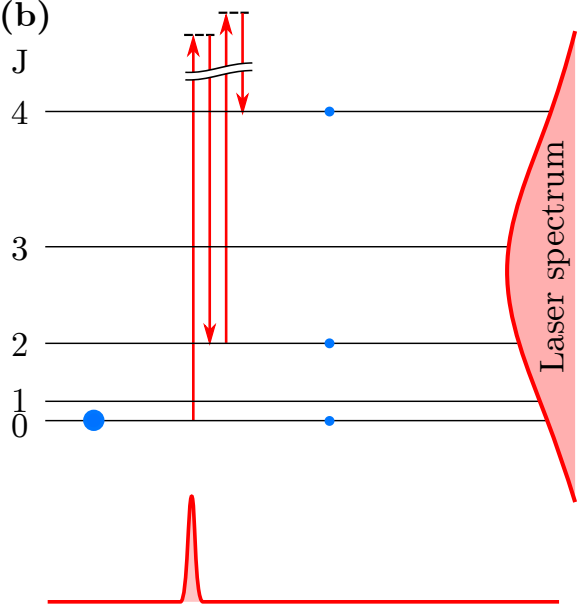

Time

Figure 1.1: Illustration of (a) adiabatic vs. (b) non-adiabatic rotational excitation.

Adiabatic methods, usually used to achieve alignment[74, 113, 119, employ $\varepsilon(t)$ that changes slowly on the time scale of the rotational period, adiabatically modifying the rotational states (Fig. 1.1 (a)). If we take a molecule in a rotational ground state and slowly turn a linearly polarized laser field on, the molecule will end up near the bottom of the potential well (1.11), having its axis of maximal polarizability aligned along the field direction. More complicated schemes involve elliptically polarized light for aligning all three axes of a molecule[77]. The adiabatic methods are suffering from two limitations. First, the control is executed only during the laser pulse, with the molecules adiabatically returning to their initial rotational state when the laser field is gone. The presence of a high intensity field is undesirable in many experimental scenarios. Second, the adiabatic methods can only be used to achieve alignment or orientation, while other kinds of rotational control remain inaccessible to them.

A different, non-adiabatic approach employs fields, whose envelops $\varepsilon(t)$ change rapidly on the scale of molecular rotational periods. A famous example, the non-adiabatic "delta-kick" excitation, is when the molecules are irradiated by a very short intense linearly polarized pulse, usually on a femtosecond scale. Unlike the adiabatic case, the molecules are essentially frozen during the pulse, but experience a "kick" towards the direction of the polar- 
ization vector. Quantum mechanically this corresponds to many rotational states being populated via multiple Raman transitions, with both pump and Stokes photons coming from the same laser field (Fig. 1.1(b)). Conventional single-pulse excitation schemes lack selectivity with respect to the final speed of molecular rotation and produce broad rotational distributions [112]. Although sequences of pulses have been successfully used for selective[17, 28, 103, 143] and directional [29, 66, 142] rotational excitation, the range of accessible rotational states has been limited to relatively low quantum numbers (of order 10 above the initial state) due to the molecular breakdown in intense laser fields.

\subsection{Optical centrifuge}

Another approach to the rotational control of linear molecules, combining the advantages of both adiabatic and non-adiabatic techniques, was proposed in [57]. A linearly polarized intense laser field slowly turns on, aligning the molecular axes of maximal polarizablity (typically, the internuclear axis of a linear molecule) along its direction. The polarization plane then undergoes an accelerated rotation illustrated in Fig. 1.2 (a), adiabatically spinning the molecular axes along and eventually releasing them in high rotational states.

Consider two laser fields, circularly polarized in the opposite directions, of the same amplitude $E_{0}$ and slightly different optical frequencies $\omega_{-}$and $\omega_{+}, \omega_{+}>\omega_{-}$. Provided both fields are propagating along the $z$ direction, the electric fields that each of them produces in the $x y$ plane, can be written as

$$
\varepsilon_{-}=\varepsilon_{0}\left(\begin{array}{c}
\sin \omega_{-} t \\
\cos \omega_{-} t
\end{array}\right), \varepsilon_{+}=\varepsilon_{0}\left(\begin{array}{c}
\sin \omega_{+} t \\
-\cos \omega_{+} t
\end{array}\right) .
$$

The total electric field is then:

$$
\varepsilon=\varepsilon_{-}+\varepsilon_{-}=\varepsilon_{0}\left(\begin{array}{c}
\sin \omega_{-} t+\sin \omega_{+} t \\
\cos \omega_{-} t-\cos \omega_{+} t
\end{array}\right)=2 \varepsilon_{0} \sin \omega_{0} t\left(\begin{array}{c}
\cos \Omega t \\
\sin \Omega t
\end{array}\right),
$$

with $\omega_{0}=\frac{\omega_{+}+\omega_{-}}{2}$ and $\Omega=\frac{\omega_{+}-\omega_{-}}{2}$. Provided the fields have close optical frequencies, $\Omega \ll \omega_{0}$, this expression describes a linearly polarized field of frequency $\omega_{0}$ with a plane of polarization precessing around the propagation direction with an angular frequency $\Omega$.

To achieve the field configuration of Fig. 1.2 (a), it is therefore enough to add together two circularly polarized laser pulses, whose optical frequencies change linearly in time, starting at the same value $\omega_{0}, \omega_{ \pm}(t)=\omega_{0} \pm \beta t$ 

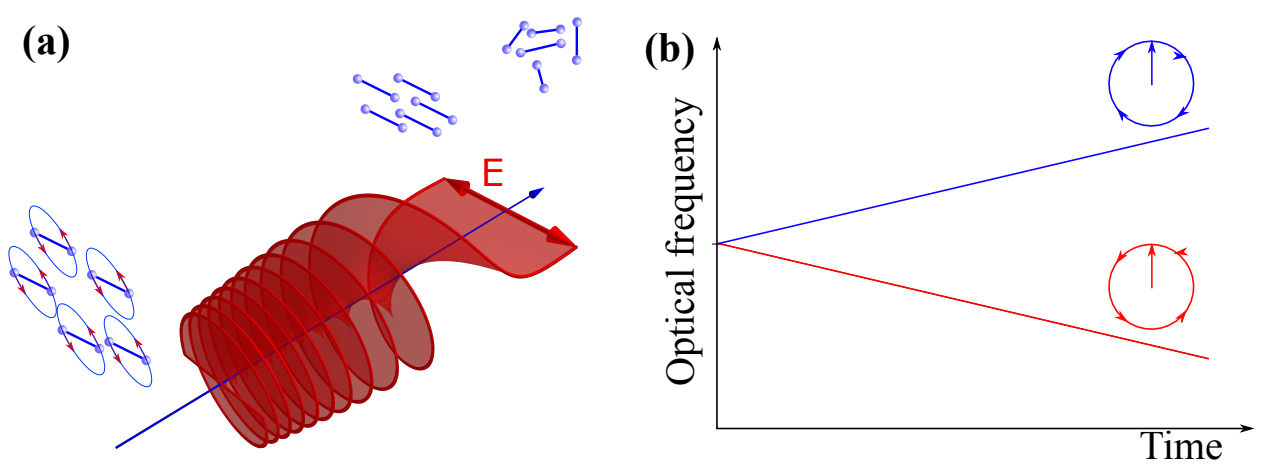

Figure 1.2: (a) Principle of an optical centrifuge excitation. A linearly polarized intense non-resonant laser field aligns the molecular axes along its polarization direction. This direction then undergoes accelerated rotation, sweeping the depicted red "corkscrew" shape and dragging the molecular axes along, eventually releasing them to freely rotate with a high angular frequency. (b) To produce the centrifuge field, one can combine two circularly polarized pulses of the opposite chirp and polarization handedness, shown in the figure.

(Fig. 1.2 (b)). Such frequency chirped pulses can be easily produced by the femtosecond pulse shaping techniques, as described in subsection 2.1.2. The highest angular frequency $\Omega_{\max }$, that the molecules acquire during the centrifugal excitation, corresponds to half the laser bandwidth and reach $6 \times 10^{13} \mathrm{rad} / \mathrm{s}$ for currently available broadband laser systems. In diatomic oxygen, this frequency corresponds to the angular momentum of $\sim 120 \hbar$, in contrast to $10-20 \hbar$ typically achievable with non-adiabatic methods.

Quantum mechanical description of the optical centrifuge was given by Vitanov and Girard[131]. Let us consider a diatomic molecule initially in the ground rotational state $J=0, M_{J}=0$, placed in a field of $n_{-}$and $n_{+}$photons of the positively and negatively chirped centrifuge components, respectively. The molecule can absorb a photon from the lower frequency pulse field, and emit it to the higher frequency one. The system is now described by a "dressed state" with an angular momentum of 2 , and the photon fields population of $\left(n_{-}+1\right)$ and $\left(n_{+}-1\right)$. Since each of the photons bears an angular momentum of $\hbar$, the magnetic quantum number would also increase by $2, M_{J}=2$. The process could then be repeated, leading to the excitation of $J=4$ state, then $J=6$ state and so on. The centrifuge field therefore couples the states $\left|J, M_{J}=J, n_{-}+J / 2, n_{+}-J / 2\right\rangle$. 


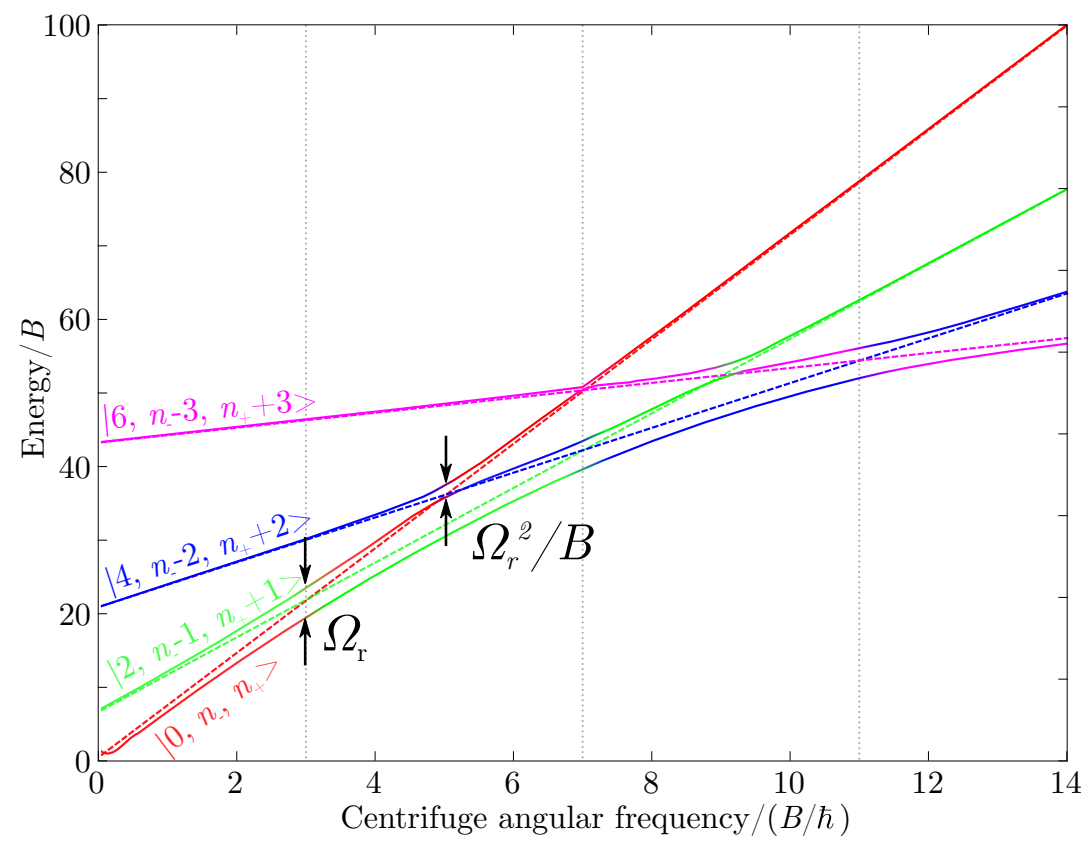

Figure 1.3: Illustration of an adiabatic population transfer in the optical centrifuge.

The energies of these states in the absence of the interaction between the molecule and the field are given by a simple sum of the energy of the field and the rotational energy of the molecule:

$$
\begin{array}{r}
\epsilon\left(J, n_{-}+J / 2, n_{+}-J / 2\right)=B J(J+1)+\hbar\left(n_{-}+\frac{J}{2}\right) \omega_{-}+\hbar\left(n_{+}-\frac{J}{2}\right) \omega_{+} \\
=B J(J+1)-\hbar \Omega J+\text { const }
\end{array}
$$

where const is a constant independent of the rotational state. These energies are plotted as a function of the centrifuge rotational frequency $\Omega$ in Fig. 1.3 with dashed lines.

If we turn on the interaction between the field and the molecular rotational motion, described by the Raman Rabi frequency $\Omega_{\mathrm{r}}=\frac{\varepsilon^{2} \Delta \alpha}{8}[112$, the neighboring terms with $\Delta J=2$ would repel (solid lines in Fig. 1.3), forming a network of avoided crossings at $\Omega=\Omega_{n} \equiv(4 n-1) B / \hbar, n=1,2, \ldots$, 
emphasized by the grey dashed lines in Fig. 1.3. The level separation at the crossings is $\Delta \epsilon=\Omega_{\mathrm{r}}$. If $\Omega$ grows slowly in time, fulfilling the adiabaticity condition $\dot{\Omega} / \Omega \ll \Omega_{\mathrm{r}}$, the molecule, initially in the ground rotational state, would follow the lowest solid line in Fig. 1.3 , consecutively passing the rotational states $|J=0\rangle \rightarrow|J=2\rangle \rightarrow \ldots$.

One can notice from Fig. 1.3 , that starting from $|J=2\rangle$, the molecule can also adiabatically climb the rotational states ladder, this time via $|J=2\rangle \rightarrow$ $|J=0\rangle \rightarrow|J=4\rangle \rightarrow|J=2\rangle \rightarrow|J=6\rangle \rightarrow \ldots$ The "forbidden" $\Delta J=4$ Raman transitions are allowed because the dressed states are superpositions of several field-free $J$-states. The strength of such transition is much lower, corresponding to a level splitting of $\Delta \epsilon=\Omega_{\mathrm{r}}^{2} / B$. Similar splittings with even smaller values of $\Delta \epsilon$ occur for higher $J$-states. The adiabaticity condition is harder to satisfy in this case, which means that if the field intensity is not high enough, or the rotational frequency ramps too quickly, part of the initially rotationally excited population can hop over the avoided crossing, escaping the process of further acceleration.

In the classical picture, when the field is turned on, the field free rotational states are adiabatically transfered to the eigenstates of the angular potential well (1.11), the so-called pendular states[36]. In such states, molecular axes are aligned with the field polarization. Different initial rotational states would adiabatically correspond to different pendular states, with the pendular energy in general increasing with field free $J$. The effect of a nonadiabatic escape of the molecules from the accelerating centrifuge in this language is attributed to their "spilling" from the levels that lie too close to the angular potential top, if this potential accelerates too quickly or is not deep enough.

\subsection{Goals of this work}

Since the original theoretical proposal, the technique of an optical centrifuge was experimentally realized by two groups. Villeneuve et al.[130. observed the dissociation of the centrifuged chlorine molecules, which was attributed to bond breaking due to the centrifugal forces. Yuan et al. 138 observed substantial heating of the centrifuged molecular gases as the extremely high rotational energy was distributed to other degrees of freedom. In both cases, indirect secondary processes were used to detect rotational excitation. To study the properties of the centrifuge excitation and to explore the possibility of controlled molecular spinning, one needs to develop direct methods of detecting extreme rotational states. Two different approaches of character- 
izing molecular rotation are available. The first one is based on either time or frequency resolved spectroscopy, whereas the second one involves direct imaging of the molecular angular distribution.

There are many spectroscopic techniques suitable for studying the rotational structure. Although purely rotational absorption spectroscopy is possible, for typical molecules it would require microwave or terahertz sources, tunable in wide frequency ranges specific to each molecule of interest. Using non-resonant Raman instead of a resonant absorption process lifts the requirement for a molecule-specific source. Time-resolved coherent Raman scattering also provides information about the relative phase of the rotational states. As a third-order nonlinear optical process, however, it requires high molecular densities.

An alternative to a purely rotational spectroscopy is vibrational or electronic spectroscopy with rotational resolution. Yuan and coworkers [138] used infra-red (IR) spectroscopy to detect super rotors, but their experimental system only allowed to see relatively low energy levels. Resonanceenhanced multiphoton ionization (REMPI) spectroscopy [81], on the other hand, proved to be a very sensitive tool and is routinely used to measure rotational state distribution in molecules [122]. Its applicability to super rotors was unclear, as the excited electronic states, participating in the ionization process, is often poorly known at high degree of rotational excitation.

The above mentioned spectroscopic methods provide limited information about the relative phases of the rotational states and the distribution among degenerate $M_{J}$ levels. More complete information could be acquired from the direct measurement of molecular angular distributions. Such methods, often used for detecting molecular alignment [27], could be applied to the study of molecular super rotors.

A method for controlling the magnitude of angular momentum, acquired by the molecules in the optical centrifuge, was proposed in the first experimental work on the subject[130]. The authors demonstrated that truncating the centrifuge spectrum resulted in the decrease of the centrifuge-induced angular momentum, reflected by the disappearance of the spinning-induced dissociation below some threshold value of the laser bandwidth. The employed detection method, however, did not allow them to study the limits and properties of the centrifuge-based rotational control, which remained an open area for further investigation.

In addition to the magnitude, the methods of controlling the direction of molecular rotation are equally desired. Simple reorientation of the $J$ vector is possible by changing the laser propagation direction in a broad range of angles, which may be technically challenging in many experimental scenar- 
ios. Yun and coworkers [140] theoretically proposed a method of controlling the direction of the angular momentum of non-magnetic molecules through the interaction of magnetic field with the rotationally induced magnetization. The applicability of such magnetic control to both non-magnetic and paramagnetic super rotors required further experimental studies.

In general, full rotational control implies redistributing the molecular population over the magnetic quantum numbers $M_{J}$, rather than a simple increasing of $\mathrm{J}$. As discussed above, $M_{J}=J$ is dictated by the selection rules $\Delta M_{J}=2$ for the Raman process with two circularly polarized photons at each step of the rotational ladder climbing. This suggests, that modifying the polarization of the centrifuge could provide yet another means for controlling molecular rotation.

The importance of the adiabaticity of the centrifuge excitation was realized in the original paper of Karczmarek and coworkers [57]. Further theoretical studies of Vitanov and Girard [131] suggested that the deviation from the complete adiabaticity would result in a broadened coherent rotational state distribution, leading to the wavepacket time evolution. The dynamics of the wavepackets consisting of rotational states with $M_{J}=J$ was theoretically studied in the context of so-called "cogwheel" states [76] - the states that propagate without dispersion along classical trajectories. Cryan and coworkers 18 proposed to use such states as a "stopwatch" to synchronize ultrafast laser pulses, with the classical rotation of molecular axes playing the role of a clock's hand. One could, however, expect that the uncertainty principle puts a limit on the classical molecular rotation. Applicability of the centrifuge to producing dispersionless states and the dynamical properties of the created wavepackets were yet to be studied experimentally.

Recently, much effort and resources has been invested in controlling the degree of molecular alignment[119]. As discussed above, the two main approaches are based on adiabatic and non-adiabatic excitation schemes. Adiabatic approach was shown very effective, producing high-degree alignment in various molecular species $[63,64]$. Its main drawback is the presence of the intense external field during the alignment window, which is intolerable in many cases. Adiabaticity is even more desirable for the alignment of asymmetric top molecules[74]. The three different moments of inertia lead to an irregular rotational spectrum. The wide rotational distributions produced by non-adiabatic methods therefore result in complex aperiodic dynamics, which severely complicates the routes to rotational control. Combining adiabaticity with the field-free excitation, the methods based on the optical centrifuge could open new possibilities for reaching strong and permanent alignment of asymmetric molecules. 
Collisional relaxation of super rotors was studied both theoretically $[33$, 42 and experimentally [138. Three stages of such relaxation - decoherence, reorientation and thermalization, were identified. However, the experimental methods based on the rotational excitation with a short femtosecond pulse or indirect rotational detection, provided limited information about the decay dynamics and were not capable of characterizing these decay stages separately. Different detection techniques were also required to address other theoretical works which suggested a number of exotic effects in a gas of molecular super rotors. These effects include vortices formation [120], explosive thermalization and anisotropic diffusion [62].

The main goal of this work was to build an optical centrifuge for producing molecular super rotors and to experimentally address all the open questions listed above. 


\section{Chapter 2}

\section{Experimental methods}

To experimentally investigate molecular super rotors, two ingredients were required. First, we needed a laser and an optical system to produce the centrifuge field capable of exciting the molecular ensemble to high rotational states. Second, a detection setup was required to detect and analyze the behavior of molecules in those states.

An optical centrifuge setup was the same for all of our experiments and is described in section 2.1. The detection methods we used can be divided into two classes, applied to gases under ambient conditions (at room temperature, and pressures of order of 1 bar, Raman setup) and to dilute cold gases (UHV setup).

Rotational relaxation of molecular super rotors in dense room-temperature gases is of great interest in the context of molecular kinetics. To investigate the effects of super rotation on various relaxation processes, a hermetic chamber, filled with a few hundred Torr of gas under study, was used. The main technique utilized in this setup for the rotation detection was coherent Raman spectroscopy. Being a spectroscopic method, it allowed a sensitive measurement of the rotational state distributions in a single laser shot. With the use of circularly polarized probe beam, it provided the information on the directionality of molecular rotation. Finally, since Raman scattering does not directly rely on electronic resonances, it could be applied to almost any molecular system without any modifications. This method is described in details in section 2.2.

In many cases, however, initially cold gas ensembles were desired. Studies of the processes taking place on long time scales required the absence of collisions. In the case of asymmetric top molecules, where at room temperature the initial thermal excitation of a broad rotational wave packet led to overcomplicated dynamics, low temperature was essential. Finally, ion detectors, utilized for imaging the molecular angular distributions, require high-vacuum conditions to operate. For these experiments an ultra-high vacuum (UHV) chamber was built. A jet of gas, expanded through a narrow nozzle from high pressure to vacuum, served as a molecular source. During this expansion, both the translational and the internal temperature of gas 
decreased dramatically, typically below $10 \mathrm{~K}$, resulting in the collisionless dynamics and a very narrow rotational distribution. This was accompanied by a dramatic (roughly estimated at around 8 orders of magnitude) drop in the molecular number density. Since the coherent Raman scattering signal depends quadratically on the molecular density, it becomes inapplicable in the UHV setup, and other detection techniques were required.

One such technique, Resonance Enhanced Multiphoton Ionization (REMPI) spectroscopy (section 2.3), uses a tunable ultraviolet laser to ionize the molecular sample while the produced ion current is measured as a function of the UV wavelength. Positions and intensities of the resonances allow to determine the molecular rotational population prior to ionization. Unlike Raman spectroscopy, it is molecule specific and requires the precise knowledge of the molecular energy spectrum. Moreover, it requires long scans of the laser wavelength to determine the population. The main advantage of this method is its high sensitivity, down to a few ions with a modern ion detectors, such as micro-channel plates (MCP), which makes it suitable for the spectroscopy of dilute gas samples in the UHV setup.

Both methods described above enabled us to measure the rotational level population. The phase information was completely lost in the case of REMPI, and limited in the case of Raman spectroscopy. Moreover, the distribution among degenerate states, such as the states with different projections of the molecular angular momentum, cannot be fully determined by these methods. An alternative approach, allowing for a complete reconstruction of the rotational wave function, is based on detecting the timedependent instantaneous angular distribution of molecules. In practice, this is typically achieved by breaking the molecules apart with an ultrashort laser pulse, and measuring the recoil direction of the fragment ions using the so-called Velocity-Map Imaging (VMI) technique, described in details in section 2.4.

\subsection{Optical centrifuge}

\subsubsection{Laser system}

The main laser system, used in our experiments to produce the field of an optical centrifuge, was a commercially available femtosecond source, shown in Fig. 2.1. Titanium-Sapphire based oscillator (Coherent Micra) generated spectrally broad, $90 \mathrm{~nm}$ full-width at half maximum (FWHM) laser pulses, centered at $800 \mathrm{~nm}$ at $80 \mathrm{MHz}$ repetition rate (Fig. 2.1 (b), red line). As the energy of each pulse was a mere $5 \mathrm{~nJ}$, and because the centrifuge re- 


\subsection{Optical centrifuge}

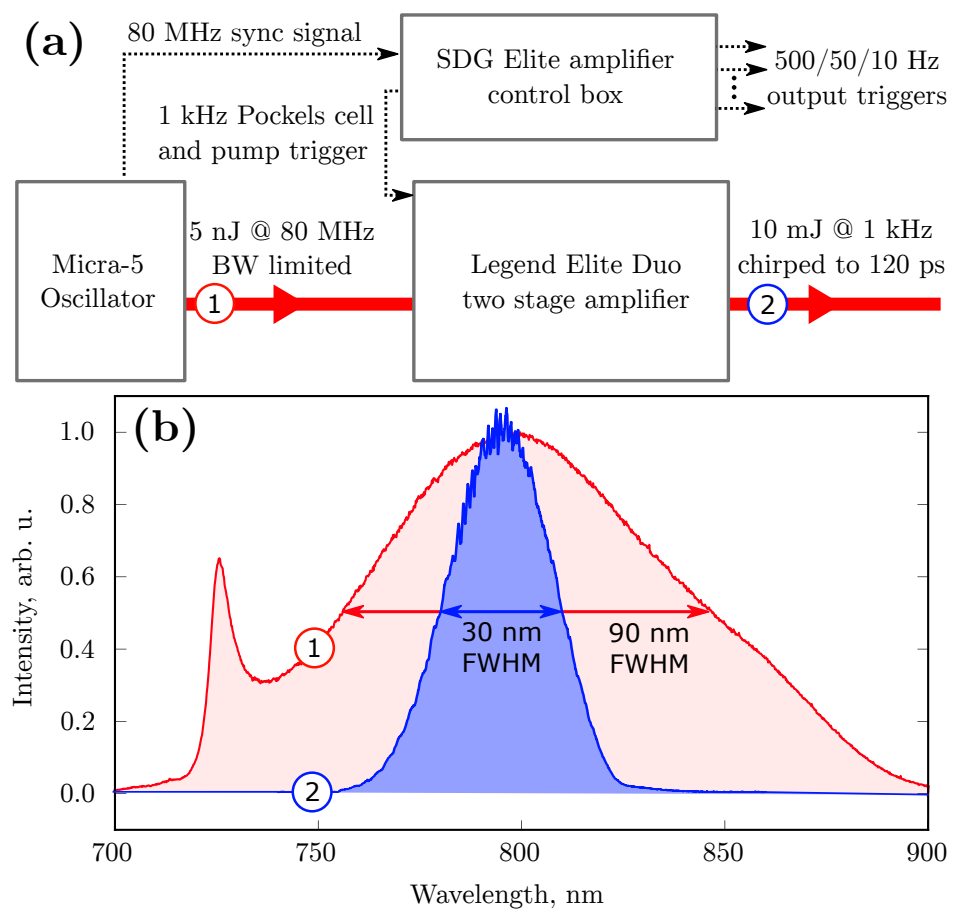

Figure 2.1: (a) Diagram of our femtosecond laser system. (b) Spectra of laser pulses before (red line) and after (blue line) amplification.

quired intense laser fields, it was amplified in a Titanium-Sapphire two stage chirped-pulse amplifier (Coherent Legend Elite Duo), operating at $1 \mathrm{kHz}$ repetition rate. The amplified bandwidth decreased to $30 \mathrm{~nm}$ (Fig. 2.1 (b), blue line), which corresponds to a 35 fs long Fourier transform-limited pulse. The energy and duration of each pulse was $10 \mathrm{~mJ}$ and $120 \mathrm{ps}$, respectively.

\subsubsection{Centrifuge shaper}

In the heart of our experimental setup was an optical centrifuge shaper, which was built according to the original proposal of [130] to create an optical field configuration of Fig. 1.2 (b). The shaper diagram is shown in Fig. 2.2 (a). The concept is based on a common 4 -f optical shaper setup, where the input diffraction grating G0 is followed by a pair of identical positive lenses L0 and L1 and a grating G1. The beam passed through the shaper in one direction, and was turned back by a retroreflector RR1, missing the input mirror IM on its way out. The effect of such shaping was 

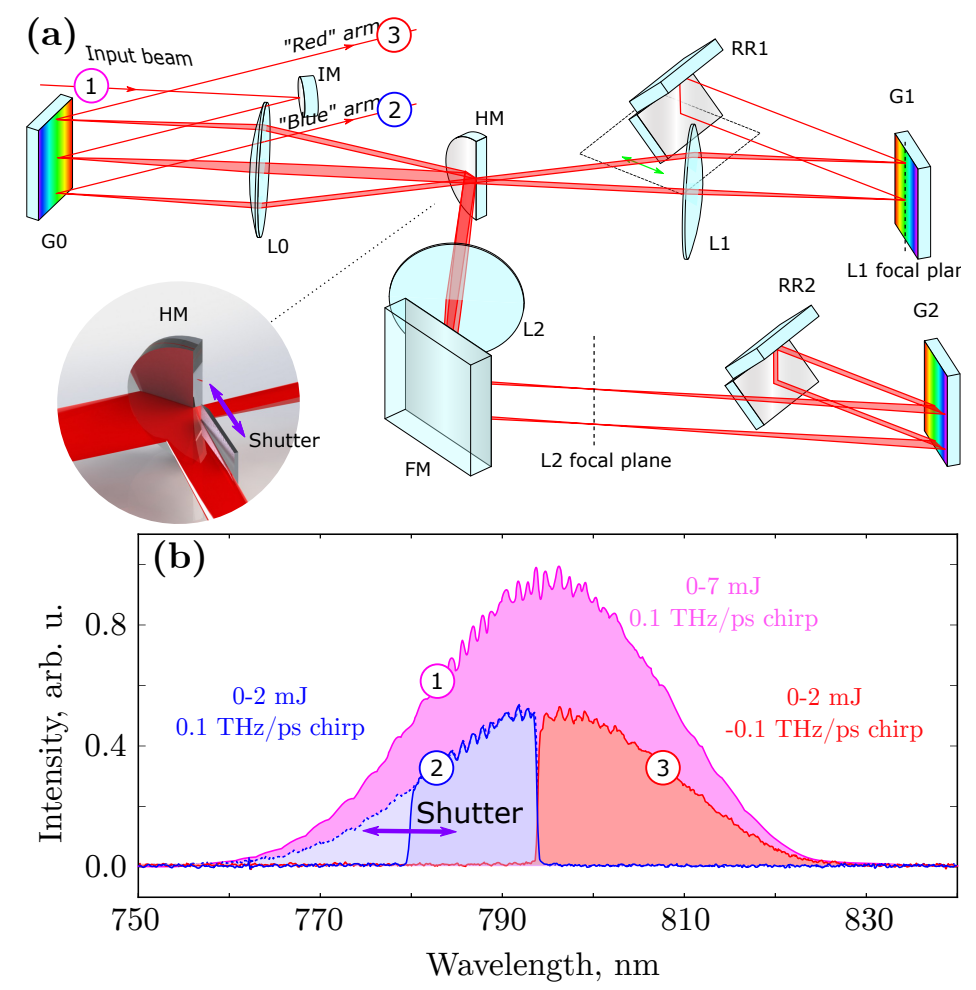

Figure 2.2: (a) Optical centrifuge shaper. IM: input mirror, G0, G1, G2: diffraction gratings, L1, L2, L3: $f=250 \mathrm{~mm}$ achromatic lenses, HM: halfcut mirror, FM: folding mirror, RR1, RR2: retroreflectors. Inset: movable shutter. (b) Laser spectra before (magenta), and after the shaping for the "blue" (blue) and "red" (red) arms. The "blue" arm spectrum is shown with the shutter completely out of (dashed), and half way in the beam (solid)

an addition of a linear chirp, proportional to the distance from the output grating to the focal plane of the second lens (L1). Putting a half mirror HM in a common focal plane of the two lenses on the optical axis, where the wavelength was directly mapped to a horizontal position, we reflected the long-wave half of the initial beam towards its own combination of lens L2, grating G2 and retroreflector RR2. This configuration allowed us to control the chirps of the two spectral halves individually: while G1 was placed at L1's focal plane, keeping the chirp of the "blue" part of the spectrum unchanged at $0.1 \mathrm{THz} / \mathrm{ps}, \mathrm{G} 2$ was shifted so as to invert the chirp of the "red" part. 
Prior to shaping, the laser beam width was decreased by a factor of 2 from $12 \mathrm{~mm}$ to $6 \mathrm{~mm}$ with a telescope, which was necessary for fitting the beams through the shaper optics.

The output of the centrifuge shaper consisted of two oppositely chirped beams, "red" and "blue" arms, whose spectra are shown in Fig. 2.2 (b) together with the input spectrum (purple). By putting a partial beam shutter in the Fourier plane of the shaper and moving it in and out of the beam path, as shown in the inset of Fig. 2.2 (a), we were able to control the bandwidth of the "blue" arm, controlling the centrifuge terminal angular frequency. The shutter was mounted on a $25-\mathrm{mm}$ travel motorized translation stage (Newport MFA-CC), computer controlled for precise positioning.

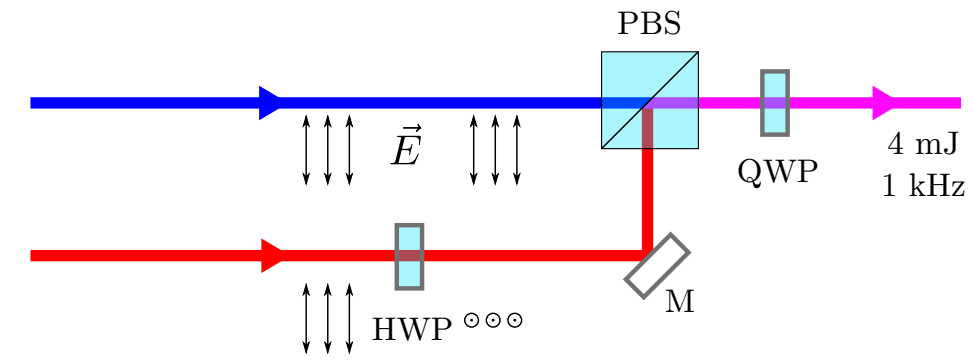

Figure 2.3: Combining the centrifuge arms. HWP: half-wave plate, M: mirror, PBS: polarizing beam splitter cube, QWP: quarter-wave plate.

To create a rotating field of an optical centrifuge, the two arms had to be circularly polarized and combined together. This was executed with a polarizing beam splitter cube and a quarter-wave plate, as shown in Fig. 2.3, producing an optical centrifuge beam of $4 \mathrm{~mJ}$ per pulse at $1 \mathrm{kHz}$ repetition rate. For some experiments, however, higher pulse energies were required. In this case an additional custom-built multi-pass amplifier (MPA, see subsection 2.2.2) was used to amplify the two centrifuge arms. The amplification had to be done individually prior to joining them up, due to the polarization sensitivity of the amplification process.

\section{Shaper adjustment}

The centrifuge beam was carefully characterized and adjusted in the spatial, spectral and time domains.

To adjust the spatial characteristics of the beams, their profiles were observed both in the near field, right at the output of the shaper, as well 

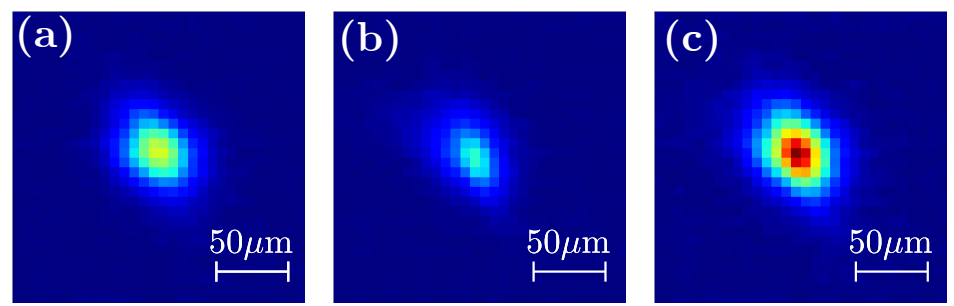

Figure 2.4: Far field profiles of the (a) "blue" arm, (b) "red" arm, (c)both beams combined.

as in the far field. In the near field the beams were observed on a piece of paper, and the inspection usually boiled down to verifying that they were not clipped in the shaper. Adjustment in the far field required much finer control, as the beams profiles there were more sensitive to the spherical aberrations in and misalignment of the shaper lenses, and to the spatial chirps introduced by the gratings. To do that, the beams were focussed with a $\mathrm{f}=250 \mathrm{~mm}$ lens and inspected with a beam profiler (Coherent LaserCam$\mathrm{HR}$ ). Lenses L1 and L2 were then positioned so as to achieve the best circularity of the beam profiles and simultaneous focussing of the two arms. The examples of the optimal beam profiles are shown in Fig. 2.4.

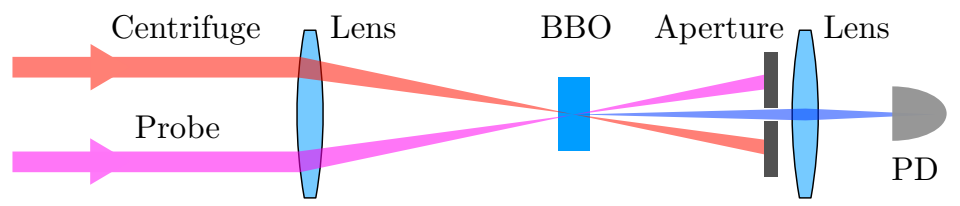

Figure 2.5: Illustration of the standard cross-correlation setup.

A quicker way of overlapping the two centrifuge arms, used routinely on a day-to-day basis, was implemented in a UHV setup. The centrifuge pulse energy was high enough to cause a detectable multi-photon ionization from the molecules in the chamber (see section 2.2). The corresponding ion current was measured as the centrifuge output mirrors were adjusted to maximize it. Owing to the high nonlinearity of the ionization process, the highest current was observed at the best overlap of the two beams.

To characterize the temporal profile of the centrifuge pulses, cross-correlation measurements were carried out (Fig. 2.5). The centrifuge and probe beams were aligned collinearly and focussed together on a $\mathrm{BaB}_{2} \mathrm{O}_{4}(\mathrm{BBO})$ non- 
linear crystal. The sum frequency signal, generated through the nonlinear mixing of the two fields, was recorded as a function of the probe delay.

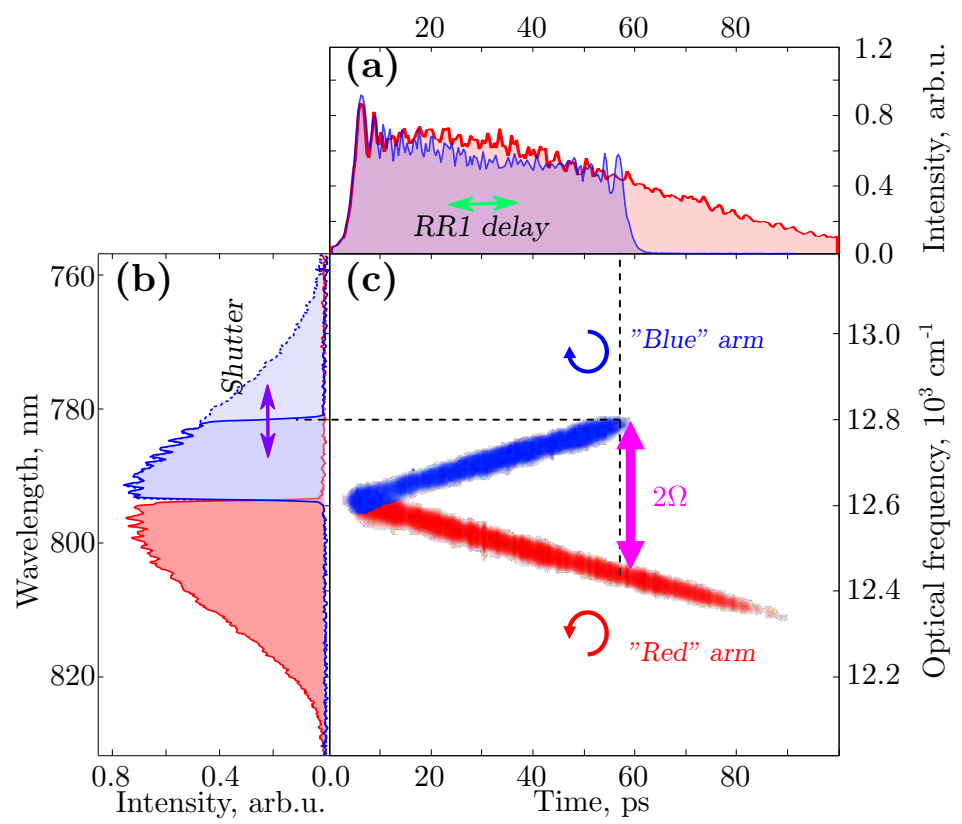

Figure 2.6: Time- and frequency-resolved characterization of the centrifuge beams. In each panel, the measurements related to the "blue" ("red") arm are colored blue (red). (a) Time-resolved cross correlation measurements. (b) Spectra of the beams. (c) Time-resolved spectrogram of each centrifuge arm. Tilted lines correspond to a frequency chirp.

Two kinds of measurements were carried out using this setup. The first one was executed with a short 40 fs probe, and the sum frequency was detected with a photodiode (Thorlabs). The electrical signal from the photodiode was integrated on a gated integrator (SRS SR250) and recorded with a data acquisition board (National Instruments 6024E). The resulting signal (Fig. 2.6 (a)) represented the time dependence of the centrifuge arms taken with a high temporal resolution. Having taken the signal from the blue and the red arms separately, we were able to adjust the blue arm delay (RR1 position, see Fig. 2.2 (a)), synchronizing it with the red arm.

To characterize the centrifuge in the frequency domain, the probe bandwidth was narrowed and the sum frequency signal was measured with a spectrometer (Photon Control). The resulting spectrogram, shown in Fig. 2.6 (c) 
after the probe photon energy subtraction, provided a way of measuring the chirps of the two arms.

\section{$2.2 \quad$ Raman setup}

Time-resolved coherent Raman scattering is a common tool of choice for analyzing the dynamics of molecular rotation. It has been successfully used for the precision thermometry of flames[109] and the studies of collisional decoherence in dense gas media 60,90 . We employed this technique to detect and study centrifuged molecules in dense gas samples.

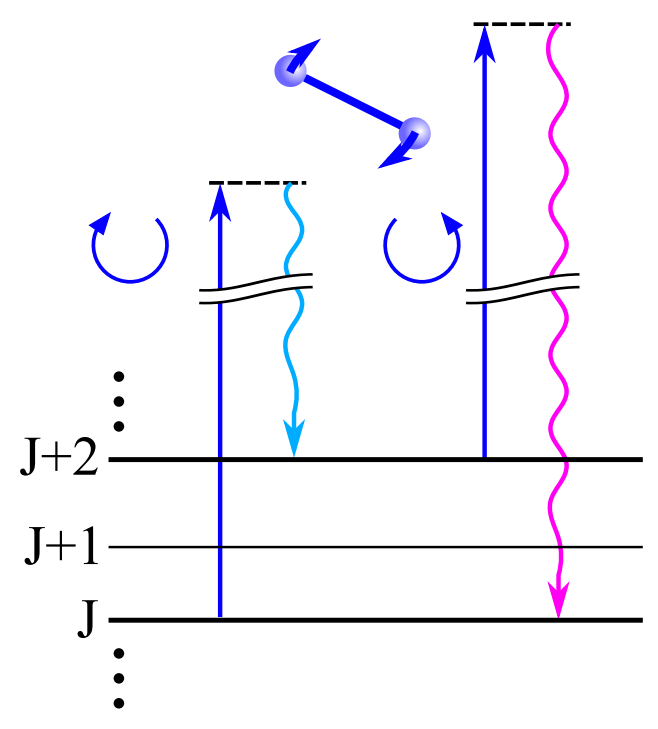

Figure 2.7: An illustration of Stokes (left) and anti-Stokes (right) rotational Raman scattering.

Quantum mechanically, synchronous molecular rotation corresponds to a superposition of a few rotational quantum states - a "rotational wave packet", with an average frequency separation matching the frequency of the classical rotation. Consider two coherently populated rotational states $\left|J, M_{J}=J\right\rangle$ and $\left|J+2, M_{J}=J+2\right\rangle$ (Fig. 2.7). Laser light can coherently scatter off of it, producing either Stokes (left diagram) or anti-Stokes (right diagram) radiation, detuned from the initial frequency by the energy separation of the relevant rotational levels. Owing to the well-defined angular momentum projection difference $\left(\Delta M_{J}=2\right)$ between the two states, a circu- 
larly polarized light will acquire Stokes or anti-Stokes frequency sidebands, depending on whether the molecules rotate in the same or opposite direction with respect to the probe polarization. In both cases, illustrated in Fig. 2.7, the magnitude of the Raman shift equals twice the rotation frequency, while its sign reflects the direction of molecular rotation.

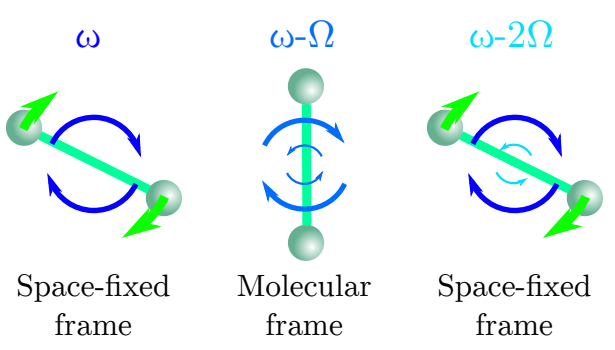

Figure 2.8: An illustration of the rotational Doppler effect. Thick (thin) blue arrows show the circularly polarized probe field of the same (opposite) direction with respect to the molecular rotation (green arrows).

In classical terms, the frequency shift can also be viewed as a result of the rotational Doppler effect 68] (Fig. 2.8). Indeed, consider a classical molecule, co-rotating with the probe field of frequency $\omega$. In the frame of reference connected to the molecule, the observed light frequency will be lower by the angular frequency of rotation, $\Omega$, due to the Doppler effect. Anisotropic polarizability of the molecule will introduce birefringence, adding ellipticity to the circularly polarized probe. This ellipticity is equivalent to the appearance of a weak field of the same frequency $\omega-\Omega$, circularly polarized in the opposite direction. Transforming this field back to the laboratory frame will further decrease its frequency by $\Omega$ (since now it is counter-rotating with the molecule) to $\omega-2 \Omega$, in good agreement with the quantum picture of coherent Raman scattering, described above. Similar arguments justify the appearance of a $\omega+2 \Omega$ sideband in the case, when the rotation direction of the molecule coincides with that of the probe field.

\subsubsection{Raman chamber}

To carry out a number of experiments on molecular super rotors under ambient conditions, we built a simple gas chamber shown in Fig. 2.9. The chamber had a barrel shape, with a large $12^{\prime \prime}$ opening on top, used to mount a wide acrylic viewport, and four $2.75^{\prime \prime}$ conflat side flanges. 


\subsection{Raman setup}

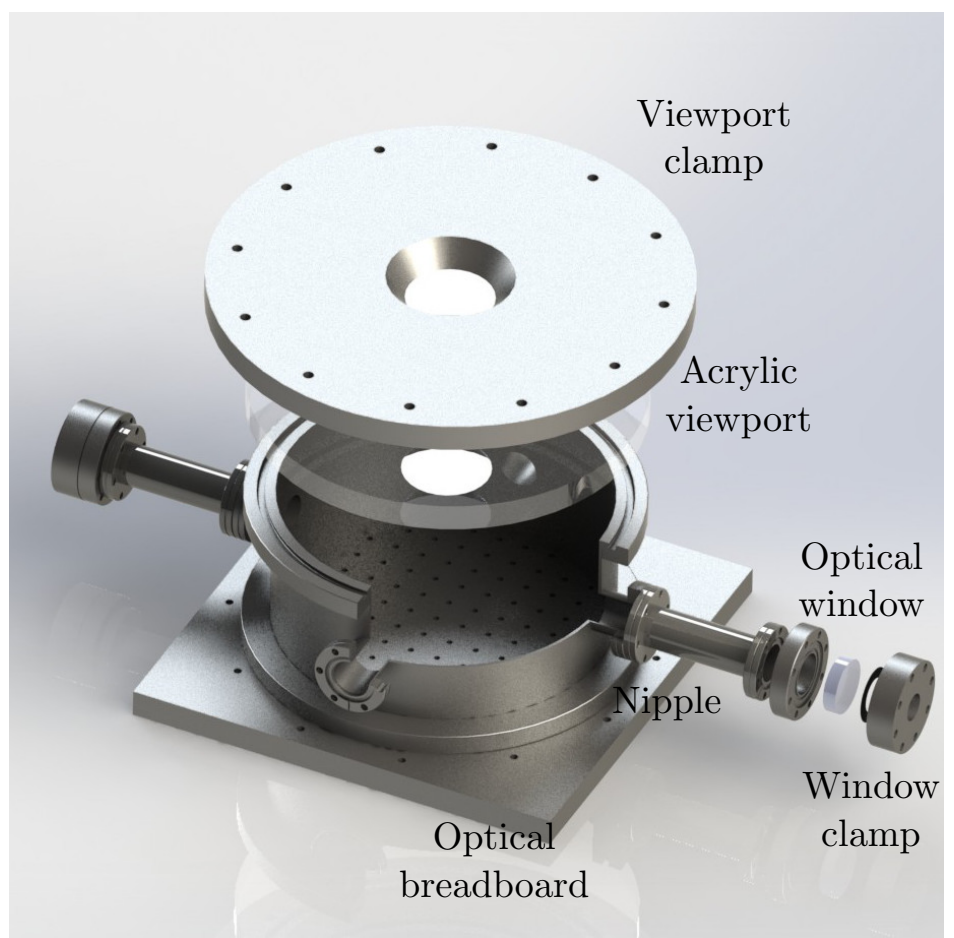

Figure 2.9: Gas chamber for Raman spectroscopy.

The two opposite $2.75^{\prime \prime}$ viewports, through which the centrifuge beam entered the chamber, were additionally extended with vacuum nipples. This was necessary to avoid damage from the high intensity centrifuge beam, focused by a long focal length lens $(f=1 \mathrm{~m})$. The distance between the input and output windows reached $70 \mathrm{~cm}$ after this extension.

The two other ports were used for the vacuum pump, gas line and electrical connections. In the experiments requiring optical probing from a side, they were used to feed through the probe laser beam. The chamber was sealed and evacuated through one of the ports with a hermetic scroll pump down to a few tens of mTorr, and then filled with a studied gas from a gas cylinder.

\subsubsection{Multi-pass amplifier}

Under ambient conditions, when the molecules were initially rotationally hot, an additional amplification of the centrifuge beam was required to adi- 


\subsection{Raman setup}

abatically excite high rotational states. To achieve higher energy, we built a multipass amplifier (MPA). As the gain of a typical Ti:Sapphire amplifier is polarization sensitive, both arms of the centrifuge arms had to be amplified separately prior to being combined into the centrifuge pulse.

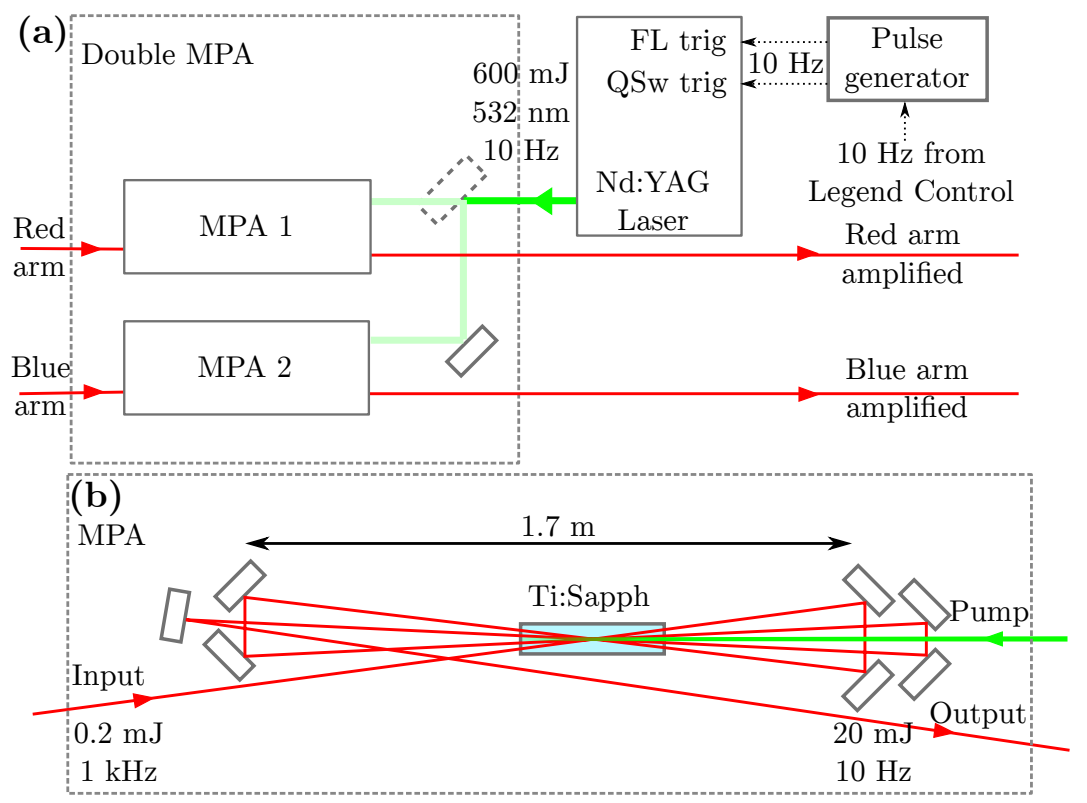

Figure 2.10: (a) Diagram of separate amplification of the two centrifuge beams. (b) Multi-pass amplifier.

The amplifier therefore consisted of two independent MPA's (Fig. 2.10 (a)), pumped by a second harmonic of a Q-switched Nd:YAG laser (Spectra Physics). This laser generated $600 \mathrm{~mJ}$ of $532 \mathrm{~nm}$ light at $10 \mathrm{~Hz}$. A pulse generator (Quantum Composers 9514), synced to every 100th centrifuge pulse, triggered the pump laser's flashlamps (FL) and Q-switch (QSw) at $10 \mathrm{~Hz}$. The output was split on a 50/50 beam splitter, and sent to a pair of identical MPA's, each amplifying its own centrifuge arm.

Inside each MPA, the pump beam was focused down to a few millimeters on a $10 \mathrm{~mm}$ diameter, $35 \mathrm{~mm}$ long Ti:Sapphire crystal rod, used as a gain medium. The crystals were cut at the Brewster's angle to minimize the reflection, and fixed in a water-cooled mounts, to stabilize their temperature at $18^{\circ}$. The input beam, passing through the crystal close to its axis, was retro-reflected by a set of mirrors three times, yielding a total amplification of about 100, from $0.2 \mathrm{~mJ}$ to $20 \mathrm{~mJ}$. 


\subsubsection{Broadband probe beam}
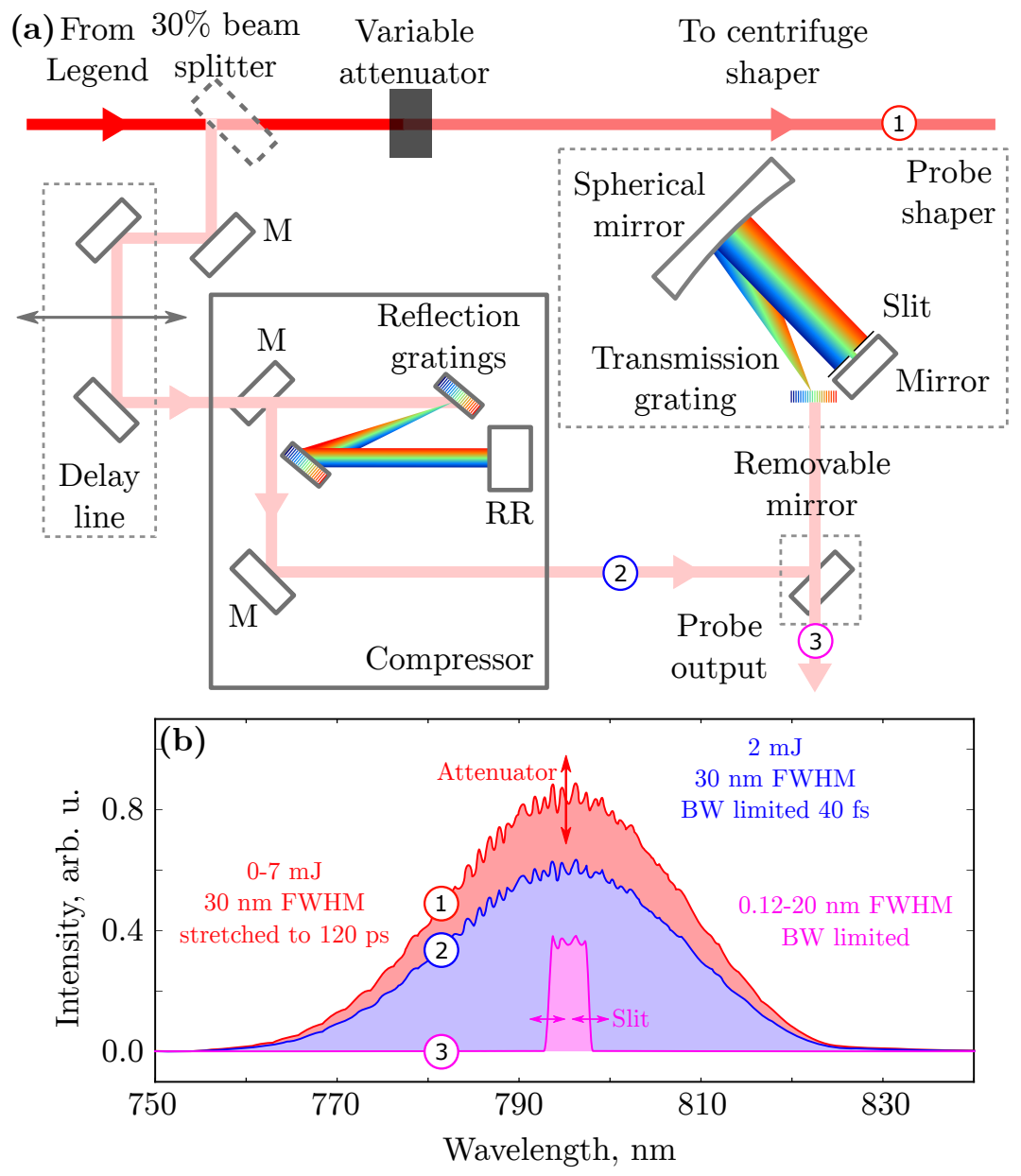

Figure 2.11: (a) Probe beam setup. M: mirror, RR: retroreflector. (b) Full centrifuge spectrum (red), full probe spectrum (blue), and truncated probe spectrum (magenta)

The output of the Legend broadband amplifier was split on a dielectric beam splitter (Layertech, Fig. 2.11), with 30\% (3 mJ) of the total energy serving as a probe and the rest sent to the centrifuge shaper (see subsection 2.1.2). Variable attenuation of the centrifuge beam was provided by the two cube polarisers (Thorlabs PBS-202), with the first one mounted in a manual rotary stage and the second mounted in a fixed holder. Changing 
the angle between the two polarisers allowed us to gradually change the laser energy from $0 \mathrm{~mJ}$ (crossed configuration) to a maximum of $7 \mathrm{~mJ}$ (uncrossed configuration). The more conventional way of attenuating the beam with a rotating wave plate, followed by a polarizer, failed to provide an achromatic attenuation across the bandwidth of our laser pulses, especially at high levels of attenuation.

The probe beam was sent through an optical delay line, computer controlled with a 150-mm travel motorized translation stage (Newport UTS50CC), and compressed in a double-grating compressor (Coherent) to compensate for a chirp introduced in a Legend amplifier. The resulting Fourier transformlimited laser pulse of $40 \mathrm{fs}$ duration was used as a probe in all the experiments requiring high temporal resolution. For the spectrally-resolved detection, on the other hand, the probe was redirected to a standard $4 f$ pulse shaper, where its bandwidth was narrowed with a slit aperture down to $0.12 \mathrm{~nm}$.

\subsubsection{Rotational Raman spectroscopy}

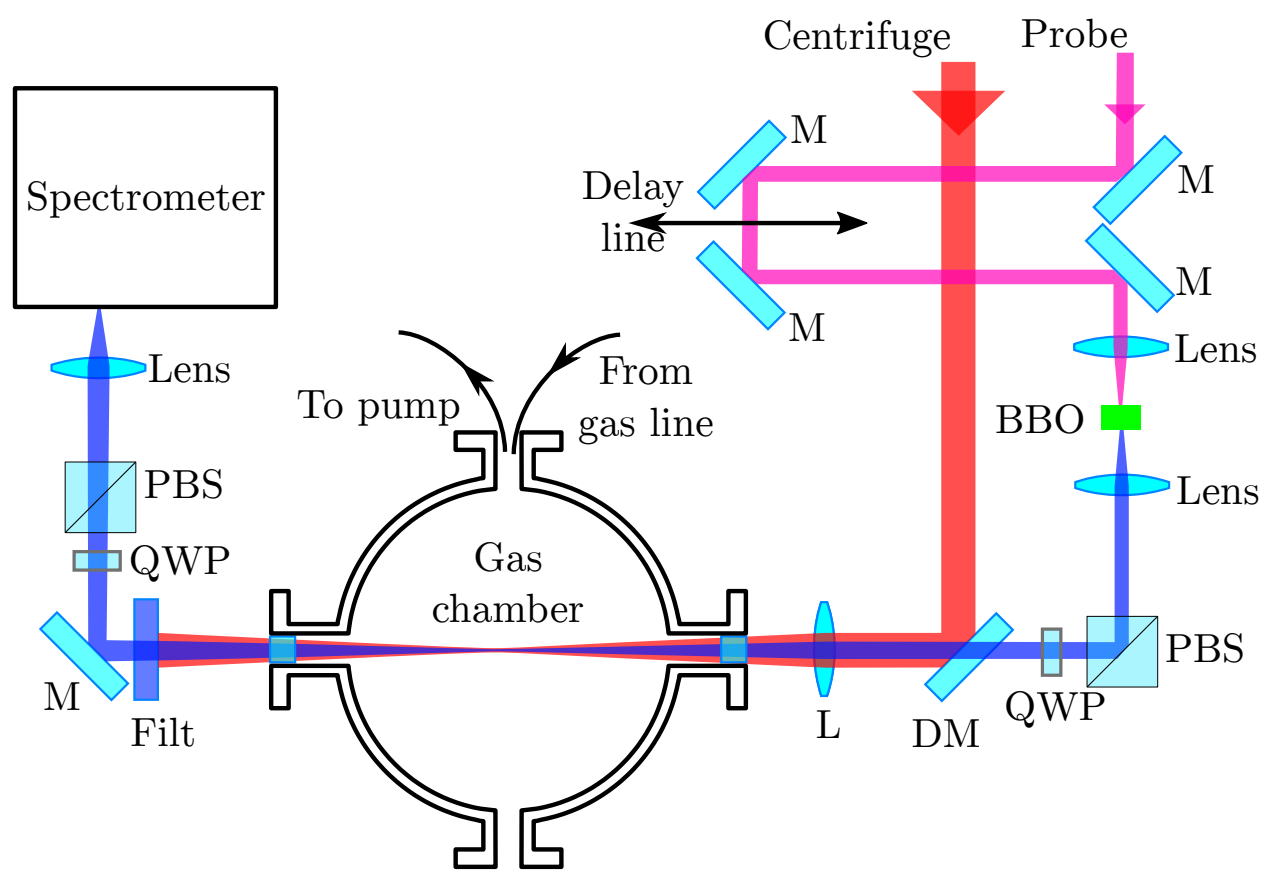

Figure 2.12: Raman setup. PBS: polarizing beam splitter, QWP: quarterwave plate, DM: dichroic mirror, Filt: dichroic filter, L: lens, M: mirrors. 
Probe pulses, circularly polarized with a combination of a polarizer (PBS) and a half-wave plate (HWP) and delayed with a motorized delay line, were scattered off the centrifuged molecules. To separate the weak Raman signal from the strong centrifuge light, probe pulses were frequency doubled to shift their central wavelength to around $400 \mathrm{~nm}$ with total energy of $<1 \mu \mathrm{J} /$ pulse. As shown in Fig. 2.12, the two beams were combined on a dichroic mirror (DM) and focused into the gas chamber with a single $f=1000 \mathrm{~mm}$ lens to a focal beam waist of $\approx 120 \mu \mathrm{m}$ FWHM. Lose focusing was used to avoid ionization and plasma breakdown by limiting the peak intensity of the excitation field to below $5 \times 10^{12} \mathrm{~W} / \mathrm{cm}^{2}$. After the output stage of polarization and wavelength filtering, probe spectrum was recorded with a $0.1 \mathrm{~nm}$-resolution spectrometer (McPherson).

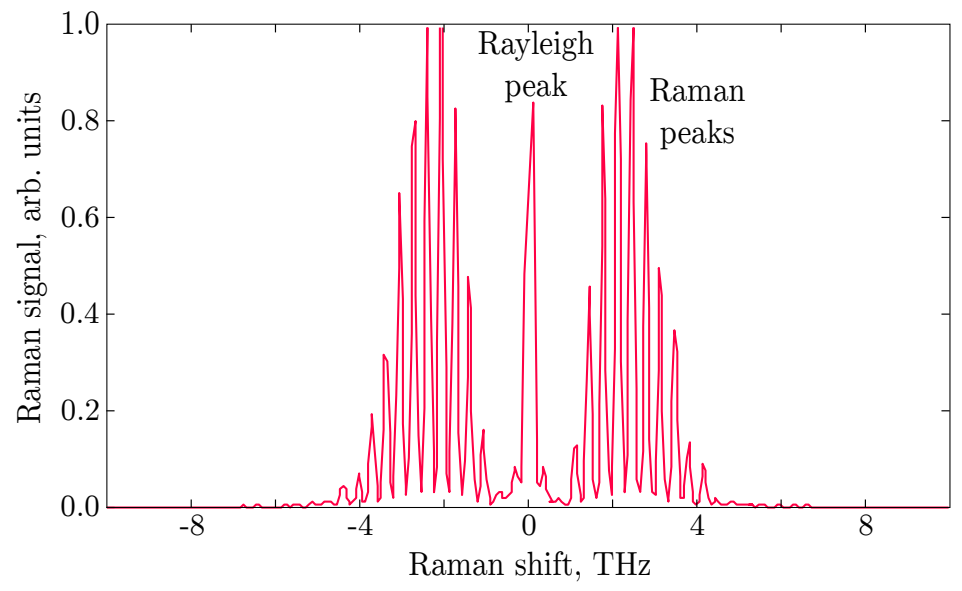

Figure 2.13: Raman spectrum of laser-kicked oxygen.

An example of Raman spectrum of the rotationally excited oxygen gas is shown in Fig. 2.13. The chamber was filled with 0.9 bar of $\mathrm{O}_{2}$, and the molecules were exposed to a single strong femtosecond linearly polarized pulse. This type of excitation, usually referred to as a femtosecond kick, created a broad rotational wave packet in molecules forcing their axes to rotate towards the laser polarization. The fs kick was followed by a $0.375 \mathrm{~cm}^{-1}(0.1$ $\mathrm{THz}$ ) wide probe. The figure shows the observed spectrum of the probe light scattered from the kicked molecules. It consists of a single Rayleigh peak at the zero frequency shift, which stems from the laser-induced permanent molecular alignment. The latter leads to a non-zero optical birefringence and the corresponding change in the probe polarization. 
Around the central line, there is a series of narrow Raman peaks. Each peak to the right corresponds to anti-Stokes Raman scattering $|N\rangle \rightarrow$ $|N+2\rangle$, while each peak to the left - to Stokes Raman scattering $|N+2\rangle \rightarrow$ $|N\rangle$. The $N$-th peak has a detuning $\epsilon_{N+2}-\epsilon_{N}=B(N+2)(N+3)-$ $B N(N+1)=2 B(2 N+3)$. Since $N$ in oxygen takes only odd positive values [47, the distance between the two consecutive peaks is expected to be $8 B=11.4 \mathrm{~cm}^{-1}=0.34 \mathrm{THz}$ in a good agreement with the observation. Since equal amount of molecules are rotating both clockwise and counterclockwise in the kicked ensemble, the Stokes and anti-Stokes peaks are of similar height.

\subsection{Resonance-enhanced multiphoton ionization spectroscopy}

Rotational Raman spectroscopy which works well in dense ensembles becomes inapplicable in a cold ultrasonic jet, where the gas density is 8 orders of magnitude lower. To detect the low density products, resonance-enhanced multiphoton ionization (REMPI) is often employed due to its high sensitivity, spectral resolution and versatility [2, 81].

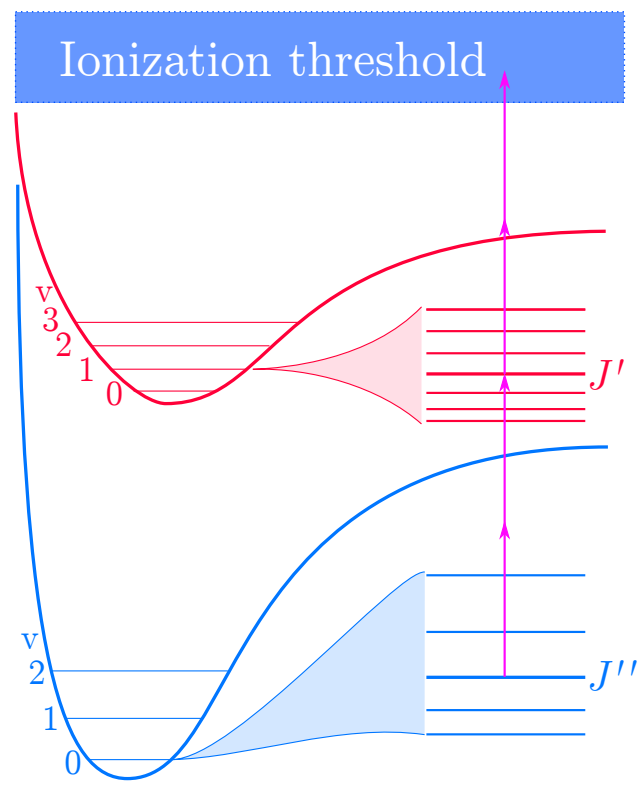

Figure 2.14: REMPI principle. 
The principle of REMPI spectroscopy is depicted in Fig. 2.14. Consider a molecule in some rotational state $\left|n^{\prime \prime}=0, v^{\prime \prime}, J^{\prime \prime}\right\rangle$ of the ground electronic state, where $n^{\prime \prime}$ and $v^{\prime \prime}$ are the principal and the vibrational quantum numbers. Using a narrowband ultra-violet laser, the molecule can be resonantly excited via a one- or a few-photon transition to another ro-vibrational state $\left|n^{\prime}=1, v^{\prime}, J^{\prime}\right\rangle$. Since these states are much closer to the ionization threshold, they can be ionized by absorbing one or more photons from the same laser field. Measuring the ion current as a function of the laser wavelength, one detects peaks each time the wavelength matches the multiphoton resonance $\left|n^{\prime}, v^{\prime}, J^{\prime}\right\rangle \leftarrow \leftarrow \cdots \leftarrow\left|n^{\prime \prime}, v^{\prime \prime}, J^{\prime \prime}\right\rangle^{3}$. The strength of each peak is proportional to the population of the initial state $\left|n^{\prime \prime}, v^{\prime \prime}, J^{\prime \prime}\right\rangle$ and the probability of this multiphoton transition. Knowing the latter one can extract the molecular rotational distribution.

\subsubsection{Utra-high vacuum chamber and molecular source}

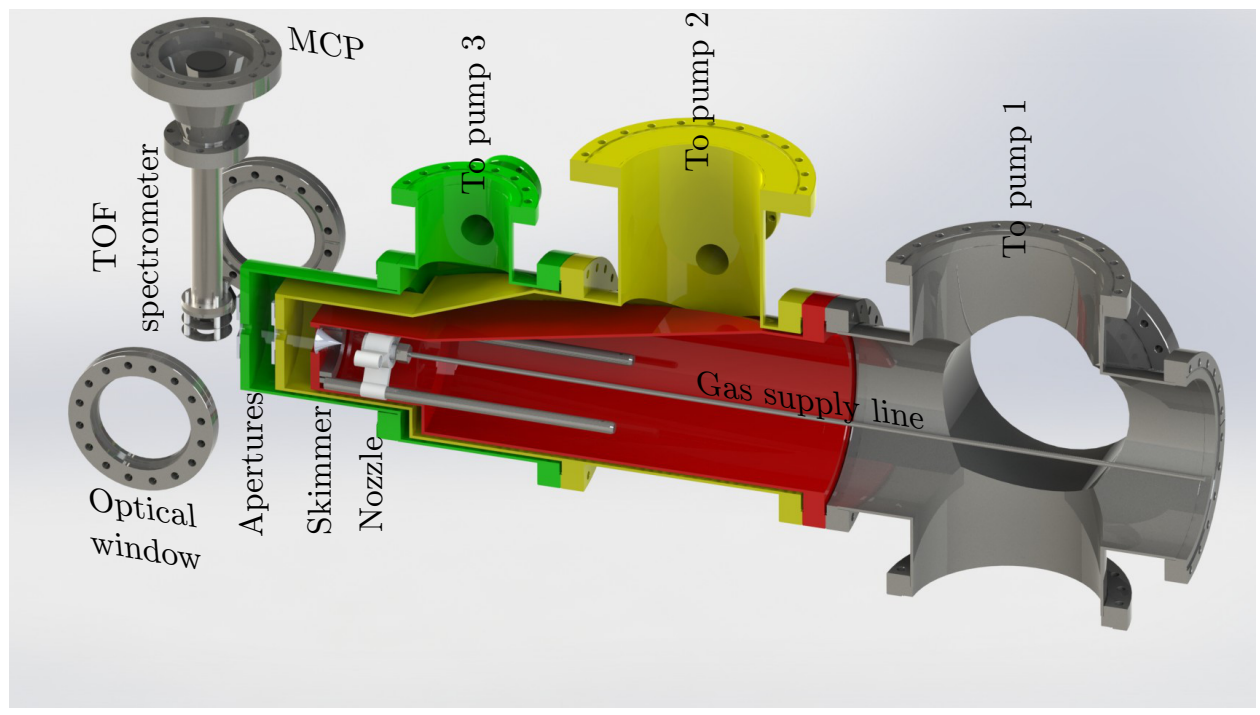

Figure 2.15: Ultra-high vacuum chamber with a molecular source.

High signal-to-noise ratio spectroscopy of molecular super rotors requires

\footnotetext{
${ }^{3}$ Here we follow the standard spectroscopic convention, where the ground state is placed on the right hand side, the excited - on the left hand side, and the number of arrows correspond to the number of absorbed photons. The ground end excited states quantum numbers are marked with two and a single primes, respectively
} 
a dense source of rotationally cold molecules. This is commonly achieved with a supersonic expansion of gas from a high pressure, of order of tens of bars, to low pressure, usually below $10^{-4}$ Torr through a small nozzle[108]. In practice, maintaining this pressure difference in a continuous mode of operation requires unrealistic pumping speeds, hence the nozzles are often equipped with pulsed valves, working in sync with the laser pulses. In our work, we used Even-Lavie valve with a nozzle diameter of $150 \mu \mathrm{m}$, capable of producing sub-20 $\mu$ s gas bursts with a back-pressure of up to 100 bar and a repetition rate of up to $1 \mathrm{kHz}$.

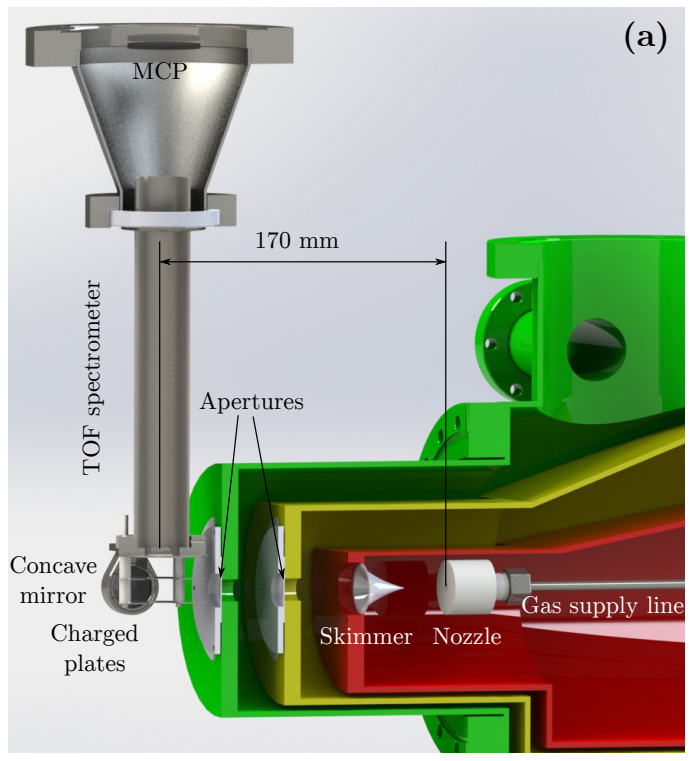

(b)
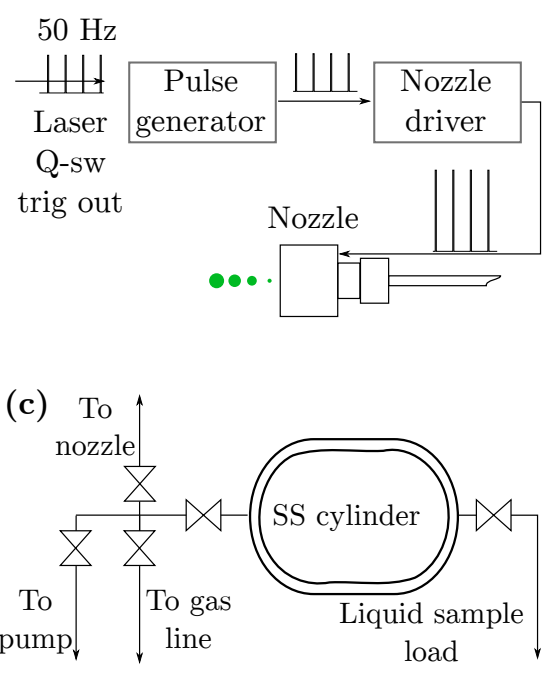

Figure 2.16: (a) Magnified view of the UHV chamber. (b) Nozzle control electronics. (c) Gas line connections diagram.

Even with a duty cycle this low, the pressure in the vacuum chamber reached $10^{-4}$ Torr, which was too high for the detection scheme based on molecular ionization. To overcome this, a custom design UHV chamber (Nor-Cal Products, Inc.) was built (Fig. 2.15 (a)). It consisted of four differentially pumped sections, connected via small cross-section apertures aligned along the trajectory of the expanding gas. The first section (red in Fig. 2.15 (a)) contained the nozzle, while the measurements were carried out in the last "science" chamber. To lessen the effects of shock waves, the first aperture was a $1.5 \mathrm{~mm}$ nickel skimmer (Beam Dynamics, Inc., 
see Fig. 2.16 (a)). As the amount of gas in the other two chambers was considerably smaller, simple stainless steel plates with $2 \mathrm{~mm}$ holes were used there.

Since the gas density in the beam drops rapidly $\left(\sim \frac{1}{r^{2}}\right)$ with the distance from the nozzle, we tried to keep the latter as close to the detection region as possible. This was achieved by nesting the three vacuum sections inside one another and allowed us to have the nozzle only $170 \mathrm{~mm}$ away from the detection region.

All four sections were pumped with turbomolecular pumps (Leybold (1000 l/s), KYKY (1300 l/s), Pfeiffer (260 l/s), Edwards (75 l/s)). These pumping speeds enabled us to pump out the science chamber below $3 \times$ $10^{-8}$ Torr at the highest used backing pressures of 30 bar and repetition rates of $1 \mathrm{kHz}$.

The nozzle valve was driven by a pulsed current driver (Even-Lavie), triggered externally by a signal from a pulse generator (BNC 555), synced to the output of the Nd:YAG laser trigger (Fig. 2.16 (b)). The pulse generator was employed for synchronizing the laser pulses with the gas bursts, as it took a few hundreds of microseconds, depending on the gas mixture, for the molecules to reach the detection region. For pure oxygen, this time was about $\sim 320 \mu \mathrm{s}$, which means the trigger timing had to be advanced by this amount (in practice, it was achieved by delaying it by $T_{\text {rep }}-320 \mu \mathrm{s}$, where $T_{\text {rep }}$ is a period of the Nd:YAG pulses).

The connection diagram of a high-pressure gas line is shown in Fig. 2.16 (c). Using a few splitters and valves allowed us to connect the nozzle directly to the gas supply, or to the stainless steel cylinder, prefilled with the required gas mixture. The whole system was connected to a hermetic vacuum pump (Agilent IDP-15) for the safe removal of potentially hazardous gas species.

\subsubsection{Time-of-flight spectrometer}

In a low-density molecular jet environment very high sensitivity experimental techniques are required. Modern avalanche-based ion detectors, such as micro-channel plate detectors (MCP), are extremely sensitive, as they are capable of detecting single ions. For this reason, all methods used in the UHV chamber were based on photoionization by either a fs or a ns probe laser pulse, followed by the ion collection.

To detect the ions, a time-of-flight spectrometer was used (Fig. 2.17). The ions were extracted from the interaction region and directed towards a dual microchannel plate detector (MCP, Tectra MCP-050-D-P46-V) with a DC field, created by a set of high voltage electrodes. The MCP detector was 


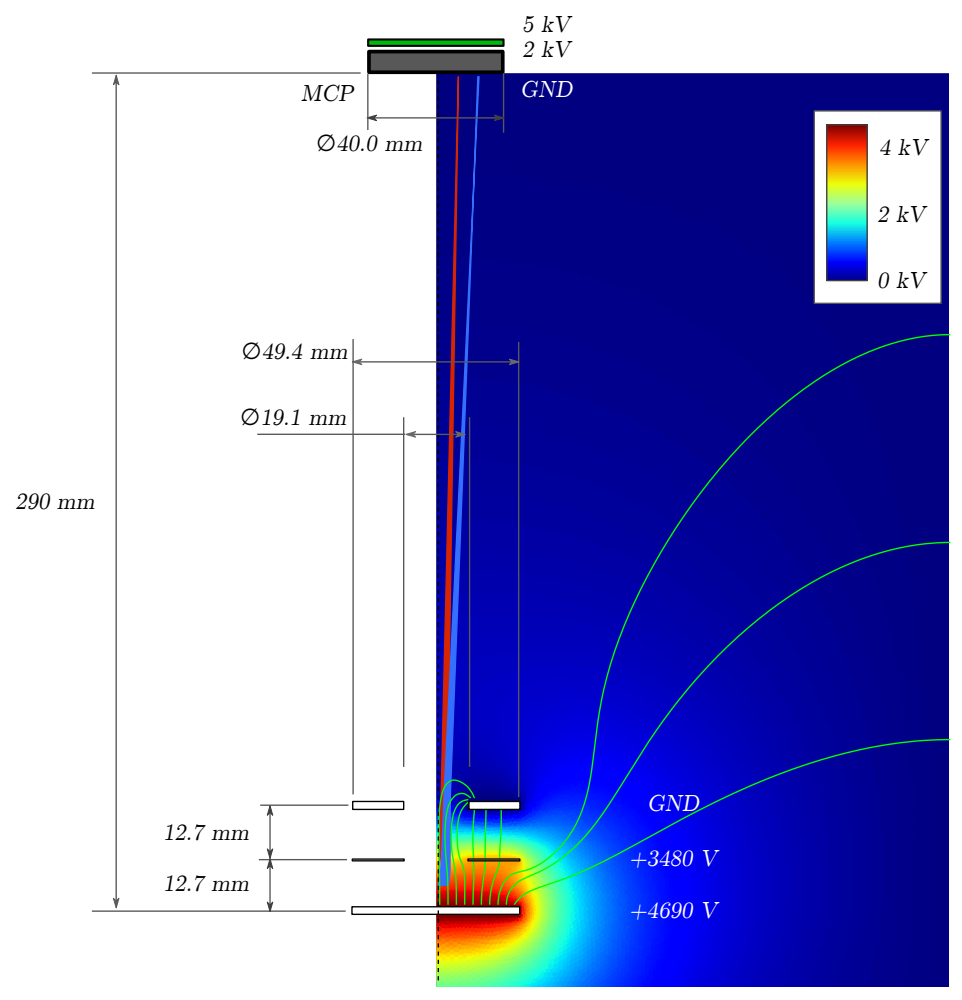

Figure 2.17: Geometry of the time-of-flight spectrometer (left) and the simulated electric field distribution (right).

equipped with a fast phosphor screen, mounted next to an optical viewport. A single ion collision with the detector gave rise to an electronic avalanche, that, further accelerated towards the phosphor screen, produced a bright spot on the screen. The time of ion arrival was determined by the ion's velocity acquired from the electric field, which in turn depended on the particle mass-to-charge ratio. These times were of order of a few microseconds for the voltages used. As the phosphorescence lifetime for our phosphor was $55 \mathrm{~ns}$, it was usually possible to distinguish between the ions of different species by the timing of the corresponding luminescent peaks. Although the mapping function can be calculated numerically, in practice, a calibration was required to achieve a reasonable accuracy.

The three disk-shaped electrodes with circular holes were made out of stainless steel and held together by UHV-compatible ceramic insulators (Kimball Physics). When the voltages on the plates were adjusted for proper 


\subsection{Resonance-enhanced multiphoton ionization spectroscopy}

ion focusing, the point at which an ion would hit the MCP was determined solely by its horizontal velocity. We carried out the numerical calculations in Comsol software (Fig. 2.17, right half) to find the optimal electrode voltages. For REMPI, where only a light electron leaves the molecule, its velocity changes insignificantly. Since the molecules in the beam are translationally cold, all of the produced ions would have the same initial velocity (the speed of the supersonic expansion), giving a single spot close to the center of the screen.

The light from the phosphor screen was collected with an aspheric condenser lens $(\mathrm{f}=40 \mathrm{~mm})$ and focussed onto either a photo-multiplier tube (PMT, Hamamatsu H10721-01), sensitive to the phosphor emission peak at $520 \mathrm{~nm}$, or a low-noise fast CCD camera (Point Grey Grasshopper 3).
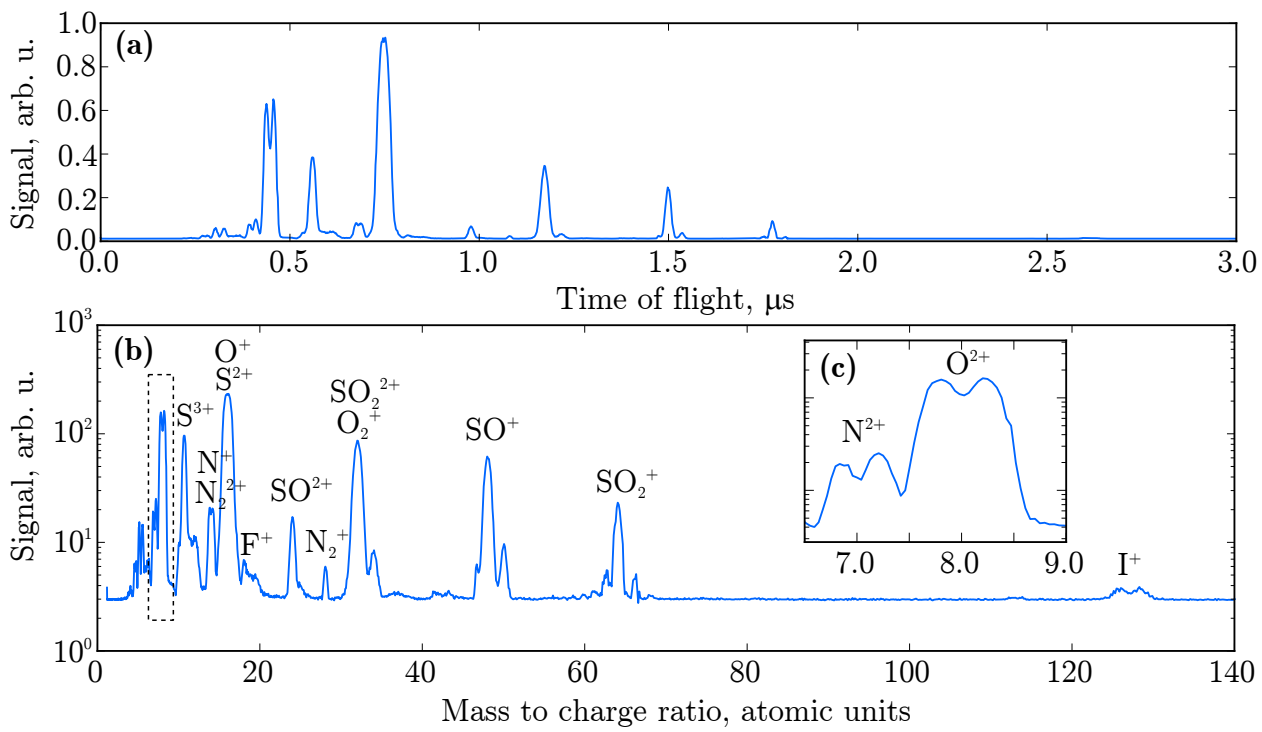

Figure 2.18: (a) Raw time-of-flight spectrum of photo-dissociated mixture of gases. (b) Calibrated spectrum. (c) Magnified region of the spectrum around mass-to-charge ratio of 8 .

MCP detector was equipped with a gate (Photek GM-MCP), capable of switching the high voltage from $1.5 \mathrm{kV}$ to $2.0 \mathrm{kV}$ and back with a $20 \mathrm{~ns}$ time constant. Since the detector is relying on a highly non-linear process of avalanche formation, the amplification levels at these two input voltages were different by several orders of magnitude. By timing the gate with respect to the photoionization laser, we could probe only the ions arriving 
within a 20 ns time window.

A mix of several substances $\left(\mathrm{O}_{2}, \mathrm{~N}_{2}, \mathrm{SO}_{2}, \mathrm{C}_{6} \mathrm{H}_{3} \mathrm{IF}_{2}\right)$ was introduced into the chamber and exposed to a 35-fs intense laser pulse. The pulse caused the molecules to fragment into ions of various masses and charges. Measuring the total signal (either on camera or on PMT) as a function of the gate delay, we obtained the spectrum shown in Fig. 2.18 (a). In this plot, peaks correspond to the arrival times of the ions with different mass-to-charge ratios.

The velocity, acquired by a resting ion of mass $m$ and charge $q$, when it crosses the potential difference $V$ is $v=\sqrt{\frac{2 q V}{m}}$. The time of reaching the detector is therefore $t=C \sqrt{\frac{m}{q}}$, where $C$ is the calibration constant. We tuned $C$ so that all the observed peaks corresponded to the mass-to-charge ratios of the expected ion fragments from our gas mixture (Fig. 2.18 (b)). Other ions were then readily identified.

As can be clearly seen in Fig. 2.18 (c), some of the peaks demonstrated a peculiar doublet structure, symmetric around the expected arrival time of the corresponding ions. The underlying effect, known as Coulomb explosion, was crucial for using the velocity map imaging technique for the reconstruction of the molecular angular distribution, discussed in section 2.4.

\subsubsection{Dye laser system}

The laser setup used for REMPI spectroscopy is shown in Fig. 2.19. A 50 $\mathrm{Hz}$ Nd:YAG laser with a frequency doubler (Spectra Physics Quanta Ray) generated nanosecond pulses carrying $250 \mathrm{~mJ}$ at $532 \mathrm{~nm}$, which were used to pump a three-stage dye laser (Sirah Cobra-Stretch). The active medium for the latter was prepared by dissolving Rhodamine $6 \mathrm{G}$ in ethanol with a concentration of $0.09 \mathrm{~g} / \mathrm{l}$ for the resonator and preamplifier stages and $0.01125 \mathrm{~g} / 1$ for the amplifier. The output of the dye laser was frequency doubled in the second harmonic generator system (Sirah SHG-250), yielding $5 \mathrm{~mJ}$ per pulse tunable from $279 \mathrm{~nm}$ to $288 \mathrm{~nm}$. Computer control of the laser wavelength was carried out by simultaneous adjustment of the resonator and the second harmonic generation BBO angle, to reach the optimal phase matching.

To synchronize the fs and ns pulses, we took a $1 \mathrm{kHz}$ trigger signal from the Legend control unit and divided its frequency 20-fold, bringing it down to $50 \mathrm{~Hz}$, in a pulse generator (Quantum Composers 9514). The two outputs of the generator were used as the triggers for the Nd:YAG laser's flashlamps and Q-switch. The flashlamps trigger was additionally advanced by $130 \mu \mathrm{s}$ 


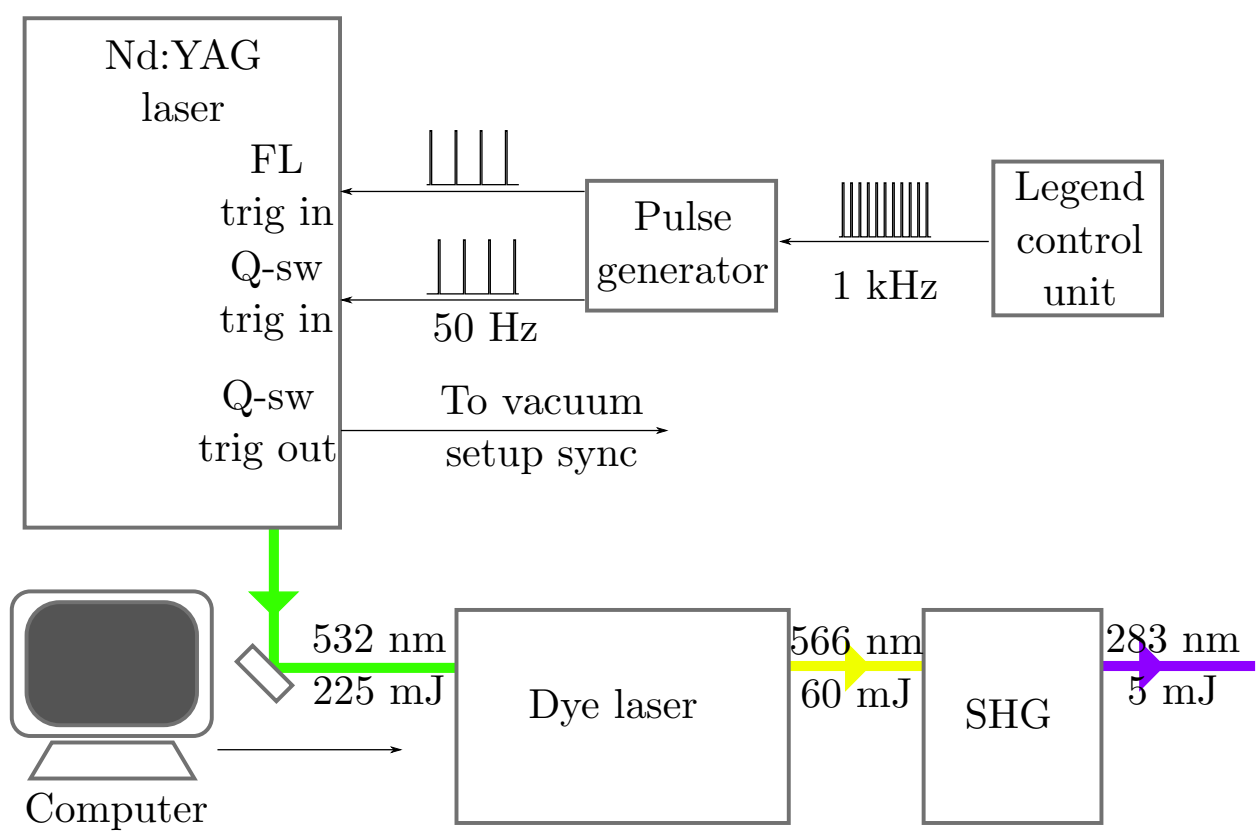

Figure 2.19: Dye laser system. The flashlamps (FL) and Q-switch (Q$\mathrm{sw})$ of Nd:YAG pump laser were synchronized with the centrifuge pulses electronically. The output of the dye laser was frequency doubled in a second harmonic generation system (SHG).

to achieve an optimal population inversion prior to the Q-switch firing.

\subsubsection{REMPI spectroscopy of $\mathrm{N}_{2}$ ant $\mathrm{O}_{2}$}

Experimental setup for REMPI spectroscopy is shown in Fig. 2.20. Nanosecond probe pulses were combined with a centrifuge beam by means of a dichroic mirror. Oxygen or nitrogen at a pressure of 20 bar was supplied to the back of the nozzle.

The blue line in Fig. 2.21 shows the measured $(2+2) a^{1} \Pi_{g}\left(v^{\prime}=0\right) \leftarrow \leftarrow$ $X^{1} \Sigma_{g}^{+}\left(v^{\prime \prime}=1\right)$ REMPI spectrum of nitrogen. Here, " $2+2$ " means that it takes two photons of a probing laser to excite a molecule from its initial ground electronic and vibrational state $\Sigma_{g}^{+}\left(v^{\prime \prime}=0\right)$ to an excited electronic state $a^{1} \Pi_{g}\left(v^{\prime}=1\right)$, and then another two photons to ionize it.

Since each photon bears an angular momentum of 1 , an absorption of two photons can change the molecular angular momentum by not more than 


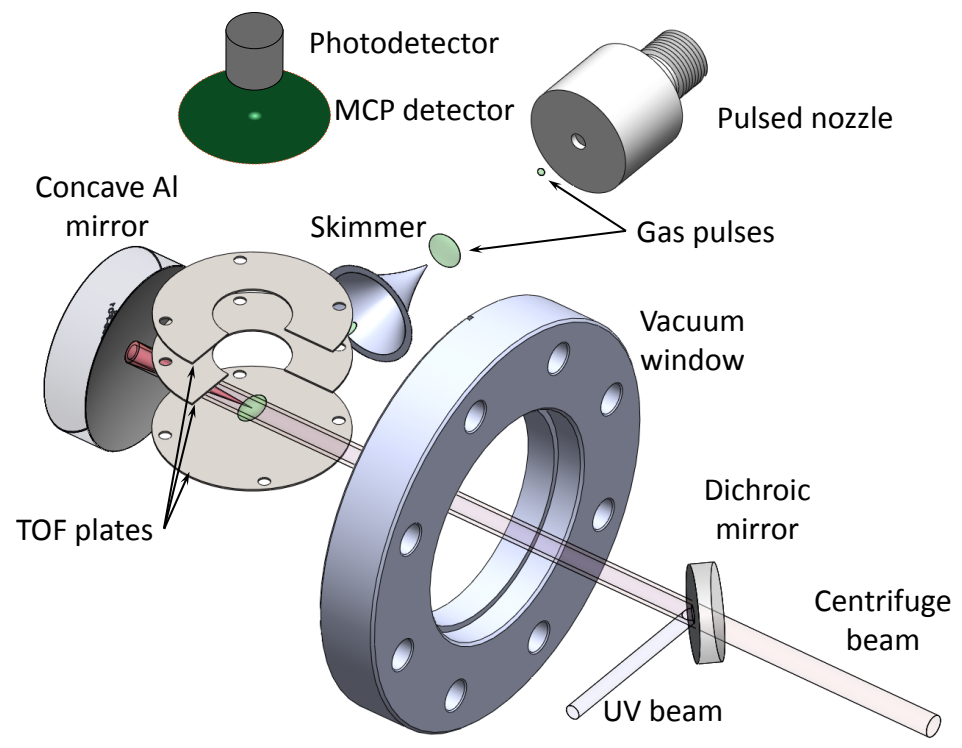

Figure 2.20: REMPI spectroscopy setup. Centrifuge beam is combined with a tunable UV laser pulse and focused inside a vacuum chamber on a supersonically expanded molecular jet between the charged plates of a timeof-flight (TOF) mass spectrometer. The ionization rate is measured with a multi-channel plate (MCP).

2. It is natural to classify the two photon transitions by the change of the total angular momentum $\Delta J=J^{\prime}-J^{\prime \prime}$. The possible changes are: $\Delta J=2$ (S-branch), $\Delta J=1$ (R-branch), $\Delta J=0$ (Q-branch), $\Delta J=-1$ (P-branch) and $\Delta J=-2$ (O-branch). The transition energies of these branches are shown in Fig. 2.21 by ticks on the black lines, with $J^{\prime \prime}$ changing by one from one tick to the next.

In the absence of the electronic spin, the rotational structure of both the initial and the final states correspond to a rotational structure of a simple symmetric rotor and is easy to calculate. The largest peak on the blue line could then be identified as a transition from $J^{\prime \prime}=0$ of S-branch, etc. After identifying the peaks and accounting for the line strength, we could determine the initial population of all rotational states and estimate the rotational temperature to be around $10 \mathrm{~K}$.

In the case of nitrogen, the identification of REMPI peaks is simple even at higher temperatures. For example, the red curve in Fig. 2.21 shows a REMPI spectrum of nitrogen at $300 \mathrm{~K}$. For this experiment, the gas was 


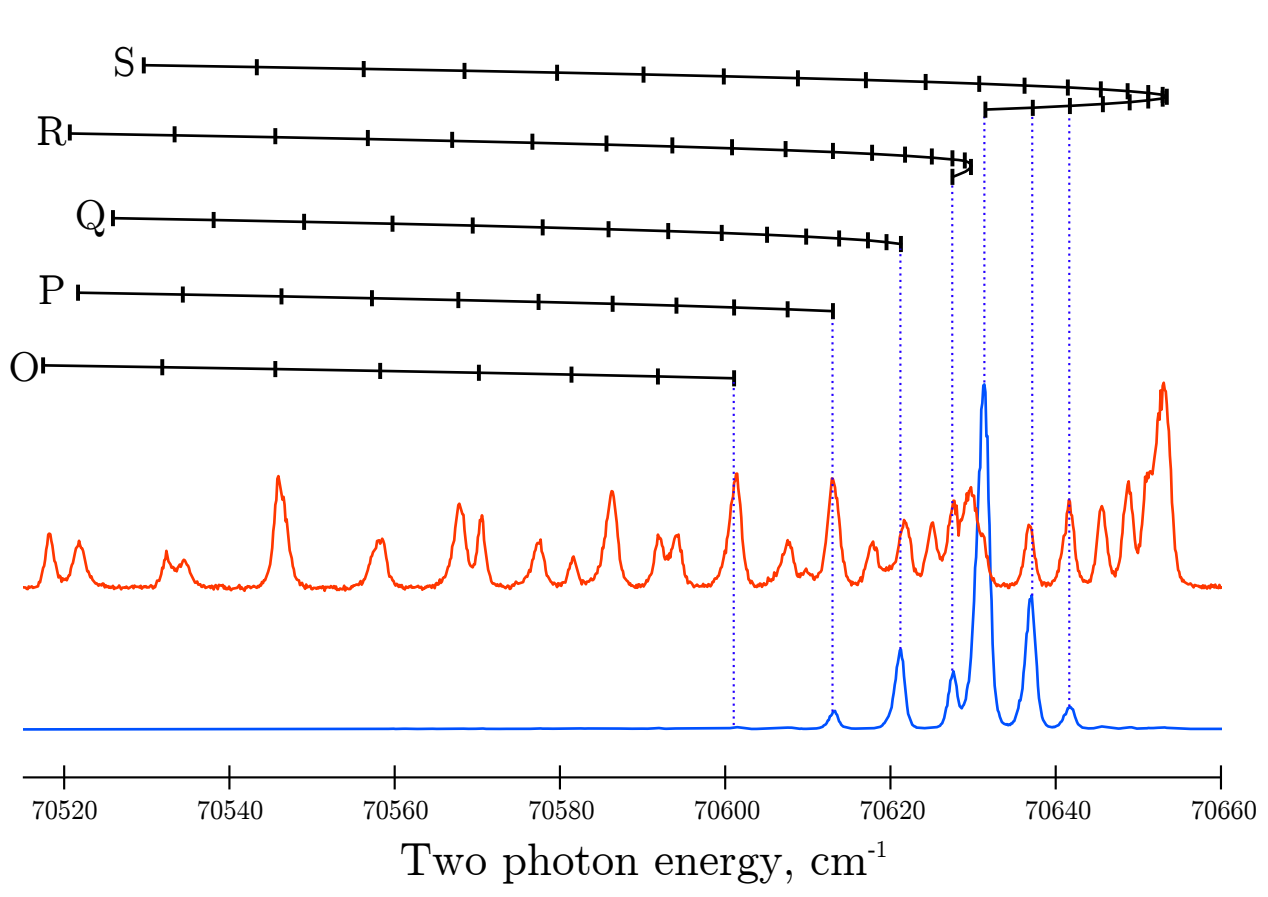

Figure 2.21: REMPI spectra of cold ( $T=10 \mathrm{~K}$, blue) and room temperature (red) nitrogen. Black ticks, connected by lines to guide the eye, show the calculated transition energies for different branches.

leaked in the chamber through the leak valve. Even though the number of resonances is very high, almost each one of them could be separated and attributed to a particular transition, which makes the determination of rotational population still possible.

In oxygen, $(2+1) C^{3} \Pi_{g}\left(v^{\prime}=2\right) \leftarrow \leftarrow X^{3} \Sigma_{g}^{-}\left(v^{\prime \prime}=0\right)$ REMPI is often employed due to the convenience of the laser wavelength, the high ionization cross-section, and rotationally resolved structure. The line identification is, however, substantially more complicated compared to nitrogen. First, the intermediate state $C^{3} \Pi_{g}\left(v^{\prime}=2\right)$ pre-dissociates, which leads to significant line broadening of about $10 \mathrm{~cm}^{-1}$. Second, both the ground and excited states have a non-zero spin, which leads to a more complicated energy level structure (Fig. 2.23). In the ground state, oxygen molecule has a zero electron orbital momentum projection $\Lambda$ on the internuclear axis, and the energy structure follows Hund's case (b) [11]. The energy states are determined by the angular momentum excluding electronic spin, that takes 


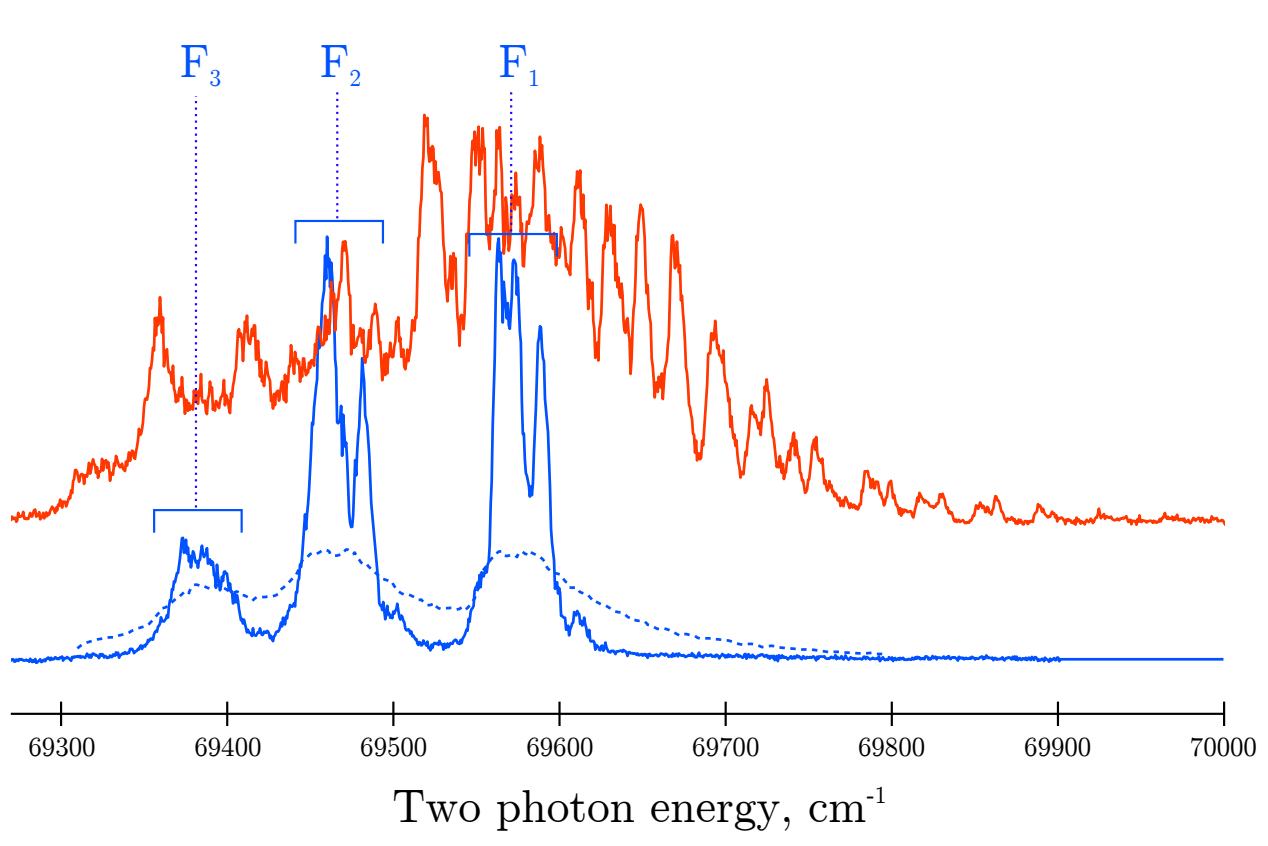

Figure 2.22: REMPI spectra of cold ( $T=10 \mathrm{~K}$, blue) and room temperature (red) oxygen. Dashed line demonstrates the effect of power broadening.

values $N^{\prime \prime}=1,3, \ldots$ For each $N^{\prime \prime}$ there is a triplet of states corresponding to the total angular momentum $J^{\prime \prime}=N^{\prime \prime}-1, N^{\prime \prime}, N^{\prime \prime}+1$. The line splitting in the triplet is due to the spin-spin and spin-rotation interaction. The excited state has $\Lambda=1$ and, therefore, follows Hund's case (a). It consists of three different sets of rotational levels, according to the total electron angular momentum projection $\Omega^{\prime}=0,1,2$. For each $\Omega^{\prime}$ the levels are determined by their total angular momentum $J^{\prime}=\Omega^{\prime}, \Omega^{\prime}+1, \ldots$

Due to the small value of spin splitting in the ground state (less, than 1 $\mathrm{cm}^{-} 1[47]$ ), it was not resolved in our experiments. It is, therefore, useful to designate the transition branches by two numbers $\Omega^{\prime}$ and $\Delta N=J^{\prime}+\Omega^{\prime}-$ $N^{\prime \prime}-1\left[122\right.$. For each value of $\Omega^{\prime}$, there are 7 possible $\Delta N$, giving a total of 21 branches. As a result, when the number of excited rotational levels in the ground state increases, the spectrum becomes very congested, as can be seen in Fig. 2.22, where the blue and the red lines correspond to $10 \mathrm{~K}$ and room temperature ensembles, respectively. 


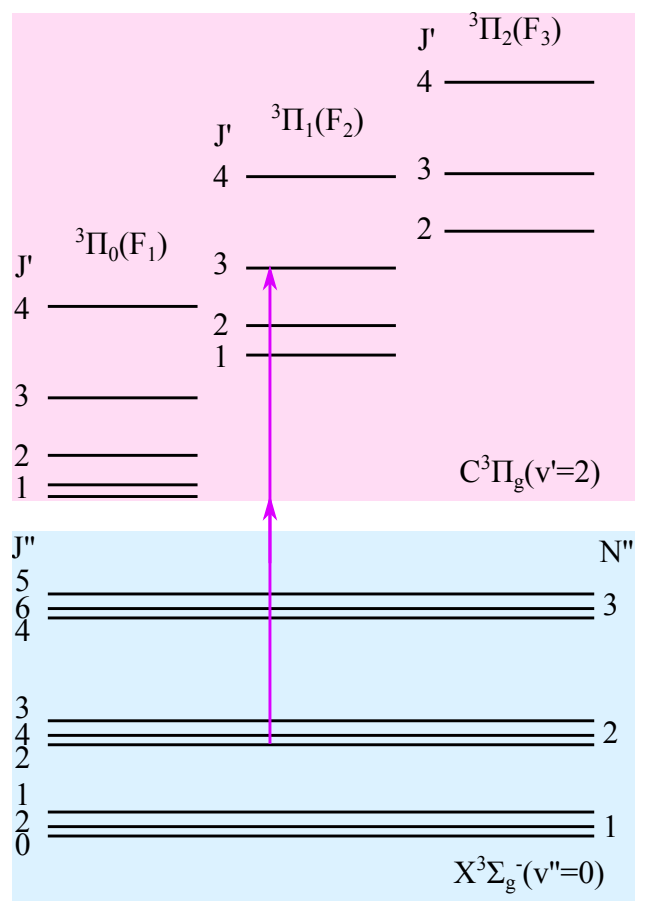

Figure 2.23: Illustration of the $C^{3} \Pi_{g}\left(v^{\prime}=2\right) \leftarrow \leftarrow X^{3} \Sigma_{g}^{-}\left(v^{\prime \prime}=0\right)$ transition in oxygen. Blue (red) area marks the rotational structure of the ground (excited) electronic state, affected by the spin-spin and spin-rotational (spinorbit) interaction.

\subsection{Velocity-map imaging (VMI)}

When a molecule is placed in a non-resonant laser field of high intensity $\left(>10^{14} \mathrm{~W} / \mathrm{cm}^{2}\right)$ it often undergoes a process of multiphoton ionization [34], leaving a highly charged molecular ion. This cation is often unstable and the smaller ions which it consists of, experience strong Coulomb repulsion. In a process, called Coulomb explosion, they immediately recoil from each other, releasing large amount of kinetic energy.

The direction of the recoil is correlated with the angular coordinates of the molecule. If the laser pulse used for the Coulomb explosion is short enough, so that the molecule being ionized cannot rotate during the explosion [27, 34, 100], the fragment momenta can be used to measure the molecular angular distribution right before the ionization [27]. For example, diatomic molecules, such as nitrogen, recoil in the direction of their 

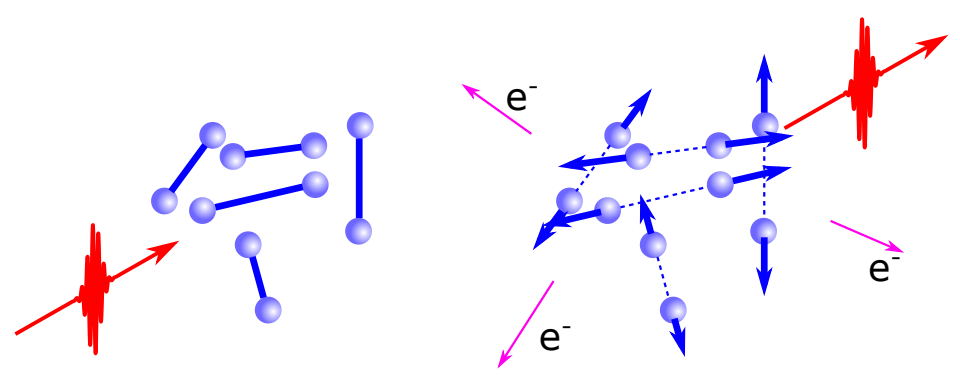

Figure 2.24: Illustration of the process of Coulomb explosion. After being ionized by a fs pulse, the molecules immediately fragment into ions recoiling in the direction of their respective internuclear axes.

internuclear axis, as demonstrated in Fig. 2.24. Measuring the full fragment velocity distribution would therefore allow one to extract the molecular angular distribution.

The kinetic energy released in the Coulumb explosion is strongly dependent on the channel of dissociation. For example, for the $\mathrm{N}_{2}^{3+} \rightarrow \mathrm{N}^{2+}+\mathrm{N}^{+}$ channel, the ions recoil with the energy around $15 \mathrm{eV}$ and a distribution width of $5 \mathrm{eV}$, while for $\mathrm{N}_{2}^{4+} \rightarrow \mathrm{N}^{2+}+\mathrm{N}^{2+}$ the distribution is centered around $29 \mathrm{eV}$ and has a width of $10 \mathrm{eV}[25$.

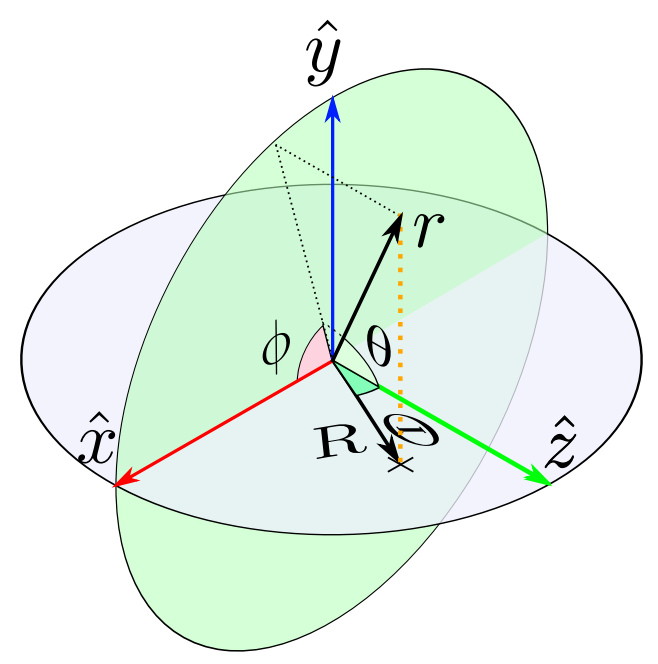

Figure 2.25: Relevant polar variables for the velocity map imaging. 
Advanced techniques, such as cold target recoil-ion momentum spectroscopy (COLTRIMS), allow to determine the full three-dimensional momentum distribution of ions[126]. Our VMI setup was suitable for measuring the projection of the fragments momentum onto the plane of the MCP. Let us consider a fragment with an initial velocity $\mathbf{v}$, and introduce vector $\mathbf{r}=\mathbf{v} t$, where $t$ is the time of reaching the detector. Vector $\mathbf{R}$, defining the position in the MCP detector plane, where this ion would be observed, is then a projection of $\mathbf{r}$ onto a detector's plane.

It is useful to introduce a coordinate system $x y z$, with $x z$ plane corresponding to the plane of the detector, spherical coordinates $\mathbf{r}=(r, \theta, \phi)$ and polar coordinates $\mathbf{R}=(R, \Theta)$ in the $x z$ plane, as defined in Fig. 2.25 . Knowing the fragment velocity distribution $f(r, \theta, \phi)$ we can find the ion image distribution $F(R, \Theta)$ via [128]:

$$
F(R, \Theta)=\int_{R}^{\infty} \frac{r f(r, \theta, \phi)}{\sqrt{r^{2}-R^{2}}} d r
$$

with $\theta=\arccos \left(\frac{R \cos \Theta}{r}\right)$ and $\phi= \pm \arccos \frac{\tan \Theta}{\tan \theta}$. This so-called Abel transform is just an integral along the line normal to the detector (dashed orange line in Fig. 2.25) of all $\mathbf{r}$ contributing to the same $\mathbf{R}$.

In practice, the task is usually the opposite - to find the velocity distribution $f(\mathbf{r})$ knowing the measured ion image $F(\mathbf{R})$. Although its not possible in general, because $\mathbf{r} \rightarrow \mathbf{R}$ correspondence is not unique, methods implying an additional symmetry from $f(\mathbf{r})[23,26,38,132$. or using several projections along different directions [118] were used to successfully find the inverse.

The most popular class of Abel transform inversion methods uses the basis of $2 \mathrm{D}$ images with known $3 \mathrm{D}$ distributions to expand the experimental ion images and reconstruct the underlying recoil velocity distribution. This approach was first proposed by Dribinski and co-workers, who suggested using a basis of Gaussian functions [26]. This method, which the authors called BASEX, proved inaccurate for our images, predicting unphysical divergences along the axis of symmetry. Its modification, utilizing a more natural basis of Legendre polynomials for the angular dependencies, known as pBASEX method [38], was computationally hard because of the large number of the basis functions required for the case of high rotational harmonics. The algorithm that worked best for us belonged to the family of Maximum Entropy Methods (MEM), often employed to reconstruct noisy data and adapted to the analysis of velocity maps by Dick [23], who kindly shared his software with us. The idea of the method is to find the velocity 
distribution which gives a projection close to the measured one, but doesn't assume any new information (or, in other words, has the highest entropy among the other distributions with similar projections). Since it is only applicable to the distributions with an axial symmetry, which wasn't the case in many interesting scenarios, and since the reconstruction suffered from the strong drift effect (see section 3.3), it was not used in our analysis. In section 5.1, we describe an approximate method we used for estimating the degree of molecular alignment from the acquired VMI data.
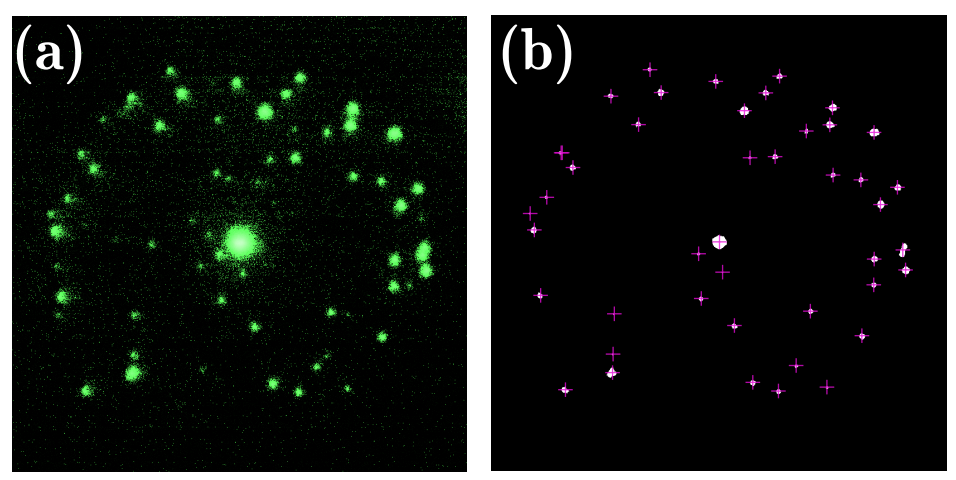

Figure 2.26: (a) Raw ion image after a single laser shot. (b) Processed image with the determined ion positions, marked by the magenta crosses.

\subsubsection{Velocity map imaging reconstruction of the angular distribution}

Having calibrated a TOF spectrometer, we were able to isolate a particular type of ions we were interested in. For example, after setting our gate to the mass-to-charge ratio of 14, a single femtosecond laser shot resulted in an image shown in Fig. 2.26 (a). Each small spot on the image corresponds to a single collision of an atomic $\mathrm{N}^{+}$ion with a phosphor screen. The ions are scattered around the center due to the kinetic energy they acquired in a Coulomb explosion of randomly oriented cold nitrogen molecules. The bright spot in the center corresponds to molecular ions $\mathrm{N}_{2}^{2+}$. Zero kinetic energy release (since the two removed electrons are too light to considerably change the momentum of the molecule) results in the narrow distribution width.

To extract the angular distributions from such images, we had to average these images over many laser shots. One method, involving a low 
frame rate camera, was to take long exposure pictures, with each pixel of the camera registering multiple ions. The disadvantage of this method was its requirement of high homogeneity of the MCP amplification across its area. For this reason, we later moved to a fast camera, working at repetition rates from a few hundred $\mathrm{Hz}$ to a $\mathrm{kHz}$. Using an algorithm written in Python programming language and utilizing OpenCV computer vision library, the images were acquired from the camera in real time, noise filtered, threshold-discriminated, and the ion peak positions were determined (Fig. 2.26 (b)). After accumulating a million ion peaks, we plotted a 2D histogram, corresponding to the final $F(\mathbf{R})$ distribution (Fig. 2.27).
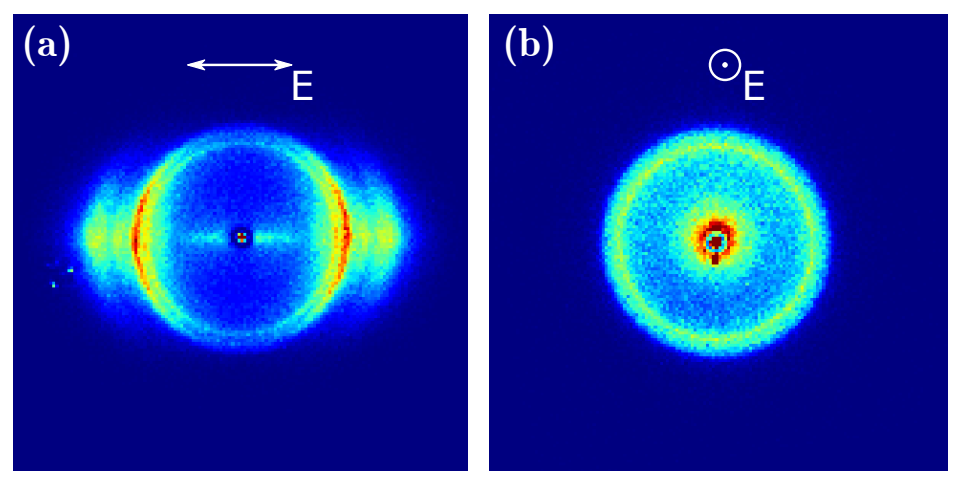

Figure 2.27: $\mathrm{N}^{+}$ion image distribution measured for 1,000,000 events, taken with probe polarization (a)parallel and (b)normal to the detector plane.

The two images in Fig. 2.27 (a) and (b) were taken with a probe linearly polarized parallel to the plane of the detector and perpendicularly to it, respectively. Picture (a) has a clear circle structure, with each circle corresponding to the largest $R$ for a given dissociation channel. As was mentioned above, since each channel has a kinetic energy release narrowly distributed around its average energy, these circles come mostly from the molecules aligned in the detector plane at the moment of explosion.

Another important feature of Fig. 2.27 (a) is its high anisotropy with respect to $\Theta$. An isotropic molecular angular distribution is expected to give rise to a centrally symmetric ion image, while the anisotropic one corresponds to an aligned ensemble. This apparent alignment along the direction of the probe polarization could be accounted by two different effects [100]. First, the so-called geometric alignment comes from the anisotropy of the ionization cross sections - molecules which happened to be aligned along 
the laser polarization direction are more likely to be ionized and explode in the direction of their instantaneous alignment. The second effect, called the dynamic alignment, corresponds to the molecules being aligned by the Coulomb explosion field itself.

While the geometric alignment can be accounted for in molecular angular distributions calculations by a simple normalization, the dynamic alignment, typically observed in light molecules such as $\mathrm{H}_{2}$ and $\mathrm{N}_{2}[100]$ complicates these calculations as it affects the angular distributions. Fortunately, the effect of the dynamic alignment can be reduced by polarizing the probe pulses circularly or lowering their intensity [27, 93].

\subsubsection{Centrifuge angle measurement}

During the shaping, the two centrifuge arms traveled separately over a distance of a few meters. Mirrors vibration and air movement in the lab lead to the randomization of their relative optical phase, changing from pulse to pulse. Owing to this random phase, the centrifuge "corkscrew" envelop was randomly rotated along the propagation axis in each laser shot. As a result, the molecules were released at a random angle $\theta_{\text {rel }}$ with respect to horizontal axis (see Fig. 2.28), but with the same angular frequency. Hence, the angular distribution of super rotors, determined by the method described in the previous section, was averaged over the rotations along the beam propagation direction.

For a number of experiments, we needed to know the value of $\theta_{\text {rel }}$ for each centrifuge pulse or, alternatively, the angle of centrifuge polarization $\theta(\tau)$ at any fixed delay $\tau$ from the rising edge of the centrifuge.

As was mentioned above (section 2.1.2), overlapping the centrifuge and a short reference pulse on a doubling crystal allowed us to measure the cross-correlation signal, proportional to the centrifuge field intensity as a function of time. Due to the polarization sensitivity of the sum-frequency generation, this signal reflects the strength of a single projection of the centrifuge field onto the axis of the BBO crystal. If we assume that the angle of the centrifuge polarization changed in time as $\beta \tau^{2}+\theta_{0}$, where $\tau$ is the time measured from the centrifuge rising edge, $\beta$ is the frequency chirp, and $\theta_{0}$ is the (random) initial polarization angle, then the cross correlation signal would be proportional to $\cos ^{2}\left(\beta \tau^{2}+\theta_{0}\right)$ when the reference pulse is delayed by $\tau$.

Using $5 \%$ beam splitters we picked off a small portion of the centrifuge and the femtosecond probe beams to use them for the angle measurement. Both beams were split in two equal parts on a 50/50 beam splitter and 


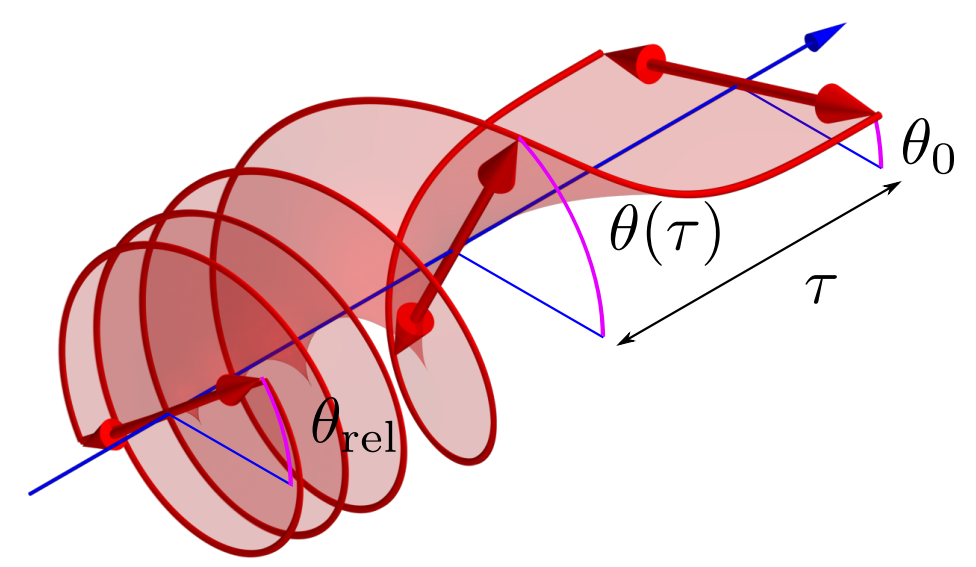

Figure 2.28: Definition of the centrifuge angles.

focused on a pair of identical BBO crystals $(30 \mu \mathrm{m}$ Newlight Photonics, Fig. 2.29 (a)). A $1 \mathrm{~mm}$ glass plate was inserted in the centrifuge path in one of the arms, to slightly delay it relative to the reference beam. The measured cross correlation signals behind the first and the second crystals were therefore proportional to $\cos ^{2}\left(\beta\left(\tau+\tau_{\text {glass }}\right)^{2}+\theta_{0}\right)$ and $\cos ^{2}\left(\beta \tau^{2}+\theta_{0}\right)$, respectively, where $\tau_{\text {glass }}=\frac{d}{c}\left(n_{\text {glass }}-1\right)$ is an additional delay, acquired by the centrifuge in the first arm due to the glass plate, $d=1 \mathrm{~mm}$ is the plate thickness, $n_{\text {glass }}$ is the refractive index of the glass and $c$ is the speed of light.

The correlation plot of the two signals measured over many laser shots reproduced a Lissajous figure of a ratio one, with a phase equal to $4 \beta \tau \tau_{\text {glass }}+$ $2 \beta \tau_{\text {glass }}^{2}$, as demonstrated in Fig. 2.29 (b), where different colours correspond to different values of the delay $\tau$. With enough points accumulated, we were able to fit an ellipse to them, and extract the centrifuge angle $\theta_{\text {rel }}$ for every laser shot from the angular position of a point on this ellipse corresponding to that shot. To achieve the best precision of this extraction, $\tau$ was tuned to obtain circular correlation plot (red circles on Fig. 2.29 (b)). 

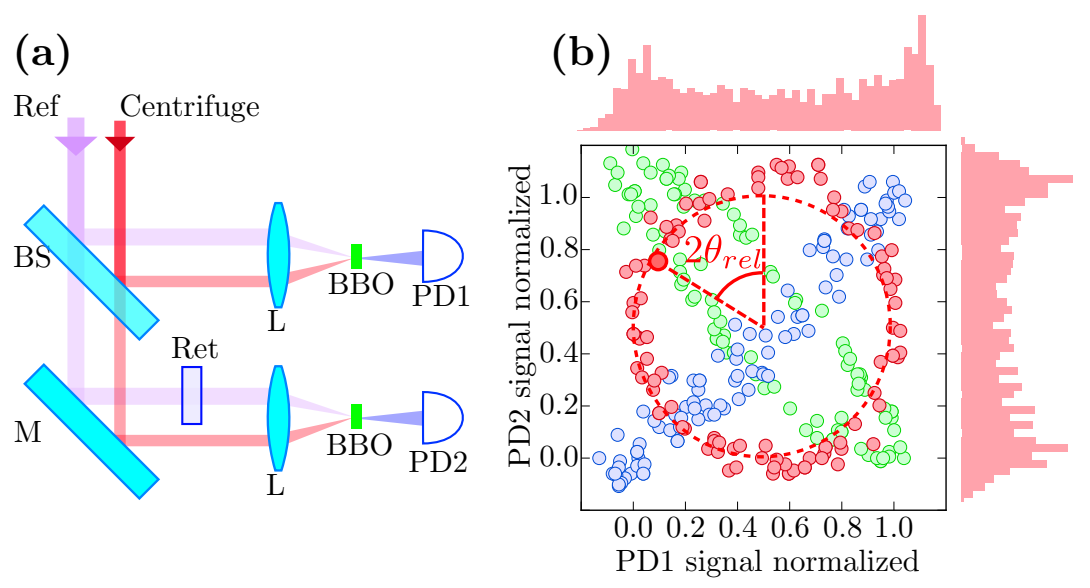

Figure 2.29: (a) Angle measurement setup. Ref: reference femtosecond laser pulse, BS: 50/50 beam splitter, L: lenses, Ret: glass plate retarder, PD1, PD2: photodiodes. (b) Correlation plot of the two normalized signals from PD1 and PD2, acquired over 100 laser shots. Red bar charts show the histograms of the two signals. Scatter plots of different colors correspond to three different delays between the reference and the centrifuge pulses, with the red trace corresponding to an optimal delay, used for determining $\theta_{\text {rel }}$. 


\section{Chapter 3}

\section{Direct detection of molecular super rotors}

Since the original proposal[57, an optical centrifuge has been implemented by two experimental groups. In the pioneering work by Villeneuve et al., dissociation of chlorine molecules exposed to the centrifuge field has been attributed to the breaking of the $\mathrm{Cl}-\mathrm{Cl}$ bond which could not withstand the extremely high spinning rates[130]. More recently, Yuan et al. observed rotational and translational heating in the ensembles of $\mathrm{CO}_{2}$ and $\mathrm{N}_{2} \mathrm{O}$ molecules and associated it with the collisional relaxation of the centrifuged species [138, 139]. In both cases, an incoherent secondary process (i.e. dissociation and multiple collisions) has been used for indirect identification of the formation of super rotors whose most unique property their synchronous uni-directional rotation, remained hidden. In this chapter, we describe the three detection techniques, that we used to directly detect molecular super rotors.

The first section of this chapter (section 3.1) describes the application of coherent Raman spectroscopy to detect extreme rotational states in a dense molecular gas. This method allowed us to see the field-enforced coherent adiabatic excitation inside an optical centrifuge to rotational frequencies of up to $10 \mathrm{THz}$, followed by a field-free rotation of molecules, and to demonstrate the flexibility of controlling the directionality and frequency of the induced rotation.

Rotationally resolved resonance-enhanced multiphoton ionization (REMPI) spectroscopy is a very sensitive method, capable of detecting extremely low gas densities. The interpretation of REMPI spectra in the case of rotationally hot molecules is not always straightforward, as it relies on the exact knowledge of the intermediate electronic state. The controllability of the centrifuge excitation was used in a novel spectroscopy method and allowed us to interpret the congested REMPI spectra of oxygen (section 2.3), complicated by the spin-rotational and spin-orbit splittings and pre-dissociation of the excited electronic state.

The two methods mentioned above were employed to probe the energy 
spectrum of the rotational states. A different approach was developed to probe the molecular instantaneous angular distributions. In the last section (section 3.3) we discuss the use of the velocity-map imaging technique to visualize the angular distributions and their dynamics, modified by the ultrafast rotation of molecules.

\subsection{Raman spectroscopy of molecular super rotors}

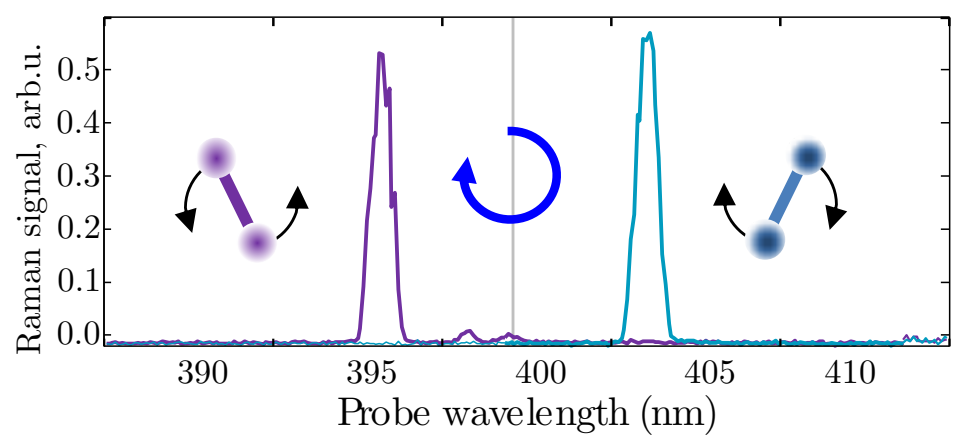

Figure 3.1: Probe spectra after passing through the counter-clockwise (purple) and clockwise (cyan) centrifuged oxygen molecules. The appearance of only anti-Stokes or only Stokes sidebands indicate unidirectional rotation.

To carry out the Raman spectroscopy of super rotors we used the setup discussed in detail in section 2.2. We excited ensembles of various diatomic gases $\left(\mathrm{O}_{2}, \mathrm{~N}_{2}, \mathrm{H}_{2}\right)$ with an optical centrifuge in a dense room temperature gas sample and probed it with pulses, spectrally narrowed to $0.375 \mathrm{~cm}^{-1}$ and centered around $400 \mathrm{~nm}$ with a total energy of $<1 \mu \mathrm{J} /$ pulse.

As the probe light passed through the ensemble of the centrifuged molecules, its spectrum developed sidebands with a detuning magnitude equal to twice the molecular angular frequency Fig. 3.1. Choosing the centrifuge to be either counter- or co-rotating with the probe polarization, we observed the appearance of only anti-Stokes (purple) or only Stokes (cyan) sideband. This proves the unidirectionality of molecular rotation. Notice that in both cases, the sidebands were circularly polarized, oppositely to the initial probe polarization, as expected from the selection rules. Indeed, the centrifuge is inducing coherence between the states of the rotational ladder with $\Delta N=2$ and $\Delta M_{N}=2$. To change the angular momentum projection by exactly two 
in the Raman scattering process, the absorbed and emitted photons should bear the angular momentum projection of 1 and -1 , respectively. This corresponds to the fields, circularly polarized in the opposite directions. Hence, using a circular polarizer allowed us to almost completely suppress the much stronger initial probe line.
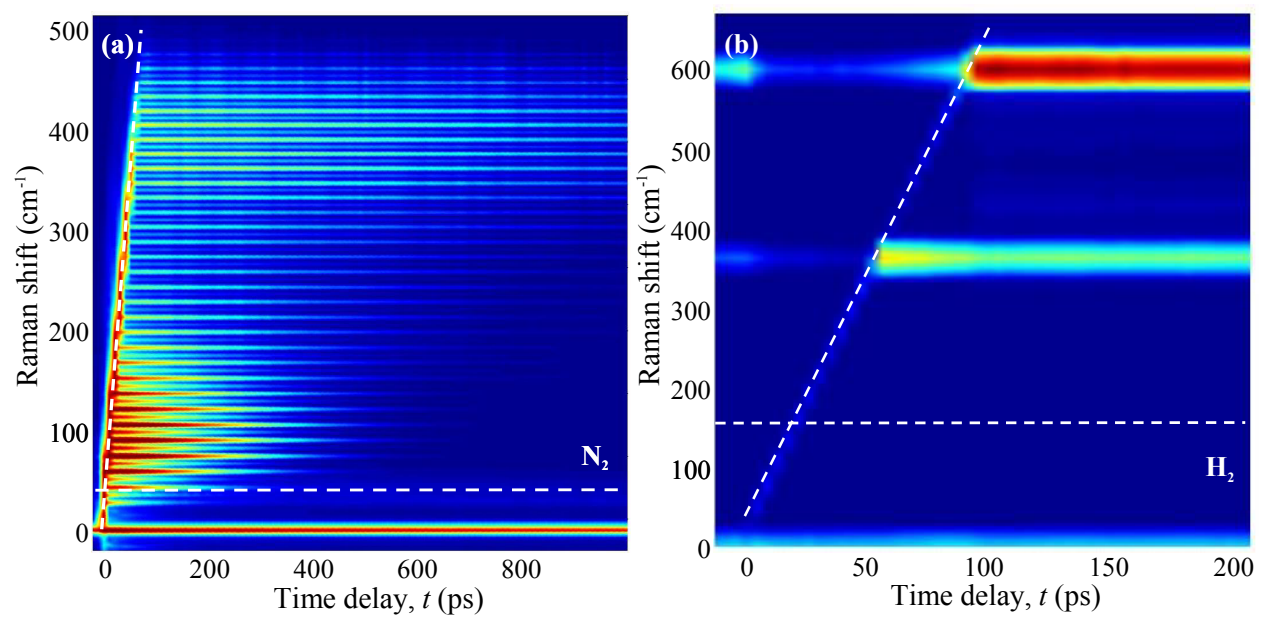

Figure 3.2: Time-dependent Raman shifts from the centrifuged $\mathrm{N}_{2}(\mathbf{a})$ and $\mathrm{H}_{2}$ (b) molecules. As the molecules spend longer time in the centrifuge, the observed Raman frequency shift increases along the slopped dashed lines, providing a direct evidence of accelerated molecular rotation. Some of the molecules "leak" from the accelerating angular trap, leaving the horizontal traces at the intermediate Raman shifts. Horizontal dashed lines correspond to the angular velocity of rotational states, mostly populated at room temperature.

Delaying the arrival time of probe pulses with respect to the beginning of the centrifuge pulse enabled us to observe the spinning molecules before and after they leave the centrifuge. In Fig. $3.2(\mathbf{a}, \mathbf{b})$, the rotational Raman spectrum of nitrogen and hydrogen is plotted as a function of time the molecules spent in the centrifuge. The tilted dashed white line on Fig. 3.2 (a) marks the accelerated spinning of the molecules inside the centrifuge. As their angular frequency grows linearly with time, so does the Raman shift. While spinning up, the molecules are "leaking out", producing a set of Raman sidebands, with each one corresponding to free molecular rotation with quantized angular velocities. Similar behavior was observed in hydrogen 
Fig. 3.2 (b). Due to its small moment of inertia, the level separation is around 30 times larger than in $\mathrm{N}_{2}$ and, as a result, we were only able to populate a few rotational states.

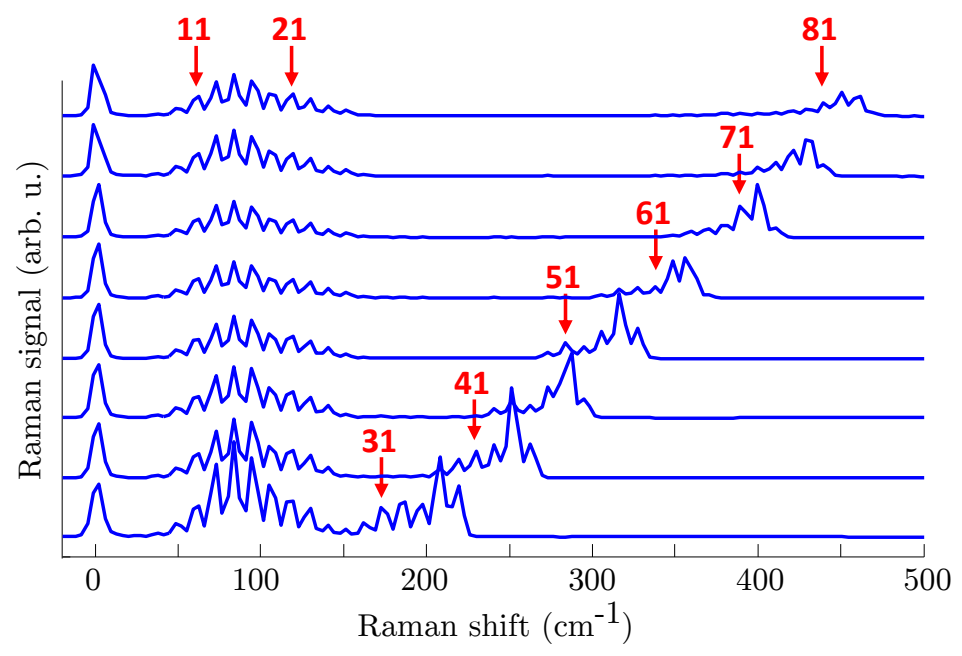

Figure 3.3: State-resolved Raman spectra of centrifuged oxygen molecules. Higher curves correspond to longer spinning time inside the centrifuge. Red vertical arrows mark the rotational quantum numbers.

To identify the excited rotational levels, we analyzed the Raman spectrum of super rotors. The results, corresponding to different centrifuge durations and, therefore, different degrees of rotational excitation, are shown in Fig. 3.3 for the case of $\mathrm{O}_{2}$. Each measured spectrum consists of three parts: (i) an unshifted probe line at $0 \mathrm{~cm}^{-1}$; (ii) a set of stationary lines centered around $80 \mathrm{~cm}^{-1}$ and corresponding to the molecules lost from the centrifuge during its spinning; and (iii) a set of moving lines corresponding to the molecules which followed the centrifuge up to its terminal angular frequency.

Well resolved peaks in the spectrum correspond to the individual Raman transitions between the states with rotational quantum numbers $N-2$ and $N$. The discreetness of the observed spectra follows from the angular momentum quantization. The group of the Raman-shifted lines on the right side of the spectra in Fig. 3.3 reflects an accelerated rotation of the molecules trapped in the centrifuge field, and demonstrates our ability to control the degree of rotational excitation. Quantum numbers $N$ can be easily assigned by counting the peaks. Only odd values of $N$ are allowed for ${ }^{16} \mathrm{O}_{2}$ molecule 


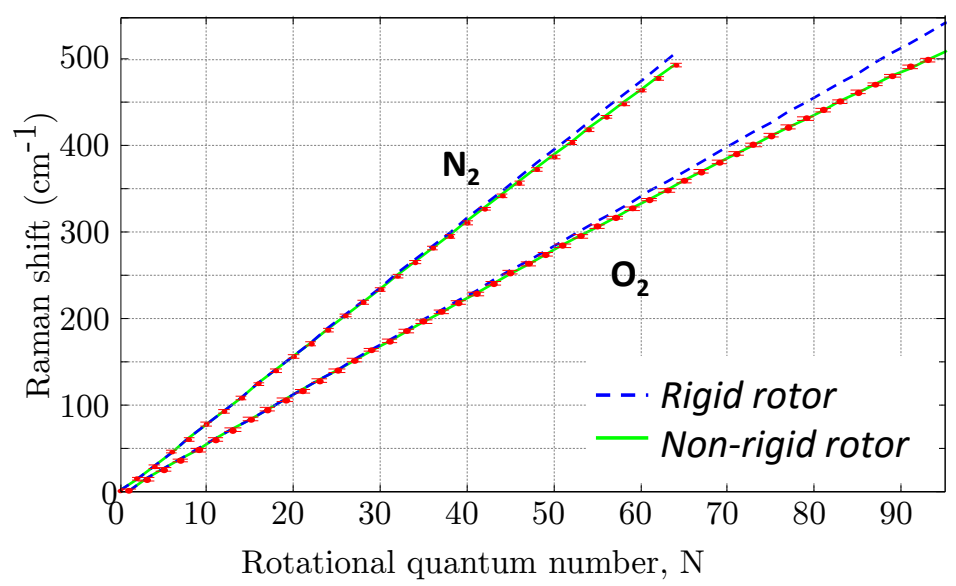

Figure 3.4: Experimental (dots with error bars) and calculated (dashed blue and solid green for the rigid and non-rigid rotor approximations, respectively) rotational energy spectrum expressed as a Raman frequency shift.

because of its nuclear spin statistics.

In a rigid rotor approximation, the rotational energy is $E(N)=B N(N+$ $1)$, where $B$ is the rotational constant of the molecule. This scaling results in a series of equidistant Raman peaks separated by $\Delta \Omega=4 B \Delta N$, with $\Delta N$ being the smallest possible step in the molecular rotational ladder (e.g. 1 for $\mathrm{N}_{2}$ and 2 for $\mathrm{O}_{2}$ ). However, as one can see from the slope of the curves in Fig. 3.4, the measured peak separation does not stay constant, but rather decreases with increasing angular momentum - a direct consequence of the centrifugal distortion evident at $N>50$. Being able to resolve the energies of extreme rotational states, we quantified the magnitude of the centrifugal distortion and verified that it is well described by the Dunham expansion to second power in $N(N+1)$ (green solid lines in Fig. 3.4).

\subsection{Rotational spectroscopy with an optical centrifuge}

As was discussed in subsection 2.3.4, extracting the rotational distributions from REMPI spectra requires the knowledge of both the initial and final states. This means that the electronic spectroscopy of a highly rotationally excited molecule must be understood, both the assignment of resonances and their strengths. This task can be complicated by a predissociative coupling 
and decay behavior in the intermediate excited electronic states [3].

Assuming that the molecule of interest is reasonably stable, REMPI spectra recorded at high temperatures can provide information on the properties of highly excited states, but this approach is limited both by the temperatures that the molecule can tolerate before it dissociates, and by the difficulty of unraveling the complex spectrum of a high temperature molecule, as illustrated by the work done on the spectrum of high temperature water 99. In the case of oxygen, reaching the high rotational states accessed with an optical centrifuge $(N>100)$ would require a temperature of $\approx 50,000 \mathrm{~K}$, while the states accessed in a non-adiabatically laser-kicked ensembles [35] are limited to $\sim 40$ (in the case of oxygen) due to the rapid growth of ionization rate with increasing kick strength. Even if a broad thermal distribution of highly excited rotational states could be produced in a diatomic molecule such as oxygen, the triplet structure of the ground and excited states coupled with two-photon selection rules (for the $C^{3} \Pi_{g}\left(v^{\prime}=2\right) \leftarrow \leftarrow X^{3} \Sigma_{g}^{-}\left(v^{\prime \prime}=0\right.$ ) transition), would result in as many as 21 overlapping rotational branches (see section 2.3), making spectroscopic assignment challenging [122].

Here we utilized the unique capability of the centrifuge to control the level of rotational excitation for the purpose of obtaining and interpreting complex REMPI spectra of oxygen super rotors $(0<N \lesssim 120)$. We excited oxygen to a narrow rotational wave packet whose center is accurately tuned across the broad range of well defined $N$ values. The centrifuge excitation was then followed by a REMPI measurement. Owing to the narrow $N$ distribution, the detected spectrum became significantly less congested, and identifying rotational resonances was greatly simplified. As we have demonstrated in section 3.1, truncating the spectrum of the centrifuge in a Fourier plane of the pulse shaper by a movable shutter (inset to Fig. 2.2 (a)) enabled accurate control of the rotational state of the centrifuged molecules. Characterizing the centrifuge field with the method of cross-correlation frequencyresolved optical gating (XFROG, section 2.1.2) allowed us to calibrate the final rotation speed of the centrifuge, and hence the corresponding molecular angular momentum, as a function of the shutter position. REMPI detection was carried out using narrowband nanosecond probe pulses tunable from $279 \mathrm{~nm}$ to $288 \mathrm{~nm}\left(0.1 \mathrm{~cm}^{-1}\right.$ linewidth, $500 \mu \mathrm{J}, 50 \mathrm{~Hz}$ repetition rate, see subsection 2.3.4 for details).

The two-dimensional REMPI spectrogram of centrifuged oxygen is shown in Fig. 3.5, where the detected ion count is plotted against the probe wavelength (horizontal axis) and the final rotation speed $\Omega$ of the truncated centrifuge (vertical axis). The latter is expressed in terms of the angular momentum $N$ of an oxygen molecule rotating with the angular frequency 


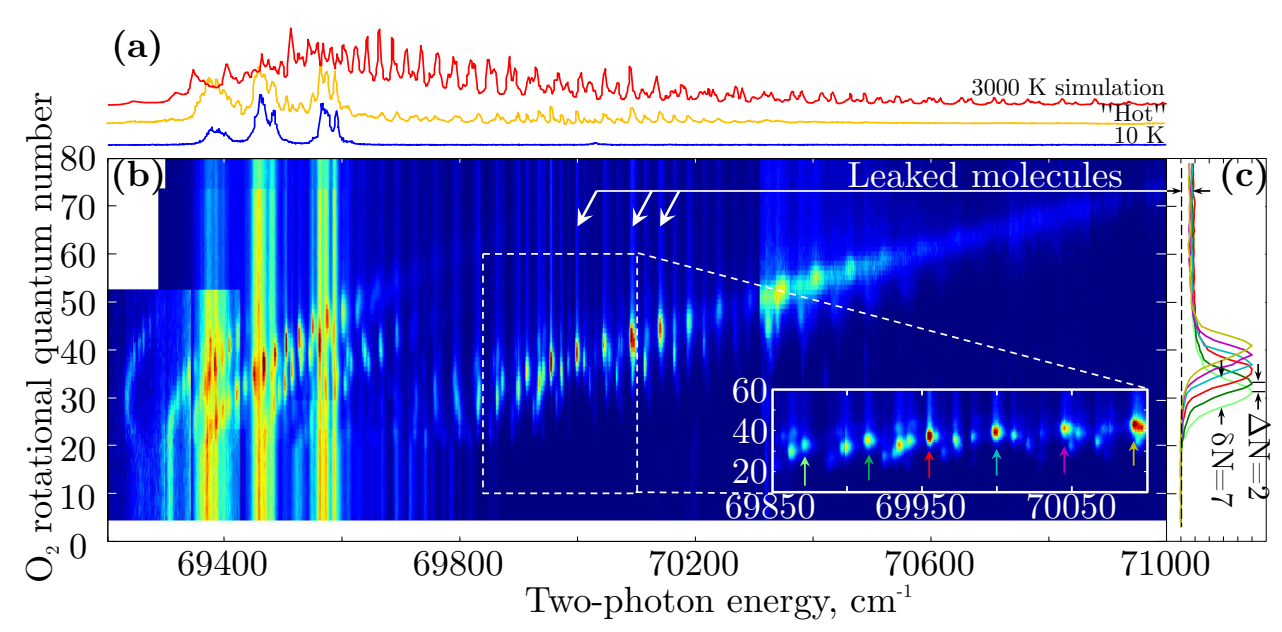

Figure 3.5: 2D REMPI spectrogram for a linearly polarized probe. (a) Experimental spectra of cold $(10 \mathrm{~K}$, blue) and centrifuged molecules (yellow), along with a simulated spectrum of a "hot" thermal ensemble (3000 K, red) calculated with PGOPHER software [134]. (b) Ion signal as a function of the probe laser wavelength and molecular angular momentum defined by the centrifuge final rotation speed. Different areas of the $2 \mathrm{D}$ plot were measured with different sensitivities and probe intensities and are displayed with different color scales to compensate for the broad dynamic range of the data. c Vertical cross-sections of several consecutive peaks from one particular branch, shown in the inset to (b). The peaks are regularly separated with a distance of $\Delta N=2$ reflecting ${ }^{16} \mathrm{O}_{2}$ nuclear spin statistics.

$\Omega$, according to:

$$
\begin{gathered}
\Omega=[\epsilon(N)-\epsilon(N-1)] / \hbar, \\
\epsilon(N)=h c B N(N+1)-h c D N^{2}(N+1)^{2},
\end{gathered}
$$

where $\epsilon$ is the energy of state $|N\rangle$, $c$ is the speed of light in vacuum, $B=$ $1.438 \mathrm{~cm}^{-1}$ and $D=4.839 \times 10^{-6} \mathrm{~cm}^{-1}$ [51].

Each peak in the two-dimensional REMPI spectrogram of Fig. 3.5 (b) corresponds to a two-photon transition between a rotational level in the electronic ground state, $X^{3} \Sigma_{g}^{-}$, and a rotational level of $C^{3} \Pi_{g}$. The finite horizontal width of the observed peaks stems from the predissociation linewidth (as in conventional "1D REMPI" detection), whereas finite vertical spread reflects the narrow width of the excited rotational wave packet created by the centrifuge. 
The complexity of the two-photon absorption line structure in rotationally hot oxygen gas is illustrated by red and yellow lines in Fig. 3.5 (a) which correspond to the hot thermal ensemble (simulated numerically) and the ensemble of centrifuged molecules (experimentally observed 2D spectrogram integrated along its vertical dimension), respectively. In sharp contrast to conventional 1D REMPI spectroscopy, controlled centrifuge spinning offers direct assignment of rotational quantum numbers to the observed REMPI peaks, as well as significantly better peak separation due to their distribution along the added second dimension.

Vertical traces originating from bright resonance peaks in Fig. 3.5 (b) (examples are marked with white arrows) correspond to molecules which "leaked out" of the weakened centrifuge potential before reaching the terminal angular frequency of the centrifuge. After escaping the centrifuge, these molecules continue their free rotation while the trap is accelerating further. The three bright vertical stripes reproduce the initial cold beam spectrum (blue line in Fig. 3.5 (a)) and correspond to the molecules which were not trapped by the centrifuge. The width of the final rotational wave packets can be readily extracted as $\delta N \approx 7$ (FWHM), from the vertical cross sections, shown in Fig. 3.5 (c). The REMPI spectrogram shows an accelerated centrifuge excitation to the rotational states with $N$ as high as $\sim 80$ (dim diagonal trace pointing at the upper right corner in Fig. 3.5 (b)). Rotational line broadening above $N \approx 60$ can be attributed to the increasing Rydberg-valence interaction (governed by the Franck-Condon overlap with the continuum wavefunctions) similarly to the previously observed rotational broadening in the lower vibrational states $\left(v^{\prime}=0,1\right)$ of the excited potential[122].

One can see that the peaks in Fig. 3.5 are grouped in regular patterns, resembling Fortrat parabolas corresponding to different rotational branches. Within a single branch, the center of each consecutive resonant peak is shifted by $\Delta N=2$ (Fig. 3.5 (c)), reflecting the smallest step in the rotational ladder climbing executed by the centrifuge. Circularly polarized light was used to further simplify the spectrum. As shown in Fig. 3.6, the signal strength of different rotational branches depends on the handedness of probe polarization. This is due to the highly non-uniform population distribution among the magnetic sub-levels in the centrifuged wave packet, with most of the population concentrated at $M_{N} \simeq N\left(\right.$ or $\left.M_{N} \simeq-N\right)$ [57].

To identify different rotational branches, we used three sets of molecular constants (for $F_{1}(\Omega=0), F_{2}(\Omega=1)$ and $F_{3}(\Omega=2)$ spin-orbit components of the excited state, respectively, with the quantum number $\Omega$ introduced in section 2.3) from the previous studies on thermally excited 


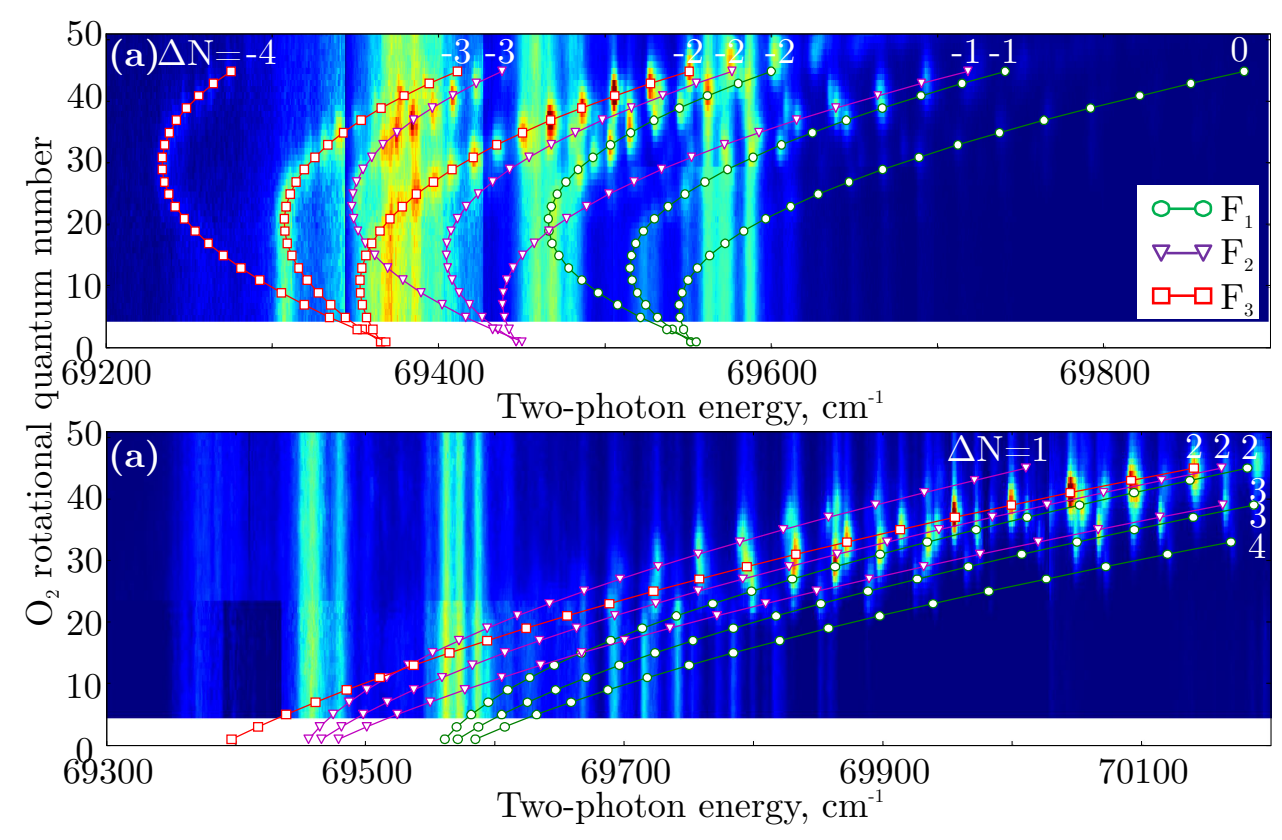

Figure 3.6: 2D REMPI spectrogram for a circularly polarized probe. Electric field vector is counter-rotating (a) and co-rotating (b) with the centrifuged molecules. The directionality of laser-induced rotation results in the sensitivity of the measured signal to the handedness of probe polarization. The results of fitting the data to the theoretical model are shown with colored lines and markers for different branches and resonances, respectively. Branch nomenclature is the same as in [122].

ensembles [82, 135]. These constants are listed in Table 3.1. For $F_{2}$ and $F_{3}$ components, our results are well described by the constants provided by Lewis et al. 82. On the other hand, the observed $F_{1}$ peaks do not agree well with the suggested numerical values $\left(\nu_{0}=69366 \mathrm{~cm}^{-1}\right.$ and $\left.B_{0}=1.6 \mathrm{~cm}^{-1}\right)$, as shown in Fig. 3.7. This can be attributed to the complexity of the broadened and highly overlapping structure of $F_{1}$ lines, which makes it hard to interpret and fit the data from a thermally populated ensemble. Centrifuge spectroscopy enabled us to correct the values of $F_{1}$ molecular constants (Table 3.1 ) by performing the fit of the most pronounced $\Delta N=-2$ branch (Fig. 3.7).

The lowest vibrational level of $C^{3} \Pi_{g}$ electronic state of oxygen which is known to exhibit well-resolved rotational structure is $v^{\prime}=2$ [122. Pre- 


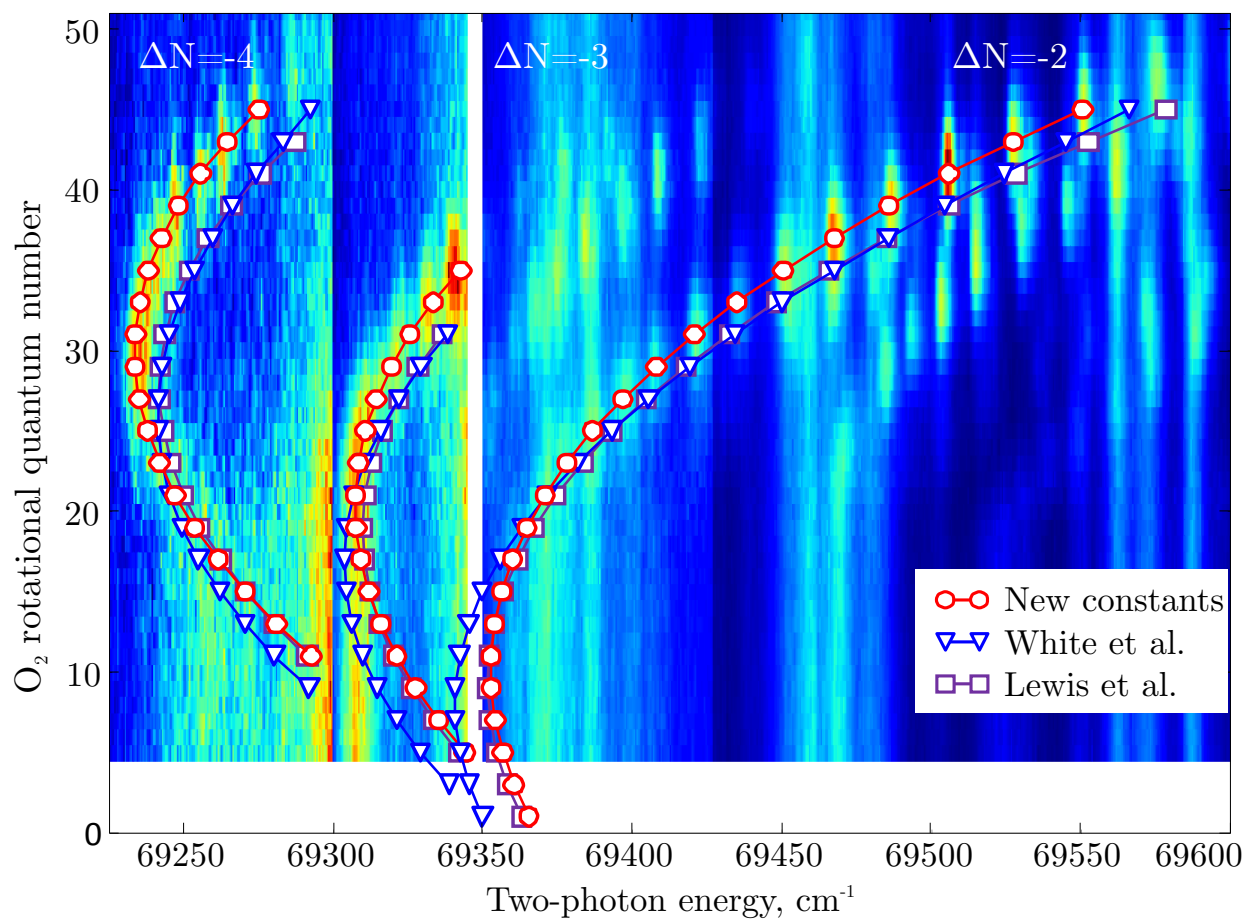

Figure 3.7: Comparison of the observed REMPI data for the perturbed $F_{1}$ spin-orbit component with the calculations based on molecular constants from our work (red circles), White et al. [135] (blue triangles) and Lewis et al.[82] (purple squares)

dissociation to the closely lying valence state $1^{3} \Pi_{g}$ broadens the rotational spectrum of the lower vibrational states $v^{\prime}=0,1$, where the molecule has a high probability of tunnelling to the said continuum state, undergoing dissociation. This broadening is weakened in the case of $v^{\prime}=2$ because the repulsive potential crosses the level near the node of the vibrational wavefunction, lowering the Franck-Condon overlap [122. The FC overlap, however, increases with the increasing degree of rotational excitation. At high values of $N$, we observed a significant line broadening which results in a completely unresolvable rotational structure at $N \gtrsim 60$ (see Fig. 3.5).

At even higher centrifuge frequencies, corresponding to the extreme rotational levels with $99 \leq N \leq 125$, we observed the re-appearance of narrow resonances shown in Fig. 3.8. Their highly non-monotonic line width 
3.3. Mapping out the angular distribution of molecular super rotors

Table 3.1: Molecular constants used to fit the data in Fig. 3.6

\begin{tabular}{llll}
\hline Spin-orbit branch & $\nu_{\mathbf{0}}, \mathbf{c m}^{-\mathbf{1}}$ & $\mathbf{B}_{\mathbf{0}}, \mathbf{c m}^{-\mathbf{1}}$ & $\mathbf{D}, \mathbf{c m}^{-\mathbf{1}}$ \\
\hline${ }^{3} \Pi_{0}\left(F_{1}\right)$ & 69375 & 1.585 & $2.5 \times 10^{-7}$ \\
${ }^{3} \Pi_{1}\left(F_{2}\right)$ & 69445 & 1.648 & $1.0 \times 10^{-5}$ \\
${ }^{3} \Pi_{2}\left(F_{3}\right)$ & 69550 & 1.685 & $1.3 \times 10^{-5}$ \\
\hline
\end{tabular}

dropped down to a well-resolved $\sim 7 \mathrm{~cm}^{-1}$ (Fig. 3.9). Similar linewidth dependencies were previously observed in OD 21$]$ where they were used to analyse the repulsive state. Our analysis showed that, unlike the previously discussed branches of $C^{3} \Pi_{g}\left(v^{\prime}=2\right) \leftarrow \leftarrow X^{3} \Sigma_{g}^{-}\left(v^{\prime \prime}=0\right)$, the observed ultra-high narrow lines originate from the $v^{\prime}=1$ state, which displayed no resolvable rotational structure at lower rotational levels, but re-appeared at higher $J$ 's.

Well described by Hund's case (b) at such high degree of rotational excitation, the observed rotational structure consisted of a series of spin-orbit multiplets. Out of 9 possible $\Delta N$ branches, we observed only two (dashed lines in Fig. 3.8). Given this limited amount of information, fitting the data by a single set of molecular constants proved difficult. Our analysis resulted in two possibilities shown in panels $\mathbf{a}$ and $\mathbf{b}$ of Fig. 3.8 . The retrieved molecular constants are $B_{v}=1.620 \mathrm{~cm}^{-1}$ and $D_{v}=4.4 \times 10^{-6} \mathrm{~cm}^{-1}$ for plot (a), and $B_{v}=1.664 \mathrm{~cm}^{-1}$ and $D_{v}=5.7 \times 10^{-6} \mathrm{~cm}^{-1}$ for plot (b). To choose between the two possibilities, we noted that in Hund's case (a), an effective rotational constant $B_{0}$ for $F_{2}$ spin-orbit component is equal to the true $B_{v}$ value [45]. This implies that at $v^{\prime}=2, B_{v}=1.648 \mathrm{~cm}^{-1}$ (see Table 3.1). Since we expect $B_{v}$ to decrease with $v^{\prime}$, Fig. 3.8 (b) should reflect the correct branch assignment.

\subsection{Mapping out the angular distribution of molecular super rotors}

As discussed in the introduction, the wave functions of a linear rotor's stationary states are given by the spherical harmonics $Y_{J}^{M_{J}}(\theta, \phi)$, with $J, M_{J}$ being the full angular momentum quantum number, and its projection on a laboratory-fixed quantization axis $z$. With a proper choice of $z$, parallel or anti-parallel to the laser propagation direction, the states produced in an optical centrifuge correspond to the wave functions $Y_{J}^{J}(\theta, \phi)=C(J) \sin ^{J} \theta e^{i J \phi}$, where $C(J)$ is a $J$-dependent normalization factor. Angular distributions of these states $P(\theta, \phi)=Y_{J}^{J^{*}}(\theta, \phi) Y_{J}^{J}(\theta, \phi) \sim \sin ^{2 J} \theta$ are shown in Fig. 3.10 
3.3. Mapping out the angular distribution of molecular super rotors

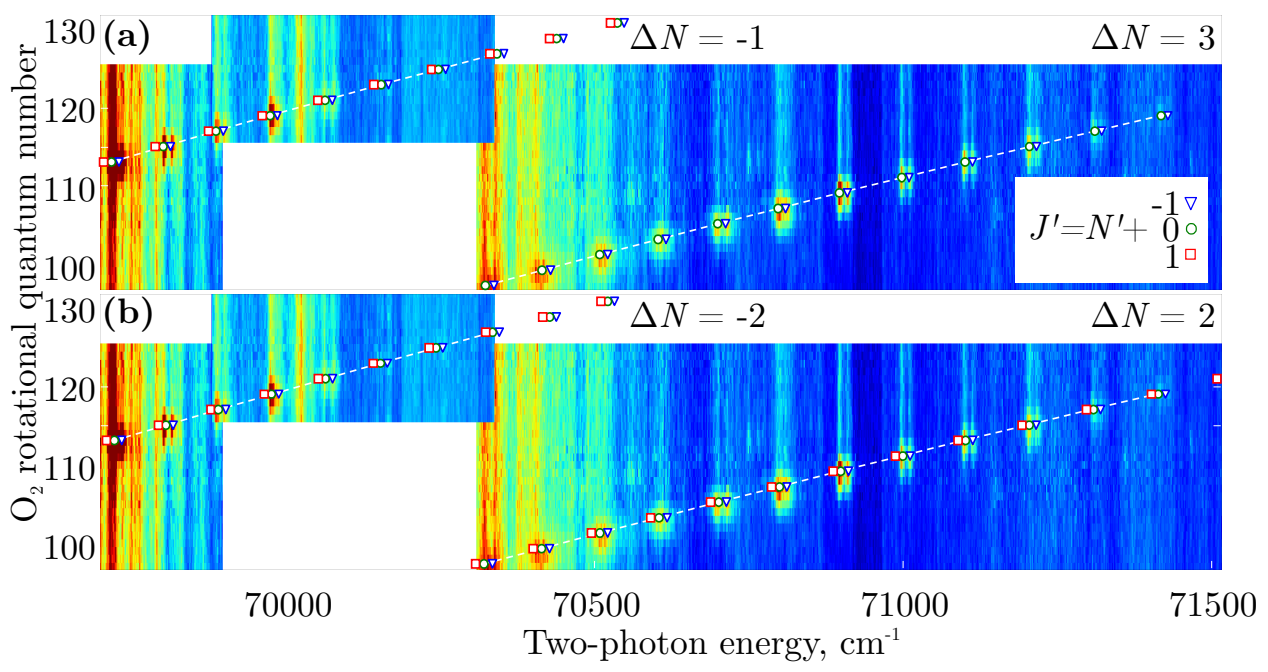

Figure 3.8: Ultra-high rotational resonances of $\mathrm{O}_{2}$. The two panels correspond to two possible ways of fitting the observed resonant branches (apparent along white dashed lines) to the calculated Hund's case (b) structure (labeled with markers). In panel (a), the upper branch corresponds to $\Delta N=-1$, and the lower one to $\Delta N=3$, resulting in $B_{v}=1.620 \mathrm{~cm}^{-1}$ and $D_{v}=4.4 \times 10^{-6} \mathrm{~cm}^{-1}$. In panel (b), the upper branch overlaps with $\Delta N=-2$, whereas the lower one with $\Delta N=2$, yielding $B_{v}=1.664 \mathrm{~cm}^{-1}$ and $D_{v}=5.7 \times 10^{-6} \mathrm{~cm}^{-1}$.

for different values of $J$. As $J$ grows, $\sin ^{2 J} \theta$ becomes more and more concentrated around $\theta=\pi / 2$ in the $x y$ plane, corresponding to the localization of the molecule in the plane of rotation. Since the angular momentum, corresponding to the motion in this plane, is known exactly $\left(M_{J}=J\right)$, the uncertainty principle forbids any knowledge of the angular position $\phi$, leading to the isotropic "pancake"-like in-plane distribution.

Using the velocity map imaging technique (2.4.1), we projected the molecular distribution onto a plane of the detector and saw the effect of an optical centrifuge. Nitrogen gas was cooled down to $10 \mathrm{~K}$ in a supersonic expansion and Coulomb exploded by a short 35 fs laser pulse. The MCP gate was set to the arrival of $\mathrm{N}^{+}$ions. To minimize the effect of the dynamic alignment (discussed in section 2.4), the laser intensity was kept below $10^{15} \mathrm{~W} / \mathrm{cm}^{2}[93$. Choosing the field polarization normal to the plane of detector resulted in centrally symmetric images, as shown in Fig. 3.10 (b), corresponding to the initial isotropic distribution. 


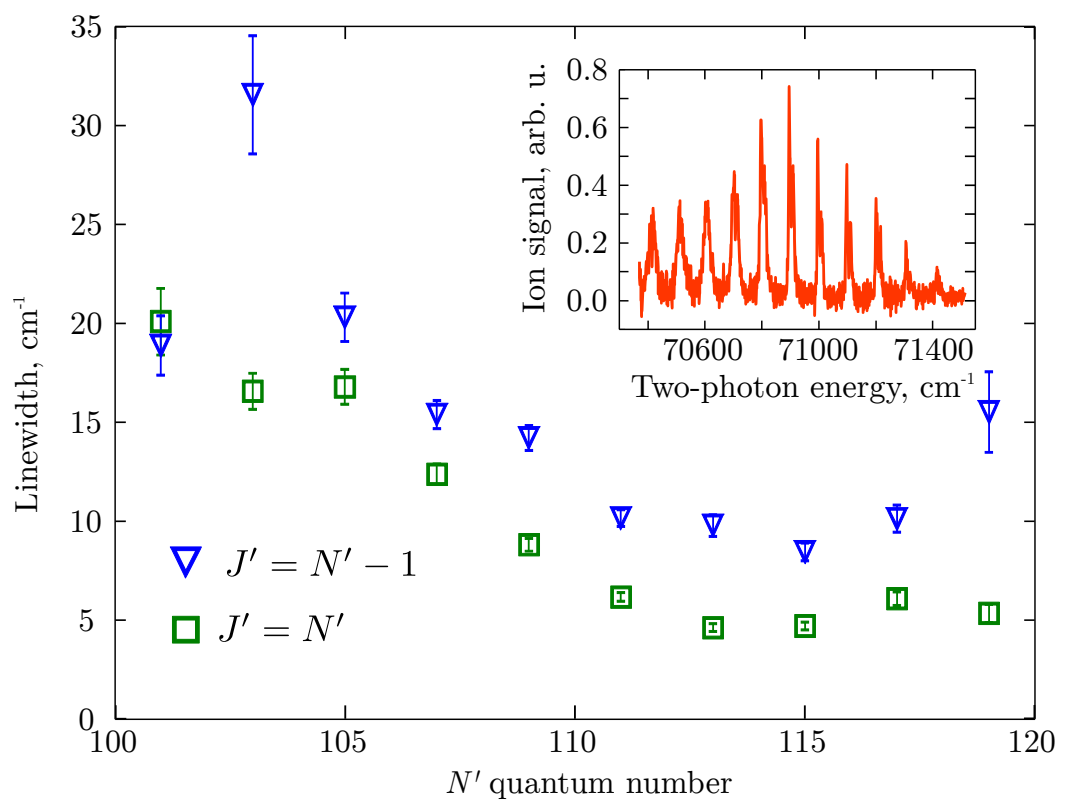

Figure 3.9: Observed linewidths of $J^{\prime}=N^{\prime}-1$ (triangles) and $J^{\prime}=$ $N^{\prime}$ (squares) spin-orbit sublevels of $C^{3} \Pi_{g}\left(v^{\prime}=2\right)$ level as functions of rotational quantum number $N^{\prime}$. Inset demonstrates a fit of experimental data (solid red) to a sum of lorentzians in order to extract linewidths. Absolute position, absolute area and the widths of two peaks were fitted for each doublet individually, with the areas ratio fixed to a value extracted from the best resolved $N^{\prime}=115$ doublet and with a doublet line separation equal to a calculated one.

When the molecules were pre-excited with an optical centrifuge prior to explosion, the images acquired a well-pronounced anisotropic structure, shown Fig. 3.10 (c). The centrifuge was used at the full available bandwidth, bringing the molecules to the rotational level with $J \approx 85$.

An important limitation of using this method for the reconstruction of the exact angular distributions of super rotors stems from the drift effect owing to their high rotational frequencies [70]. The rotational energy of the centrifuged nitrogen reached $1.8 \mathrm{eV} /$ molecule, becoming comparable with the Coulomb explosion energy release of $6.6 \mathrm{eV} /$ molecule 48 . As a result, the recoil velocities of the fragment ions deviated from the internuclear axis of the exploding molecule and the measured velocity distribution no longer represented the true molecular angular distribution. 


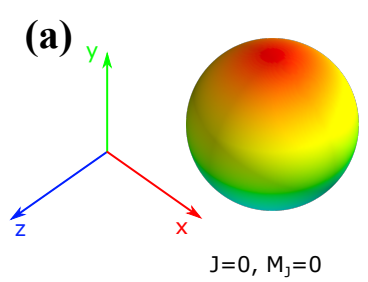

(b)

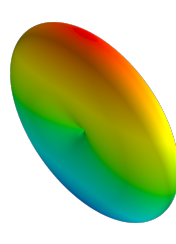

$J=5, M_{J}=5$

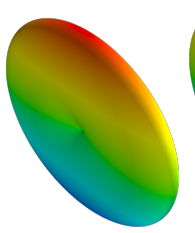

$\mathrm{J}=10, \mathrm{M}_{\mathrm{J}}=10$

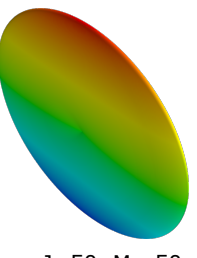

$J=50, M_{J}=50$

(c)
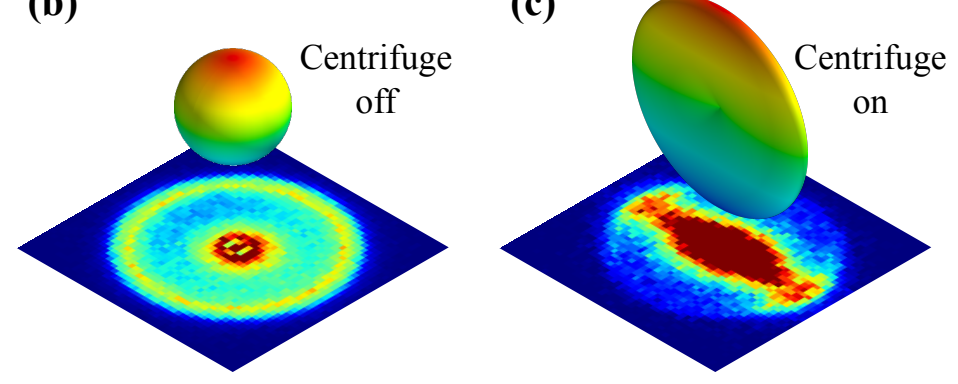

Figure 3.10: (a) Linear rotor angular distributions. Experimental VMI images of $\mathrm{N}_{2}$ fragments with the centrifuge turned off (b) and on (c).

As was discussed in the previous sections on Raman and REMPI spectroscopy, the centrifuge typically populates several consecutive rotational states. The VMI method described here, however, can not be directly used to study their interference. Indeed, let us assume that the centrifuge populates a few rotational states $\Psi(\theta, \phi)=\sum_{J} C_{J} Y_{J}^{J}(\theta, \phi)$. As was explained in subsection 2.4.2, the angle at which the molecules are released are randomly changing from one laser pulse to another. Mathematically, it is equivalent to the application of the operator of finite rotation around $z$ axis by a random angle $\alpha$ to the wavefunction

$$
\Psi_{\alpha}(\theta, \phi)=e^{-i \alpha \frac{\hat{J}_{z}}{\hbar}} \Psi(\theta, \phi)=\sum_{J} C_{J} e^{-i \alpha \frac{J}{\hbar}} Y_{J}^{J}(\theta, \phi)
$$

The corresponding angular distribution

$$
P_{\alpha}(\theta, \phi)=\Psi_{\alpha}(\theta, \phi)=\sum_{J, J^{\prime}} C_{J} C_{J^{\prime}}^{*} e^{-i \alpha \frac{J-J^{\prime}}{\hbar}} Y_{J}^{J}(\theta, \phi) Y_{J^{\prime}}^{J^{\prime *}}(\theta, \phi)
$$

should be averaged over $\alpha$, reflecting an average over many laser shots in the experiments and yielding $P_{\alpha}(\theta, \phi)=\sum_{J}\left|C_{J}\right|^{2}\left|Y_{J}^{J}(\theta, \phi)\right|^{2}$, i.e. an incoherent sum of all $J$-states distributions. To study coherent dynamics of 
3.3. Mapping out the angular distribution of molecular super rotors

the centrifuged-induced distributions, averaging over the randomly changing angle $\alpha$ was eliminated. The results of that study are described in detail in section 4.2 . 


\section{Chapter 4}

\section{Rotational dynamics of molecular super rotors}

A truly adiabatic centrifugal excitation would bring the ground state to one of the excited states $|J\rangle$, corresponding to stationary time-independent distributions. However, as follows from the results shown in sections 3.1 and 3.2 , the real centrifuge excites a wave packet of rotational states. This is attributed to the deviation of centrifugal excitation from the perfect adiabaticity, which leads to broadening of the rotational state wave packet, giving rise to dynamically changing molecular angular distributions. Some of the aspects of this dynamics could be captured in the rotational Raman spectroscopy, by trading off the method's spectral resolution for a finer temporal one (section 4.1). A deeper insight into the field-free evolution of linear molecules was gained using the VMI method. It is described in the context of quantum-classical correspondence in section 4.2.

The initial thermal rotational distribution could further enrich the molecular dynamics by overlapping the motion of several incoherently populated wave packets (section 4.3).

Even for an ideally adiabatic excitation, a stationary $\left|J, M_{J}^{z}=J\right\rangle$ state

could be represented as a superposition of many states $\sum_{M_{J}^{x}=-J}^{J} C_{M_{J}^{x}}\left|J, M_{J}^{x}\right\rangle$ in a different quantization basis (indicated with a superscript in $M_{J}^{x}$ ), degenerate for an isotropic free space. As discussed in section 4.4, this degeneracy could be lifted by an external field, giving rise to yet another source of angular dynamics, and providing an additional way of controlling the molecular rotation.

\subsection{Rotational revivals in the ensembles of molecular super rotors}

The spectroscopic approach used in section 3.1 utilized a narrowband probe, that, on one hand, allowed to resolve individual rotational transitions, but 
on the other hand, lacked the information about their relative phase. To study the rotational motion of super rotors, we used the same setup with a a spectrally wider $(3 \mathrm{~nm}$ instead of $0.1 \mathrm{~nm}$ ) probe pulses, allowing the adjacent Raman lines to interfere with one another, and examined the time dependence of the Raman response. In the time domain, the probe duration was shortened to $500 \mathrm{fs}$, increasing the time resolution and making possible to observe finer wavepacket dynamics.
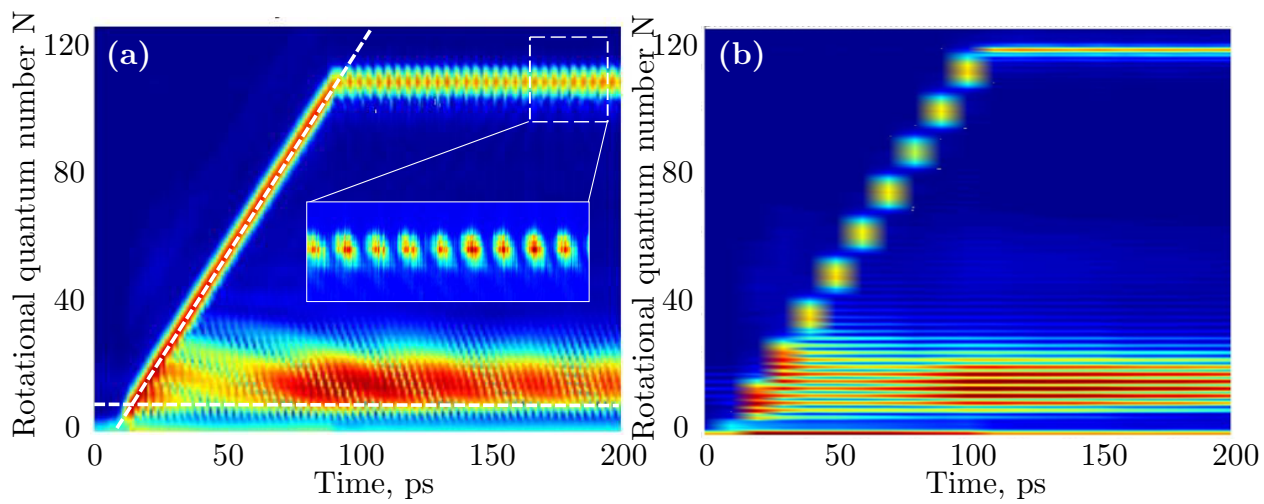

Figure 4.1: (a) Selective centrifuge spinning of oxygen to $N \approx 109$. Dashed tilted line shows the increasing angular frequency of the centrifuge, terminated at about $90 \mathrm{ps}$. Dashed horizontal line marks the most populated rotational state of $\mathrm{O}_{2}$ at room temperature, $N=7$. Oscillations of the coherent rotational wave packet are shown in the inset. (b) Truncating the probe spectrum to $0.1 \mathrm{~nm}$ allowed us to resolve individual rotational Raman transitions, similar to Fig. 3.2, at the expense of losing time resolution.

As shown by a tilted trace in Fig. 4.1 (a), the rotation of molecules trapped in the centrifuge follows the angular frequency of the laser field. A horizontal trace originating at the end of the centrifuge pulse (at around $90 \mathrm{ps}$ ) indicates the free rotation of molecular super rotors, released from the centrifuge. The observed oscillatory signal, emphasized in the inset, is indicative of coherent rotational dynamics. In agreement with the general theory of quantum wave packets[80, 111], the evolution of freely rotating molecules exhibits a clear periodicity. The periods are inversely proportional to the second derivative of $E(N)$ with respect to $N$, i.e. $T=$ $[8 B c(1-6 \epsilon N(N+1))]^{-1}$, where $c$ is the speed of light in vacuum and $\epsilon:=D / B \approx 3 \times 10^{-6}$ is the ratio between the two rotational constants in the Dunham expansion. For a rigid-rotor model $(\epsilon=0)$ of oxygen, this 
would result in the main period of $T=(8 B c)^{-1} \approx 2.9$ ps in agreement with our results for "slow" rotation $(N=7$, along the horizontal dashed line in Fig. 4.1 (a)). Centrifugal distortion of fast super rotors $(N=109)$ results in the stretching of the molecular bond and the correspondingly longer period of $3.8 \mathrm{ps}$, in a nice agreement with a prediction. In contrast, similar measurements done with a $0.1 \mathrm{~nm}$ probing pulse, shown in Fig. 4.1(b), provided resolution of individual rotational transitions, at the cost of time resolution of signal oscillations. An in-depth analysis of the periodic field-free dynamics of linear molecules is provided in the next section.

\subsection{Observation of classical-like molecular rotation}

Microscopic quantum objects behaving in a classical manner are of great interest due to the fundamental aspects of quantum-classical correspondence. The well known examples include coherent states of the quantum harmonic oscillator, slowly spreading Rydberg wave packets [136 and nonspreading Trojan wave packets 86 in highly excited atoms. Classically behaving quantum wave packets preserve their minimum uncertainty shape as they move along the corresponding classical trajectories $[14,56,96]$. In the case of rotating molecules, classical-like behavior implies nondispersing rotation of the molecular wave function with narrow distribution of angular momenta and strong localization around the internuclear axis.

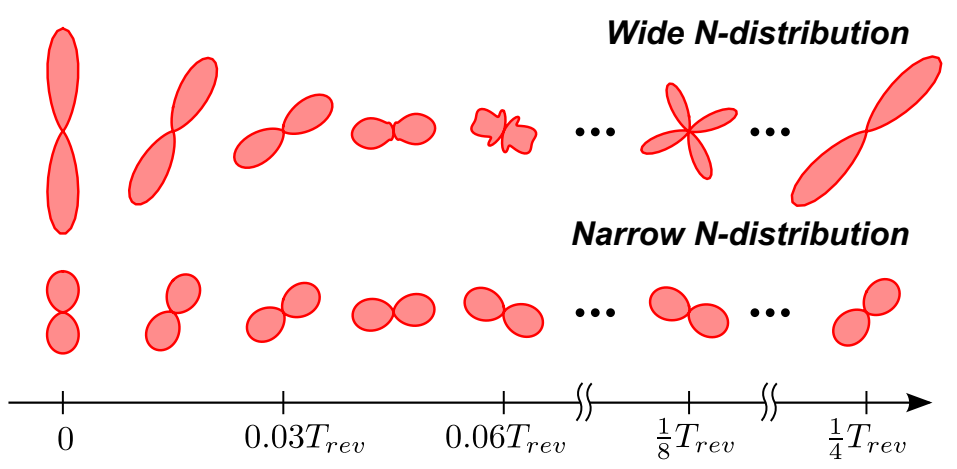

Figure 4.2: Time evolution of a wide (top) and a narrow (bottom) rotational wave packet, consisting of many or only two rotational states, respectively. The latter is an example of a quantum "cogwheel state". $T_{\text {rev }}$ is the rotational revival time. 


\subsection{Observation of classical-like molecular rotation}

Although the popular non-adiabatic approach to molecular alignment with intense non-resonant laser pulses can produce very narrow angular distributions [17, 25, 79, 103, 104, 110, 127, 143], the width of the created rotational wave packet is quite large, making it quickly disperse in angle and undergo oscillations between aligned and non-aligned distributions. Quantum analogs of classical rotational dynamics, such as the gyroscopic destabilization in the rotation of an asymmetric top molecule, has been found in the absorption spectra of thermal molecular ensembles 19. The centrifuge technique enabled us to execute a complete transfer of molecules from the initial ground rotational level to a coherent superposition of a relatively small number of states $\left|N, M_{N}=N\right\rangle$, where $N$ (either only even or only odd) and $M_{N}$ are the rotational and magnetic quantum numbers, respectively. If such wave packet is initially localized along a certain spatial axis (upper left picture in Fig. 4.2), it will rotate with an angular frequency of $\Omega_{N}=\left(\epsilon_{N+2}-\epsilon_{N}\right) /(2 \hbar)$ (where $\epsilon_{N}$ is the rotational energy) while simultaneously undergoing angular dispersion. For a homonuclear molecule with the rotational constant $B$, it will then revive to its initial shape, up to a rotation by a constant angle of $\pi / 4$, at one quarter of the revival time $T_{\text {rev }}=h /(2 B)$ (upper right picture in Fig. 4.2). Indeed, the time-dependent rotational state can be then expressed as

$$
|\Psi\rangle(t)=\sum_{N} C_{N}|N\rangle \exp \left(-\frac{i}{\hbar} \epsilon_{N} t\right),
$$

where $C_{N}$ and $|N\rangle$ are the expansion coefficients and rotational eigenstates, corresponding to an angular momentum $N$. For a homonuclear molecule, $N$ takes either only even or only odd values, depending on the electronic and nuclear spin state of the molecule[47]. For example, only odd $N$ values are allowed in ${ }^{16} \mathrm{O}_{2}$, and the above expression at $t=T_{\mathrm{rev}} / 4$ can be rewritten:

$$
\begin{aligned}
& |\Psi\rangle\left(T_{\text {rev }} / 4\right)=\sum_{n, 2 n-1=N} C_{N}|N\rangle \exp \left(-\frac{i \pi}{4} 2 n(2 n-1)\right) \\
= & \sum_{n, 2 n-1=N} C_{N}|N\rangle e^{-i \pi n(n-1)} e^{-\frac{i \pi}{4}} e^{-\frac{i(2 n-1) \pi}{4}}=e^{-\frac{i \pi}{4}} \sum_{n, 2 n-1=N} C_{N}|N\rangle e^{-\frac{i \pi}{4} N},
\end{aligned}
$$

where in the last expression we used the fact that $n(n-1)$ is always even. The last exponent can be rewritten as $e^{-\frac{i \pi}{4} M_{N}}$, which represents an operator of rotation by $\pi / 4$ along the quantization axis, applied to the initial state $|\Psi\rangle(0)$. The so-called fractional revivals occur at intermediate times $t=$ $\frac{p}{q} \frac{T_{\text {rev }}}{2}$, with $\frac{p}{q}$ being an irreducible fraction $[5,6]$. Provided the number of 


\subsection{Observation of classical-like molecular rotation}

populated rotational states is large, at the time of a fractional revival the initial wave packet splits into $\frac{q}{4}\left(3-(-1)^{q}\right)$ sub wave packets, uniformly distributed along the classical rotational trajectory, which keep rotating with the same frequency $\Omega_{N}$. Indeed, the phases, accumulated by the rotational states at this time are given by:

$$
\begin{array}{r}
\phi(N)=-\frac{B N(N+1)}{\hbar} \frac{p}{q} \frac{h}{4 B}=-\pi n(2 n-1) \frac{p}{q}= \\
-2 \pi n^{2} \frac{p}{q}+\frac{\pi}{2} N \frac{p}{q}+\frac{\pi}{2} \frac{p}{q}, 2 n-1=N .
\end{array}
$$

The two last terms of this sum, as before, correspond to a rotation by a finite angle $\frac{\pi}{2} \frac{p}{q}$ and adding a common phase, respectively. Let us consider the term $\phi_{n}=-2 \pi n^{2} \frac{p}{q}$. It is easy to verify, by inspecting the cases of even and odd values of $q$, that the phase factor $e^{i \phi_{n}}$ is periodic in $n$ :

$$
e^{i \phi_{n+l}}=e^{i \phi_{n}} e^{-2 i \pi \frac{p}{q} l(2 n+l)}=e^{i \phi_{n}},
$$

where the period $l=\frac{q}{4}\left(3-(-1)^{q}\right)$. It can therefore be expanded in the basis of periodic sequences:

$$
e^{i \phi_{n}}=\sum_{k=0}^{l-1} a_{k} e^{-2 i \pi \frac{k}{l} n},
$$

where $a_{k}$ 's are $n$-independent coefficients of this expansion. The wavefunction can therefore be represented, up to an irrelevant phase and a finite rotation by $\frac{\pi}{2} \frac{p}{q}$, as a sum

$$
|\Psi\rangle\left(\frac{p}{q} \frac{T_{\mathrm{rev}}}{2}\right)=\sum_{k=0}^{l-1} a_{k} e^{-2 i \pi \frac{k}{l} \hat{N}}|\Psi\rangle(0) .
$$

For example, around $t=T_{\text {rev }} / 8$, the angular distribution exhibits four peaks in the plane of rotation, as shown in the top row of Fig. 4.2, corresponding to the splitting of the initial wave packet into $l=\frac{4}{4}\left(3-(-1)^{4}\right)=2$ sub wave packets. As a transient effect, such multi-axial alignment has been observed experimentally [25] and used as a main ingredient in the new scheme for quantum logic gates based on the control of rotational wave packets [78, 115].

The nature of the periodic Raman signal oscillation, discussed in the previous section, can now be understood in terms of the rotational Doppler effect (section 2.2). Indeed, around the times 0 and $T_{\text {rev }} / 4$ in Fig. 4.2 (a), the rotating molecular ensemble has highly anisotropic polarizability in the 
plane of rotation, leading to the birefringence in the rotating frame of reference and the appearance of a strong Raman signal. Around $T_{\text {rev }} / 8$ the polarizability becomes isotropic, leading to the disappearance of Raman signal.

If the wave packet consists of only two states $\left|N, M_{N}=N\right\rangle$ and $\mid N+$ $\left.2 n, M_{N}=N+2 n\right\rangle$, where $n$ is an integer, the resulting "cogwheel state" rotates without spreading, preserving its shape at all times [76]. The wave function of a quantum cogwheel is symmetric with respect to rotation by $\pi / n$ and follows the classical-like nondispersing motion indefinitely[140]. The simplest case of a cogwheel state with $n=1$ is shown in the lower row of Fig. 4.2. Similarly to the importance of transient cogwheel distributions in quantum information processing [115, it has been speculated that nondispersing cogwheel states could open new perspectives in the development of molecular machines, whereas in metrology, they could serve as "molecular stopwatches" on a femtosecond time scale with immediate applications in synchronizing ultrafast laser sources [18. The latter could be accomplished by Coulomb exploding two spatially separated ensembles of rotating molecules, and measuring the relative angle of recoil between the ions, produced by different sources. Using an optical centrifuge, we investigated the nondispersing $n=1$ cogwheel state in centrifuged $\mathrm{D}_{2}$ molecules. In oxygen, we created a coherent superposition of more than two rotational states and observed the transition between the classical-like rotation of a molecular stopwatch and that of a quantum cogwheel.

We tracked the rotation of centrifuged molecules by means of the VMI technique described in section 2.4. We combined a beam of circularly polarized 35 fs ionizing probe pulses with a beam of centrifuge pulses. Circular polarization was used to eliminate the effect of the dynamic alignment. Both laser beams were focused on a supersonically expanded gas of either $\mathrm{O}_{2}$ or $\mathrm{D}_{2}$ molecules between the charged plates of the VMI setup. The rotational temperature in the supersonic jet was below $10 \mathrm{~K}$, which in the case of oxygen meant that the majority of molecules were in the ground rotational state, $N=1$. The resulting images, averaged over 10000 laser shots, are shown in Fig. $4.3(\mathbf{a}, \mathbf{b})$. The images consisted of a set of concentric rings, corresponding to different ionization channels. Even in the absence of the centrifugal excitation (panel a), the intensity was higher in the plane of laser polarization (PP). This is due to the geometric alignment effect - the ionization probability is higher for those molecules which happened to lie in the plane of the probe's circular polarization. When the molecules were centrifuged prior to the Coulomb explosion (panel b), the image became even more squeezed towards the polarization plane, because of the molecu- 

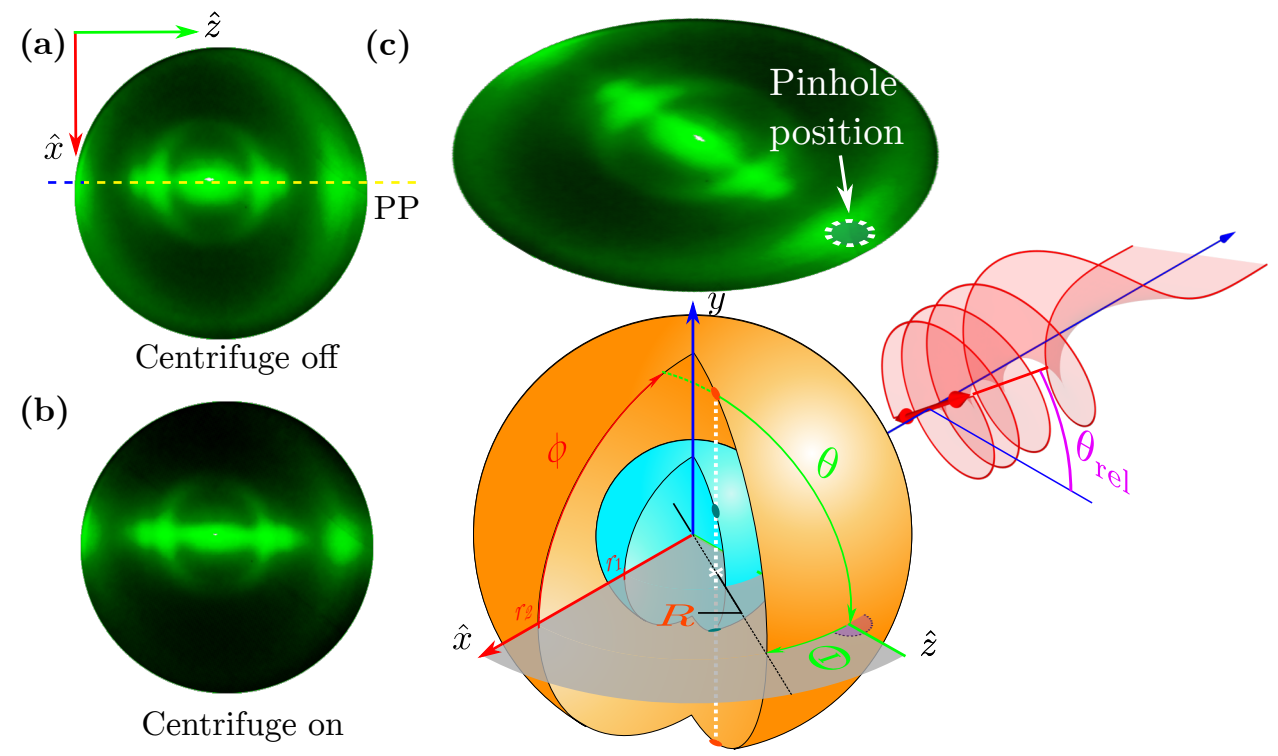

Figure 4.3: Experimental VMI of $\mathrm{O}_{2}$ taken with the centrifuge turned off (a) and on (b). The apparent alignment of the cold ensemble in the plane of probe polarization (PP) in panel (a) is due to the geometric alignment effect. The enhancement of this alignment in panel (b) is caused by the centrifuge. (c) Geometry of the in-plane distribution measurements.

lar localization at that plane.

As was discussed in section 2.4, extracting the exact 3D velocity distribution from a $2 \mathrm{D}$ image is non-trivial, especially in the case of axiallyasymmetric distributions. We developed a new method, that allowed us to estimate the angular distribution in the polarization plane. Assuming, that the kinetic energy release is constant for a given channel, the velocity distribution function from Equation $(2.1)$ can be written as $f(r, \theta, \phi)=$ $\sum_{n} c_{n} \delta\left(r-r_{n}\right) f(\theta, \phi)$, where $c_{n}$ and $r_{n}$ is the fraction of molecules dissociating via the $\mathrm{n}$-th channel and their displacement along the recoil direction by the time of arrival at the detector (see Fig. 4.3 (c) for the definition of the relevant coordinates). Integral (2.1) then simplifies to:

$$
F(R, \Theta)=\sum_{n: r_{n}>R} \frac{c_{n} r_{n}}{\sqrt{r_{n}^{2}-R^{2}}}\left(f\left(\theta_{n}, \phi_{n}\right)+f\left(\theta_{n},-\phi_{n}\right)\right)
$$

where $\theta_{n}=\arccos \left(\frac{R \cos \Theta}{r_{n}}\right)$ and $\phi_{n}=\arccos \frac{\tan \Theta_{n}}{\tan \theta_{n}}$. The physical meaning 


\subsection{Observation of classical-like molecular rotation}

of this formula is illustrated in Fig. 4.3 (c), where the recoil velocities of the two dissociation channels are distributed over the blue and orange spheres. The Abel integral over the projection line (dashed white line) is just a sum over all intersection points with the channel spheres.

We introduced an opaque mask with a pinhole, centered at the circumference of the largest observable dissociation ring in the polarization plane PP, as shown in Fig. 4.3 (c). At this point, $\Theta=0$ and $R=\max \left(r_{n}\right)$, which leaves in (4.4) a single term $\sim f(0, \pi / 2)$, resulting from a single molecular orientation angle at $\theta=0^{\circ}$. The phosphorescence signal, recorded behind this pinhole with a photomultiplier tube (PMT), was proportional to the molecular angular distribution along $\theta=0^{\circ}, f_{\theta_{\text {rel }}}\left(\theta \equiv 0^{\circ}\right)$. The dependence of this distribution on the release angle $\theta_{\text {rel }}$, included in $f_{\theta_{\text {rel }}}(\theta)$ as a parameter, is equivalent to the dependence on $\theta$ at a fixed $\theta_{\text {rel }} \equiv 0$, i.e. $f_{\theta_{\text {rel }}}(0) \equiv f_{0}\left(-\theta_{\text {rel }}\right)$. We investigated the latter as a function of the centrifuge-to-probe delay time. In practice, instead of manually varying $\theta_{\text {rel }}$, we relied on its natural fluctuations due to the centrifuge instability, and measured this angle for every centrifuge pulse, as described in 2.4.2.

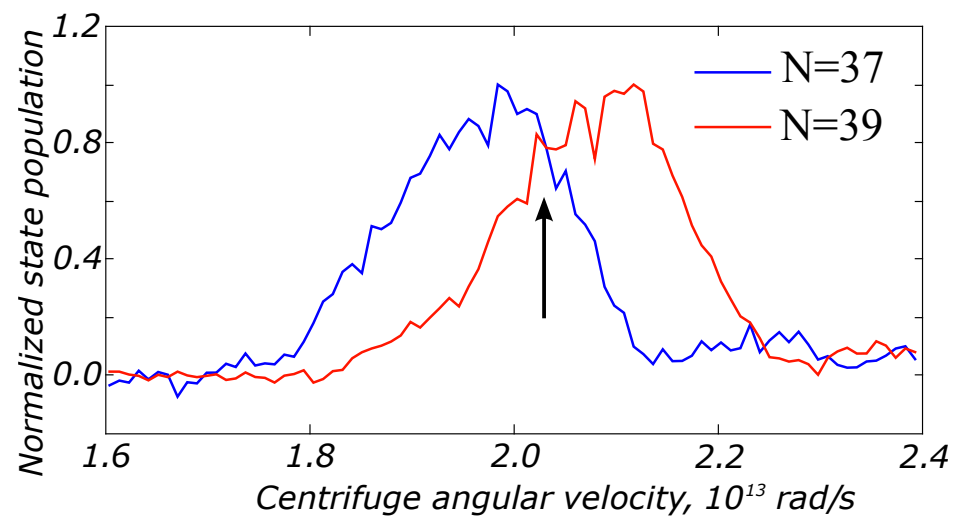

Figure 4.4: Normalized population of the rotational states with $N=37$ (blue) and $N=39$ (red) as a function of the centrifuge final angular velocity. The black arrow shows the terminal angular frequency of the centrifuge used in this work for creating rotational wave packets in $\mathrm{O}_{2}$.

To monitor the free evolution of the rotational wave packets in centrifuged oxygen, we truncated the spectral bandwidth of the centrifuge so as to match its terminal rotational frequency with that of an oxygen molecule occupying rotational states with $N=37$ and $N=39$. The population of 
the two states, measured with REMPI (section 3.2), is shown by the blue and red curves in Fig. 4.4 as a function of the terminal angular frequency of the centrifuge. To make them equally populated, we fixed the latter at the value indicated by the black arrow. Note, however, that since the distance between the two peaks and their half-widths are comparable, the two neighboring states with $N=35$ and $N=41$ were also populated by the centrifuge. The nature of the distribution width could be explained by the non-adiabatic turn-off of the centrifuge field. Indeed, in the frame of reference rotating with the centrifuge, the molecule is in one of the eigenstates of the oscillating electric field potential (the so-called pendular states), with its axis aligned along the field polarization. A sudden turn-off of the field would preserve the instantaneous probability density, leaving the molecule in a superposition of several consecutive $\left|N, M_{N}=N\right\rangle$ states.

Two VMI images of oxygen, one with and one without the centrifuge, are shown in Fig. $4.3(\mathbf{a}, \mathbf{b})$. Pre-excitation with an optical centrifuge resulted in the visible narrowing of the ion distribution in space, owing to the localization of the rotational $\left|N, M_{N}=N\right\rangle$ wave functions in the plane perpendicular to the propagation direction $x$ of the centrifuge (plane 'PP' in Fig. 4.3 (a)). After binning the PMT signal into 11 intervals, uniformly distributed from $\theta_{\text {rel }}=0$ to $\theta_{\text {rel }}=\pi$, we plotted it as a function of the time delay between the centrifuge and the Coulomb-explosion pulses.

The result is shown in Fig. 4.5 (a). Having been aligned at the moment of the release from the centrifuge, the molecules first underwent free rotation with the expected classical frequency of 3.3 full rotations per picosecond, indicated by the blue dashed line at the beginning of the scan and shown with a better resolution in Fig. 4.5 (b). The wave packet, however, gradually dispersed and, although the rotational dynamics could still be identified by the overall linear tilt of the signal, its contrast decreased dramatically. This happened because of the admixture of the neighboring $N=35,41$ states to the rotational wave packet created around $N=37$ and $N=39$.

As expected, the alignment reappeared every quarter-revival time, $\frac{1}{4} T_{\mathrm{rev}}$ (middle section in Fig. 4.5 (a)). In the vicinities of $\frac{1}{8} T_{\text {rev }}$ and $\frac{3}{8} T_{\text {rev }}$, another fractional revival was observed. After repeating the measurement around $\frac{3}{8} T_{\text {rev }}$ with better averaging and higher angular resolution, we plotted the result in Fig. 4.5 (c). Here, instead of two tilted traces per one classical period (marked by a horizontal white bar), reflecting a double-peaked "dumbbell"-shaped alignment geometry, four parallel traces per period can be seen, corresponding to the "cross"-shaped angular distribution peaked in four spatial directions. This observation is well anticipated from the analysis of the rotational dynamics outlined above and illustrated in the top row of 


\subsection{Observation of classical-like molecular rotation}

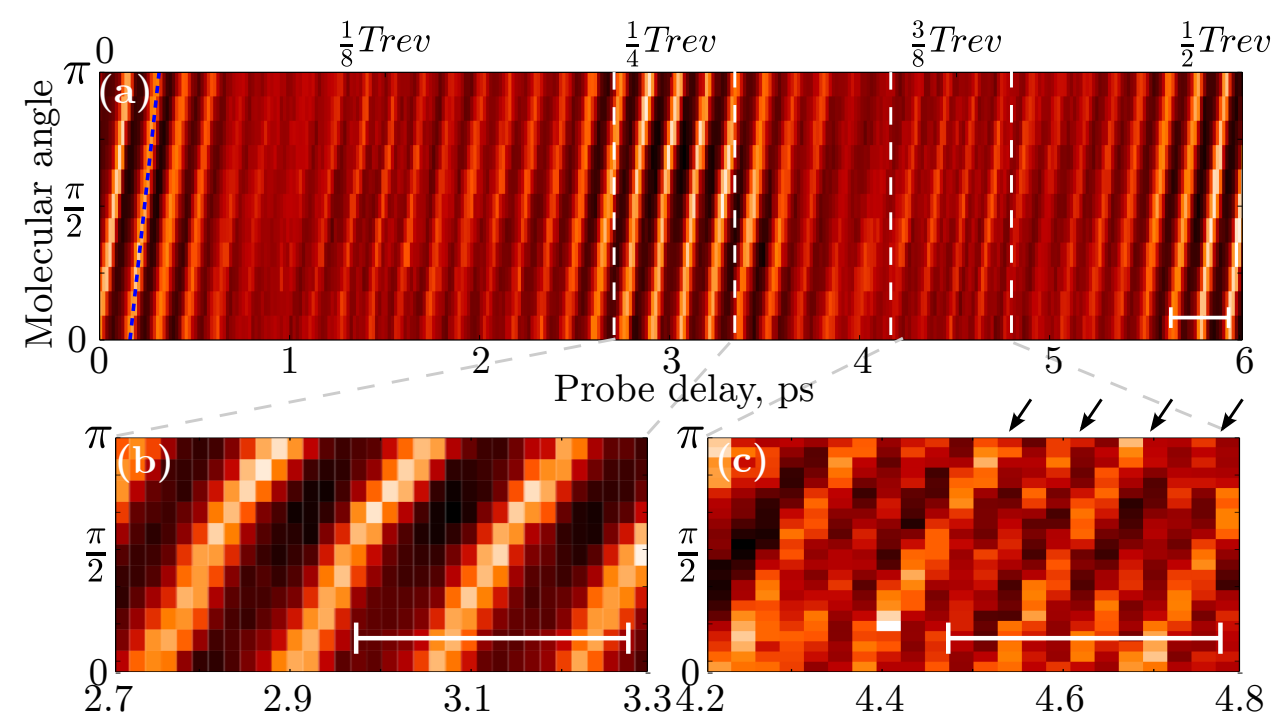

Figure 4.5: (a) Probability density as a function of the molecular angle and the free propagation time. Time zero corresponds to approximately 100 ps since the release from the centrifuge. The blue dashed line marks the calculated trajectory of a "dumbbell" distribution rotating with the terminal angular frequency of the centrifuge, whose classical period is indicated with the white horizontal bar at the lower right corner. Zoom-in to the region near (b) $\frac{3}{8} T_{\text {rev }}$ and (c) $\frac{3}{8} T_{\text {rev }}$, taken with better averaging and angular resolution. Twice higher density of the tilted lines in panel (c) (4 per classical period indicated by the tilted arrows) stems from the emergence of a "cross"-shaped distribution with four lobes along two perpendicular directions.

Fig. 4.2 .

To create a truly nondispersing quantum cogwheel state, one needs to excite a narrower wave packet, truly consisting of only two $N$-states. To achieve this, one can use a gas of lighter molecules with a larger energy spacing between the rotational levels. We repeated the experiment with molecular deuterium, whose moment of inertia is $\sim 20$ times lower than that of oxygen. $\mathrm{D}_{2}$ has two spin isomers, ortho- and paradeuterium, with only even or only odd rotational quantum numbers in their rotation spectrum, respectively. The terminal frequency of the centrifuge was set at half the frequency of the $N=2 \rightarrow N=4$ transition. Under these conditions, orthodeuterium was excited to the coherent superposition of equally populated $N=2$ and $N=4$ states, while paradeuterium was transferred into a 


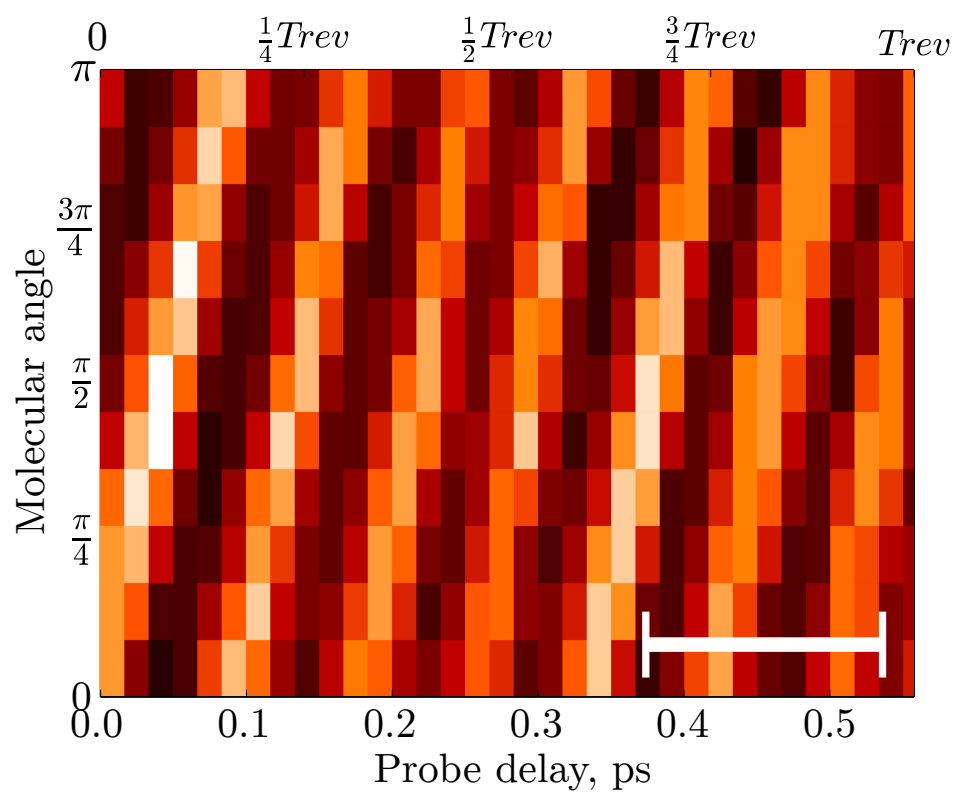

Figure 4.6: Probability density as a function of the molecular angle and the the free propagation time of $\mathrm{D}_{2}$ prepared in the equal-weight superposition of $N=2$ and $N=4$ states. The observed nondispersing behavior illustrates the main property of a quantum cogwheel state. The classical period is indicated with the white horizontal bar at the lower right corner.

single state with $N=3$. As the probability density of the latter does not dependent on the polar angle $\theta$, it introduces a homogeneous background which did not affect the observed dynamics of orthodeuterium, shown in Fig. 4.6. One can see that during one revival time, the molecule completes exactly $3 \frac{1}{2}$ full rotations in agreement with the expected frequency of classical rotation $\Omega_{2}=\frac{1}{2 \pi} \frac{\omega_{4}-\omega_{2}}{2}=7 \frac{B}{h}=\frac{7}{2} \frac{1}{T_{\text {rev }}}$. We observed no dispersion in the angular shape of the created wave packet, as anticipated for the time evolution of a cogwheel state.

\subsection{Coherent spin-rotational dynamics in oxygen}

Among simple diatomic molecules, $\mathrm{O}_{2}$ stands out because of its nonzero electron spin $(S=1)$ in the ground electronic state, $X^{3} \Sigma_{g}^{-}$. The interaction between the spins of the two unpaired electrons and the magnetic field of the rotating nuclei results in the spin-rotation (SR) coupling on the order of 
a few wave numbers, which grows with increasing nuclear rotation quantum number $N[45]$. This coupling of the electron magnetism with molecular rotation, readily controllable with laser light, offers new opportunities for controlling molecular dynamics in external magnetic fields [40].
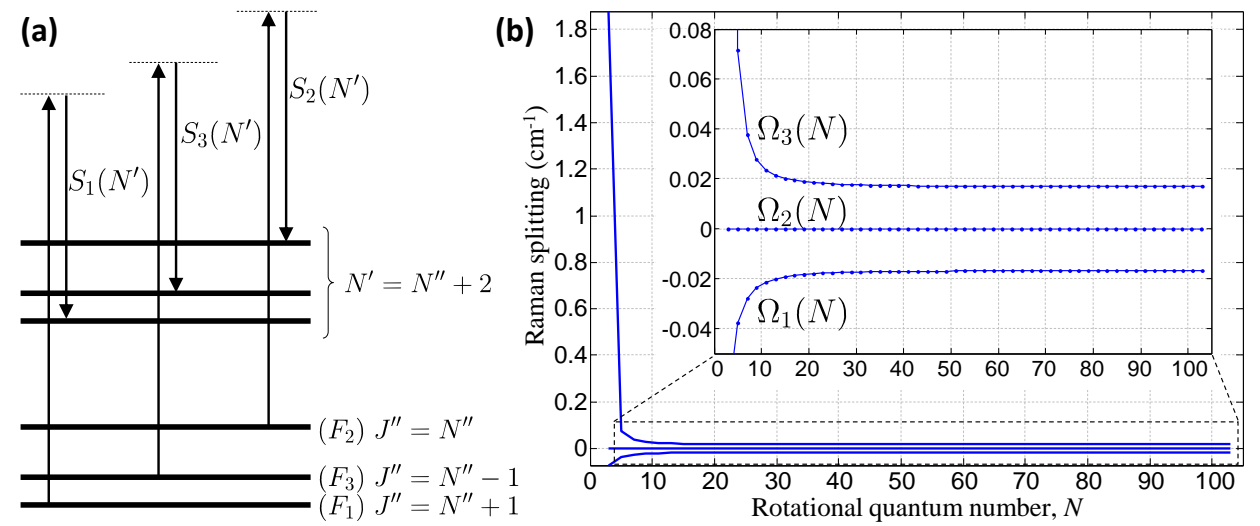

Figure 4.7: (a): Spin-rotational splitting of two rotational levels of oxygen, $N^{\prime \prime}$ and $N^{\prime}=N^{\prime \prime}+2$. Each level is split into three sub-levels with energies $F_{k}, k=1,2,3$ for the total angular momentum $J=N+1, N, N-1$, respectively. Three strongest Raman transitions (out of the total six allowed by the selection rules) corresponding to the $S\left(N^{\prime}\right)$ branch are shown and labeled according to the participating $J$-states. (b): Dependence of the three Raman frequencies ( $\Omega_{k}$ for $S_{k}$ line) on the rotational quantum number.

SR coupling splits each rotational level in three (Fig. 4.7 (a)), with the total angular momentum $J=N, N \pm 1$ [45]. The energy splitting, originally observed by Dieke and Babcock in 1927[24] and later calculated by Kramers [72 and Schlapp[107], is currently known with a very high degree of accuracy[137]. Though routinely observed in the frequency domain with the methods of microwave spectroscopy $[8,91]$, the spin-rotation splitting and the associated with it SR dynamics have not been previously studied in the time domain.

We employed the method of time-resolved coherent Raman spectroscopy (section 3.1) to study this dynamics. Because of the spin-rotation coupling, any $N \rightarrow(N+2)$ Raman transition in oxygen consists of six separate lines belonging to one $Q$, two $R$ and three $S$ branches with $\Delta J=0,1$ and 2, respectively. The strength of both $Q$ and $R$ branches drops quickly with increasing $N$ and becomes negligibly small at $N>5[7]$. The frequency 
difference between the lowest $R$ lines, $R(1)$ and $R(3)$, is about $2 \mathrm{~cm}^{-1}$. Their interference in the time domain results in the oscillations with a period of $\approx 17 \mathrm{ps}$, which has been observed experimentally $[90$. On the other hand, the three stronger $S$ branches shown in Fig. 4.7(a) are split by less than 0.05 $\mathrm{cm}^{-1}$ (Fig. 4.7 (b)), which corresponds to the oscillation period of about 600 ps. This time scale is much longer than the collisional decoherence time of the thermally populated rotational levels at ambient pressure [7, 92], explaining why no spin-rotational dynamics has been seen for $N>3$ in the time-resolved experiments 90 .

We employed the technique of an optical centrifuge to excite oxygen molecules to ultra-high angular momentum states, reaching rotational quantum numbers as high as $N=109$. Due to the substantially increased rotational decoherence time at high $N$ (see section 6.1), the detection of spinrotational oscillations was possible even at the pressure of 1 atmosphere. By lowering the pressure, we observed SR dynamics in the broad range of angular momentum, $3 \leqslant N \leqslant 109$.

For this study, we used the experimental setup developed for the coherent Raman spectroscopy of super rotors (section 3.1). Probe pulses were narrowed down to $3.75 \mathrm{~cm}^{-1}$ (FWHM) to allow the resolution of individual rotational lines.

As discussed earlier, the centrifuge-induced coherence between the states $\left|J, M_{J}=J\right\rangle$ and $\left|J+2, M_{J}=J+2\right\rangle$ results in the Raman frequency shift of the probe field. The Raman spectrum of the probe pulses scattered off the centrifuged molecules was measured with a spectrometer as a function of the probe delay relative to the centrifuge. An example of the experimentally detected Raman spectrogram is shown in Fig. 4.8 (a). While spinning up, the molecules were "leaking" from the centrifuge. Narrow probe bandwidth enabled us to resolve individual rotational states and make an easy assignment of the rotational quantum numbers to the observed spectral lines as shown in Fig. 4.8 (b) for the Raman spectrum taken at $t=200 \mathrm{ps.} \mathrm{The}$ created wave packet consisted of a large number of odd $N$-states, with even $N$ 's missing due to the oxygen nuclear spin statistics. Each Raman line underwent quasi-periodic oscillations due to the interference between the three frequency-unresolved components $S_{1,2,3}(N)$ of the $S(N)$ branch split by the spin-rotation interaction. An example of these spin-rotation oscillations for the $N=91$ Raman line is shown in Fig. 4.8 (c). The oscillations start at around $100 \mathrm{ps}$, after the super rotors with the rotational angular momentum of $91 \hbar$ have escaped from the centrifuge.

The intensity of a Raman line corresponding to the transition between 

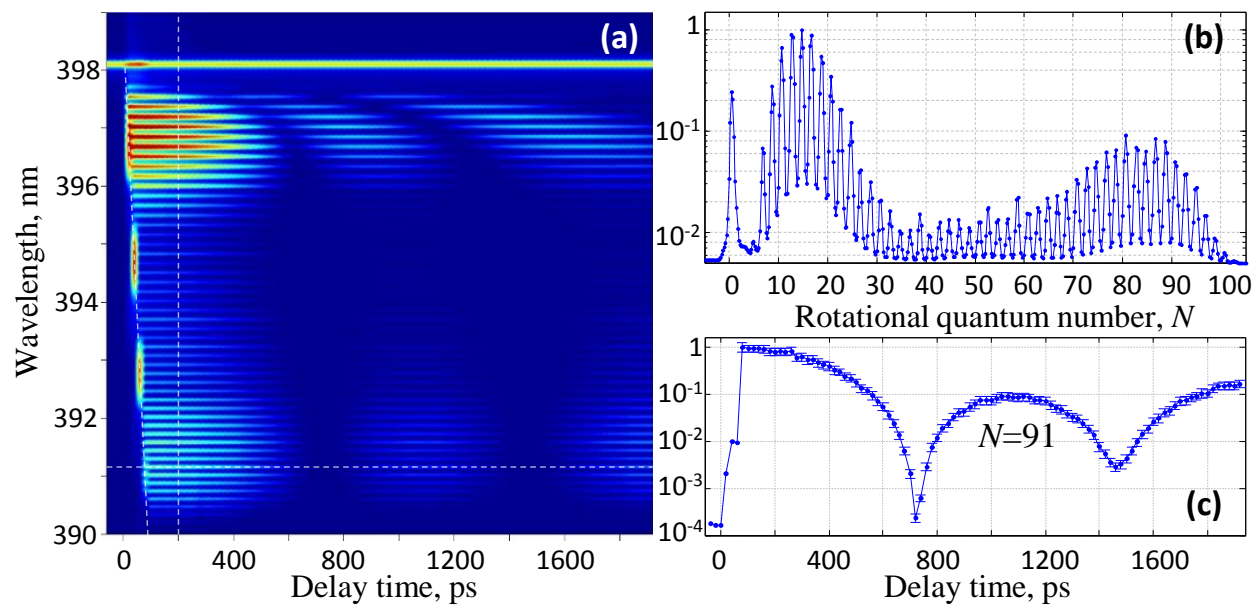

Figure 4.8: (a): Experimentally detected Raman spectrogram of centrifuged oxygen showing the rotational Raman spectrum as a function of the time delay between the beginning of the centrifuge pulse and the arrival of the probe pulse. Color coding is used to reflect the signal strength in logarithmic scale. (b): Cross-section of the two-dimensional spectrogram at the delay of 200 ps (vertical dashed line in a), showing an ultra-broad rotational wave packet created by the optical centrifuge. (c): Spin-rotation oscillations of the $N=91$ Raman line (horizontal dashed line in a). Experimental uncertainty is indicated by the vertical error bars. Note logarithmic scale in all panels.

the states $N$ and $N-2$ can be described as

$$
I_{N}(t)=I_{0}\left|\rho_{N, N-2}(t)\right|^{2} e^{-t / \tau_{N}},
$$

where $I_{0}$ is determined by a number of time-independent parameters, such as molecular concentration and probe intensity, $\tau_{N}$ is the collisional decay time constant and $\rho_{N, N-2}(t)$ is the centrifuge induced coherence between the corresponding rotational states. As discussed above, at $N \geqslant 5$, the latter consists of three main frequency components corresponding to the three $S$ branch transitions (see Fig. 4.7),

$$
\rho_{N, N-2}(t)=\sum_{k=1,2,3} a_{k} e^{i \Omega_{k}(N)\left(t-t_{0}\right)},
$$

with amplitudes $a_{k}$ and frequencies $\Omega_{k}(N)$. Time $t_{0}\left(0<t_{0}<100 \mathrm{ps}\right)$ represents the release time of the corresponding rotational state from the 
centrifuge. For any $N$, the three frequencies are simply

$$
\Omega_{k}(N)=\left[F_{k}(N)-F_{k}(N-2)\right] / \hbar,
$$

where $F_{k}(N)$ are the well known spin-rotational energies of oxygen[45]. After normalizing each measured Raman line to 1 at $t=100$ ps (i.e. shortly after the end of the centrifuge pulse), we fitted the theoretical expression to the observed signals using the following five fitting parameters $\left\{a_{1}, a_{2}, a_{3}, t_{0}, \tau_{N}\right\}$. As demonstrated by a few examples in Fig. 4.9, the oscillatory behavior of our experimental data is well described by Eq.4.5 over the whole range of angular momentum accessed by the centrifuge, from $N=5$ to $N=109$. Note that the weaker the line (e.g. $N=5$ ) the smaller the dynamic range, ultimately determined by the sensitivity of our detector.

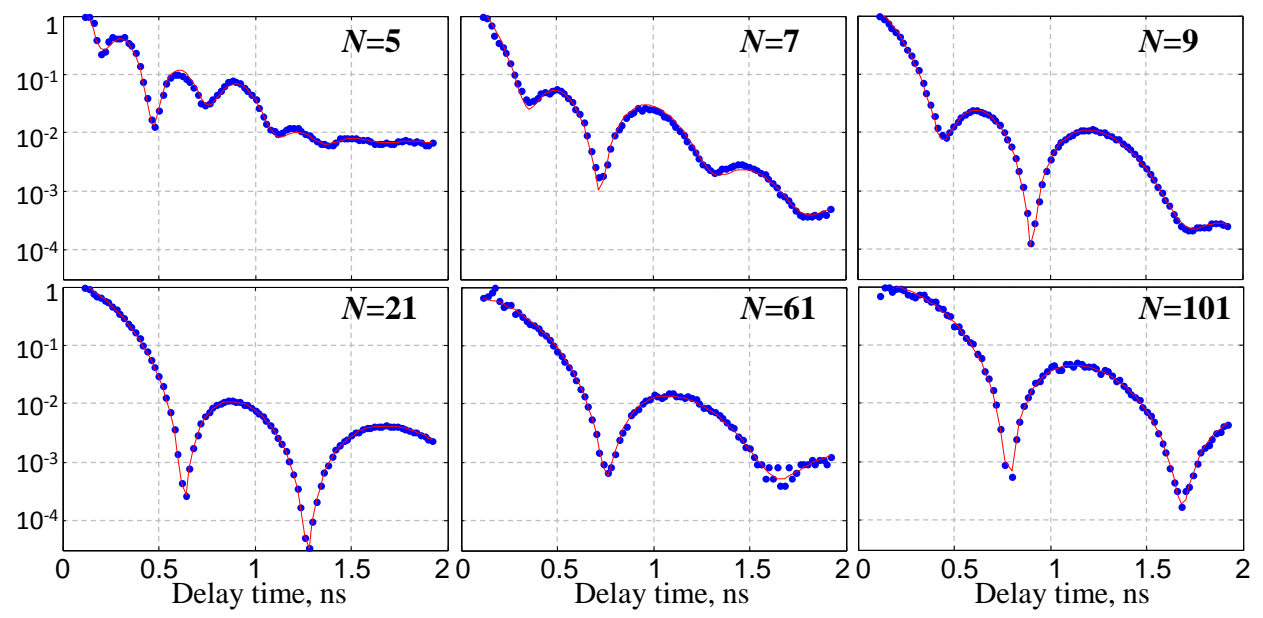

Figure 4.9: The observed data (blue circles, normalized to 1 at $t=100$ ps) and the fit to spin-rotation oscillations (red curves, Eq.4.5) for six different Raman lines corresponding to the rotational quantum numbers $N=5,7,9,21,61$ and 101. Experimental uncertainty (not shown) is similar to Fig. 4.8 (c). Note logarithmic scale in all panels.

\subsection{Dynamics of super rotors in external magnetic fields}

Magnetic field is one of the most powerful tools for controlling atomic and molecular dynamics. Both translational and rotational degrees of freedom of 
molecules have been successfully manipulated via various types of magnetic interaction. Alignment of molecular axes has been accomplished by creating pendular states in paramagnetic molecules at low temperature [37, 117]. Gyroscopic precession along the direction of the applied magnetic field has been predicted for non-magnetic molecules in dispersionless "cogwheel" states [76, 140].

As discussed above, the technique of an optical centrifuge proved capable of producing synchronously rotating molecules with narrow rotational state distribution in a wide variety of species up to extremely high angular frequencies. However, the spatial orientation of the induced angular momentum is defined by the propagation direction of the excitation laser beam, and is therefore not easy to manipulate. To gain control over the axis of the induced molecular rotation, we applied an external magnetic field to molecular super rotors and exploited the coupling between the rotation of a molecule and its magnetic moment. An applied magnetic field causes this moment to precess, "dragging" the axis of rotation with it. The method is applicable to the molecules with magnetic moments of different nature, e.g. due to the rotation of the molecular frame or due to the electronic spin.

Consider a Hund's case (b) molecule possessing an electronic spin, such as molecular oxygen ${ }^{16} \mathrm{O}_{2}$ in the ground electronic state. Its electronic spin is strongly coupled to the molecular angular momentum $\mathbf{N}$. In an external magnetic field, sufficiently weak for not causing the spin to decouple from $\mathbf{N}$, the angular momentum will precess with frequency [31]

$$
\Omega=\mu_{B} g S_{N}|\mathbf{B}| \frac{1}{\hbar N},
$$

where $\mu_{B}$ is the Bohr's magneton, $g$ is the electron g-factor, $\mathbf{B}$ is the magnetic field strength, $S_{N}$ is the projection of an electronic spin on $\mathbf{N}$ and $\hbar$ is the reduced Plank's constant. The $1 / N$ dependence in the above expression comes from the gyroscopic effect: at higher angular momenta, it becomes harder and harder for the same torque (determined by the product of the magnetic moment $\mu_{B} g S_{N}$ and the field strength $\mathbf{B}$ ) to change the rotational axis.

If the molecule does not have an electronic spin, its magnetic moment may stem from the electronic and nuclear currents (equal in magnitude and opposite in direction). The magnitude of both currents can be written as $Z e \nu$, where $Z$ is the electron (proton) number of the molecule, $e$ is the elementary charge and $\nu$ is the rotational frequency. The latter can be expressed through the molecular angular momentum $\mathbf{N}$ as $\nu=\frac{\hbar N}{2 \pi I}$, where $I$ is the molecular moment of inertia. The circular currents from the electrons 
and nuclei will produce magnetic moments $\mu_{\text {elec }}$ and $\mu_{\text {nucl }}$ in the opposite directions, adding to:

$$
\mu_{\text {total }}=\mu_{\text {elec }}+\mu_{\text {nucl }}=-Z e \nu A_{\text {elec }}^{\text {eff }}+Z e \nu A_{\text {nucl }}^{\text {eff }}=\frac{Z e \hbar N}{2 \pi I}\left(A_{\text {nucl }}^{\text {eff }}-A_{\text {elec }}^{\text {eff }}\right),
$$

where $A_{\text {nucl }}^{\text {eff }}$ and $A_{\text {elec }}^{\text {eff }}$ are the effective areas, encircled by the nuclei and electrons, respectively. Notice, that $A_{\text {nucl }}^{\text {eff }} \sim A_{\text {elec }}^{\text {eff }} \sim \pi r^{2}$, and $I \sim n m_{p} r^{2} / 2$, where $r$ is the internuclear distance of the molecule, $n$ is its mass in the atomic units, $n \sim 2 Z$, and $m_{p}$ is the mass of a proton. The magnitudes of both contributing magnetic moments can be estimated as:

$$
\left|\mu_{\text {elec,nucl }}\right| \sim \frac{Z e \hbar N}{4 \pi Z m_{p} r^{2} / 2} \pi r^{2}=\mu_{N} N,
$$

where $\mu_{N}$ is the nuclear magneton. This suggests, that the total magnetic moment would be of the same order, $\mu_{\text {total }}=\mu_{N} g_{r} N$, where $g_{r}$ is the socalled rotational g-factor [32, 52], specific for a given molecule. For typical molecular systems it takes both positive and negative value of order of one. For example, for a ground state of $\mathrm{N}_{2}$ it is -0.2681 [20]. In the presence of an external magnetic field, this magnetic moment will lead to an effective interaction Hamiltonian $H_{m}=\mu_{N} g_{r} \mathbf{N} \cdot \mathbf{B}$ [32, 52, which gives rise to the precession with frequency [140]

$$
\Omega=\mu_{N} g_{r}|\mathbf{B}| \frac{1}{\hbar} .
$$

As the magnetic moment, and hence the torque, grows together with the angular momentum, no $N$-dependence is expected in this case. The precession frequency, however, is a few orders of magnitude lower compared to the magnetic molecules, due to the large difference between $\mu_{N}$ and $\mu_{B}$.

Using the technique of an optical centrifuge, we excited $\mathrm{O}_{2}$ and $\mathrm{N}_{2}$ molecules to high rotational states and observed the dynamics of their angular wave function in the magnetic field applied perpendicular to the rotation axis. By imaging the plane of molecular rotation with ion imaging, we followed its evolution in time and observed two different mechanisms of magnetic interaction, described above.

The imaging was carried out using the same VMI setup described in section 2.4, with a modification to include magnetic field. A circular hole was cut in the bottom electrode of the TOF spectrometer (Fig. 4.10 (a)), through which a cylindrical rare-earth permanent magnet was introduced. The magnetic field at the center was roughly measured with a gaussmeter to 


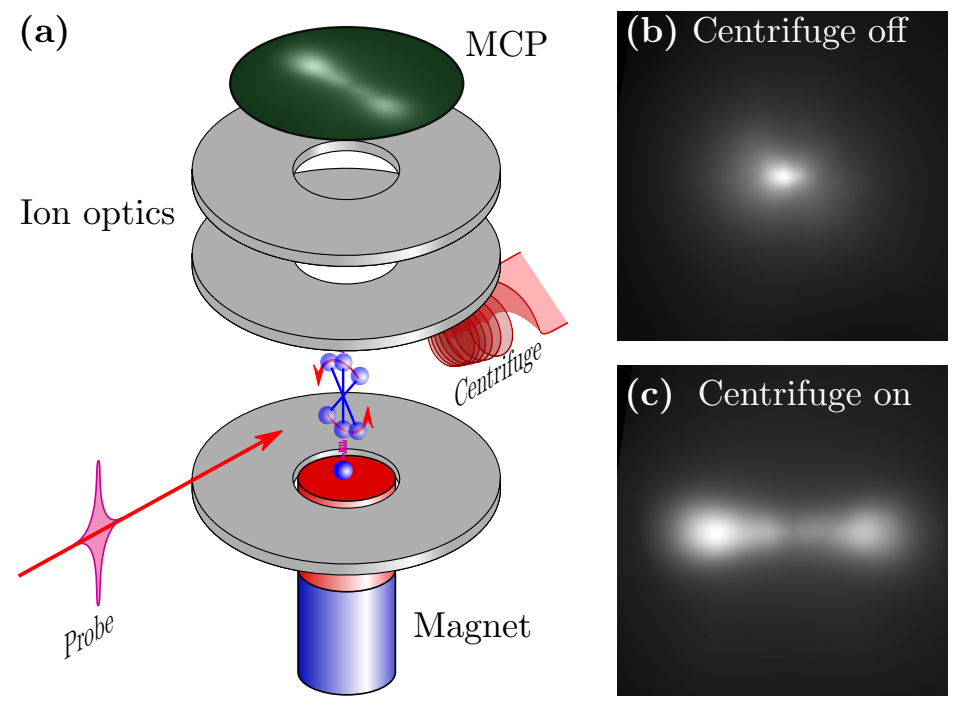

Figure 4.10: (a) Experimental setup for detecting the plane of molecular rotation. The molecules are excited with a centrifuge pulse, then rotate freely in a magnetic field of a permanent magnet, and finally are ionized with a femtosecond probe pulse. The resulting atomic ions are extracted and focused with ion optics of a velocity map imaging (VMI) apparatus onto a microchannel plate detector (MCP) with a phosphor screen. The images observed without (b) and with (c) the rotational excitation by a centrifuge pulse.

be around $350 \mathrm{mT}$ at the molecule-centrifuge interaction region. Nitrogen or oxygen gas was supersonically expanded through a pulsed nozzle, which cooled most of the molecules down to their ground rotational state. The molecules were then excited with a centrifuge pulse to high (up to $10 \mathrm{THz}$ ) angular frequencies.

After evolving in the applied magnetic field for a certain time period $t$, the molecules were exposed to an intense 35 -femtosecond probe pulse, which lead to Coulomb explosion, discussed in detail earlier. Gating the voltage on the MCP allowed us to mass-select only doubly-ionized atomic ions, either $\mathrm{O}^{2+}$ or $\mathrm{N}^{2+}$.

For a given probe pulse energy $\left(\sim 10^{15} \mathrm{~W} / \mathrm{cm}^{2}\right)$, the ions came primarily through the single ionization channel $X_{2} \longrightarrow X^{2+}+X^{+}+3 e^{-}$. Due to the highly anisotropic ionization probability (the geometric alignment), molecules undergoing Coulomb explosion are mostly those aligned vertically 
along the linear polarization of the probe field. Hence, in the absence of the centrifuge, the observed image consisted of a single spot originating from the ions which had zero velocity component in the horizontal plane of the detector (Fig. 4.10 (b)).

Rotational excitation by an optical centrifuge resulted in the transverse component of the ion recoil momentum due to the high angular velocity of the atomic nuclei at the moment of explosion (the drift effect). The energy of this transverse motion reached $\sim 0.86 \mathrm{eV}$ for the highest accessible rotational states and was comparable to the Coulomb explosion energy of a few eV. As a result, the ions spread out horizontally in the plane of molecular rotation, as can be seen in Fig. 4.10 (c). The long axis of the observed ion image (horizontal axis in Fig. 4.10 (c)) can therefore serve as a direct indication of the plane of molecular rotation. As expected, the degree of image elongation grew with the degree of rotational excitation.

In the experiment with $\mathrm{O}_{2}$, the delay between the centrifuge and probe pulses was controlled with a motorized delay line and changed from 0 to 0.9 ns. The final molecular angular momentum was varied by truncating the spectrum of an optical centrifuge as described in section 2.1. The observed ion images are shown in Fig. 4.11 for different degrees of rotational excitation (rows) and at different probe delays (columns).

For all observed angular momenta, as the pump-probe delay increases, the initial plane of molecular rotation splits into three: one which does not precess (horizontal axis in Fig. 4.11) and two others, precessing in clockwise and counter-clockwise direction. The splitting into three planes, clearly visible at longer delays and intermediate $\mathrm{N}$ values (e.g. $\mathrm{N}=61$ ), results from the dependence of the precession frequency on the projection of the electronic spin on the rotational angular momentum $S_{N}$ (see Eq.(4.7)), which can take the values of 0 and \pm 1 .

It could be seen from the images, that the precession frequency decreases with increasing angular momentum, in agreement with Eq.4.7. The quantitative comparison is shown in Fig. 4.12, where the observed values (blue squares) are plotted together with the theoretical prediction (red dashed line) calculated for the magnetic field strength of $|\mathbf{B}|=316 \mathrm{mT}$, determined from the nitrogen experiments (as explained below). The experimental data are in good agreement with the predicted values, except of the higher angular momentum. We attributed this discrepancy to the poorer performance of the centrifuge at higher $N$ values. As the centrifuge field becomes weaker at higher angular velocities, some molecules are "spilled over" from the centrifuge prior to reaching its terminal rotational frequency.

We repeated the experiment with molecular nitrogen. To observe the 
4.4. Dynamics of super rotors in external magnetic fields

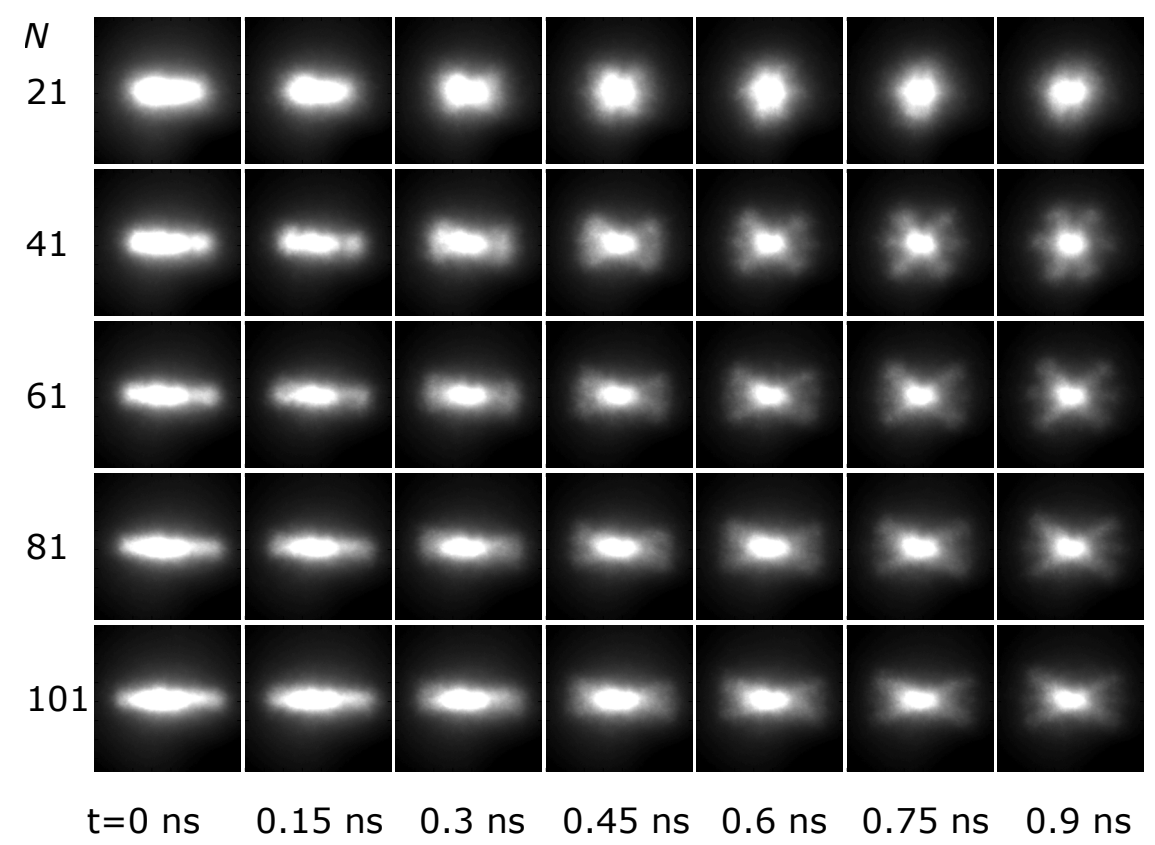

Figure 4.11: Ion images of oxygen super rotors evolving in the external magnetic field. Different rows correspond to different degrees of rotational excitation ( $N$ value on the left), whereas each column corresponds to the evolution time indicated at the bottom. The initial disk distribution splits into three disks precessing with different frequencies according to their spin projections.

magnetic precession of this non-magnetic molecule, probe pulses were delayed for up to $100 \mathrm{~ns}$. The resulting images, taken with the maximum available centrifuge excitation $(N \approx 60)$, are shown in Fig. 4.13 (a). Although long time delays resulted in the reduced image quality, the precession is still clearly seen (notice dashed contour lines indicating counter-clockwise rotation).

To observe this precession at even longer delays, the directional resonanceenhanced multi-photon ionization (directional REMPI) approach was employed [66]. Centrifuged molecules were ionized with a dye laser, resonant with $a^{1} \Pi_{g}\left(v^{\prime}=0, J^{\prime}=31\right) \leftarrow \leftarrow X^{1} \Sigma_{g}^{+}\left(v^{\prime \prime}=0, J^{\prime \prime}=33\right)$ two-photon transition $(285.813 \mathrm{~nm})$, thus probing the population of the $J=33$ rotational level in the ground electronic state. The terminal rotational frequency of the centrifuge field was tuned to populate this state. In a centrifuged ensemble 


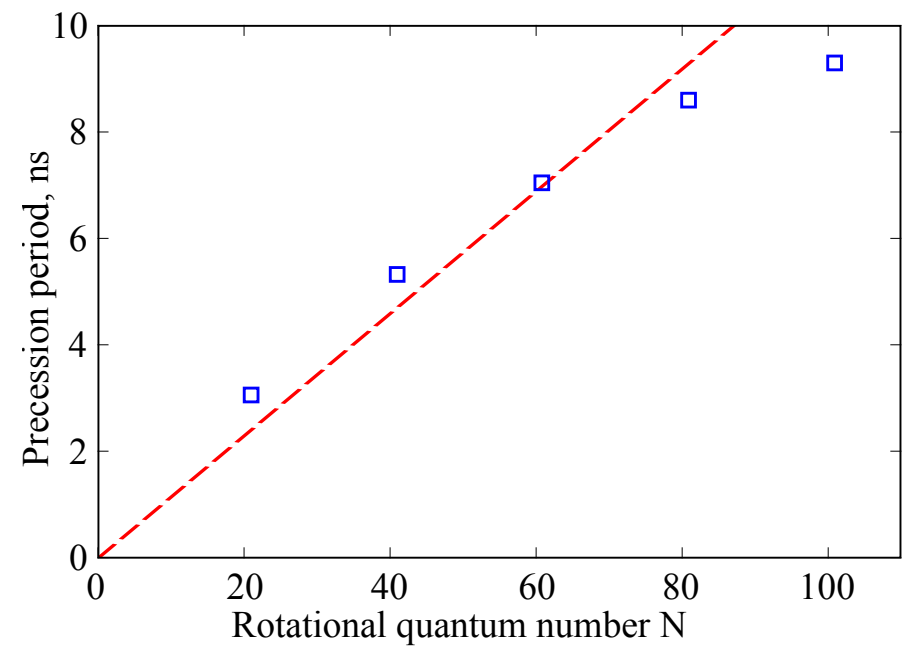

Figure 4.12: Experimentally determined precession period as a function of the rotational quantum number (blue squares) and the expected theoretical dependence (dashed red line).

rotating primarily in one direction, the ionization probability is substantially different when the direction of rotation is the same as, or opposite to, the direction of the circular polarization of the probe light. Namely, when the direction of rotation coincides with that of the probe polarization, the twophoton transition is forbidden by the conservation of the angular momentum projection. Indeed, in this case, the absorption of two photons should increase the magnetic quantum number by two to $J^{\prime \prime}+2$. This value is higher than the highest possible magnetic quantum number for a given angular momentum $M_{\max }^{\prime} \equiv J^{\prime}=J^{\prime \prime}-2$, making this process impossible. In the opposite case, i.e. for the molecules rotating against the probe polarization, the two-photon transition is allowed and results in strong ion yield.

Directional REMPI signal was measured as a function of the probe delay. At each time delay, the probe beam was moved downstream from the pump to account for the supersonic drift of the molecular cloud, which becomes relevant on these time scales. To compensate for the spread of the moving molecular cloud and the change in the beams overlap, each intensity was normalized to the signal obtained with vertical probe polarization at that delay time.

The results are shown in Fig. 4.13 (b). When the direction of the cen- 

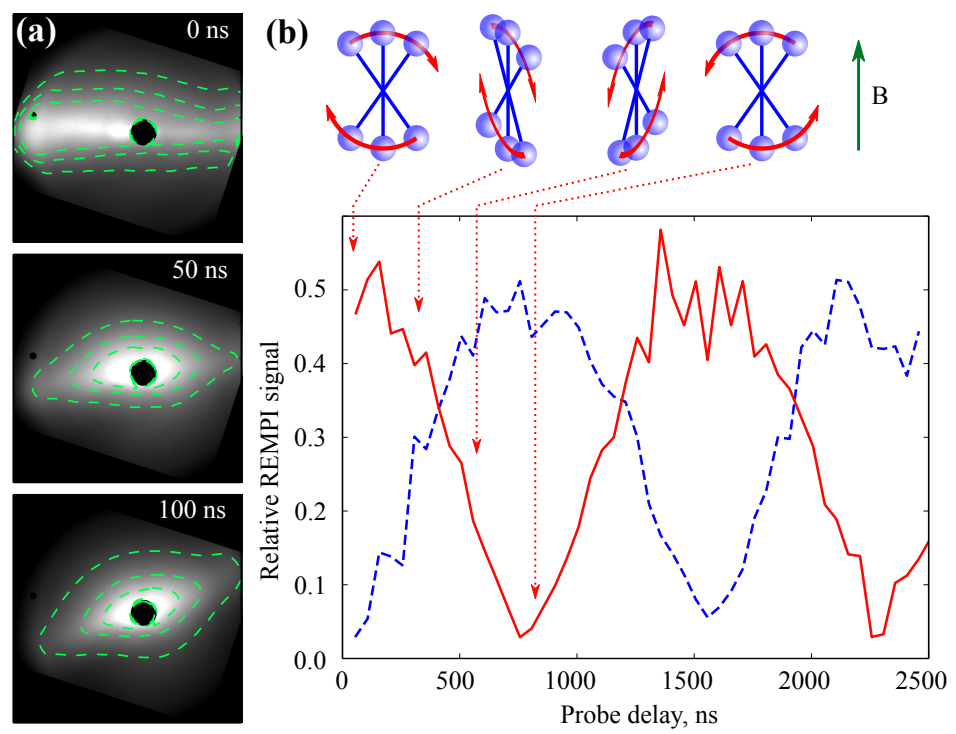

Figure 4.13: (a) Ion images of nitrogen super rotors in the external magnetic field. To improve the visibility of precession, an over-saturated central region is cut off and contour lines (green dashed) are added. (b) Directional REMPI signal as a function of the pump-probe delay. Maximum (minimum) signal is detected when the molecules rotate in the opposite (same) direction with respect to the circularly polarized probe. Solid red and dashed blue lines correspond to the opposite initial directions of molecular rotation, i.e. opposite handedness of the centrifuge pulse.

trifuge spinning was opposite to the probe polarization handedness, the measured signal was initially high (solid red line). However, in half the precession period, when the axis of molecular rotation turned by 180 degrees, the direction of rotation coincided with that of the probe polarization and the signal reached its minimum. These oscillations continued over the whole observed time range, with the plane of polarization completing a total of more than 1.5 full turns over $2500 \mathrm{~ns}$, giving a precession period of $1550 \mathrm{~ns}$. Given the rotational g-factor of $\mathrm{N}_{2}(-0.2681[20])$, this corresponds to the magnetic field strength of $316 \mathrm{mT}$, comparable with the expected value for our magnet. When the centrifuge direction was reversed (dashed blue line in Fig. 4.13 (b)), oscillations of similar period and amplitude, but shifted by $\pi$ radian, were observed in agreement with the expected behavior. 


\section{Chapter 5}

\section{Molecular alignment with an optical centrifuge}

The two commonly used techniques for molecular alignment are the adiabatic and non-adiabatic alignment with intense non-resonant laser pulses 113 , 119. As was discussed in the introduction, the first class is limited to the applications that tolerate the presence of intense aligning laser field, whereas the second one can produce only transient alignment. Very high degrees of rotational excitation became available through the method of an optical centrifuge. However, in contrast to the dynamics of impulsively kicked molecules, the rotation of the centrifuged linear molecules is confined to a plane (section 3.3), rather then a line in the laboratory frame, demonstrating a so-called "antialignment" . Section 5.1 discusses how a modification to the centrifuge allowed us to achieve molecular alignment, similar to that induced by a femtosecond kick, simultaneously with extreme levels of rotational excitation characteristic of the centrifuge spinning.

Another approach to break the planar anisotropy of the centrifuged linear molecules involves the use of static external fields. In section 5.2 we show that strong planar confinement of paramagnetic molecular super rotors can be converted into an anisotropic angular distribution by means of an applied magnetic field. The effect is based on spin-rotational coupling, already discussed in section 4.4, and can be used for fast switching of optical birefringence in gases. Such magneto-rotational birefringence, investigated here, also offers a way of exploring the effect of collisions on the directionality of molecular rotation in dense media.

In the case of asymmetric top molecules, the adiabatic approach is even more desirable. In contrast to linear molecules, asymmetric tops have complicated rotational spectrum which leads to very complicated aperiodic dynamics. In section 5.3, we demonstrate a new mechanism of field-free alignment of asymmetric top molecules with an optical centrifuge. Owing to the adiabaticity of the centrifuge spinning, $\mathrm{SO}_{2}$ super rotors are left rotating with their O-S-O plane aligned with the plane of rotation long after the excitation pulse is gone. Remarkably, this planar alignment reduces complex 
rotational dynamics of an asymmetric top to that of a simple linear rotor.

\subsection{Two-dimensional optical centrifuge}

As was discussed in the introduction (section 1.3), rotational excitation by an optical centrifuge may be thought of as a series of adiabatic Raman transitions between the states with increasing rotational quantum number, $\Delta J=2$ [131, and its projection onto the laser propagation axis $\Delta M_{J}=2$. If the two chirped pulses, used to produce the centrifuge field, are linearly polarized along the same direction, the same reasoning can be applied to show that the molecule under such excitation would still climb the ladder of rotational levels, changing its angular momentum by $\Delta J=2$ at each step, but keeping the projection $M_{J}$ constant.The latter feature stems from the selection rules for a linearly polarized field, $\Delta M_{J}=0$ along the field polarization. This excitation scheme, which we called 2D centrifuge (as opposed to a traditional 3D centrifuge, where the meaning of the "dimensionality" will be clear on the next page) will therefore bring a linear molecule, initially in the ground rotational state, to a highly excited state $\left|J, M_{J}=0\right\rangle$. Since the symmetry between positive and negative values of $M_{J}$ is not broken (unlike the case of a 3D centrifuge) the directionality of the induced rotation is lost. In return, however, we gained the ability to fix the direction of the molecular alignment along the (now constant, as opposed to rotating) field polarization.

The field of a 2D centrifuge, shown in Fig. 5.1(a), was created by passing a $3 \mathrm{D}$ centrifuge beam through a linear polarizer. It consists of a series of pulses, all linearly polarized along the same direction. Both the individual pulse width and the time interval between the pulses are gradually decreasing from the head to the tail of the sequence.

To illustrate and compare the effects of the two centrifuge geometries, $2 \mathrm{D}$ and $3 \mathrm{D}$, on the molecular alignment, we plot the wave function of a rigid rotor $|N=20\rangle$ with $M_{N}=N$ for the $3 \mathrm{D}$ and $M_{N}=0$ for the $2 \mathrm{D}$ centrifuge. The relatively low rotational angular momentum of the final state was chosen here for illustration purposes. Although we routinely spun nitrogen to $N>70$, the calculated distributions, shown in Fig. 5.1 (b,c), become extremely narrow at higher values of $N$. A $2 \mathrm{D}$ centrifuge is seen to produce a well-aligned ensemble, shown in panel (a), whereas the effect of a $3 \mathrm{D}$ centrifuge is very different: molecules are confined in the plane of their rotation, but exhibit no preferential alignment axis, as seen in panel (b).

The induced molecular rotation was analyzed by means of coherent Ra- 


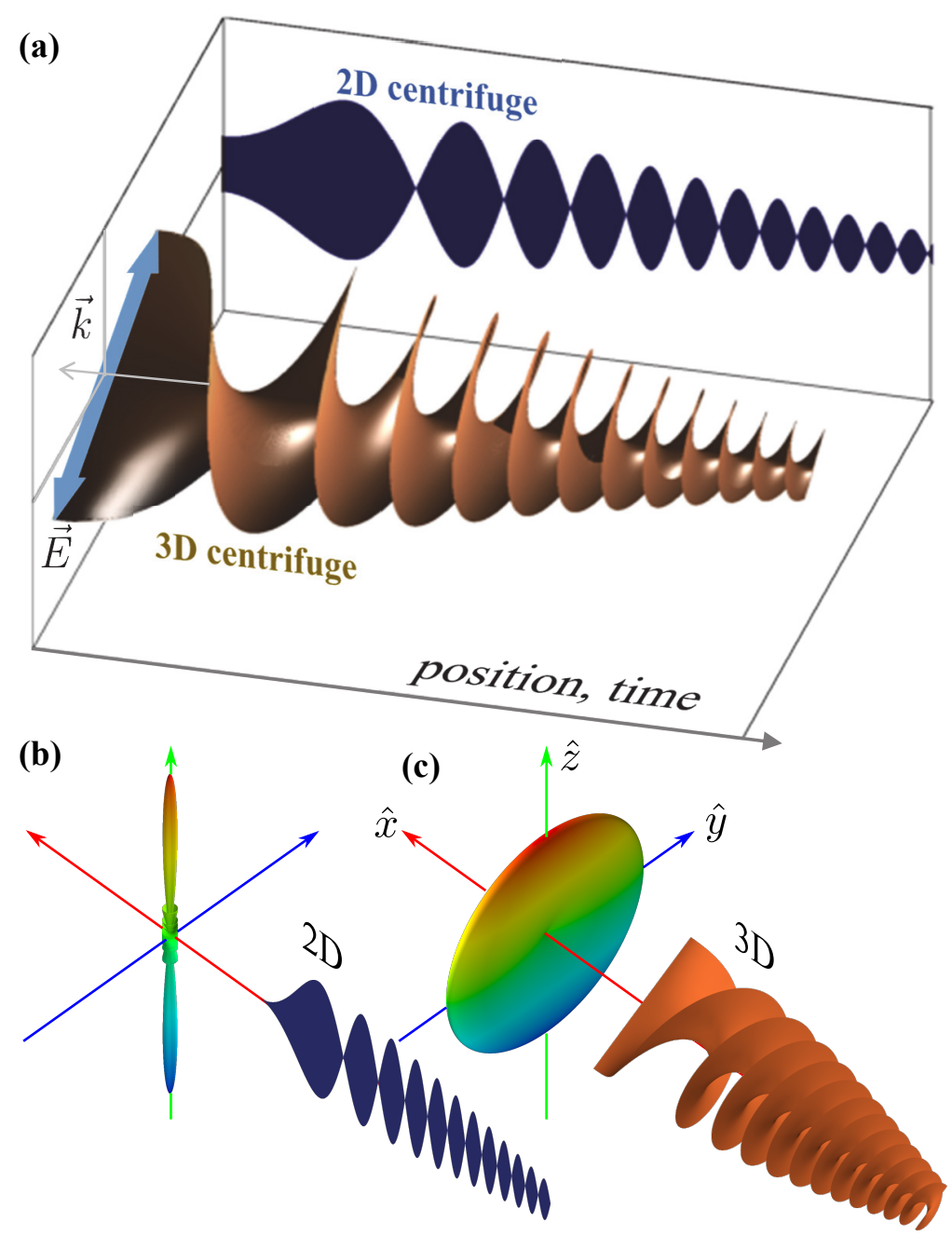

Figure 5.1: (a) Illustration of the concept of a "two-dimensional (2D) centrifuge". The three-dimensional corkscrew-shaped surface represents the field of a conventional "3D centrifuge", propagating from right to left. Shown in blue is the field of a $2 \mathrm{D}$ centrifuge, created by passing the $3 \mathrm{D}$ centrifuge through a linear polarizer. (b, c) Simulated molecular distribution after the rotational excitation by a $2 \mathrm{D}(\mathbf{b})$ and a $3 \mathrm{D}(\mathbf{c})$ optical centrifuge, propagating along the $\hat{x}$-axis.

man spectroscopy (similar to section 3.1). Oxygen molecules in the gas chamber were rotationally excited by either a single femtosecond pulse (here- 
after referred to as a "1D kick"), a conventional (3D) centrifuge or its twodimensional projection ( $2 \mathrm{D}$ centrifuge). The excitation light was focused to a spot size of $90 \mu \mathrm{m}$ (FWHM diameter). For a 1D kick we used a 60 fs pulse, whose total energy of $630 \mu \mathrm{J}$ resulted in the peak intensity of $5.4 \times 10^{13}$ $\mathrm{W} / \mathrm{cm}^{2}$, close to the ionization threshold of oxygen. Probe pulses were 4 ps long $\left(3.75 \mathrm{~cm}^{-1}\right.$ spectral bandwidth (FWHM) centered around $\left.400 \mathrm{~nm}\right)$.

As in our previous Raman experiments, we sent the pump and probe beams collinearly and recorded the Raman spectrum of the probe with a spectrometer. Raman spectrum of oxygen molecules exposed to a single intense femtosecond kick is plotted at the top of Fig.5.2(a). The unshifted Rayleigh peak at $0 \mathrm{THz}$ is surrounded by a series of lines indicative of the laser-induced coherent rotation, with each line corresponding to an individual $|J\rangle \rightarrow|J+2\rangle$ (Stokes) or $|J+2\rangle \rightarrow|J\rangle$ (anti-Stokes) Raman transition. The amplitude envelope of these Raman lines reflects the initial thermal rotational distribution, shifted toward higher rotational levels by $\Delta J \approx 15$, in agreement with the calculated amount of the angular momentum, transferred to the molecule by a laser pulse with intensity $5.4 \times 10^{13}$ $\mathrm{W} / \mathrm{cm}^{2}$.

The result of applying a conventional (3D) optical centrifuge, with its total frequency bandwidth of about $20 \mathrm{THz}$, is shown by the blue plot at the bottom of Fig. $5.2(\mathbf{a})$. Aside from the low-frequency thermal envelopes, corresponding to the molecules too hot to follow the adiabatic spinning [69], the spectrum contains the response from the molecules centrifuged to the angular frequencies between 7.5 and $10 \mathrm{THz}$. Unlike Raman scattering of a probe light with circular polarization, discussed in section 2.2, linearly polarized probe scatters into both Stokes and anti-Stokes Raman sidebands (slightly asymmetric due to the residual ellipticity of the probe polarization).

Applying the 2D centrifuge resulted in the Raman spectra shown in the middle of Fig. $5.2(\mathbf{a})$. Even though the high-frequency Raman sidebands are somewhat lower than in the case of a 3D excitation (compare the lower red and blue curves), the 2D centrifuge was clearly producing molecular super rotors, spinning as fast as $9 \mathrm{THz}$ (remember that the corresponding Raman shift is twice that value). The appearance of a localized (in frequency) wave packet, well separated from the thermal envelope, indicated the adiabatic nature of the rotational excitation. Similar to its $3 \mathrm{D}$ prototype, the 2D centrifuge offers high degree of control over the frequency of molecular rotation. We illustrated this by truncating the spectral bandwidth of the pulses around $8 \mathrm{THz}$, thus moving the centrifuged rotational wave packet to lower frequencies, as shown by the green plot in Fig. $5.2(\mathbf{a})$.

In contrast to the uni-directional rotation of a $3 \mathrm{D}$ centrifuge, the $2 \mathrm{D}$ 


\subsection{Two-dimensional optical centrifuge}
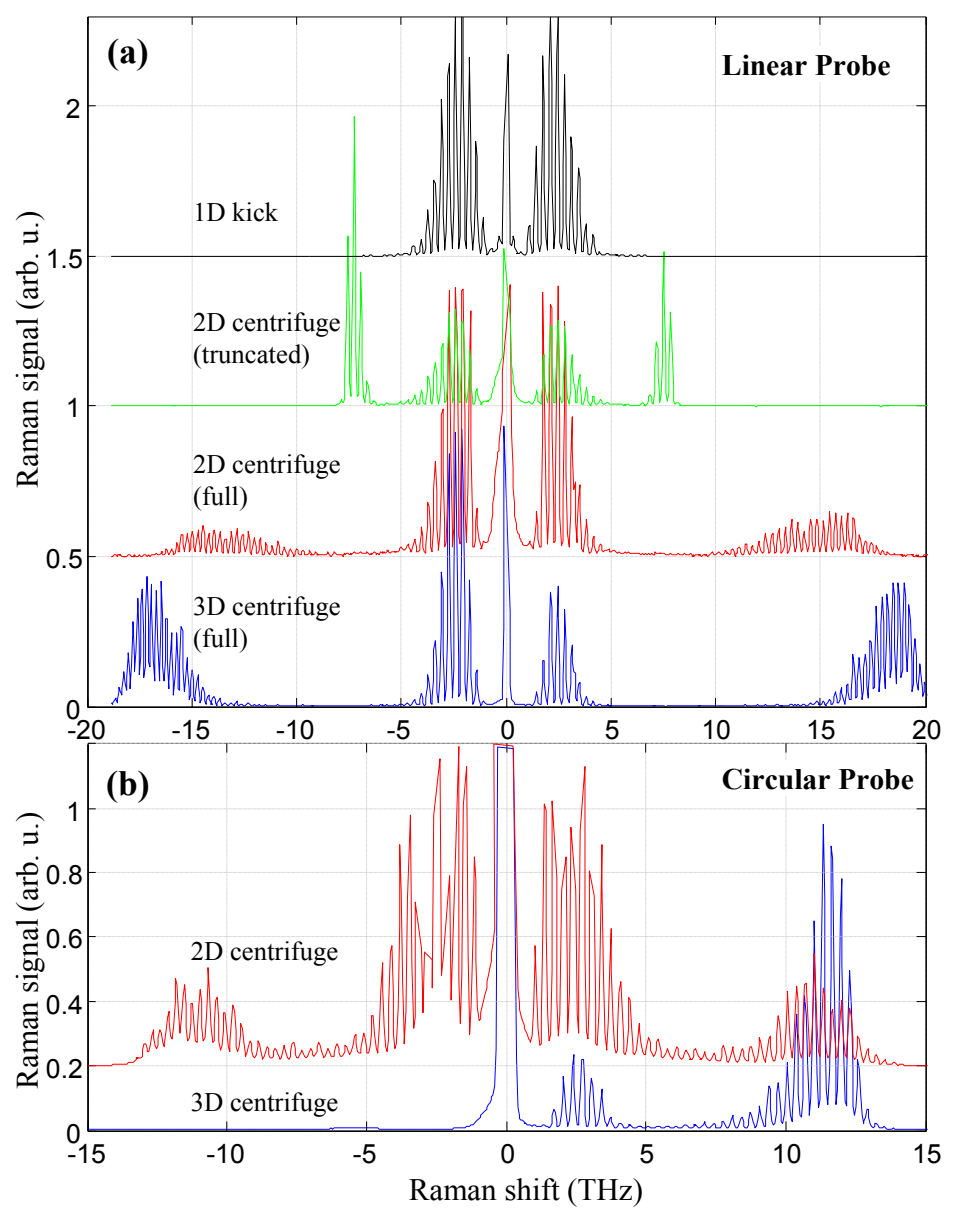

Figure 5.2: (a) Rotational Raman spectra of the centrifuged oxygen molecules recorded with a linearly polarized probe light. From top to bottom, the spectra correspond to the excitation by a single femtosecond pulse (black), a "truncated" 2D centrifuge (green), a full 2D centrifuge (red), and a full 3D centrifuge (blue) with a terminal rotational frequency around 10 THz. The spectra have been recorded at the delay times of 400 ps. (b) Rotational Raman spectra of the centrifuged oxygen molecules recorded with a circularly polarized probe light. Upper red and lower blue curves correspond to the $2 \mathrm{D}$ and $3 \mathrm{D}$ centrifuge, respectively. The spectra have been recorded at the delay times of $400 \mathrm{ps}$. 
excitation field has no preferential sense of rotation. Owing to the selection rule $\Delta M_{J}=0$, the vectors of angular momentum of the molecular super rotors created by a $2 \mathrm{D}$ centrifuge lie in the plane perpendicular to its linear (and non-rotating) polarization, similarly to the effect of a single 1D kick. This lack of directionality is demonstrated in Fig.5.2(b), where we plotted Raman spectra observed with a circularly polarized probe. While the missing Stokes lines in the lower (blue) spectrum indicate that the 3Dcentrifuged molecules rotate in the direction opposite to the circular probe polarization, the presence of both Stokes and anti-Stokes Raman peaks on the upper (red) plot confirms no preferential sense of rotation induced by the $2 \mathrm{D}$ centrifuge.

(a)

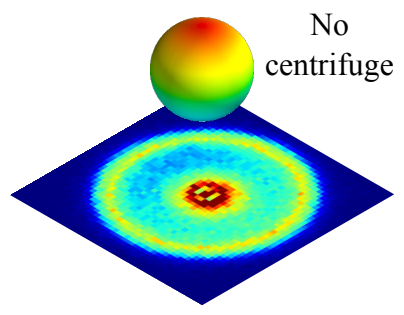

(b)

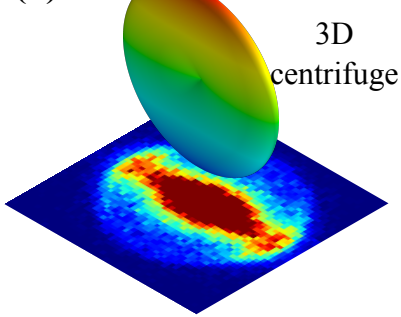

(c)

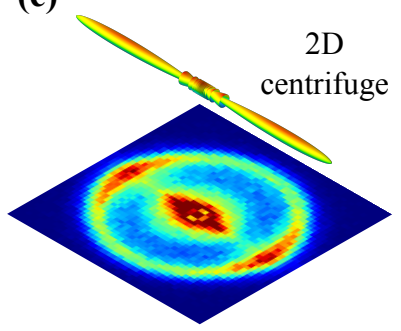

Figure 5.3: Ion images of nitrogen molecules prior to any rotational excitation (a), and following the excitation by a 3D (b) and a 2D (c) centrifuge. Calculated molecular distributions are shown above the respective images.

To determine the degree of molecular alignment produced by a $2 \mathrm{D}$ optical centrifuge, we employed the method of velocity-map imaging (VMI), similar to the setup used in section 3.3. A supersonic expansion cooled the nitrogen gas down to the rotational temperature of $\sim 10 \mathrm{~K}$, with most molecules occupying the rotational ground state. An intense 35 fs laser pulse, linearly polarized along the TOF axis, Coulomb-exploded the molecules into the ion fragments. For the peak intensities of $<10^{15} \mathrm{~W} / \mathrm{cm}^{2}$, used in this study, the main source of anisotropy in Coulomb explosion of $\mathrm{N}_{2}$ stems from the geometric alignment effect [93, with the fragment ions recoiling in the direction of the internuclear axis prior to ionization. The ions, extracted with a DC electric field along the probe polarization, were imaged on a phosphor screen of a microchannel plate detector. The latter was gated in time to mass-select $\mathrm{N}^{+}$ions, originated predominantly from the $\mathrm{N}_{2}^{2+} \longrightarrow \mathrm{N}^{+}+\mathrm{N}^{+}$dissociation channel. Each image was averaged over 500,000 ionization events.

With the absence of rotational excitation, the observed images were cir- 
cularly symmetric, in accordance with the symmetry of the initial ensemble, as shown in Fig. 5.3 (a). Applying either 3D or 2D centrifuge resulted in anisotropic VMI images, plotted in Fig. $5.3(\mathbf{b}, \mathbf{c})$, respectively, reflecting highly anisotropic angular distributions of the centrifuged molecules.

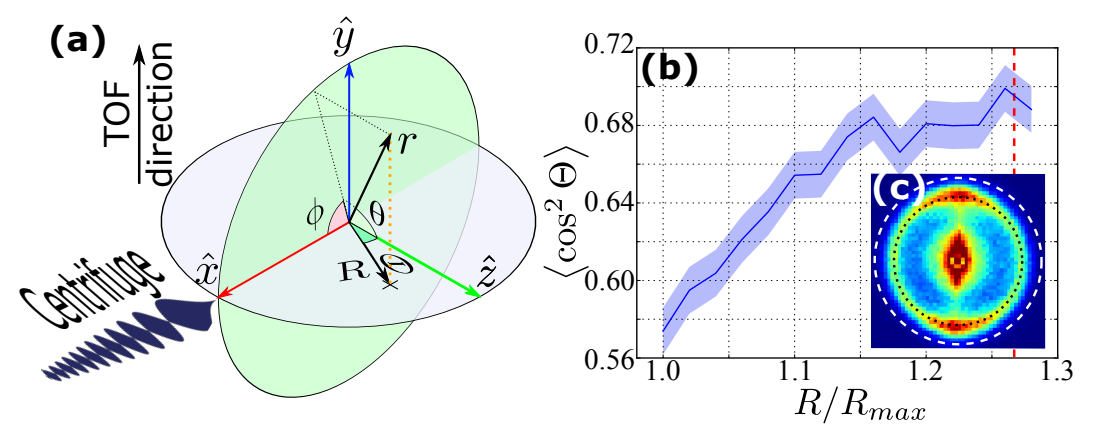

Figure 5.4: (a) Definition of the spherical coordinates $(r, \theta, \phi)$ and polar coordinates $(R, \Theta)$, used to describe the full molecular distribution and its two-dimensional projection on the $x z$ plane of the ion detector. (b) $R$ dependence of the extracted alignment factor, with the shaded region indicating the experimental error. (c) Ion image of $\mathrm{N}_{2}$ molecules aligned with a 2D optical centrifuge. The black dotted and white dashed lines show the circular cross-sections at the radius of a maximum ion signal, $R=R_{\max }$, and the bigger radius used for estimating the true two-dimensional alignment factor, respectively. The latter is also marked by the red dashed line in plot (b).

Since the molecular distribution $f(r, \theta, \phi)$, produced by a 2D centrifuge, is axially symmetric, its two-dimensional cross-section $f(r, \theta, 0)$, containing the axis of symmetry, completely characterizes the degree of the induced alignment (see Fig. 5.4(a) for the definition of the relevant spherical and polar coordinates). In this case, the alignment factor is commonly expressed as $\left\langle\cos ^{2} \theta_{2 D}\right\rangle=\int \cos ^{2}(\theta) f\left(r_{0}, \theta, 0\right) \mathrm{d} \theta$, where $r_{0}=v_{0} t$ is the radial distance covered by the ion of interest, released from the Coulomb explosion with velocity $v_{0}$, during the time of flight $t$.

The experimentally recorded ion images provided us with the projection $F(R, \Theta)$ of the molecular distribution $f(r, \theta, \phi)$ onto the $x z$ plane. As discussed earlier, two distributions are related to one another through the 
well-known Abel transform[128]:

$$
F(R, \Theta)=\int_{R}^{+\infty} \frac{r f(r, \theta, \phi)}{\sqrt{r^{2}-R^{2}}} \mathrm{~d} r,
$$

with $\theta=\arccos \frac{R \cos \Theta}{r}$ and $\phi=\arccos \frac{\tan \Theta}{\tan \theta}$. We note that if the velocity distribution falls off quickly away from its average value $v_{0}$ and if $R$ is sufficiently large, the integral in Eq. (5.1) will be accumulated mostly around its lower limit $r \approx R$. In this case, the observed angular distribution $F(R, \Theta)$ becomes proportional to $f(R, \Theta, 0)$, and the true two-dimensional alignment factor can be approximated as $\left\langle\cos ^{2} \theta_{2 D}\right\rangle \approx\left\langle\cos ^{2} \Theta\right\rangle$. Following this argument, we extracted the angular distributions at various radii and calculated the corresponding values of $\left\langle\cos ^{2} \Theta\right\rangle$ as a function of $R$. As shown in Fig. 5.4(b), the latter indeed saturates at large $R$ 's [white dashed circle in Fig. 5.4(c)], reaching the value of 0.69 .

The retrieved two-dimensional alignment factor of 0.69 is still considerably lower than $\left\langle\cos ^{2} \theta_{2 D}\right\rangle=\int Y_{J}^{0^{*}}(\theta, 0) \cos ^{2} \theta Y_{J}^{0}(\theta, 0) \mathrm{d} \theta=0.87$, anticipated for the rotational quantum state with $J=85$, accessed by our centrifuge. We attribute this disagreement to the following two reasons. First, as $R$ increases, dissociation channels other than $\mathrm{N}_{2}^{2+} \longrightarrow \mathrm{N}^{+}+\mathrm{N}^{+}$may provide a non-negligible contribution to the number of $N^{+}$ions, smearing the angular distribution and lowering the extracted alignment factor.

Another important correction to our estimate of $\left\langle\cos ^{2} \theta_{2 D}\right\rangle$ stems from the drift effect owing to the high rotational frequencies of molecular super rotors $[70$. The rotational energy of the centrifuged nitrogen, studied here, reached $1.8 \mathrm{eV} /$ molecule, becoming comparable with the Coulomb explosion energy release of $6.6 \mathrm{eV} /$ molecule [48]. As a result, the recoil velocities of the fragment ions deviated from the internuclear axis of the exploding molecule and the measured velocity distribution no longer represented the molecular angular distribution.

It should be emphasized, that although the angular distribution acquires an extremely narrow spike-like shape (Fig. 5.3 (c)) at high values of $J$, the $3 \mathrm{D}$ alignment factor $\left\langle\cos ^{2} \theta\right\rangle=\int_{0}^{2 \pi} d \phi \int_{0}^{\pi} \sin \theta d \theta\left|Y_{J}^{0}(\theta, \phi)\right|^{2} \cos ^{2} \theta$ will not exceed a modest factor of 0.5 . This can be understood in the following way. Molecules in states $\left|J, M_{J}^{x}=J\right\rangle^{4}$ can be thought of as rotating around a definite axis, $x$, while being localized in the plane $y z$. The molecules are uniformly distributed along the circle in this plane, and, therefore, their alignment factor along the $z$-axis is given by 0.5 . States $\left|J, M_{J}^{z}=0\right\rangle$ all

\footnotetext{
${ }^{4}$ Note the new quantization axis, $x$.
} 
have their angular momentum lying in plane $x y$, and can be thought of as $\left|J, M_{J}^{x}=J\right\rangle$ "smeared" by the rotation around $z$. This rotation, however does not change the angle between the molecular axes and $z$ direction, and therefore, keeps the alignment factor at 0.5.

\subsection{Magneto-optical properties of paramagnetic super rotors}

In the previous section we showed how the strong centrifuge-induced antialignment can be transformed to the axial alignment by, effectively, smearing the distribution around the polarization axis. A somewhat similar smearing effect, induced by the magnetic field, was demonstrated with oxygen molecules in section 4.4, where spin-rotation coupling mediated the interaction between the external magnetic field and the nuclear motion, leading to fast precession around the magnetic field direction. Due to the dependence of the precession frequency on the projection of the electronic spin on the angular momentum vector, the initial disk distribution was split into three disks, converting the planar confinement $\left(\left\langle\cos \theta_{y, z}^{2}\right\rangle=0.5\right)$ of paramagnetic molecular super rotors into an aligned anisotropic angular distribution $\left(\left\langle\cos \theta_{y}^{2}\right\rangle<0.5,\left\langle\cos \theta_{z}^{2}\right\rangle=0.5\right)$. Here, $\theta_{\alpha}$ is the angle between the molecular axis and unit vector $\hat{\alpha}$, the molecules initially rotate in the $y z$ plane, the field is along $z$, and $\langle.$.$\rangle represents ensemble averaging. In this section, we$ show our ability to use this effect for controlling an optical birefringence of gases with an optical centrifuge in the presence of an external magnetic field.

The experimental system, based on the modified Raman setup (section 2.2), is shown in Fig. 5.5 (a). Both centrifuge and probe beams were focused collinearly between the two Helmholtz coils of a pulsed magnet inside the chamber filled with oxygen at room temperature and variable pressure (Fig. 5.5 (b)). The coils were driven by a $50 \mu$ s current pulse and produced magnetic fields of up to $4 \mathrm{~T}$, which, on the time scale relevant to this study, could be considered static. After scattering off the centrifuged molecules, the polarization of the probe pulses became elliptical, reflecting linear optical birefringence of the molecular ensemble induced by the combination of the centrifuge and magnetic fields. To characterize this "magneto-rotational" birefringence, we passed probe pulses through a pair of crossed linear polarizers, LP and LA in Fig. 5.5 (a).

In Fig. 5.6, the observed spectrum of probe pulses, passed through the ensemble of centrifuged oxygen, is plotted as a function of the magnetic field 


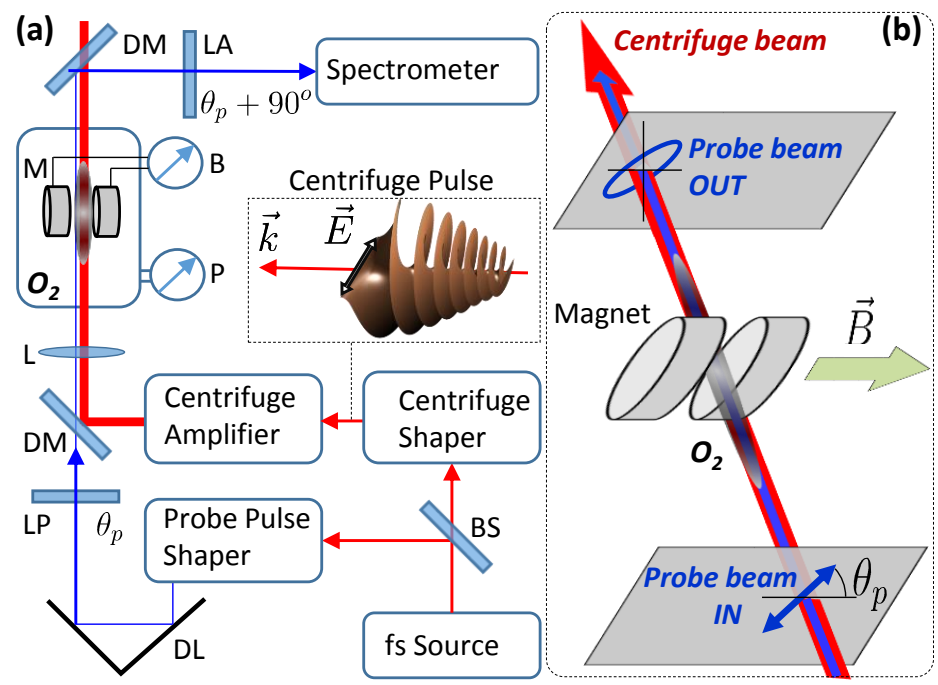

Figure 5.5: (a) Setup for the detection of the "magneto-rotational" birefringence. BS: beam splitter, DM: dichroic mirror, LP (LA): linear polarizer (analyzer) oriented at angle $\theta_{p}\left(\theta_{p}+90^{\circ}\right)$ with respect to $\vec{B}$, DL: delay line, L: lens, M: two magnetic coils connected in a Helmholtz configuration. ' $\mathrm{O}_{2}$ ' marks the pressure chamber filled with oxygen gas under pressure $P$ at room temperature. An optical centrifuge field is illustrated above the centrifuge shaper with $\vec{k}$ being the propagation direction and $\vec{E}$ the vector of linear polarization undergoing an accelerated rotation. (b) Geometry of the magnetic and optical fields used in this work. The cloud of centrifuged molecules is depicted as a dark ellipse.

strength. At zero field, the probe spectrum consisted of two components: the frequency-unshifted Rayleigh line and the down-shifted (Stokes) Raman peak. The former was a result of the depolarization of probe light due to the non-uniform (and randomly changing from pulse to pulse) distribution of molecular axes in the plane of rotation. The second component reflects coherent molecular rotation with an angular frequency of about $6 \mathrm{THz}$ (half the Raman shift). Oxygen molecules rotating with this frequency occupy rotational quantum states with $N=71$.

When the magnetic field was turned on, the amplitude of the Rayleigh peak became much higher, corresponding to strong incoherent (hence, frequencyunshifted) magneto-rotational birefringence. Unlike the weak initial depolarization which had no preferential axis at zero field, the magnetically induced 


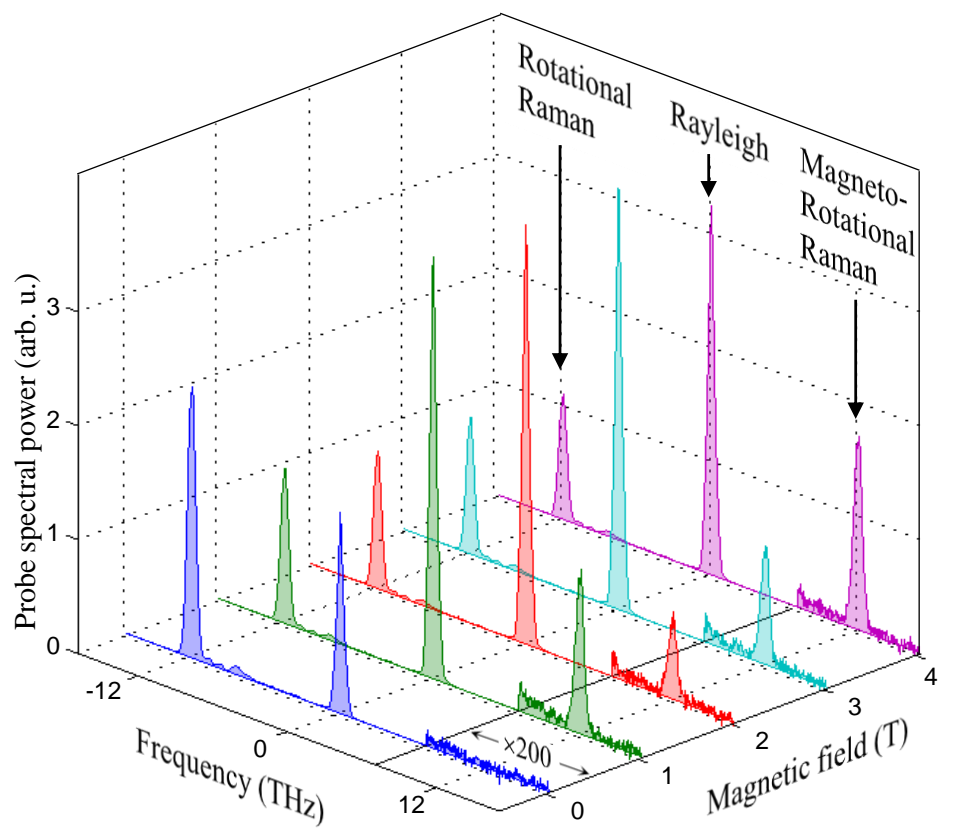

Figure 5.6: Spectrum of probe pulses transmitted through the ensemble of centrifuged oxygen molecules as a function of the applied magnetic field. All spectra have been recorded at the probe delay of $t=1.14$ ns and under $0.3 \mathrm{~atm}$ of gas pressure. Crossed circular (rather than linear) polarizer and analyzer were used here to detect a weak magneto-rotational Raman signal (note the change of vertical scale $(\times 200)$ at frequencies higher than $7 \mathrm{THz}$ ).

birefringence exhibited a well defined optical axis. The applied magnetic field also affected the coherent Raman sidebands. While the initial Raman peak fell off with increasing $B$-field, a weak anti-Stokes Raman line grew on the other side of the spectrum, reflecting molecular rotation in the opposite, with respect to the centrifuge, direction.

In section 4.4, the observed dynamics was described by a simple model, in which the electronic spin, coupled to the molecular angular momentum, was precessing under the effect of magnetic field. To understand the nature of the observed magneto-rotational effects in the gas of paramagnetic super rotors, we calculated the angular distribution of $\mathrm{O}_{2}$ molecules subject to constant magnetic field. As discussed in chapter 4, the interaction between the spins of the two unpaired electrons and the nuclear rotation results in the spin-rotation coupling, which splits each rotational level in three com- 

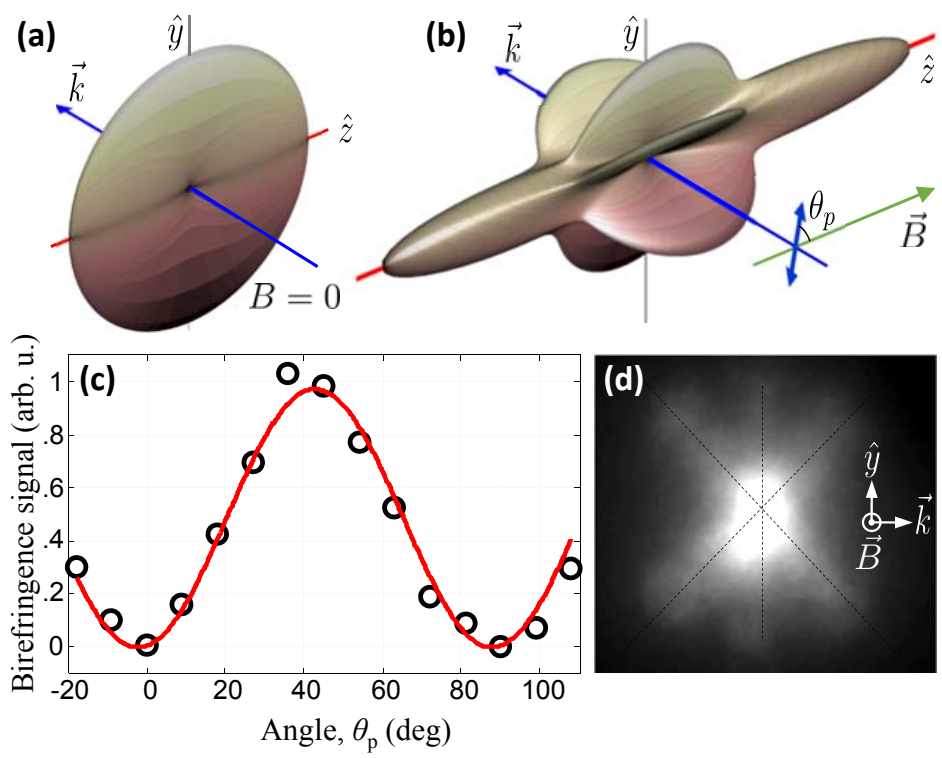

Figure 5.7: (a,b) Calculated angular distribution of the molecular axes for the rotational state with $N=59$ at time $t=0.9 \mathrm{~ns}$ in an external magnetic field of 0 and 0.32 Tesla, respectively. (c) Birefringence signal (scaled to peak at 1) as a function of angle $\theta_{p}$ between the polarization of probe pulses and the magnetic field direction. Black circles: data taken at $2 \mathrm{~T}, t=1.5$ ns, and $N=95$. Red curve is a fit to $\cos ^{2}\left(\theta_{p}\right)$. (d) Experimentally measured distribution of molecular axes, imaged in the direction of the applied field. All parameters are the same as in panel (b).

ponents characterized by the total angular momentum $J=N, N \pm 1$. An applied $B$-field lifts the degeneracy of each level with respect to the projection $M_{J}$ of $\vec{J}$ on the field axis. We calculated the energies $E_{N, J, M_{J}}(B)$ of the magnetic sublevels by numerically diagonalizing the electron-spin Zeeman Hamiltonian, starting from its matrix elements in Hund's case (b) basis set $\left|N, S, J, M_{J}\right\rangle$, with $S=1$ being the total electronic spin.

Given the initial thermal ensemble, the centrifuge creates an incoherent mixture of three states $\left|N, S,(J=N, N \pm 1), M_{J}=J\right\rangle$ with maximum projections $M_{J}$ on the centrifuge direction. Using $J \gg S$, we approximated the angular distribution of the state $\left|N, S, J, M_{J}\right\rangle$ by the spherical harmonics $Y_{J, M_{J}}(\theta, \phi)$. This approximation was verified by a more elaborate calculation of the exact angular distributions [31]. Using this approximation, the final 
angular distribution at time $t$ is given as:

$$
\rho_{N}(\theta, \phi) \approx \sum_{J=N, N \pm 1}\left|\sum_{M_{J}} c_{J, M_{J}} e^{i E_{N, J, M_{J}}(B) t / \hbar} Y_{J, M_{J}}(\theta, \phi)\right|^{2},
$$

where $c_{J, M_{J}}$ are the wave function amplitudes in the coordinate frame with the quantization axis along the applied magnetic field. The results of our calculations for $N=59$ are shown in Fig. 5.7. A disk-like distribution at $B=0$ (panel $(\mathbf{a})$ ) corresponds to the molecular rotation around the centrifuge propagation direction $\vec{k}$ ( $x$ axis), with the molecular axes isotropically distributed in the perpendicular yz plane. As seen in panel (b) of Fig. 5.7. an applied magnetic field splits the disk into three components. This result agrees well with our VMI measurements (section 4.4), reproduced in panel (d) for the same magnetic field and rotational state. In the majority of cases considered here, the coupling between the electronic spin and an ultrafast rotation of super rotors is stronger than its interaction with the external B-field. The perturbative effect of the latter on the precession of the spin around $\vec{N}$ results in a clockwise (counter-clockwise) rotation of $\vec{N}$ around $\vec{B}$ for $J=N-1(J=N+1)$ states and no rotation for $J=N$ states. This leads to an anisotropic distribution stretched along $\hat{z}$. In agreement with this model, an experimentally measured $\cos ^{2}\left(\theta_{p}\right)$ dependence shown in Fig. 5.7 (c) indicates the appearance of a well defined anisotropy axis along the direction of the field, as expected from the geometry of the calculated distribution. We note that for stronger magnetic fields, the three spin states decouple from the molecular angular momentum and the physical picture changes. For example, at the highest magnetic field, achieved with our setup, $B=4 \mathrm{~T}$, the Zeeman energy $\mu_{B} B$ reached $1.9 \mathrm{~cm}^{-1}$ and could not be considered small compared to the spin-rotation interaction, of order of $1 \mathrm{~cm}^{-1}$.

Using the same experimental setup, we analyzed the temporal evolution of the birefringence signal, which we defined as the difference between the amplitude of the Rayleigh peak with and without the applied magnetic field. Fig. 5.8 shows this signal, measured at a fixed magnetic field of 2 Tesla, as a function of the probe time delay with respect to the beginning of the centrifuge pulse. After a rapid initial growth, the magneto-rotational birefringence reached its maximum and then decayed exponentially due to the collisional re-orientation of super rotors.

Truncating the centrifuge pulse in the centrifuge shaper, so as to stop the accelerated rotation at different angular frequencies, allowed us to create rotational wave packets centered at different values of $N$ and study the 


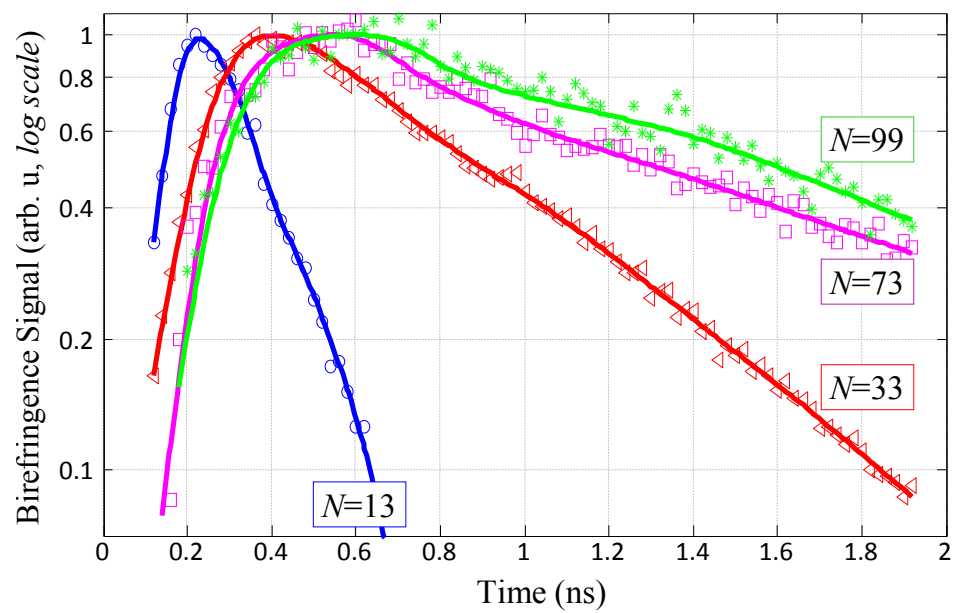

Figure 5.8: Decay of the birefringence signal for different values of the rotational quantum number at $B=2 \mathrm{~T}$. All solid curves are generated by spline-fitting the experimental data (shown with colored markers) and normalized to peak at 1 . The corresponding lifetimes are $85 \pm 10,290 \pm$ $20,660 \pm 50$ and $610 \pm 50 \mathrm{ps} \cdot$ atm for $N=13,33,73$ and 99 , respectively.

dependence of the magnetic effect on the frequency of molecular rotation. The results are plotted in Fig. 5.8 and demonstrate that with the increasing angular momentum, both the growth rate and the decay rate of the induced birefringence became lower. This can be explained by a gyroscopic effect: as the rotational inertia of molecules grew, it became harder and harder to change their angular momenta, either by an external field or by the collisions with other molecules. The effect of high $N$ on the rate of gyroscopic precession of paramagnetic molecules in magnetic field was already explained in section 4.4, In chapter 6, we will discuss in greater detail the effect of collisions on the directionality and the coherence of super rotation.

The dependence of the magneto-rotational birefringence on the strength of the applied magnetic field is shown in Fig. 5.9. After the initial growth with increasing $B$, the signal saturated. For different rotational frequencies, the saturation occurred at different field amplitudes. Slower super rotors did not only require shorter time to respond to the external magnetic field, but also needed weaker fields to reach the plateau. Our numerical estimates of the birefringence signal, based on the calculated angular distributions of molecular axes (see Fig. 5.7), successfully reproduce our experimental findings, showing qualitatively similar behavior. 


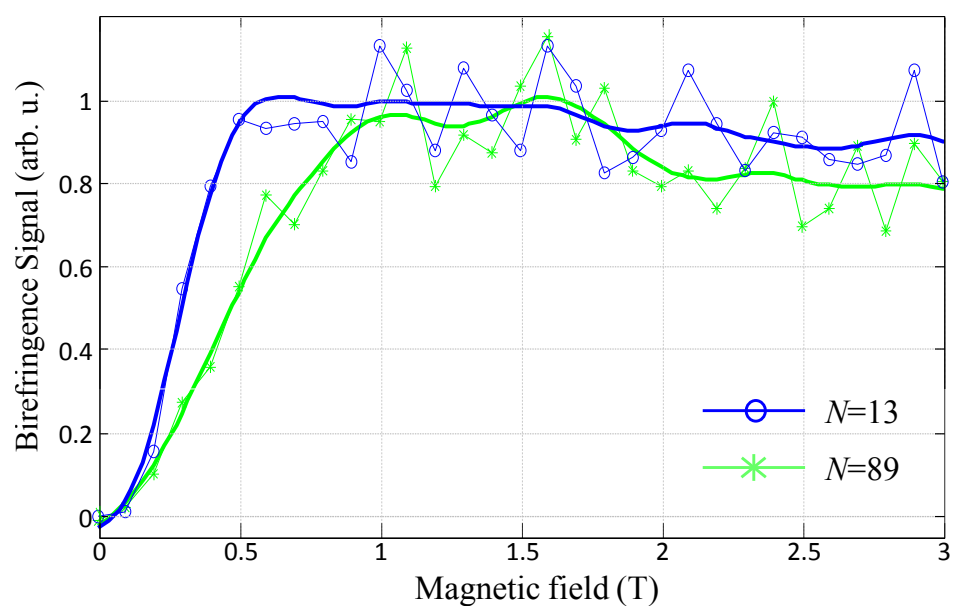

Figure 5.9: Dependence of the birefringence signal recorded at $t=1 \mathrm{~ns}$ on the strength of the applied magnetic field.

Angular distributions are also instructive in the interpretation of the magnetic reversal of the coherent Raman scattering. The latter effect is clearly seen in Fig. 5.6 as the appearance of a weak Raman line with a frequency shift corresponding to the molecular rotation in the direction opposite to the initial, centrifuge-induced rotation. Indeed, the precession of the two parts of the angular distribution around the applied magnetic field, as described earlier in the text, resulted in a small negative component in the projection of the molecular angular momentum on the centrifuge axis.

\subsection{Adiabatic alignment of asymmetric top molecules}

The two previous sections dealt with the axial molecular alignment induced by an optical centrifuge in a gas of linear rotors. In this section we demonstrate that the centrifuge can also be used to achieve a planar alignment of asymmetric top molecules. Our method combines the robustness of an adiabatic alignment with the much desired field-free conditions of the final aligned state. Its essence is illustrated in Fig. 5.10. The leading edge of the centrifuge (left plane) adiabatically aligns the molecular axis of maximum polarizability ( $a$ axis of $\mathrm{SO}_{2}$, parallel to the O-O axis) along the field direction. As the rotational frequency $\Omega$ increases, slowly emerging centrifugal 


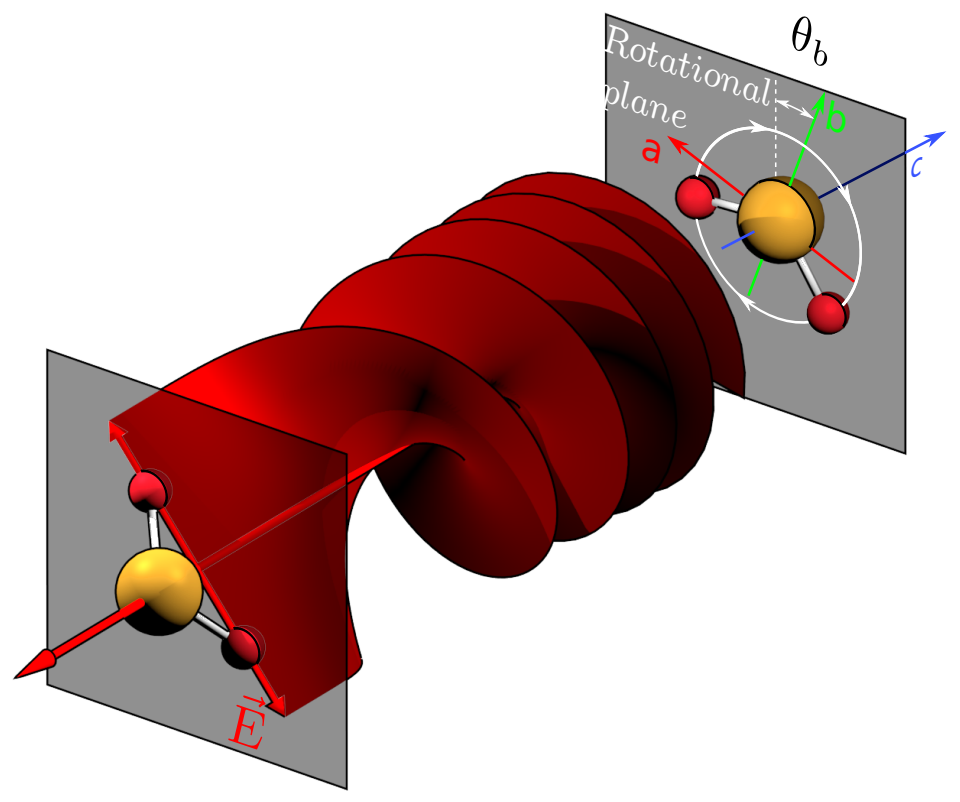

Figure 5.10: Illustration of the main concept of aligning an asymmetric top molecule $\left(\mathrm{SO}_{2}\right)$ with an optical centrifuge. Left side of the centrifuge pulse (red corkscrew surface) corresponds to its leading edge, linearly polarized along $\vec{E}$. Behind the trailing edge of the centrifuge (right side), the molecular plane is aligned in the plane of the induced rotation. The three axes of $\mathrm{SO}_{2}(a, b$ and $c)$ are shown in red, green and blue, respectively. $\theta_{b}$ is the orientation angle measured in this study.

forces in the rotating frame of reference pull the sulfur atom into the plane of rotation, so as to minimize the effective potential $V_{\text {eff }}=-\frac{1}{2} I \Omega^{2}$ by maximizing the molecular moment of inertia $I$ around the rotation axis. For a centrifuged $\mathrm{SO}_{2}$ molecule rotating at $4.5 \times 10^{13} \mathrm{rad} / \mathrm{s}$, the well depth of this centrifugal potential is about $0.9 \mathrm{eV}$. To produce a comparable potential well via the interaction with the molecular polarizability, one would need laser powers of order of $10^{16} \mathrm{~W} / \mathrm{cm}^{2}$, well above the ionization threshold of most molecules.

Observation of the field-free rotational dynamics of the centrifuged $\mathrm{SO}_{2}$ molecules was carried out in the VMI setup described in section 3.3. A supersonic jet of helium-seeded sulfur dioxide was exposed to the field of an optical centrifuge. Rotationally excited molecules were then Coulombexploded by an ultrashort intense probe pulse of either linear or circular 


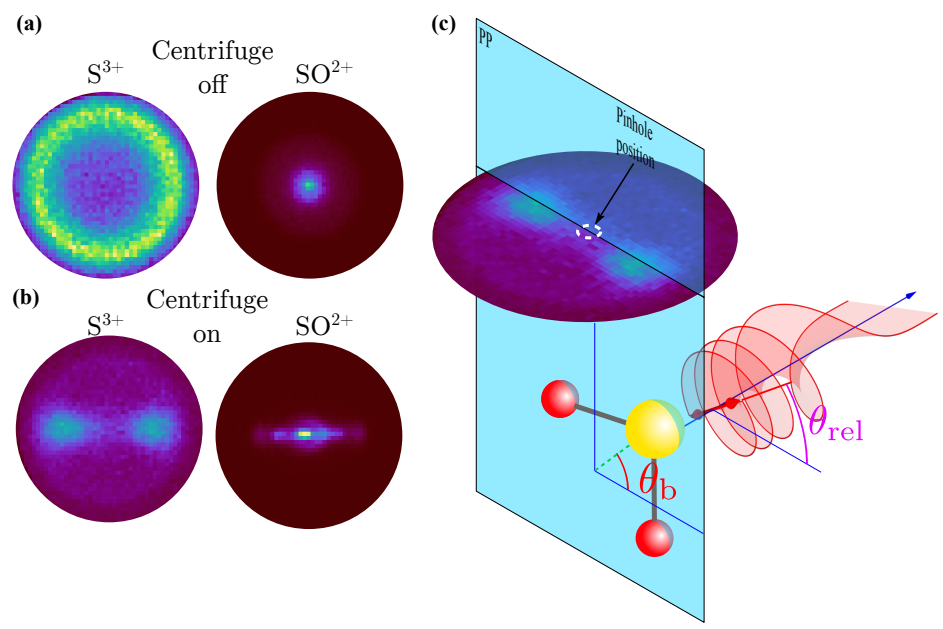

Figure 5.11: (a,b) Images of $S^{3+}$ and $S O^{2+}$ fragments originated from the rotationally cold and centrifuged $\mathrm{SO}_{2}$ molecules, respectively.(c) Setup for the in-plane distribution measurement of the molecular $b$-axis (green dashed line). PP marks the polarization plane of the lasers.

polarization. The momenta of the fragment atomic and molecular ions, bearing the information about the molecular orientation at the moment of explosion, were projected onto the microchannel plate detector with a phosphor screen.

Gating the MCP voltage for the arrival of either $S^{3+}$ or $S^{2+}$ ions, we recorded the corresponding VMI images obtained with linear probe polarization, normal to the plane of the MCP. Due to the symmetry of $\mathrm{SO}_{2}$, the recoil momenta of both species lie in the O-S-O plane of the fragmented molecule and, therefore, define unambiguously the molecular plane at the time of explosion. Without the centrifuge, the observed images exhibited an axial symmetry, expected for the spherically symmetric molecular distribution (Fig. 5.11 (a)). As we turned the centrifuge on, both images collapsed to the plane of the laser-induced rotation, as shown in Fig. 5.11 (b). The simultaneous collapse of the two distributions for both ion fragments indicated strong planar alignment of $\mathrm{SO}_{2}$ molecules.

At high rotational energies, comparable to the kinetic energy release of the Coulomb explosion, extracting the degree of planar alignment from the velocity map images in Fig. 5.11 (c) is complicated by the additional drift of the ion fragments away from the rotationally unperturbed trajectories 


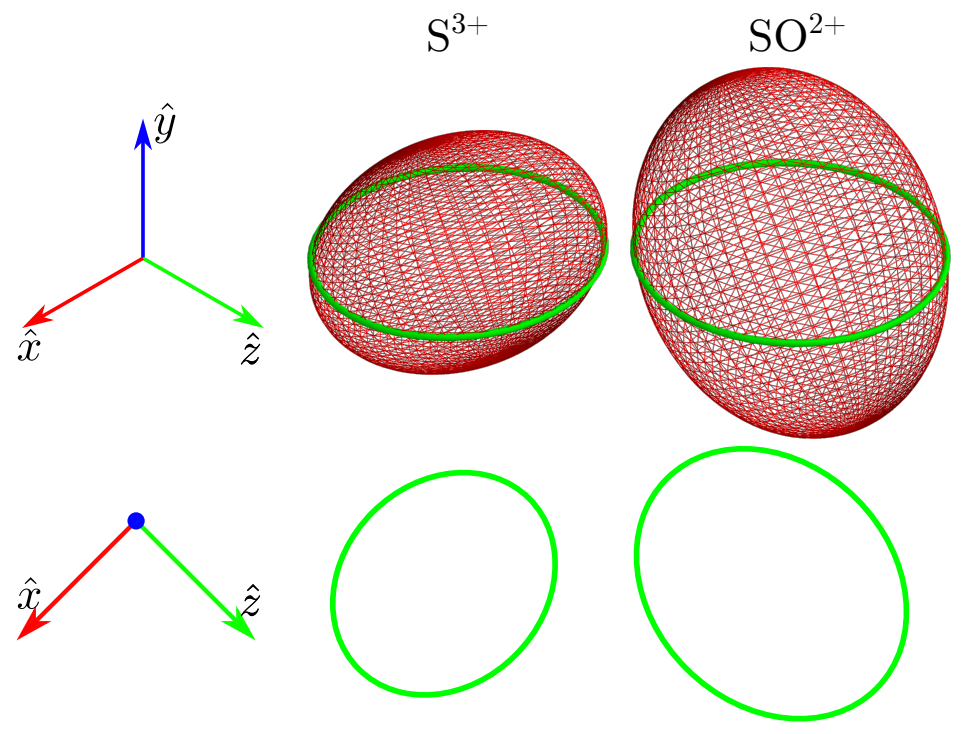

Figure 5.12: Illustration of the effect of underestimated and overestimated alignment of $\mathrm{S}^{3+}$ and $\mathrm{SO}^{2+}$, respectively.

(section 3.3). Ultrafast synchronous rotation of the centrifuged molecules results in tilting the Newton sphere of the fragment velocities with respect to the sphere of possible molecular orientations. In the case of $\mathrm{S}^{3+}$ ions, preferably ejected orthogonally to the laser field polarization[48], the drift effect leads to the compression of the ion image towards the center. On the other hand, the image of $\mathrm{SO}^{2+}$, preferably ejected along the field polarization, is stretched outwards. This amounts to an underestimated planar alignment factor determined from the $\mathrm{S}^{3+}$ image, $\left\langle\cos ^{2} \theta\right\rangle_{2 D}=0.64$, and an overestimated value of 0.90 determined from the $\mathrm{SO}^{2+}$ channel. This effect is illustrated in Fig. 5.12 , where the anisotropic distributions of $\mathrm{S}^{3+}$ and $\mathrm{SO}^{2+}$ recoil velocities are depicted with red ellipsoids, compressed or stretched along the probe polarization direction (axis $y$ ), respectively. The drift effect due to the fast molecular rotation around $x$ axis corresponds to the effective tilting of the distribution ellipsoids around this axis. As a result, the observed distribution along the $z$ axis is either underestimated or overestimated with respect to the $x$ axis, as demonstrated by the compression and expansion of the green ellipses in the bottom row of Fig. 5.12, respectively.

We studied the rotational dynamics of aligned $\mathrm{SO}_{2}$ within the rota- 
tion plane using circularly polarized probe pulses to ensure an isotropic in-plane ionization probability, similarly to the experiments with diatomic super rotors (section 4.2). The in-plane angular distribution of centrifuged molecules was measured as a function of the delay time between the end of the centrifuge pulse and the probe-induced Coulomb explosion. The distribution was determined by recording the ion signal at a single location on the MCP detector as a function of the release angle of the centrifuge, $\theta_{\text {rel }}$ (see Fig. 5.11 (c)). As opposed to $\mathrm{O}_{2}$, the kinetic energy release in the Coulomb explosion of $\mathrm{SO}_{2}$ was distributed over a broader range of energies, causing the circles of the dissociation channels to have a diffuse edge (Fig. 5.11 (a)). For this reason, the signal was measured at the center of the image, rather then at its boundary.

With the MCP voltage gated at the arrival of $S^{3+}$ ions, the detected signal was proportional to the amount of molecules with the orientation angle $\theta_{b}=90^{\circ}$ between the horizontal axis and the molecular $b$ axis (the one bisecting the O-S-O bond angle, Fig. $5.11(\mathbf{c})), f_{\theta_{\text {rel }}}^{b}\left(\theta_{b}=90^{\circ}\right)$. As was shown in section 4.2 , the distribution $f_{\theta_{\text {rel }}}^{b}\left(\theta_{b}=90^{\circ}\right)$ is equivalent to $f_{0}^{b}\left(\theta_{b}=90^{\circ}-\theta_{\text {rel }}\right)$. In what follows, we investigate the latter distribution [hereafter referred to as $f\left(\theta_{\text {rel }}\right)$ ] as a function of the centrifuge-to-probe delay time.

The observed time dependence of $f\left(\theta_{\text {rel }}\right)$ is shown Fig. 5.13 (b). Truncating the centrifuge pulse in time enables us to control the final angular frequency of the centrifuged molecules, which in this case was set to $10^{13} \mathrm{rad} / \mathrm{s}$. Due to the centrifuge-induced planar alignment, the number of the rotational degrees of freedom of $\mathrm{SO}_{2}$ molecules was reduced to one and their dynamics became periodic, similar to the dynamics of a linear rotor. This periodicity, though not apparent from the coarse two-dimensional scan, is clearly evident from the extracted alignment factor, $\beta_{2 D}=\left\langle\cos ^{2} \theta\right\rangle_{2 \mathrm{D}}-\frac{1}{2}$ (with $\langle. .\rangle_{2 \mathrm{D}}$ being the in-plane average and $\theta$ being measured from the axis of maximum, for a given delay, alignment), plotted in Fig. 5.13 (a). Each peak corresponds to the field-free three-dimensional alignment of $\mathrm{SO}_{2}(\mathrm{FF} 3 \mathrm{DA})$. We note, that although the observed two-dimensional alignment factor in the plane of rotation is not much higher than its isotropic value of 0.5 , the strong molecular confinement to this plane results in rather high values of FF3DA. Using the lower and upper bounds of the achieved planar alignment extracted from the two VMI channels (as discussed above), we arrive at the corresponding estimates of $\left\langle\cos ^{2} \theta_{b}\right\rangle$ of 0.40 and 0.49 . These values are comparable with the theoretical predictions for other methods of non-adiabatic three-dimensional alignment[105, 127]. 
The relatively small degree of the observed FF3DA stems from the adiabatic mechanism of the centrifuge spinning, which results in a low number of quantum rotational states in the excited wave packet. As we have shown in section 4.2, a small number of participating states gives rise to long windows of classical-like rotation around the time of each revival, with the rotational frequency equal to the terminal frequency of the centrifuge. The same classical-like rotation was observed here with $\mathrm{SO}_{2}$ molecules when we performed a fine time scan around any of the alignment peaks, as demonstrated in Fig. 5.13 (c).

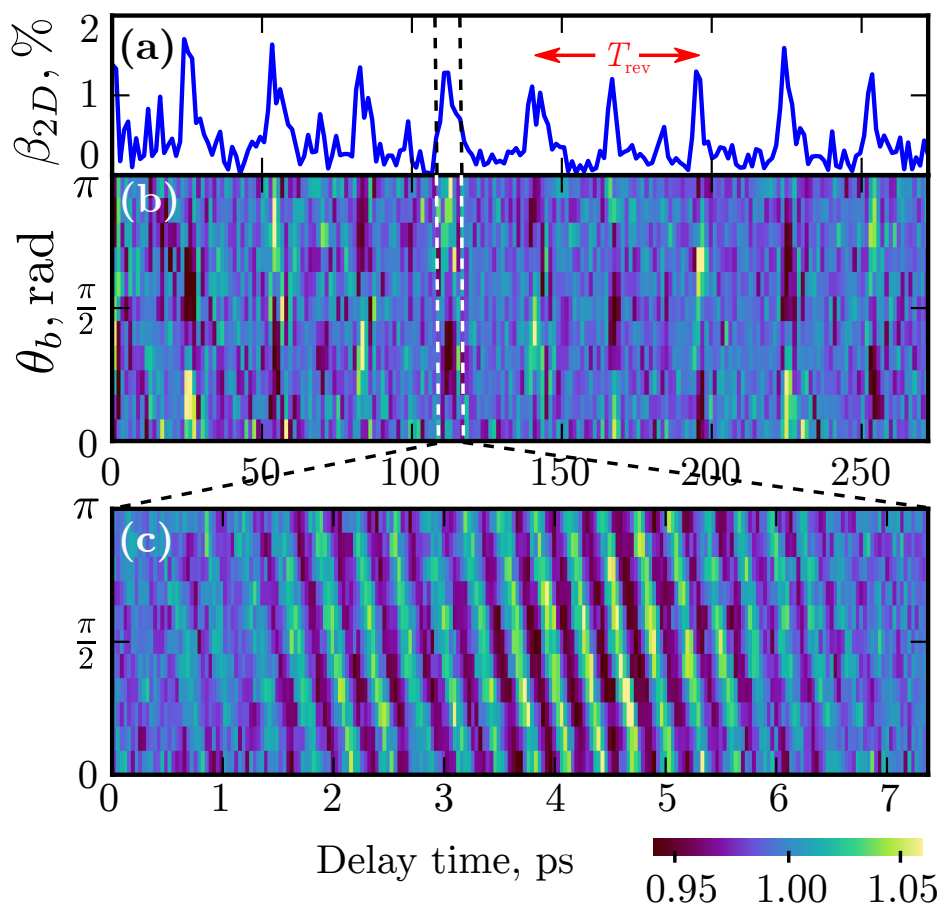

Figure 5.13: (a) Periodic revivals of the calculated two-dimensional alignment factor $\beta_{2 D}=\left\langle\cos ^{2} \theta\right\rangle_{2 D}-\frac{1}{2}$, determined from (b) Time evolution of the molecular in-plane angular distribution. (c) High resolution time scan around the alignment peak.

As expected for the effectively one-dimensional rotation, the alignment peaks are separated by half the revival period $T_{\mathrm{rev}}=2 \pi \hbar\left(d^{2} E / d J^{2}\right)^{-1}$, where $E(J)$ is the rotational energy for a given rotational quantum number $J$ and $\hbar$ is the reduced Plank's constant [5, 111]. Using the rigid rotor's energy 


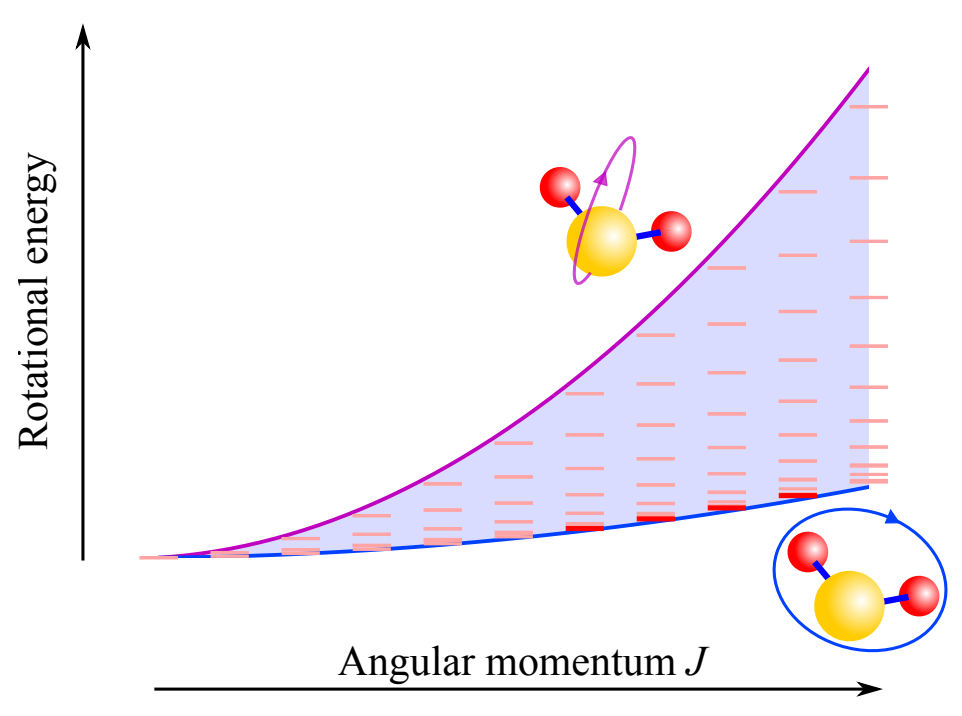

Figure 5.14: Schematic rotational spectrum of asymmetric top molecules.

spectrum $E(J)=\frac{\hbar^{2}}{2 I} J(J+1)$, with $I$ being the molecular moment of inertia, one finds $T_{\mathrm{rev}}=2 \pi I / \hbar$. At lower centrifuge frequencies, the experimentally detected revival period of $57 \mathrm{ps}$ corresponds to $I=58 \AA^{2} \cdot$ amu in good agreement with the known value for the molecule's largest moment of inertia $\left(I_{c}\right)$ around the axis normal to its plane (c-axis) [46].

To better understand the mechanism of revivals, it is useful to first describe the rotational spectrum of an asymmetric top molecule. The spectrum of a classical asymmetric top is shown in Fig. 5.14 by the light blue area. For any angular momentum $J$, the energy of the top can take any value between the values, corresponding to the rotation around "heavy" (bright blue line) and "light" (purple line) axes. In a quantum mechanical system, the angular momentum is quantized and can only take values of integer number of $\hbar$. For each allowed $J$, there are now only $(2 J+1)$ allowed energy levels, shown in Fig. 5.14 by thin red lines, that are enumerated by a new quantum number $\tau$, called the asymmetric top quantum number. For a given $J, \tau$ takes integer values from $-J$ to $J$, with $\tau=-J$ corresponding to the lowest energy state, and $\tau=J$ - to the highest one. Due to the quantum-classical correspondence, the energies of high states $|J, \tau=-J\rangle$ are expected to be close to the classical limit $\epsilon_{J}=C J(J+1)$, corresponding to the blue line. The observed revivals, known as C-type transients[55], can be attributed to the beating between a few such states, populated by the centrifuge and 
labeled with thick red lines in Fig. 5.14.

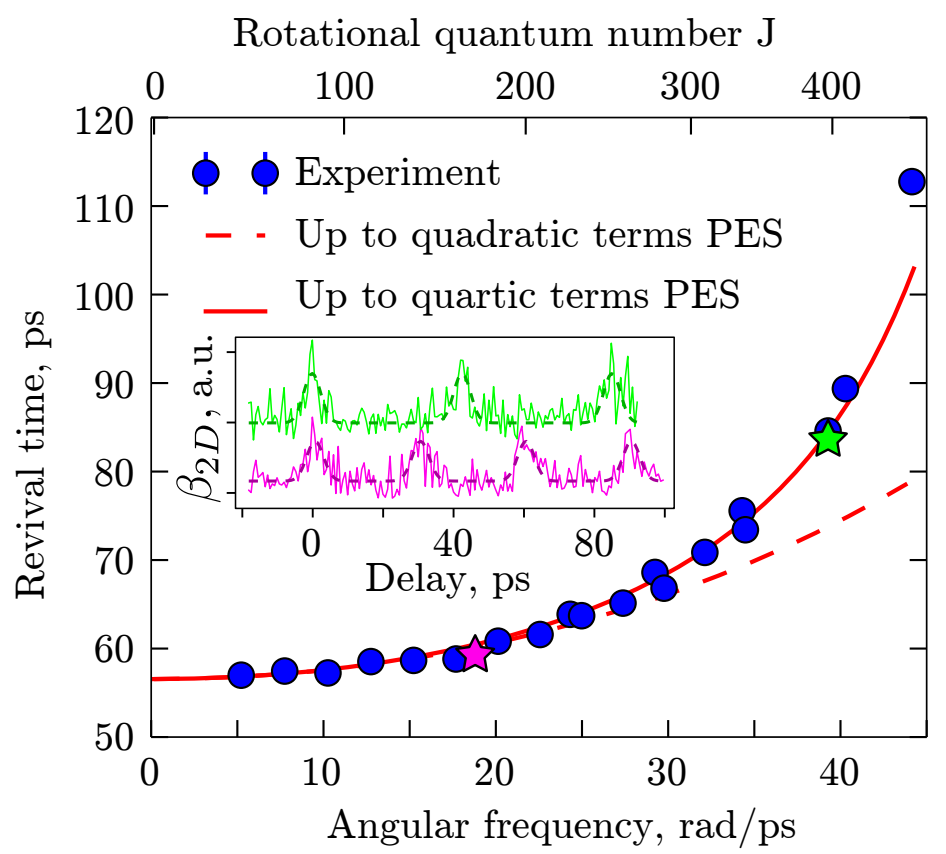

Figure 5.15: Revival period as a function of the rotational frequency of $\mathrm{SO}_{2}$, with the rotational quantum numbers shown along the upper horizontal axis. Experimental data (blue circles) are compared with the results of classical calculations, in which the potential energy surface is expanded to second and fourth order in deformation coordinates (dashed and solid lines, respectively). The inset shows the dependence of the $2 \mathrm{D}$ alignment $\beta_{2 D}$ on the pump-probe delay, used for calculating the revival time at two angular frequencies, $18.8 \mathrm{rad} / \mathrm{ps}$ (magenta) and $39.3 \mathrm{rad} / \mathrm{ps}$ (green), labeled with the correspondingly colored stars in the main plot. Solid lines represent experimental data, while the dashed lines show the fitting functions used to extract the revival period.

At higher angular frequencies, the rising centrifugal forces stretch and bend the molecule, distorting the two S-O bonds and the angle between them. This causes the moment of inertia, and hence the revival period, to increase, as evident from the experimental data in Fig. 5.15 (blue circles). To describe the effect of the centrifugal distortion on the revival period, we carried out the following classical calculations. For a given rotational state 
$J$, we minimized the total energy:

$$
E\left(J, r_{1}, r_{2}, \alpha\right)=\frac{\hbar^{2} J^{2}}{2 I_{c}\left(r_{1}, r_{2}, \alpha\right)}+V\left(r_{1}, r_{2}, \alpha\right)
$$

over the S-O bonds lengths $r_{1,2}$ and O-S-O angle $\alpha$. The potential energy surface (PES) $V$ of $\mathrm{SO}_{2}$ was expanded in Taylor series up to the fourth order in deformations $\delta x_{i}=r_{1}-r_{e}, r_{2}-r_{e}, \alpha-\alpha_{e}$, where $r_{e}$ and $\alpha_{e}$ are the values of $r_{1,2}$ and $\alpha$ for the molecule at rest:

$$
\begin{aligned}
& V\left(r_{1}, r_{2}, \alpha\right)=V\left(r_{e}, r_{e}, \alpha_{e}\right)+\frac{1}{2} \sum_{i j} f_{i j} \delta x_{i} \delta x_{j}+ \\
& \frac{1}{6} \sum_{i j k} f_{i j k} \delta x_{i} \delta x_{j} \delta x_{k}+\frac{1}{24} \sum_{i j k l} f_{i j k l} \delta x_{i} \delta x_{j} \delta x_{k} \delta x_{l},
\end{aligned}
$$

with the corresponding force constants $f$ taken from Ref.88. We then determined the classical angular velocity and the revival period for a given $J$ as

$$
\omega(J)=[E(J+1)-E(J)] / \hbar
$$

and

$$
T_{\mathrm{rev}}=2 \pi \hbar[E(J-1)-2 E(J)+E(J+1)]^{-1},
$$

respectively. The results of these calculations are shown in Fig. 5.15. Expanding PES to second order in deformations $\delta x_{i}$ failed to explain the experimental observations at frequencies higher than $3 \times 10^{13} \mathrm{rad} / \mathrm{s}$ (dashed line). The quartic expansion, on the other hand, proved sufficient (solid line). At the highest achieved rotational frequencies of $4.4 \times 10^{13} \mathrm{rad} / \mathrm{s}$, the calculated bending angle and bond stretching reached $10^{\circ}$ and $10 \mathrm{pm}$, respectively. Both bonds were found to stretch by an equal amount, as could be anticipated from the symmetry of the system. 


\section{Chapter 6}

\section{Collisional decay of rotational excitation in dense media}

Previous chapters concentrated mostly on the implementation of the rotational control in molecules, i.e. the generation and characterization of super rotor states with an optical centrifuge. In this chapter we will discuss how these extreme rotational states decay due to inter-molecular collisions in a dense gas medium.

Recent developments in controlling molecular rotation with nonresonant laser pulses [30, 94 have stimulated active research on the exchange of energy between a rotating molecule and its environment. Transient molecular alignment has been first proposed [102] and later implemented as a powerful probe of collisional relaxation in a series of pioneering experiments [50, 58, 129]. The three relaxation steps, associated with (i) rotational decoherence, (ii) rotational reorientation and (iii) rotation-translation (RT) thermalization, have been identified in the theoretical model[42], yet found to overlap in time too closely to enable an individual experimental study of each process separately[58].

Rotational decoherence in dense gaseous media is important for the fundamental understanding of the dissipative properties of gases, as well as in the practical aspects of thermochemistry and combustion research $[89,90$, 109, 123]. One of the most interesting aspects of the collision-induced rotational decoherence is the question about its dependence on the speed of molecular rotation and temperature. From the very first experimental works on the topic [13, 98], it was suggested that the rate of rotational relaxation should drop with increasing rotational quantum number $J$, i.e. that faster molecular rotors are more robust with respect to collisions. In thermal ensembles, both the rotational energy of the accessible states as well as the collision energy scale equally with temperature. Hence, reaching higher values of $J$ by means of heating up the gas sample does not allow extending 
the study of decoherence rates to a new regime, where the rotational energy exceeds the collisional energy. In section 6.1, we describe how an optical centrifuge allowed us to reach this regime, controlling the angular momentum excitation separately from the gas temperature.

Ultrahigh values of the molecular angular momentum $J$, provided by the centrifuge, together with the propensity of collisions to conserve its orientation [42] resulted in the "gyroscopic stage" [62], which outlived the rotational coherence by a few nanoseconds. We studied the dynamics of this relaxation step by tracking an optical birefringence of the centrifuged gas in the direction perpendicular to the direction of the centrifuge. This transverse birefringence stemmed from a strong permanent confinement of molecular super rotors in the plane of their rotation, already discussed in the previous chapter. In the longitudinal direction along the centrifuge, the change in refractive index owing to the fast unidirectional molecular rotation created a refractive "gyroscopic channel", which is described in section 6.2.

Combining the longevity of the gyroscopic stage with the axial alignment provided by the 2D centrifuge approach, demonstrated in section 5.1, allowed us to create a long lived permanent alignment in the ensembles of diatomic molecules, discussed in section 6.3.

The final stage of rotational relaxation, the RT thermalization, leads to a local heating of the gas followed by the formation of an acoustic wave[15, 59, 65, 106, 141]. Previous studies of rotational photo-acoustics were based on the impulsive excitation of molecular rotation by a single femtosecond laser pulse or a short series of up to four pulses. These methods were limited to relatively low levels of rotational excitation with molecules gaining only a few units of angular momentum. At this limit, the amount of rotational energy deposited by the laser field is rather hard to control. As a result, the direct connection between the rotational energy and the amplitude of the sound wave has not been established beyond the perturbative limit. By utilizing optical centrifuge and a state-resolved Raman detection, we were able to measure the rotational energy of molecules and correlate it with the

independently measured acoustic wave intensity. This study is described in section 6.4.

\subsection{Effects of ultrafast rotation on collisional decoherence}

The decrease of the collisional decoherence rate with the angular momentum $N$ is expected from the intuitive "exponential-gap law" (EGL) according to 
which the decay rate decreases as $\exp \left[-\Delta E_{N} / k_{B} T\right]$ with the increasing distance between the rotational levels $\Delta E_{N}$ (here $k_{B}$ is the Boltzmann constant and $T$ is the temperature of the gas). The refined version of EGL, known as the "energy corrected sudden" (ECS) approximation and introduced by DePristo et al. [22], is a popular model which successfully explained a large number of experimental observations [12, 67, 71, 89, 90, 123].

The ECS theory describes the collisional decay rate in terms of an "adiabaticity parameter" $a \equiv \omega_{N} \tau_{c}=\omega_{N} l_{c} / v_{c}$, where $\omega_{N}$ is the frequency of molecular rotation, $\tau_{\mathrm{c}}$ is the collision time, $l_{c}$ is a characteristic interaction length (usually determined empirically) and $v_{c}$ is the mean relative velocity between the collision partners. Since $a=2 \pi \tau_{\mathrm{c}} / T_{\mathrm{N}}$ (with $T_{\mathrm{N}}$ being the rotation period), it may also be viewed as the angle, by which a molecule rotates during the collision process. When $a \ll \pi$, the collision is sudden and the energy transfer does not depend on $N$ (the non-adiabatic regime). In the case of the finite duration of collisions, i.e. $a \gtrsim \pi$, the ECS model calls for scaling the decay rate with a $N$ - and temperature-dependent correction factor

$$
\Omega_{l_{c}, v_{c}}(N) \equiv\left[\left(1+a^{2} / 6\right)\right]^{-2},
$$

with ' $1 / 6$ ' being specific to $R^{-6}$ interaction potentials. In this regime, with increasing $a$ collisions become more and more adiabatic.

We utilized an optical centrifuge for studying collisional decoherence as a function of the degree of rotational excitation. By spinning molecules in extremely broad range of $N$ numbers, we observed more than an order-ofmagnitude change in the decoherence rates. Since in our case the control of the molecular angular momentum is executed separately from changing the gas temperature, we were able to explore the molecular dynamics at the cross-over between the non-adiabatic and adiabatic regimes of collisional relaxation, testing the ECS model beyond the adiabaticity threshold.

For this experiment, we used the Raman spectroscopy setup of section 3.1 with a narrowband probe light ( $0.1 \mathrm{~nm}$ FWHM). The gas chamber was filled with nitrogen or oxygen at room temperature, and the Raman spectrum was recorded as a function of the delay between the centrifuge and probe pulses. An example of the experimentally detected Raman spectrogram of nitrogen is shown in Fig. 6.1. The created wave packet consisted of a large number of odd and even $N$-states, corresponding to para- and ortho-nitrogen, respectively, whose 1:2 relative population ratio explains the observed alternation of amplitudes.

The intensity of each Raman line is proportional to the square of the rotational coherence $\rho_{N, N+2}$, while the frequency shift is equal to the frequency 


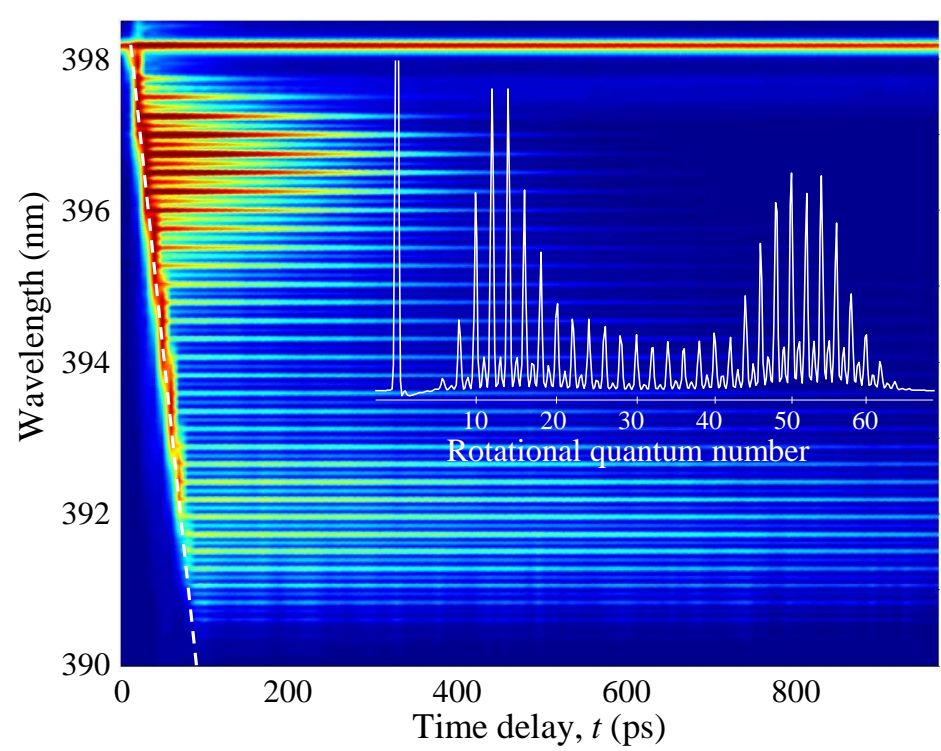

Figure 6.1: Experimentally detected Raman spectrogram of nitrogen showing the rotational Raman spectrum as a function of the time delay between the beginning of the centrifuge pulse and the arrival of the probe pulse. Color coding is used to reflect the signal strength. Tilted white dashed line marks the linearly increasing Raman shift due to the accelerated rotation of molecules inside the 100 ps long centrifuge pulse. A one-dimensional cross section corresponding to the Raman spectrum at $t=270 \mathrm{ps}$ is shown in white.

difference $\omega_{N, N+2}$ between the rotational levels separated by $|\Delta N|=2$. As one can see in Fig. 6.1, Raman lines corresponding to higher values of angular momentum decayed slower than those with lower $N$ 's. The decay was happening on the time scale of hundreds of picoseconds, much longer than the duration of our probe pulses $(\approx 4.5 \mathrm{ps})$. This offered the possibility to analyze the decay of rotational coherences with both state and time resolution. We plot the time dependence of the intensity of 50 Raman peaks on a logarithmic scale in Fig. 6.2 (colored dots). The observed decay of each Raman line is well described by a single exponential decay, in agreement with a simple theory of decoherence due to random binary collisions.

The dependence of the exponential decay rate on the rotational quantum number at room temperature is shown by blue triangles in Fig. 6.3. Expressed in the often used units of Raman linewidth $\left(\mathrm{cm}^{-1} \mathrm{~atm}^{-1}\right)$, it was 


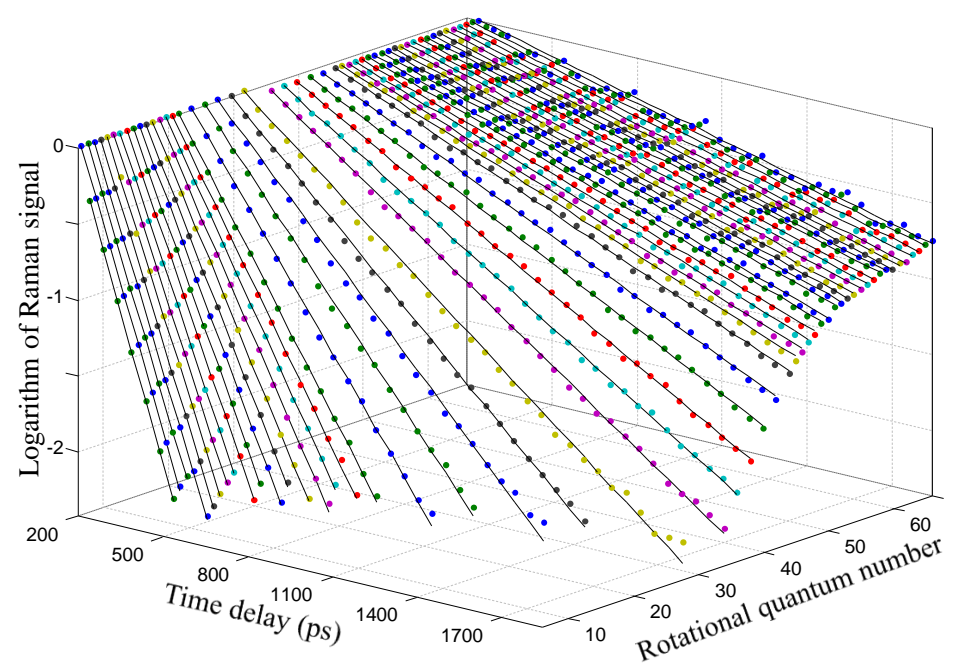

Figure 6.2: Logarithm of the intensity of the experimentally measured Raman lines as a function of time (normalized to 1 at $t=200 \mathrm{ps}$, chosen so as to avoid the effects of the detector saturation at earlier times). Dots of the same color represent experimental data for one particular value of the rotational quantum number. Black solid lines show the numerical fit to the corresponding exponential decay. Data collected at $P=0.75 \mathrm{~atm}$ and $T=294 \mathrm{~K}$.

calculated as $[67,90]$ :

$$
\Gamma_{N}=\left(2 \pi c \tau_{N}\right)^{-1}
$$

where $\tau_{N}$ is the exponential decay time of the Raman signal corresponding to the transition between states $N-2$ and $N$ (right vertical scale in Fig. 6.3, and $c$ is the speed of light in $\mathrm{cm} / \mathrm{s}$. Note that the units of $\left(\mathrm{cm}^{-1} \mathrm{~atm}^{-1}\right)$ indicate linear dependence of the decay rate on pressure, exactly as expected from a linear dependence of the collision rate on gas density. This linear relationship has been confirmed in our experiments conducted at various pressure values ranging from 0.5 to $1.5 \mathrm{~atm}$.

The observed decoherence rate dropped by more than an order of magnitude with the angular momentum of nitrogen molecules increasing from $N=8$ to $N=66$. At the lower end of the scale, $N<20$, which can be accessed at room temperature without the use of the centrifuge, our results were in good agreement with the known data from thermal ensembles [90], shown in Fig. 6.3 by black squares. At $N=10$, the decay rate corresponded 


\subsection{Effects of ultrafast rotation on collisional decoherence}

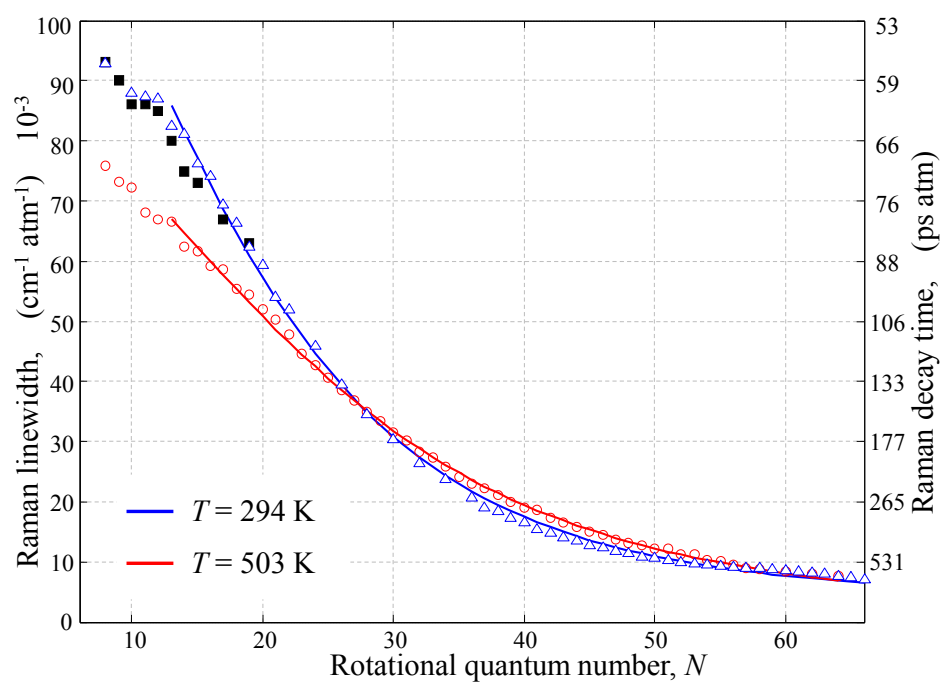

Figure 6.3: Decay rate of rotational coherence of $\mathrm{N}_{2}$ as a function of the rotational quantum number at two different temperature values, $T=294 \mathrm{~K}$ (blue triangles) and $T=503 \mathrm{~K}$ (red circles), expressed as the Raman linewidth. Solid curves correspond to the prediction of the simplified "energy corrected sudden" (ECS) approximation at $N>12$. Black squares represent the data from Ref.90.

to the exponential time constant of $62 \mathrm{ps}$, whereas it grew to 664 ps at $N=66$. Since the intensity of Raman signal scales as the square of the rotational coherence, this yielded the coherence lifetime of $1.33 \mathrm{~ns}$, or the equivalent of about 10 collisions. In contrast to "slow rotors" whose dynamics are altered by a single collision, super rotors are much more resilient to collisional relaxation.

We start the analysis of the observed behavior by first noting that our experimental technique cannot distinguish between the elastic and inelastic mechanisms of rotational decoherence. The latter mechanism, however, has been suggested as the main contributor to the decay of rotational coherence [42, 121]. It is therefore instructive to examine the utility of the energy corrected sudden approximation, which gives the recipe for calculating the rate of inelastic $N$-changing collisions, in describing our experimental findings. In ECS, the rate of transition from $N$ to $N^{\prime}$ is given by the follow- 
ing expression:

$$
\begin{gathered}
\gamma_{N, N^{\prime}}^{\mathrm{ESC}}=\left(2 N^{\prime}+1\right) \exp \left(\frac{E_{N}-E_{N_{>}}}{k_{B} T}\right) \times \\
\sum_{L}\left(\begin{array}{ccc}
N & N^{\prime} & L \\
0 & 0 & 0
\end{array}\right)^{2}(2 L+1) \frac{\Omega_{l_{c}, v_{c}}(N)}{\Omega_{l_{c}, v_{c}}(L)} \gamma_{L 0},
\end{gathered}
$$

where $E_{N}$ is the rotational energy (with $N_{>}$denoting the largest value between $N$ and $\left.N^{\prime}\right),(:::)$ is the Wigner $3 J$ symbol, and $\Omega_{l_{c}, v_{c}}(N)$ is the correction factor introduced earlier. Given that the basic rate $\gamma_{L 0}$ is known to fall off rather steeply with $L[12$, we simplify Eq. 6.3 by leaving only the single dominant term with $L=2$ in the sum. Because of the exponential gap factor in the first line, we also assume that the main contribution to the decay of rotational coherence comes from the downward transitions with $N^{\prime}=N-2$. Noticing that the corresponding $3 J$ symbol scales as $1 / \sqrt{N}$ at large $N$, we arrive at the following simplified decay rate:

$$
\Gamma_{N \gg 1} \approx \underset{\substack{N, N-2 \\(N \gg 1)}}{\gamma_{N S C}} \approx \frac{15 \gamma_{20}}{8 \Omega_{l_{c}, v_{c}}(2)} \Omega_{l_{c}, v_{c}}(N) \equiv \Gamma_{0} \Omega_{l_{c}, v_{c}}(N)
$$

where all the factors which are independent on $N$ have been included in $\Gamma_{0}$. With only two fitting parameters, $\Gamma_{0}$ and $l_{c}$, this simple expression results in a reasonably good agreement with our experimental observations in the region of high $N$ values. This is demonstrated by fitting the data using Eq. 6.4 at $N>12$, i.e. above the visible bend in the curve predicted by the full ECS model and observed here similarly to a number of previous reports [67, 89, 90]. The fit (solid blue line in Fig. 6.3) corresponds to the characteristic interaction length $l_{c}=0.74 \pm 0.03 \AA$, in excellent agreement with the previously reported value of $0.75 \AA[89,123]$.

To test the proposed simplified scaling law, we repeated the experiment at a higher temperature. A small heater was placed inside the chamber to heat the gas up locally to $T=503 \mathrm{~K}$. The measured decay rates are shown with red circles in Fig. 6.3. Importantly, fitting the high-temperature data for $N>12$ with Eq. 6.4 yields the same characteristic length $l_{c}=0.74 \AA$, confirming the validity of the model. In accord with all previous observations of thermally accessible $N$ levels, increasing the temperature results in a slower decay rate, primarily due to the reduced gas density. Increasing the value of $N$ leads to the similar decrease of $\Gamma_{N}$, this time due to the growing adiabaticity of collisions. However, as can be seen from Fig. 6.4, the colder the ensemble the faster the growth of the adiabaticity factor $a$ with $N$. This 


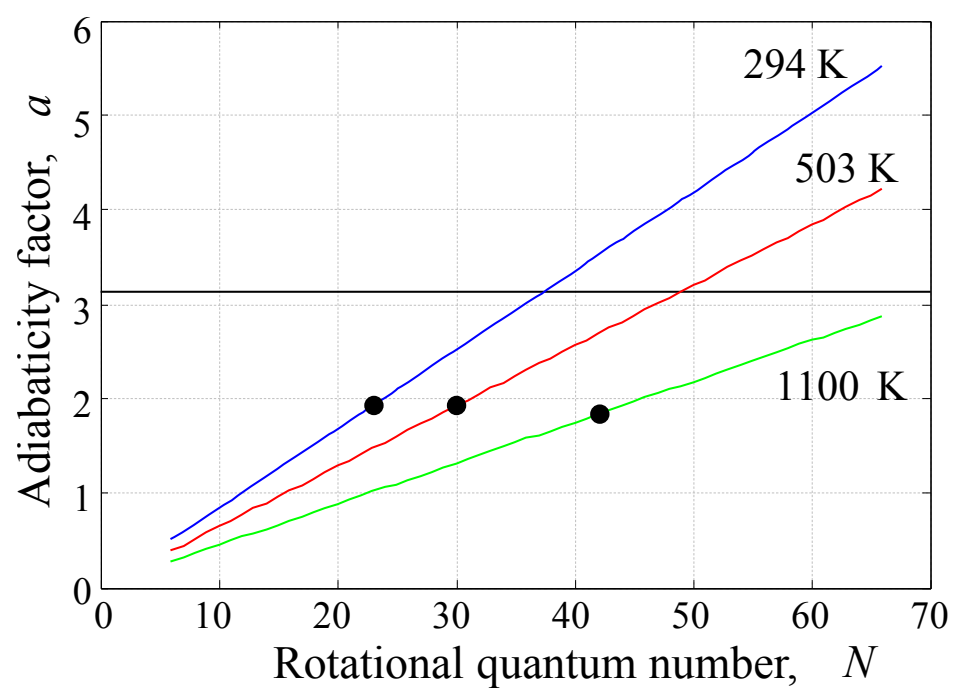

Figure 6.4: The dependence of the adiabaticity factor $a$ on $N$ for the nitrogen gas temperatures of $294 \mathrm{~K}$ (blue line), $503 \mathrm{~K}$ (red line) and $1100 \mathrm{~K}$ (green line). Solid horizontal line marks the adiabaticity threshold, $a=\pi$. Black filled circles mark the rotational evels accessed in previous experiments on rotational decoherence.

fact explains the observed reversal of the temperature dependence between $N \approx 35$ and $N \approx 50$, where the molecules in a hot (and therefore more dilute) ensemble lose their rotational coherence faster than the denser cold ones. In this window of $N$ 's, the value of $a$ is already above the adiabaticity threshold at $T=294 \mathrm{~K}$, yet still below it at $T=503 \mathrm{~K}$.

Black circles in Fig. 6.4 mark the highest observed states of nitrogen at $T=300,500$ and $1100 \mathrm{~K}$ (also corresponding to the rotational levels containing $0.2 \%$ of the total rotational population, which seems to represent the smallest fraction of molecules, detectable in a typical Raman experiment). One can see that, although the range of $N$ values can be extended by increasing the temperature, the maximum accessible adiabaticity parameter remains constant and rather low. Controlling molecular rotation with an optical centrifuge eliminated this limitation and enabled us to cross over to and explore the adiabatic regime of rotational decoherence.

Similar measurements were carried out in oxygen. Since the observed time dependencies was complicated by the spin-rotational dynamics section 4.3), the fitting procedure to extract the time constant $\tau_{N}$ of the 


\subsection{Effects of ultrafast rotation on collisional decoherence}

collision-induced exponential decay was modified to account for the SR oscillations. Similar to nitrogen, for the slower rotating molecules, the coherence life time is shorter than for the faster rotors. This is shown with blue circles in Fig. 6.5, where the decay rate is plotted as a function of the angular frequency of molecular rotation. Black asterisks and grey squares depict the previously reported data obtained in a thermal ensemble of oxygen molecules at room temperature (hence, $N \leqslant 25$ ) using frequency-resolved $[92]$ and timeresolved [90] detection techniques, respectively. Our results at low $N$ 's show satisfactory agreement with both data sets.

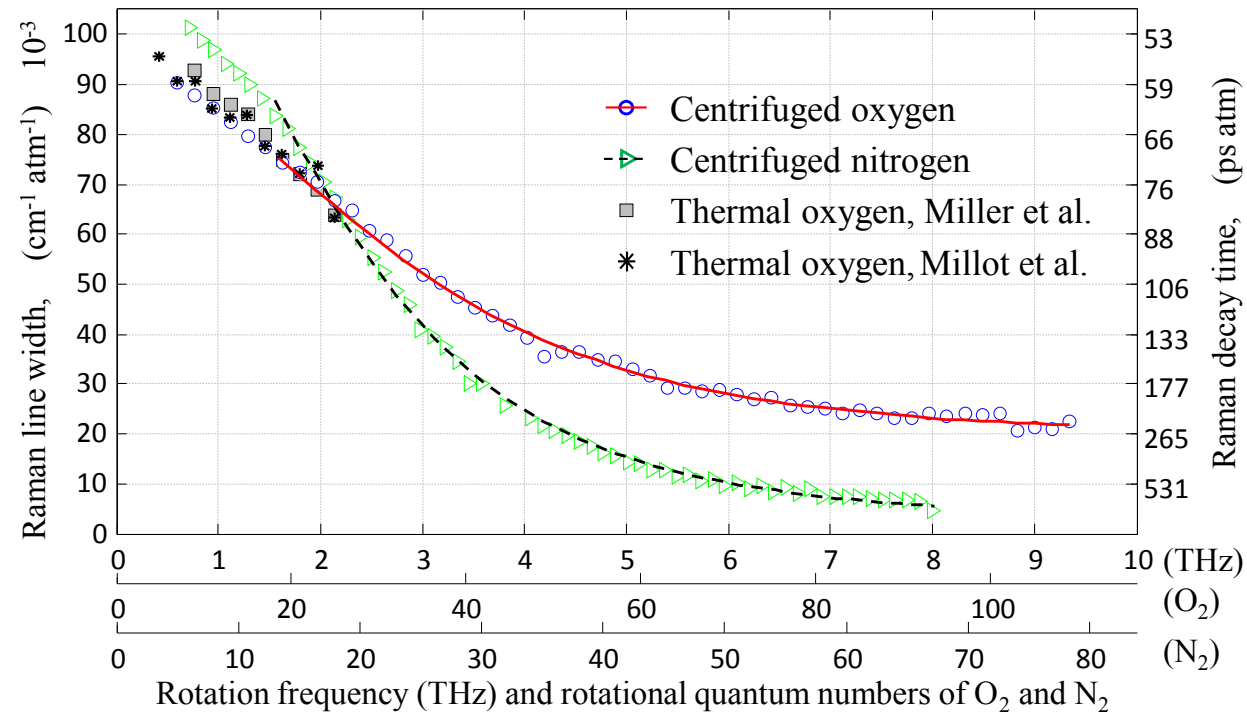

Figure 6.5: The decay rate of rotational coherence in oxygen (blue circles) and nitrogen (green triangles) as a function of the frequency of molecular rotation. For convenience, rotational quantum numbers of $\mathrm{O}_{2}$ and $\mathrm{N}_{2}$ are shown below the frequency axis, and the decay times $\tau_{N}$ are shown on the right vertical axis. Black asterisks and grey squares depict the data from 92 and 90], respectively, where the rotational decay has been studied in thermal oxygen by two different techniques. Solid red (dashed black) curve shows the result of fitting the adiabaticity correction factor $\Omega_{l_{c}}(N)$ to the data for oxygen (nitrogen) as discussed in text.

We repeated the fitting procedure as before, to extract $\Gamma_{0}$ and $l_{c}$ parameters of the ECS model. Unlike nitrogen, an additional fitting parameter 
$\Gamma_{\infty}$ was required to achieve a good agreement with experiment:

$$
\Gamma_{N}=\Gamma_{0} \Omega_{l_{c}}(N)+\Gamma_{\infty}
$$

Red solid curve in Fig. 6.5 shows the result of fitting Eq.6.5 to our data in the range of high adiabaticity $\left(a_{N}>1\right)$. Good fit was achieved with the values $\Gamma_{0}=76.9 \times 10^{-3} \mathrm{~cm}^{-1} \mathrm{~atm}^{-1}, \Gamma_{\infty}=19.6 \times 10^{-3} \mathrm{~cm}^{-1} \mathrm{~atm}^{-1}$ and $l_{c}=0.56$ $\AA$. The latter is close to the value found from applying the ECS model in the limit of low angular momentum[123], which may suggest a possibility of extending the known scaling law to the limit of high adiabaticity using the same correction factor.

The necessity of adding an additional parameter $\Gamma_{\infty}$ for oxygen may point at a non-negligible contribution of the spin-flipping collisions in the decay of coherent Raman scattering in the gas of magnetic $\mathrm{O}_{2}$ molecules. The cross section for the spin relaxation of oxygen at room temperature is not known and its exact calculation is a formidable task. However, an insight can be gained from the recent studies of this process at low temperatures 4,125 . As the temperature exceeds $\approx 0.01 \mathrm{~K}$ and approaches $1 \mathrm{~K}$, the ratio between the elastic and spin-flipping collisions has been found to reach a constant of order 10, which shows little dependence on $T$. When the temperature rises above $1 \mathrm{~K}$, the energy scale of the spin-rotation splitting becomes less significant and no dramatic changes in the elastic-to-inelastic cross section ratio are expected. Given this argument, the spin-flipping cross section may indeed be somewhat below, yet comparable to, the cross section of the rotational relaxation at low $N$ values.

Alternatively, faster decoherence of oxygen rotation may stem from the higher concentration of $\mathrm{O}_{2}$ super rotors with respect to the concentration of the centrifuged $\mathrm{N}_{2}$. This empirically found difference may result in the increasing local gas temperature and correspondingly higher rates of rotational energy transfer.

\subsection{Crossover from gyroscopic to thermal motion}

As the super rotors underwent collisional relaxation, their rotational energy was released, driving the temperature of the gas up. The rotationtranslation energy transfer was accompanied by a crossover from the nonequilibrium gyroscopic to the thermal phase of molecular dynamics. Hydrodynamics of a locally heated gas result in the corresponding change in its pressure, followed by the emission of an acoustic wave and the formation of 
a low-density thermal channel. In this section, we describe our experimental and numerical investigation of this process.

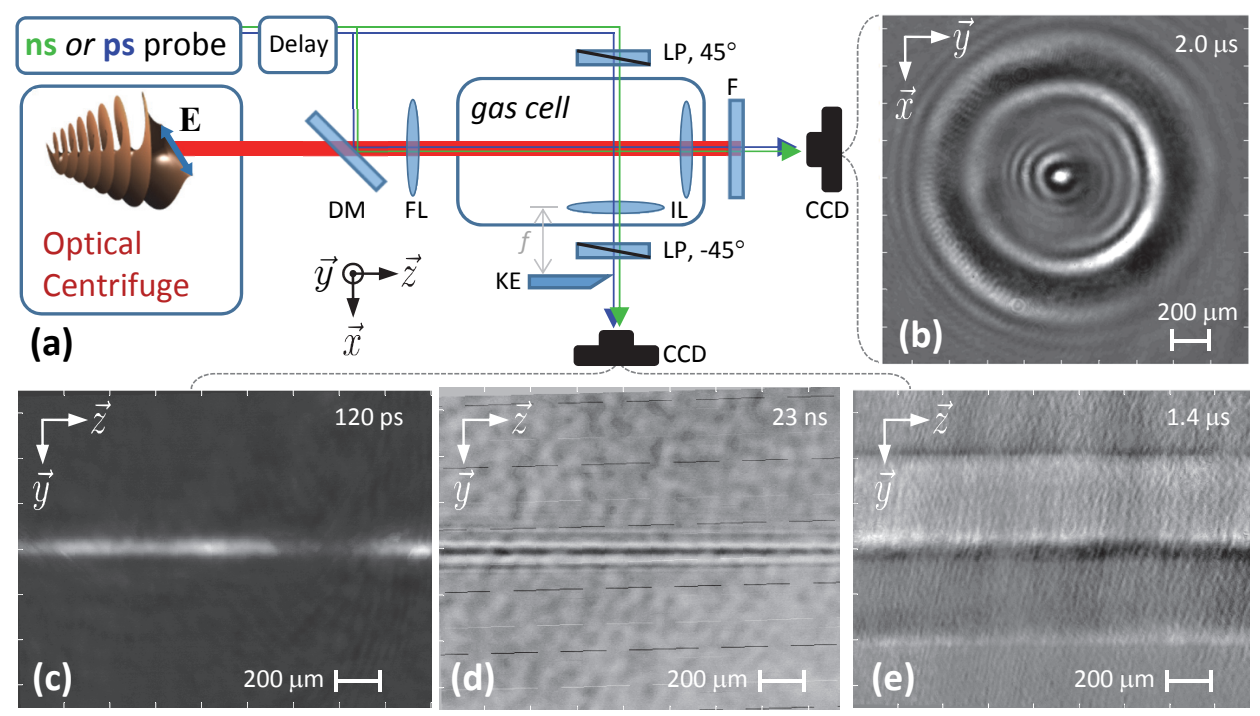

Figure 6.6: (a) Schematic diagram of the imaging setup. Nanosecond or picosecond probe pulses (green and blue beams, respectively) propagated either collinear with, or perpendicular to the centrifuge, creating an image of the rotationally excited volume of gas on a CCD camera either in the longitudinal or transverse direction, respectively. DM: dichroic mirror, FL: focusing lens, IL: imaging lens, KE: knife edge (shown along $y$ instead of $z$ axis for clarity), LP: linear polarizers at $\pm 45^{\circ}$ to $y$ axis, F: frequency filter. An example of the longitudinal image with a circularly expanding sound wave is shown in panel (b). Images in the transverse geometry were taken at early (c), intermediate (d) and late (e) time moments. Image (c) was recorded with the two linear crossed polarizers in place. Schlieren image (e) was recorded with the knife edge in place.

Our experimental configuration is depicted in Fig. 6.6. The centrifuge beam was focused by a weak $1 \mathrm{~m}$ focal length lens down to a spot size of $90 \mu \mathrm{m}$ diameter (full width at half maximum) inside the Raman chamber filled with 0.9 bar of oxygen. The performance of the centrifuge was monitored by means of coherent Raman spectroscopy, described earlier.

We imaged the volume of the centrifuged gas both on short and long time scales, $\tau<23 \mathrm{~ns}$ and $\tau<2 \mu \mathrm{s}$, respectively. For a short-time scan, 
picosecond pulses were extracted from the same Ti:Sapphire ultrafast system as the centrifuge, and delayed with femtosecond precision by means of computer controlled motorized translation stages. Long-time scans were executed with nanosecond pulses from a separate YAG laser, while their delay was controlled electronically. In both cases, single-shot images were recorded with a CCD camera and typically averaged over 400 laser pulses.

Two imaging geometries were implemented: longitudinal and transverse, with probe pulses propagating collinear with, or at 90 degrees to the direction of the centrifuge. A sample longitudinal image, taken $2 \mu$ s after the centrifuge pulse, is shown in the upper right corner of Fig. 6.6. The location of the central bright spot, surrounded by multiple circular interference fringes, corresponds to the position of the centrifuge beam. The fringe pattern stems from the diffraction of probe pulses inside the centimeter-long density depression channel. Owing to the linearity of the weak probe propagation and its extended length, the fringe contrast serves as a sensitive indicator of the centrifuge-induced changes in the refractive index of the gas, $\Delta n$.

We analyzed the time dependence of $\Delta n$ by recording a series of longitudinal images while scanning the delay time between the centrifuge and probe pulses. An angle-averaged radial cross-section of the interference pattern, recorded with the nanosecond laser as a probe, was calculated and plotted as a function of the delay in Fig. 6.7. The plot shows a permanent central core and an outgoing sound wave, similar to the recently observed dynamics of plasma filaments[75, 133. Unlike the case of plasma, however, zooming in on the first few nanoseconds of the filament formation reveals unique details of its early history, shown in Fig. 6.8. The instantaneous creation of a refractive channel by the centrifuge, reflects its non-thermal origin. Indeed, we detected the signal already in the first picoseconds after the arrival of a centrifuge pulse - too short a time scale to allow collisional thermalization. Instead, the collisions seem to suppress, rather than enhance $\Delta n$, which disappears almost completely at around 7 ns. From that moment on, the channel grows back and eventually emits the sound wave seen in Fig. 6.7, in accord with the expected thermal dynamics. A clear crossover between the two regions is illustrated in panel (b) of Fig. 6.8, where we plot the contrast of the recorded longitudinal images as a function of time. The universality of the crossover is demonstrated in Fig. 6.9, where it is observed in the gas of $\mathrm{N}_{2} \mathrm{O}$ super rotors at two different pressures. Collision-driven dynamics are evident from the increasing lifetime of the gyroscopic stage with decreasing pressure. To identify the nature of the refractive channels before and after the crossover, we switched to the transverse imaging geometry. 


\subsection{Crossover from gyroscopic to thermal motion}

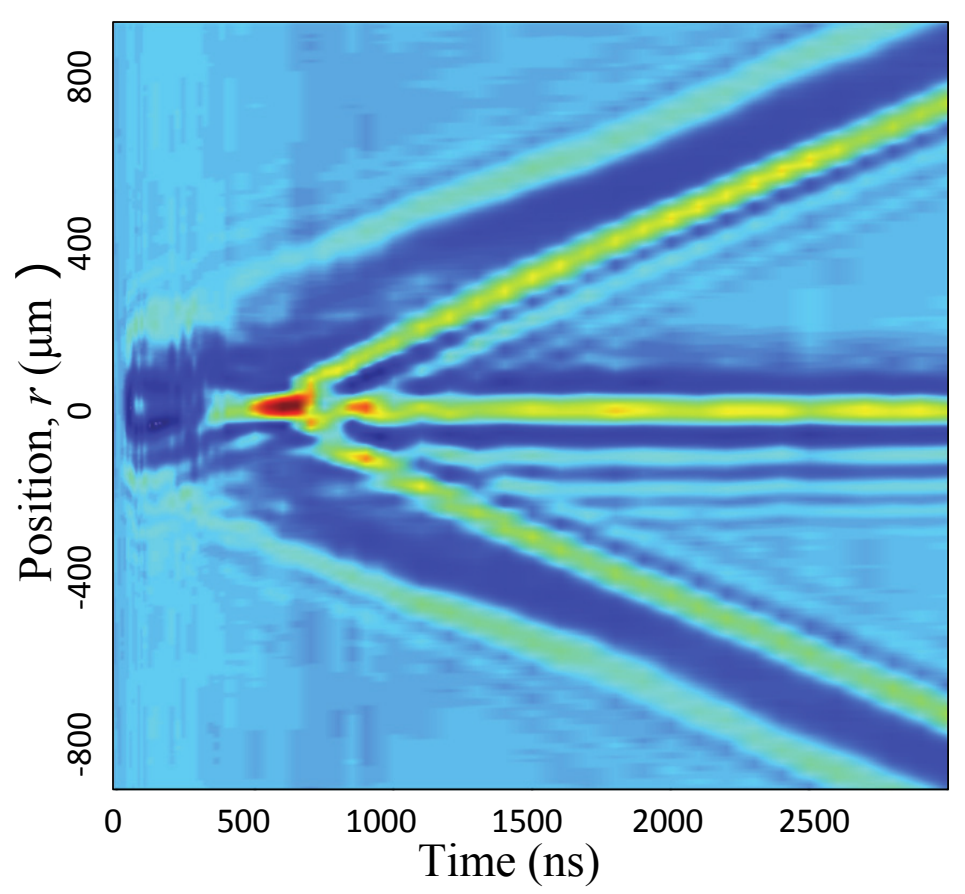

Figure 6.7: Radial cross-section of the images, representing the change in the refractive index of the gas, taken along the centrifuge beam, as a function of time, recorded on a long timescale.

Three typical transverse images, recorded on three qualitatively different time scales, are presented at the bottom of Fig. 6.6. The schlieren technique 114] was employed by inserting a knife edge in the focal plane of the imaging lens, to determine the true distribution of the refractive index (more exactly, its $\mathrm{d} / \mathrm{d} y$ derivative) at long delay times. The side view of a narrow channel is free of interference fringes, seen in the longitudinal geometry, which greatly simplifies the interpretation of the picture. Schlieren image (e) on the right shows two high-density waves captured $400 \mu \mathrm{m}$ above and below the low-density depression channel in the center. The snapshot (d) in the middle was taken close to the very origin of the thermal channel around $\tau \approx 20 \mathrm{~ns}$, before the beginning of the hydrodynamic expansion of the gas. At even shorter delay times of order of, and below, 5 nanoseconds, our transverse phase-contrast imaging approached its signal-to-noise limit. To further explore the molecular dynamics at these early moments, we probed the centrifuge-induced birefringence of the gas by taking the images 


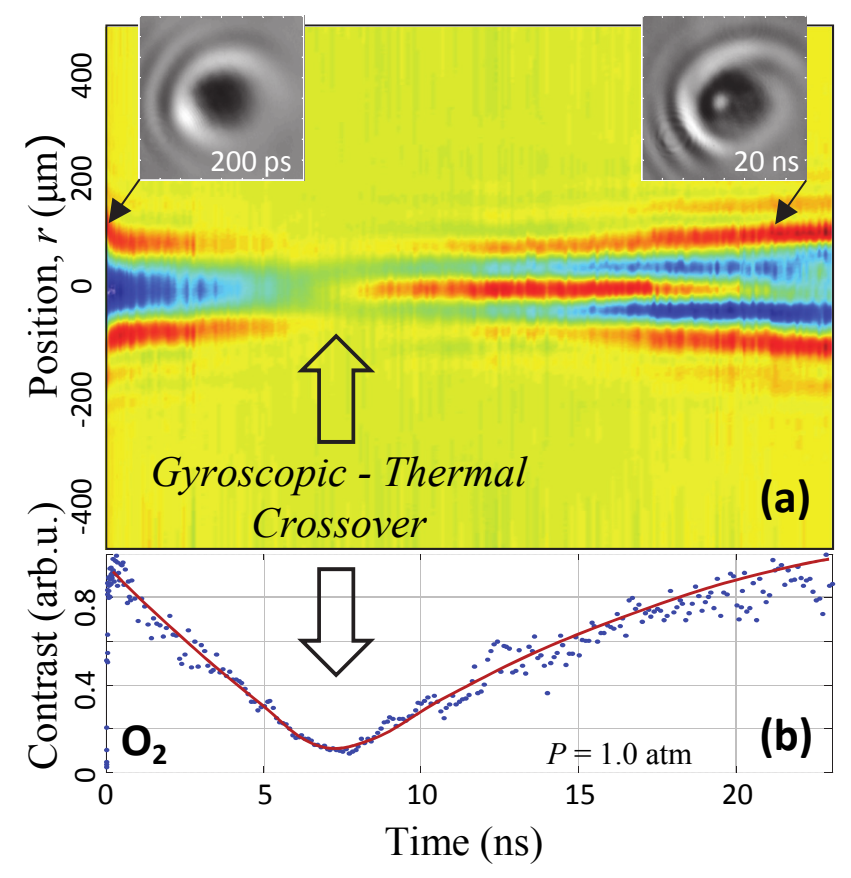

Figure 6.8: (a) Radial cross-section of the images, taken along the centrifuge beam, as a function of time, recorded on a short timescale. The arrow at around 7 ns shows the crossover between the rotation-induced and thermal channels. Sample images of both refractive channels are shown in the square insets. (b) Image contrast as a function of time. The red line on top of the experimental data points is shown to guide the eye.

between two crossed linear polarizers (leftmost picture (c) at the bottom of Fig. 6.6).

The difference between the two processes affecting optical properties of the centrifuged gas before and after the observed crossover became apparent when we compared the time dependence of the detected optical birefringence with that of the image contrast. The two observables, extracted from the images taken with and without the crossed polarizers, respectively, are plotted in Fig. 6.10. The birefringence signal is expressed in arbitrary units with 1 being equivalent to the polarization rotation angle of about $1 \mathrm{mrad}$. This birefringence, instantaneously induced by the centrifuge, falls off exponentially with a time constant of $3.4 \mathrm{~ns}$ (solid black curve). From these results, the disappearing channel to the left of the crossover in Fig. 6.8 can 


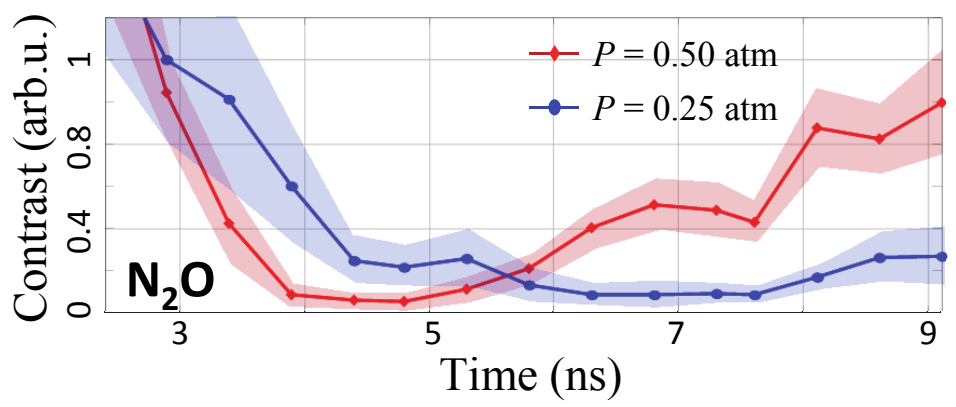

Figure 6.9: Radial cross-section of the images, taken along the centrifuge beam, as a function of time recorded in the gas of $\mathrm{N}_{2} \mathrm{O}$ molecules at the pressure of $0.5 \mathrm{~atm}$ (red line) and $0.25 \mathrm{~atm}$ (blue line). Shaded regions around each line represent the statistical error (one standard deviation) in our experimental data.

be clearly correlated with the decaying birefringence signal, and therefore associated with an optical anisotropy induced by the directional molecular rotation. As the molecular super rotors lose their rotational energy to heat, this gyroscopic channel dies off while thermal effects take over, causing an isotropic change in the gas density reflected in the growing image contrast at $\tau \gtrsim 7$ ns.

Note that the longitudinal imaging is more sensitive to the small changes of the refractive index at early times, when it is changing monotonically across the refractive channel, and not as sensitive to its further growth later on, when the developing fine structure (due to the formation of an acoustic wave) results in multiple diffraction fringes. This explains the fact that the longitudinal image contrast before and after the crossover in Fig. 6.8 (b) is of the similar magnitude, unlike the case of the transverse contrast in Fig. 6.10, which disappears below the cross-over time. To make sure that super rotors are responsible for the observed optical changes, we repeated the experiments with the centrifuge pulses of the same peak intensity but lower terminal rotation frequency. Two points inside the dashed rectangle in Fig. 6.10, upper square and lower diamond, correspond to the centrifuge producing only slow rotors and no rotors at all, respectively.

To verify the mechanism behind the rotation-translation crossover, we numerically simulated the hydrodynamics of the ideal gas exposed to a 


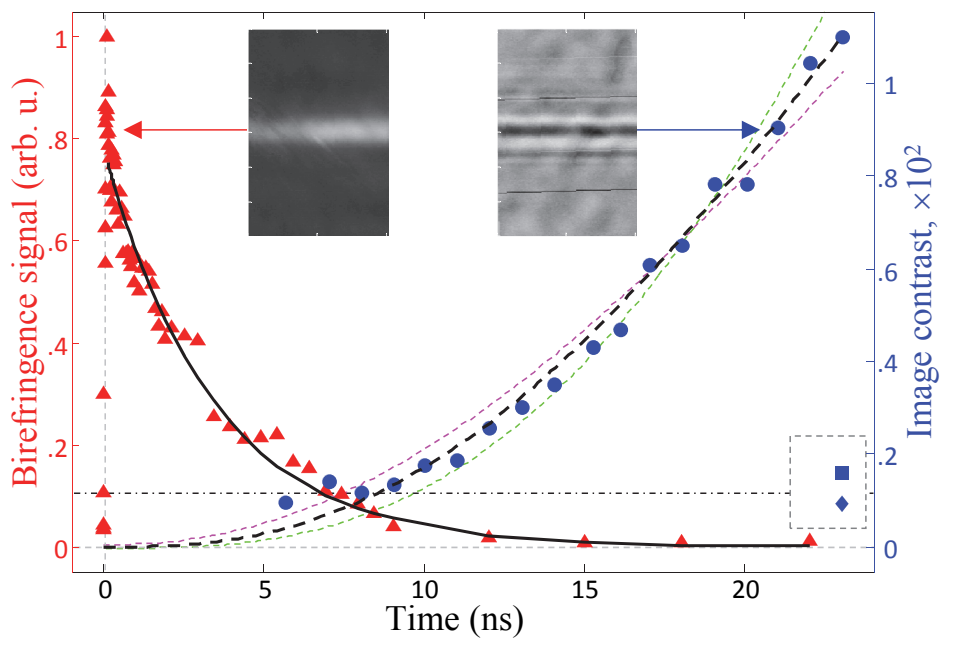

Figure 6.10: Birefringence signal (red triangles) and image contrast (blue circles) of the centrifuged gas, retrieved from the transverse images taken with and without the crossed polarizers (left and right insets, respectively) as a function of time. Two data points in the lower right corner (within the dashed rectangle) indicate the drop in the phase contrast for the slower rotating centrifuge. The solid black line is a fit to an exponential decay of the birefringence signal, which gave $\tau_{b}=3.4 \mathrm{~ns}$ as the decay time. This time constant was used in the hydrodynamic numerical calculations of $\Delta n(\tau)$, shown by the dashed black line. The dashed magenta and green lines correspond to the same calculations, performed with the decay constant being five times shorter and longer, respectively. The horizontal dash-dotted line indicates the noise floor for the phase contrast measurement.

known heat source, using the equations:

$$
\begin{aligned}
\partial / \partial t(\rho) & =-\nabla[\rho \mathbf{v}] \\
\partial / \partial t(\mathbf{p}) & =-\nabla[\mathbf{p} \mathbf{v}+P], \\
\partial / \partial t(\mathcal{E}) & =-\nabla[\mathcal{E} \mathbf{v}+P \mathbf{v}+\mathbf{q}],
\end{aligned}
$$

where $\rho, \mathbf{p}=\rho \mathbf{v}$ and $\mathcal{E}=\frac{1}{2} \rho v^{2}+\varepsilon$ are the mass, momentum and energy of the unit gas volume; $\mathbf{v}$ its velocity and $\varepsilon=\rho c_{\mathrm{v}} T$ its internal energy, with $c_{\mathrm{V}}$ being the heat capacity at constant volume and $\mathrm{T}$ the gas temperature. $P$ is the pressure of the gas and $\mathbf{q}=-\kappa \nabla T$ is the heat flux, with $\kappa$ being the heat conductivity. These equations, corresponding to the conservation 
of mass, momentum and energy in the compressible gas flow, were solved for $\rho, \mathbf{v}$ and $P$, assuming cylindrical symmetry and equilibrium initial and boundary conditions. Rotation-translation energy exchange was modeled by adding an external pressure source, $\frac{1}{P_{0}}(\partial P / \partial t)_{\mathrm{RT}}=\frac{\Delta T}{T_{0}} \frac{1}{\tau_{b}} e^{-t / \tau_{b}}$, to the corresponding differential equation for $P$, where $T_{0}\left(P_{0}\right)$ is the ambient temperature (pressure) and $\Delta T$ is the temperature increase at the end of the thermalization process.

An exponential increase of the gas temperature (and hence, its pressure in the first few nanoseconds) with the same time constant $\tau_{b}=3.4 \mathrm{~ns}$ as the decay of the birefringence signal, stems from the proportionality of both the birefringence signal and the rotational energy to $J^{2}$. Indeed, the birefringence signal is proportional to $\left(1-3\left\langle\cos ^{2} \theta_{z}\right\rangle\right)^{2}$ [49], becoming non-zero when the molecules are confined to the $x y$ plane $\left(\left\langle\cos ^{2} \theta_{z}\right\rangle<1 / 3\right)$. For a gas of molecules in the quantum state $\left|J, M_{J}=J\right\rangle$, one finds: $\left\langle\cos ^{2} \theta_{z}\right\rangle=$ $\left\langle J, J\left|\cos ^{2} \theta_{z}\right| J, J\right\rangle=(2 J+3)^{-1}$. As a result, the birefringence signal is proportional to $J^{2}$, as well as the rotational energy, and should therefore be described by the same exponential $\tau_{b}$.

To estimate $\Delta T$, we note that the peak intensity of our centrifuge pulses is sufficient to spin adiabatically only $2 \%$ of $\mathrm{O}_{2}$ molecules occupying the lowest rotational state at room temperature. After these molecules are excited to $J \approx 91$ by the centrifuge (as determined by means of Raman spectroscopy), each oxygen super rotor carries $1.5 \mathrm{eV}$ of rotational energy. Redistributing this energy among the whole molecular ensemble and all 5 degrees of freedom results in $\Delta T=140 \mathrm{~K}$.

The main result of our hydrodynamic calculations is shown in Fig.6.11(a), where the change in the gas density, $\Delta \rho(y, \tau)$, is plotted as a function of both the distance from the centrifuge and the time since its arrival. One can see the density depression channel forming in the center in the first $200 \mathrm{~ns}$ and an acoustic density wave spreading radially away from it. Given the linear dependence of the refractive index of a gas on its density, we compare the experimentally observed image contrast with the numerically calculated $|\Delta \rho(y=0, \tau)|$ in Fig. 6.10 (blue circles and dashed black line, respectively). The only fitting parameter was the proportionality coefficient between the gas density and the image contrast. An excellent agreement between the experimental and numerical line shapes supports our interpretation of the gyroscopic channel and its role as the energy source for the thermal gas expansion. If the RT exchange rate was assumed to be very different from the birefringence decay rate $\tau_{b}$, the numerical results did not fit the experimental data as well (dashed magenta and green lines in Fig. 6.10).

Further comparison between our experimental findings and numerical 


\subsection{Crossover from gyroscopic to thermal motion}

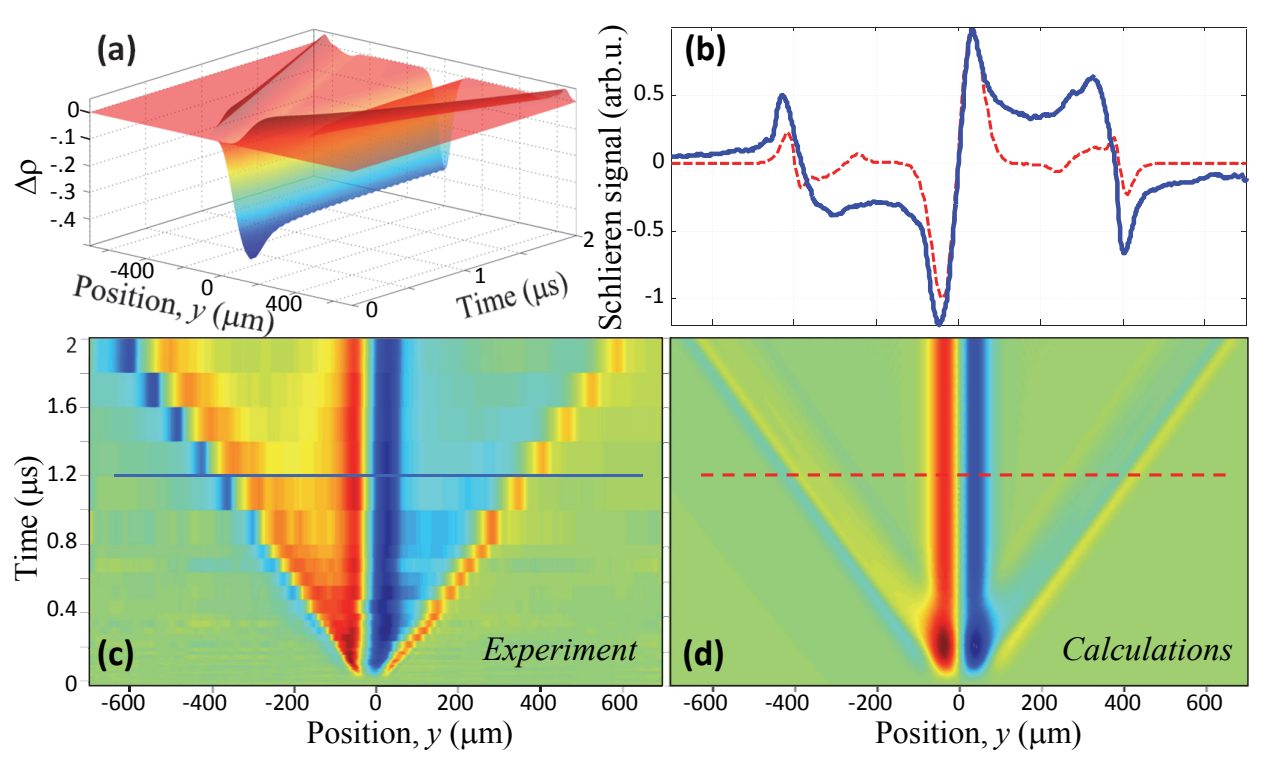

Figure 6.11: Comparison of the experimental results with the numerical calculations of gas hydrodynamics. The calculated change in the gas density, $\Delta \rho$, is shown in panel (a) as a function of time and distance. Experimentally determined parameters of the observed gyroscopic channel are used to simulate the heat source, which initiates the dynamics at point $(y=0, \tau=0)$. In panel (b), the derivative of the calculated density profile $\mathrm{d} / \mathrm{d} y[\rho(y)]$ at $1.2 \mu \mathrm{s}$ is compared with the $y$ cross-section of the schlieren image recorded $1.2 \mu$ s after the centrifuge (dashed red and solid blue curves, respectively). The dependence of the measured and calculated schlieren signals on both space and time is shown in panels (c) and (d), respectively, with the two lines indicating the two cross-sections displayed in plot (b).

model is presented in Fig. 6.11 (b). The schlieren signal at $1.2 \mu$ s is calculated by taking the derivative of $\Delta \rho(y, \tau=1.2 \mu \mathrm{s})$ with respect to $y$, and plotted together with the $y$ cross-section of the experimental schlieren image taken at that delay time (cf. Fig. 6.6 (e)). The dependence of schlieren signals on both $y$ and $\tau$ is shown in Fig. 6.11 (c) and (d) for the experimental and numerical data, respectively. The experimental scan, carried out with ns probe pulses, extends to $2 \mu$ s and shows an acoustic wave traveling with the speed of sound away from the central core. The equivalent numerical scan displays very similar behavior, supporting the validity of the model. 


\subsection{Long-lived permanent molecular alignment}

As was discussed in section 5.1, a 2D centrifuge can be used to align diatomic molecules in extreme rotational states. Reaching high $N$ states has an immediate advantage over the conventional non-adiabatic alignment with a linearly polarized femtosecond pulse (referred to as a 1D kick). Indeed, as has been demonstrated above, the decay of both the rotational coherence (section 6.1) and the rotational energy section 6.2) of molecular super rotors due to collisions is much slower than that of slowly rotating molecules. Here, we compare the decay of molecular alignment, induced by a 1D kick and a $2 \mathrm{D}$ centrifuge in oxygen under ambient conditions, showing significantly longer decay times in the latter case.

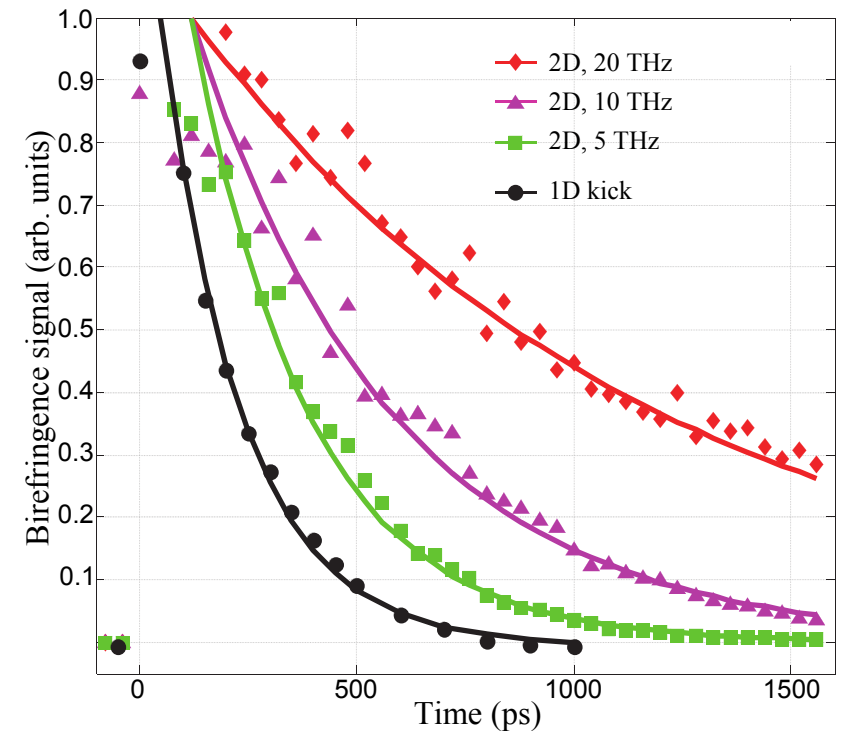

Figure 6.12: Comparison of the birefringence decay for the 2D centrifuge with a full spectral bandwidth of $20 \mathrm{THz}$ (red diamonds) and with its bandwidth truncated at $\approx 10 \mathrm{THz}$ (purple triangles) and $\lesssim 5 \mathrm{THz}$ (green squares). Black circles represent the case of a 1D kick. In all plots, solid lines show the fits by exponential decays.

We analyzed this decay of molecular alignment by repeating the birefringence measurements of section 6.2 with a $1 \mathrm{D}$ kick and a $2 \mathrm{D}$ centrifuge. In this study we used the transverse geometry, i.e. sent our probe pulses perpendicularly to the plane of molecular rotation ( $x z$ plane in Fig.5.1). Ap- 
plying the $2 \mathrm{D}$ centrifuge resulted in the nonzero linear birefringence (red diamonds in Fig. 6.12), stemming from the rod-shaped molecular distribution. Similarly to the decoherence rate of molecular super rotors (section 6.1), the decay of the permanent molecular alignment becomes slower for faster rotating molecules due to the increased adiabaticity of collisions 62$]$. We demonstrated this effect by comparing the decay rates of the birefringence signal observed with the $2 \mathrm{D}$ centrifuges which had different spectral bandwidths and, therefore, different terminal rotational frequencies. As shown in Fig 6.12 , increasing the centrifuge bandwidth from $\lesssim 5 \mathrm{THz}$ (green squares) to $\approx 8 \mathrm{THz}$ (purple triangles) and $20 \mathrm{THz}$ (red diamonds), resulted in the respective increase of the exponential decay time from $269 \pm 9$ ps to $461 \pm 15$ ps and $1094 \pm 142$ ps.

Also plotted in Fig.6.12 is the decay of the permanent molecular alignment induced by a single femtosecond (1D) kick (black circles). One can clearly see the noticeably shorter life time of this alignment $(187 \pm 8 \mathrm{ps})$ with respect to that offered by a $2 \mathrm{D}$ centrifuge. Similarly to the previously discussed increasing decay rates with a slower centrifuge, the effect stems from the lower adiabaticity of collisions between the molecules exposed to a $1 \mathrm{D}$ kick in comparison to those excited by a $2 \mathrm{D}$ centrifuge.

\subsection{Sound emission from the gas of molecular super rotors}

We utilized the tunability of the centrifuge to vary the amount of rotational energy deposited into the gas of $\mathrm{N}_{2}$ or $\mathrm{O}_{2}$ molecules while recording the acoustic signal produced by the super rotors. The latter was clearly audible to the unaided ear due to the ultra-high rotational energies exceeding $2.5 \mathrm{eV}$ per oxygen super rotor. The acoustic signal was detected by a microphone positioned perpendicularly to the centrifuge beam $6 \mathrm{~cm}$ away from its focal spot. The sensitivity of the microphone was $-31 \mathrm{dBV}(\approx 28 \mathrm{mV} / \mathrm{Pa})$ at $1 \mathrm{KHz}$ under ambient conditions, and its output was connected directly to the computer's analog-to-digital converter with no further amplification. An example of the recorded acoustic signal, averaged over 100 laser pulses, is shown in the inset to Fig. 6.13. Frequency bandwidth of the microphone was not sufficiently broad for resolving the effectively instantaneous pressure burst, which resulted in the decaying oscillatory waveform. Hereafter, we

refer to the maximum value of this waveform as the amplitude of the acoustic signal $S$.

The acoustic response of various gases to the field of an optical centrifuge 


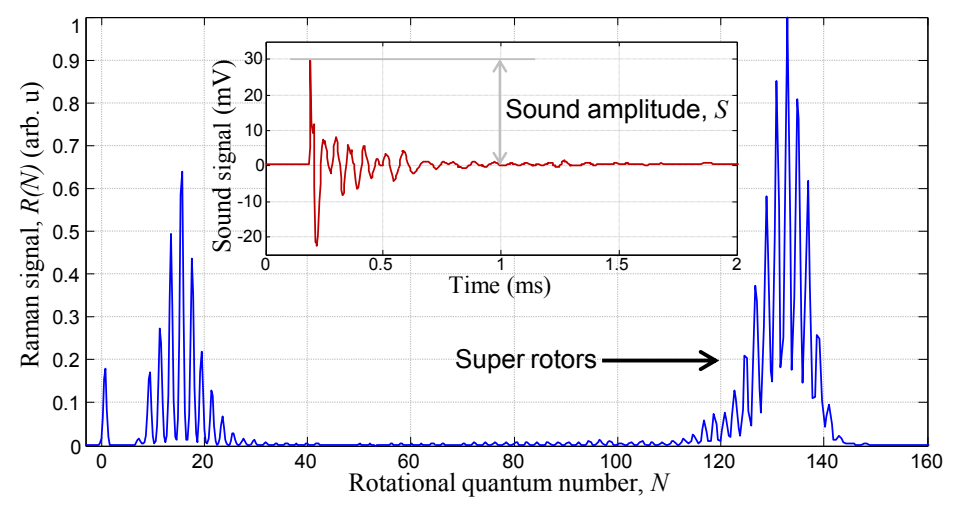

Figure 6.13: Typical rotational Raman spectrum of oxygen super rotors measured with a probe pulse delayed by $200 \mathrm{ps}$ with respect to the centrifuge pulse. Inset: an example of the acoustic signal recorded in centrifuged ambient air.

is shown in Fig. 6.14 as a function of the laser pulse energy. We first notice the difference between the sound amplitude detected in the ensemble of nitrogen super rotors and in pure argon gas under the same pressure and temperature, clear from Fig. 6.14 (a). Even though the ionization energies of the two gases are almost the same, $15.58 \mathrm{eV}$ for $\mathrm{N}_{2}$ and $15.76 \mathrm{eV}$ for $\operatorname{Ar}$ [51, the acoustic signal in nitrogen is an order of magnitude stronger. This points at the non-ionizing nature of the sound waves recorded in the gas of super rotors.

To examine this hypothesis further, we intentionally introduced ionization by adding a small amount of $\mathrm{SF}_{6}$ molecules into the gas of $\mathrm{O}_{2}$. Being a spherical top, $\mathrm{SF}_{6}$ was immune to centrifuge spinning but caused the formation of a clearly visible plasma channel. As can be seen in Fig. 6.14 (b), when ionization was the dominant reason for the gas heating, an amplitude of the generated sound wave showed very different energy dependence than that observed in pure oxygen. Note that the stronger sound signal in plasma can be attributed to the higher number of ionized molecules in comparison to molecular super rotors. The non-ionizing mechanism of the recorded sound is also evident from the following test. We modified the laser field in such a way as to keep the direction of its vector of linear polarization constant, in contrast to the spinning polarization of the centrifuge pulse, while retaining the pulse duration and peak intensity. We observed that together with the forced accelerated molecular rotation, the acoustic signal disappeared 
beyond our sensitivity level.

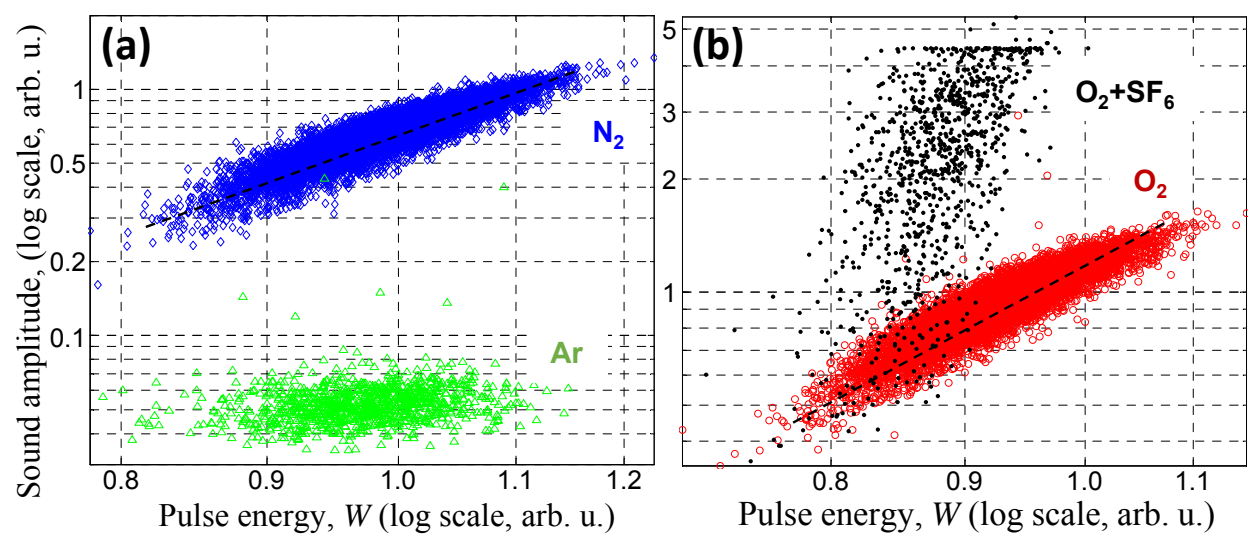

Figure 6.14: Amplitude of the recorded sound as a function of the energy of centrifuge pulses, plotted on a $\log$ - $\log$ scale. Each data set consists of 10,000 points. (a) Typical acoustic signal from the centrifuged gas of nitrogen molecules (blue diamonds) is compared to the sound generated by the centrifuge in pure argon at the same pressure of $95 \mathrm{kPa}$ (green triangles). (b) Acoustic response of the centrifuged oxygen with and without a small admixture of $\mathrm{SF}_{6}$ molecules (black dots and red circles, respectively). Black dashed lines in both panels show fits to power-law scaling.

Shown by black dashed lines in Fig. 6.14, an average sound amplitude from both nitrogen and oxygen super rotors exhibited power-law scaling with pulse energy. The exact power is not universal and typically varied between 4 and 5 in our experiments with nitrogen, and between 3 and 4 with oxygen. According to the theory of laser-induced pressure waves in dense media [44], as well as numerical simulations of hydrodynamic expansion [133], the sound wave amplitude is expected to scale linearly with the amount of laser energy deposited in the sample. In the case of an impulsive rotational excitation, most of the energy is transferred via a single two-photon Raman transition, suggesting a quadratic dependence on the pulse energy [59], which has been verified experimentally 65,141$]$. In contrast, the number of molecules, which initially occupied a particular rotational quantum state and were spun by the centrifuge, is proportional to the centrifuge volume. The latter can be estimated by the distance from the center of the laser beam, at which the optical potential of the centrifuge field is higher than the initial rotational energy. Since the induced-dipole potential is linear with the laser intensity, 
the volume will grow as its third power. Larger scaling power, observed in our experiments, can be attributed to the fact that together with the growing centrifuge volume for each trapped rotational state, the number of these states might have also increased with increasing laser intensity.

Because the efficiency of the centrifuge spinning depends on the molecular parameters, such as the moment of inertia and the polarizability anisotropy, this scaling is different for different molecules (compare $\mathrm{N}_{2}$ and $\mathrm{O}_{2}$ in panels (a) and (b) of Fig. 6.14, respectively). The scatter of data points within each set is due to the fluctuations of the spatial and spectral shape of centrifuge pulses, which caused shot-to-shot variations in the centrifuge efficiency.

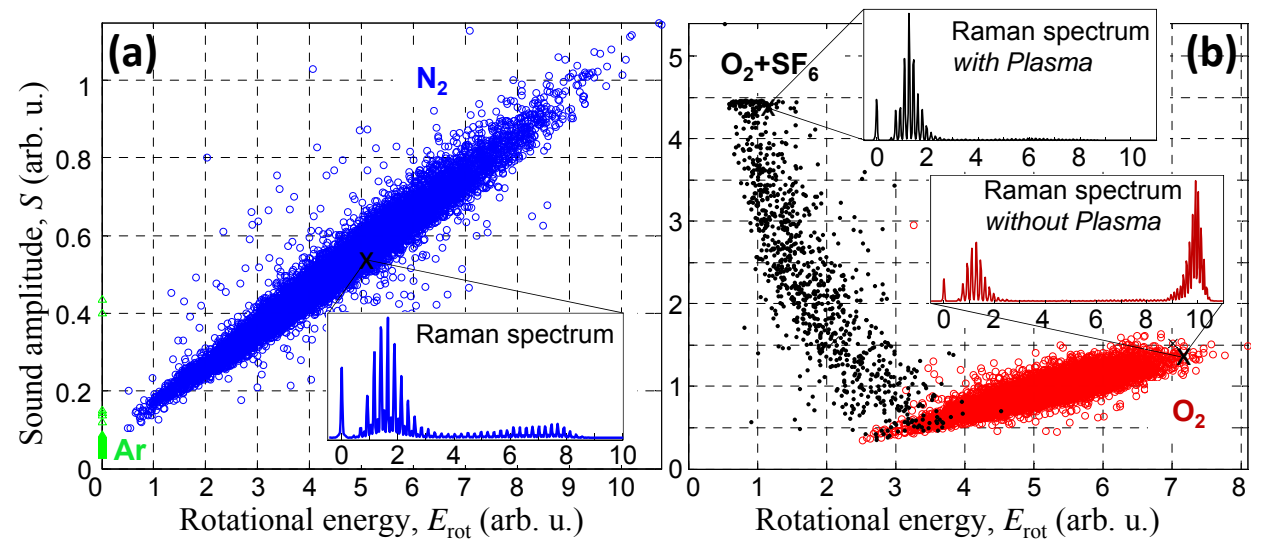

Figure 6.15: Amplitude of the recorded sound as a function of the rotational energy deposited in the gas sample. (a) Acoustic response from the centrifuged gas of nitrogen molecules (blue circles) is compared to the sound generated by the centrifuge in pure argon at the same pressure of $95 \mathrm{kPa}$ (green triangles). (b) Acoustic response from the centrifuged oxygen with and without a small admixture of $\mathrm{SF}_{6}$ molecules (black dots and red circles, respectively). All insets show Raman spectra corresponding to the data points marked with black crosses and plotted as a function of rotational frequency in $\mathrm{THz}$.

We eliminated the uncertainty in the degree of rotational excitation by sending a weak probe pulse behind each centrifuge pulse and recording the rotational Raman spectrum simultaneously with the corresponding photoacoustic signal. This enabled us to evaluate the amount of total rotational energy deposited in the molecular sample as a sum of rotational populations $P_{N}$ weighted with the corresponding rotational energy $E_{N}$ and taken over 
the rotational states $|N\rangle$ populated by the centrifuge:

$$
E_{\mathrm{rot}}=\sum_{N} P_{N} E_{N}
$$

A crude estimate of populations $P_{N}$ can be obtained from the measured Raman spectrum. Indeed, the intensity of each peak, $R_{N}$, is proportional to the square of the centrifuge-induced coherence $\rho_{N, N+2}$ between the two resonant states $|N\rangle$ and $|N+2\rangle$. For $N$ 's extending beyond the thermally populated states (i.e. $\gtrsim 20$ for $\mathrm{N}_{2}$ and $\gtrsim 30$ for $\mathrm{O}_{2}$ ), an optical centrifuge excites pure quantum states of maximum coherence, $\left|\rho_{N, N+2}\right|^{2}=P_{N} P_{N+2}$. If the created rotational wave packet is rather broad with a smoothly changing envelope, which was typically the case here, one may approximate $P_{N} \approx P_{N+2}$ and correspondingly $\left|\rho_{N, N+2}\right|^{2} \approx P_{N}^{2}$. Hence, the deposited rotational energy can be estimated from the measured Raman spectrum as

$$
E_{\mathrm{rot}} \approx \sum_{N>N_{\mathrm{th}}} \sqrt{R_{N}} E_{N}
$$

In Fig. 6.15, the dependence of the sound amplitude on the deposited rotational energy, extracted from the observed Raman spectra and calculated with Eq. (6.7), is shown for the same experimental runs as in Fig. 6.14. For nitrogen and oxygen super rotors (left and right panels, respectively), the scaling of the average photo-acoustic signal with $E_{\text {rot }}$ appears to be linear, in agreement with the hydrodynamic theory of heat transfer in dense media. The smooth envelope shape of the rotational wave packets in nitrogen, shown in the inset to Fig. 6.15 (a), together with the anticipated linear scaling of $S\left(E_{\text {rot }}\right)$, confirms the validity of the approximation we used to arrive at Eq. (6.7). In oxygen, the created wave packets are typically not as broad and smooth as in nitrogen (lower inset in Fig. 6.15 (b)), but the observed scaling is still linear with $E_{\text {rot }}$.

To emphasize the difference in the acoustic response in the presence of plasma, the same plot shows the experimentally detected distribution $S\left(E_{\text {rot }}\right)$ in the mixture of $\mathrm{O}_{2}$ with $\mathrm{SF}_{6}$. Whenever the plasma channel is formed, the wavefront of the centrifuge beam is perturbed, which leads to the loss of coherent rotational excitation and the disappearance of super rotors, as demonstrated by the Raman spectrum in the upper inset to Fig. 6.15 (b). An increase in the sound loudness is reflected by the observed anti-correlation between the two quantities. We also verified that including the low-frequency part of the Raman spectra, corresponding to hot molecules lost from the centrifuge, did not affect the observed linear 


\subsection{Sound emission from the gas of molecular super rotors}

scaling, most probably due to the small amount of rotational energy carried by those low- $N$ rotors. 


\section{Chapter 7}

\section{Outlook}

Optical centrifuge proved a remarkably powerful tool for controlling molecular rotation and studying molecules in previously inaccessible extreme rotational states. In this last chapter I will give a brief outlook on further developments and applications of the centrifuge technique and propose a few future experiments.

\subsection{Generation of $\mathrm{THz}$ radiation}

The flexibility of the centrifuge excitation, which allows one to control the rotational frequencies of molecules in a broad range from 0 to $10 \mathrm{THz}$, suggests a possibility for a novel way of generating tunable $\mathrm{THz}$ radiation. Indeed, a classical rotating dipole radiates at the frequency of its rotation. Quantum mechanically, by creating an isolated rotational wave packet (such as the one reflected by the Raman spectrum in Fig. 6.13), the centrifuge may not only populate high rotational states, but also create population inversion between them. Provided the transitions between the rotational levels are allowed, as they are in polar molecules, the system may serve either as a $\mathrm{THz}$ amplifier, or even a source of $\mathrm{THz}$ superradiance. The frequency of this $\mathrm{THz}$ radiation, determined by the rotational frequency of molecules, will be controllable with the centrifuge.

\subsection{Transient magnetization of paramagnetic gases}

As was shown in sections 4.4 and 5.2 , paramagnetic super rotors precess around the direction of external magnetic field, provided that the field strength is not too high. In this regime, the electronic spin $\mathbf{S}$ stays coupled to the angular momentum $\mathbf{N}$, which makes the precession frequency $\Omega$ depend on the sign of the projection $S_{N}$, as indicated by Eq.(4.7). It is this peculiar dependence on $S_{N}=0, \pm 1$ that explains the splitting of 


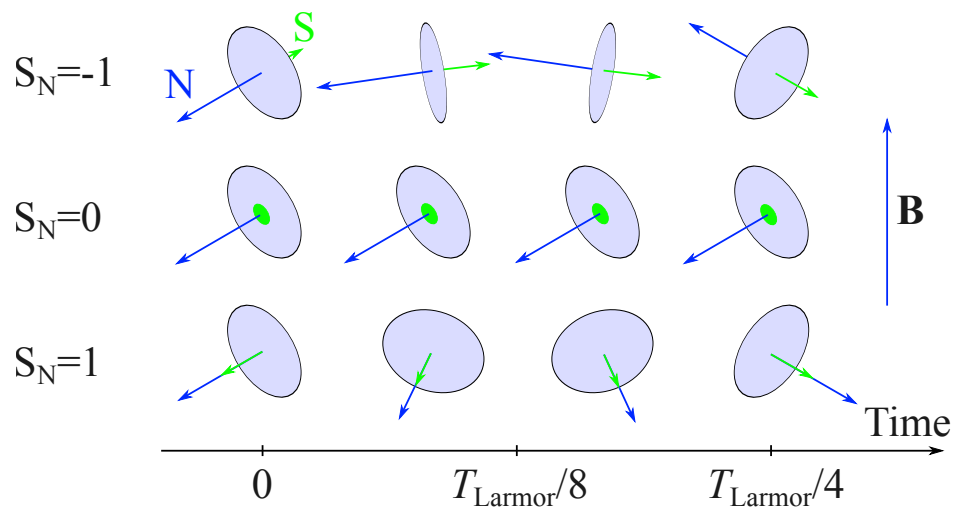

Figure 7.1: Precession of the angular distributions (blue disks) of paramagnetic spin-1 super rotors in magnetic field. Different rows correspond to different spin projections $S_{N}$. Blue and green arrows represent the angular momentum and spin vectors, respectively.

an isotropic molecular distribution into three parts, observed in our experiments and discussed in section 4.4. As illustrated in Fig. 7.1, the two counter-rotating discs, corresponding to $S_{N}= \pm 1$, meet at exactly one quarter of the Larmor period. At this time, the two initially opposite projections of the electronic spin become the same, causing a macroscopic magnetization of the gas. Notice, that no initial spin polarization of molecules, but rather their centrifuge-induced planar alignment, is required for this effect. Because the magnetization is produced by a rather large number of molecules with high magnetic moment of $\mu_{B}$, its magnitude could be quite high and potentially detectable by a small pick-up coil.

\subsection{Field-free alignment of asymmetric top molecules}

Achieving field-free alignment of asymmetric top molecules is a hard task due to the complicated dynamics they exhibit. As was shown in section 5.3, strong planar alignment due to the centrifugal forces reduces the dynamics of asymmetric tops to that of a simple linear rotor. The latter are routinely aligned using non-adiabatic methods, e.g. by a strong femtosecond pulse. Indeed, the low recurring 3D alignment, demonstrated in Fig. 5.13 (a) was a consequence of the weak non-adiabaticity of the centrifuge excitation. 
This non-adiabatic part can be substantially enhanced by adding a kick excitation after the centrifuge, producing much higher degrees of transient three-dimensional alignment.

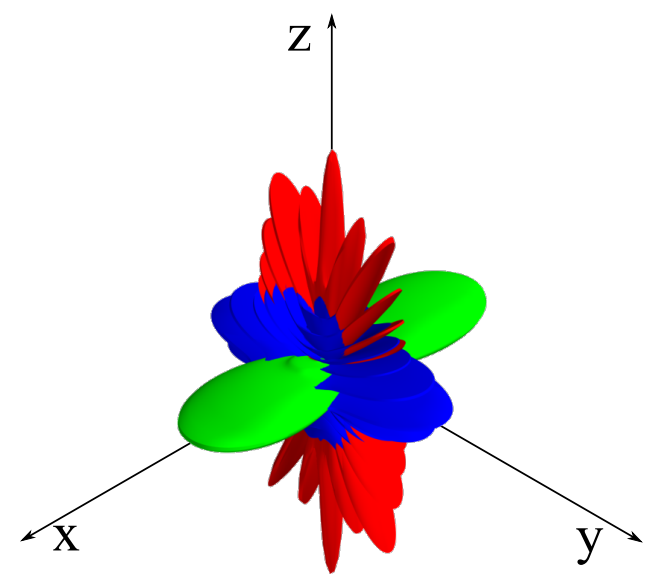

Figure 7.2: Calculated distributions of molecular axes $a$ (red), $b$ (green) and $c$ (blue) of an asymmetric top molecule in the aligned rotational state.

A fully adiabatic field-free technique is even more desirable, since it produces permanent molecular alignment. The two ingredients are vital to the development of such methods: the stationary rotational states that posses some degree of 3D alignment, and the adiabatic way of populating these states. Our preliminary calculations confirm the existence of such 3D aligned stationary states of asymmetric top. An example of one such state,

$$
\sum_{M_{J} \in\{-2,0,2\}} \frac{1}{\sqrt{3}}\left|J=16, M_{J}, \tau=-12\right\rangle,
$$

is illustrated in Fig. 7.2 by showing the distributions of molecular axes $a$ (red), $b$ (green) and $c$ (blue). As can be seen, it indeed exhibits a small degree of permanent 3D alignment. As was demonstrated in section 5.1, changing the behavior of the centrifuge polarization may offer control over the target states of the centrifuge excitation, while keeping it adiabatic. Another degree of control could be introduced by combining several centrifuge pulses one after another, potentially allowing reaching the desired 3D aligned state via a few consecutive adiabatic transfers. The feasibility of this method requires further study. 


\subsection{Potential energy surface reconstruction}

Potential energy surface (PES) is one of the most important characteristics of the molecule. It determines how the potential energy $V$ of the molecular bonds depends on the intramolecular coordinates $\mathbf{r}$. A conventional way of finding $V(\mathbf{r})$ involves fitting a particular molecule-specific model potential to the experimentally-measured vibrational spectrum. At large $\mathbf{r}$, such fitting requires the the knowledge of high vibrational states.

An optical centrifuge could provide an additional information on PES. As was shown in section 5.3, fast rotation deforms the molecule and modifies its field-free dynamics. Experimentally, the revival time $T_{\text {rev }}$ can be determined as a function of the rotational frequency $\omega$. Since $T_{\mathrm{rev}}=\frac{h}{d^{2} \epsilon_{J} / d J^{2}}$ and $\omega=\frac{1}{\hbar} \frac{d \epsilon_{J}}{d J}$, where $\epsilon_{J}$ is the energy of the state $J$, we can write $T_{\text {rev }}(\omega)=\frac{2 \pi}{d \omega / d J}$ and use

$$
2 \pi J=\int T_{\mathrm{rev}}(\omega) d \omega
$$

to determine $\omega(J)$. Integrating this function one more time, we achieve the rotational spectrum $\epsilon_{J}$. Our preliminary results show, that the bond deformations at the highest achievable $J$-states become compatible to or even higher than what can be reached through pure vibrational excitation. This means, that fitting $\epsilon_{J}$ by the model potential at the extreme level of rotational excitation may improve the knowledge of $V(\mathbf{r})$ at large $\mathbf{r}$.

\subsection{Harmonic rotational states}

As the angular momentum of a linear molecule increases in the centrifuge, it starts stretching. This leads to the increase of its moment of inertia, which in turn may result in a peculiar situation, when the rotational frequency $\omega(J)=\frac{J}{I(J)}$ stops increasing with angular momentum $J$. Our preliminary calculations and experimental results point to the existence of this effect in a number of linear molecules.

Since $\omega=\frac{1}{\hbar} \frac{d \epsilon_{J}}{d J}$, near this angular velocity the spectrum becomes equidistant, making the system equivalent to the quantum harmonic oscillator. Among others, one of the interesting expected features of such "harmonic" rotational states is infinitely large revival time. This means, that even a narrowly localized rotational wave packet would undergo dispersionless classical-like motion indefinitely. 


\subsection{Super rotors in He droplets}

A lot of attention has recently been attracted to the behavior of molecules embedded in helium nanodroplets [124]. Modern technology allows creation of small (from a few hundred to a few million of atoms) helium clusters with exactly one molecule embedded inside. Due to the low polarizability and high ionization threshold of such helium clusters, an embedded molecule remains accessible to the rotational excitation by laser fields [16, 97]. As recent studies showed [124], the rotational states of embedded molecules exhibit surprisingly low, for a condensed phase, decoherence rate, which was attributed to the superfluidity of helium clusters below the lambda point. These studies, however, were limited by a relatively low degree of rotational excitation and wave packet controllability.

It is therefore of great interest to extend our studies of the collisional decay of super rotors (chapter 6) to He nanodroplets. Studying the decoherence rates as a function of the molecular angular momentum inside a quantum environment of a nanodroplet could provide better understanding of the decoherence mechanisms in the many-body quantum interaction limit. Unlike macroscopic gas samples investigated in this thesis, the thermalization volume of a nanodroplet is rather small, which could offer opportunities for exciting collective angular momentum states, such as quantum vortices [41]. 


\section{Chapter 8}

\section{Conclusions}

In this work we implemented the technique of an optical centrifuge to produce and study the unique states of molecules, molecular super rotors. Several experimental techniques were developed to detect and characterize super rotors of various molecular species in the presence of external fields and under field-free conditions, in dilute gas samples and in dense medium.

We showed, that the centrifuge offered high degree of control over the rotational state of molecules, allowing an excitation of synchronous unidirectional rotation in a very broad range of frequencies. Finite width of the produced wave packet allowed us to observe novel rotational and spinrotational dynamics. Our studies of the evolution of super rotors in external magnetic fields provided a new way of controlling their rotational states.

We proposed and implemented a number of experimental techniques that employed an optical centrifuge to achieve the molecular alignment at high degrees of rotational excitation. Permanent field-free planar alignment of asymmetric top molecules was demonstrated for the first time. The proposed methods could open new possibilities in achieving high degrees of field-free 3D alignment of such systems.

Experiments in dense gases allowed us to study the processes of collisional relaxation of molecular super rotors. All expected stages of this relaxation were observed and identified experimentally, providing the existing theories of microscopic molecular dissipation with a much broader testing ground. We showed, that high rotational excitation suppresses decoherence and makes super rotors extremely robust to collisions. Even after initial rotational dephasing, they were found to preserve their rotational directionality for a long time, allowing us to produce ensembles of molecules with long-lived field-free alignment. Extending these studies to quantum environments, such as He nanodroplets, represents an exciting new frontier of molecular science. 


\section{Bibliography}

[1] M. Artamonov and T. Seideman. Theory of three-dimensional alignment by intense laser pulses. J. Chem. Phys., 128(15):154313, 2008. ISSN 00219606. doi: 10.1063/1.2894876. URL http://scitation. aip.org/content/aip/journal/jcp/128/15/10.1063/1.2894876.

[2] M. N. R. Ashfold and J. D. Howe. Multiphoton Spectroscopy of Molecular Species. Annu. Rev. Phys. Chem., 45(1):57-82, oct 1994. ISSN 0066-426X. doi: 10.1146/annurev.pc.45.100194.000421. URL http://www. annualreviews.org/doi/abs/10.1146/annurev. pc.45.100194.000421

[3] M. N. R. Ashfold, J. Bayley, and R. Dixon. Molecular predissociation dynamics revealed through multiphoton ionisation spectroscopy. I. The 1B1 states of $\mathrm{H} 2 \mathrm{O}$ and D2O. Chem. Phys., 84(1):35-50, feb 1984. ISSN 03010104. doi: 10.1016/0301-0104(84)80004-X. URL http: //linkinghub.elsevier.com/retrieve/pii/030101048480004X.

[4] A. V. Avdeenkov and J. L. Bohn. Ultracold collisions of oxygen molecules. Phys. Rev. A, 64(5):052703, oct 2001. ISSN 1050-2947. doi: 10.1103/PhysRevA.64.052703. URL http://arxiv.org/abs/physics/0105085http://link.aps.org/ doi/10.1103/PhysRevA.64.052703.

[5] I. S. Averbukh and N. Perelman. Fractional revivals: Universality in the long-term evolution of quantum wave packets beyond the correspondence principle dynamics. Phys. Lett. A, 139(9): 449-453, aug 1989. ISSN 03759601. doi: 10.1016/0375-9601(89) 90943-2. URL http://www.sciencedirect.com/science/article/ pii/0375960189909432.

[6] I. S. Averbukh and N. Perel'man. The dynamics of wave packets of highly-excited states of atoms and molecules. Uspekhi Fiz. Nauk, 161 (7):41, 1991. ISSN 0042-1294. doi: 10.3367/UFNr.0161.199107b.0041. URL http://ufn.ru/ru/articles/1991/7/b/. 
[7] M. Berard. Experimental and theoretical analysis of the temperature dependence of rotational Raman linewidths of oxygen. $J$. Chem. Phys., 78(2):672, 1983. ISSN 00219606. doi: 10.1063/1. 444811. URL http://scitation.aip.org/content/aip/journal/ jcp/78/2/10.1063/1.444811.

[8] R. Beringer and J. G. Castle. Microwave Magnetic Resonance Spectrum of Oxygen. Phys. Rev., 81(1):82-88, jan 1951. ISSN 0031-899X. doi: 10.1103/PhysRev.81.82. URL http://link.aps.org/doi/10. 1103/PhysRev.81.82.

[9] R. J. Beuhler, R. B. Bernstein, and K. H. Kramer. Observation of the Reactive Asymmetry of Methyl Iodide. Crossed Beam Study of the Reaction of Rubidium with Oriented Methyl Iodide Molecules. J. Am. Chem. Soc., 88(22):5331-5332, nov 1966. ISSN 0002-7863. doi: 10.1021/ja00974a059. URL http://pubs .acs.org/doi/abs/10. 1021/ja00974a059.

[10] P. R. Brooks. Reactive Scattering of K Atoms from Oriented CH3I Molecules. J. Chem. Phys., 45(9):3449, 1966. ISSN 00219606. doi: 10.1063/1.1728128. URL http://scitation.aip.org/content/ aip/journal/jcp/45/9/10.1063/1.1728128.

[11] J. M. Brown and A. Carrington. Rotational Spectroscopy of Diatomic Molecules. Cambridge University Press, Cambridge, 2003. ISBN 9780511814808. doi: 10.1017/CBO9780511814808. URL http: //ebooks.cambridge.org/ref/id/CB09780511814808.

[12] T. A. Brunner. Rotational energy transfer in Na2 (A $\Sigma$ ) colliding with $\mathrm{Xe}, \mathrm{Kr}, \mathrm{Ar}, \mathrm{Ne}, \mathrm{He}, \mathrm{H} 2, \mathrm{CH} 4$, and N2: Experiment and fitting laws. J. Chem. Phys., 74(6):3324, 1981. ISSN 00219606. doi: 10.1063/1. 441485. URL http://scitation.aip.org/content/aip/journal/ jcp/74/6/10.1063/1.441485.

[13] T. A. Brunner, R. D. Driver, N. Smith, and D. E. Pritchard. Rotational energy transfer in Na2Xe collisions: Level to level dynamics. J. Chem. Phys., 70(9):4155, 1979. ISSN 00219606. doi: 10.1063/1.438040. URL http://scitation.aip.org/content/aip/ journal/jcp/70/9/10.1063/1.438040.

[14] A. Buchleitner and D. Delande. Nondispersive Electronic Wave Packets in Multiphoton Processes. Phys. Rev. Lett., 75(8):1487-1490, aug 
1995. ISSN 0031-9007. doi: 10.1103/PhysRevLett.75.1487. URL http://link.aps.org/doi/10.1103/PhysRevLett.75.1487.

[15] Y.-H. Cheng, J. K. Wahlstrand, N. Jhajj, and H. M. Milchberg. The effect of long timescale gas dynamics on femtosecond filamentation. Opt. Express, 21(4):4740, feb 2013. ISSN 1094-4087. doi: 10.1364/OE.21.004740. URL https://www.osapublishing.org/oe/ abstract.cfm?uri=oe-21-4-4740.

[16] L. Christiansen, J. H. Nielsen, D. Pentlehner, J. G. Underwood, and H. Stapelfeldt. Alignment enhancement of molecules embedded in helium nanodroplets by multiple laser pulses. Phys. Rev. A, 92 (5):053415, nov 2015. ISSN 1050-2947. doi: 10.1103/PhysRevA. 92.053415. URL http://link.aps.org/doi/10.1103/PhysRevA.92. 053415 .

[17] J. P. Cryan, P. H. Bucksbaum, and R. N. Coffee. Field-free alignment in repetitively kicked nitrogen gas. Phys. Rev. A, 80(6):063412, dec 2009. ISSN 1050-2947. doi: 10.1103/PhysRevA.80.063412. URL http: //link.aps.org/doi/10.1103/PhysRevA.80.063412.

[18] J. P. Cryan, J. M. Glownia, D. W. Broege, Y. Ma, and P. H. Bucksbaum. Ensemble of Linear Molecules in Nondispersing Rotational Quantum States: A Molecular Stopwatch. Phys. Rev. X, 1(1):011002, aug 2011. ISSN 2160-3308. doi: 10.1103/PhysRevX.1.011002. URL http://link.aps.org/doi/10.1103/PhysRevX.1.011002.

[19] A. Cuisset, O. Pirali, and D. a. Sadovskií. Gyroscopic Destabilization of Molecular Rotation and Quantum Bifurcation Observed in the Structure of the $<$ math display $=$ "inline" $><$ msub $><\operatorname{mi}>\nu</ \mathrm{mi}>$ $<\mathrm{mn}>23</ \mathrm{mn}></$ msub $></$ math $>$ Fundamental of Dimethylsulfoxyde. Phys. Rev. Lett., 109(9):094101, aug 2012. ISSN 0031-9007. doi: 10.1103/PhysRevLett.109.094101. URL http://link.aps.org/ doi/10.1103/PhysRevLett.109.094101.

[20] S. M. Cybulski and D. M. Bishop. Calculations of magnetic properties. IV. Electron-correlated magnetizabilities and rotational g factors for nine small molecules. J. Chem. Phys., 100(3):2019, 1994. ISSN 00219606. doi: 10.1063/1.467234. URL http://scitation.aip.org/ content/aip/journal/jcp/100/3/10.1063/1.467234

[21] J. Czarny, P. Felenbok, and H. Lefebvre-Brion. High vibrational level predissociation in the A $2 \Sigma+$ state of OD. J. Phys. B At. Mol. Phys., 
4(1):124-132, jan 1971. ISSN 0022-3700. doi: 10.1088/0022-3700/4/1/ 017. URL http://stacks.iop.org/0022-3700/4/i=1/a=017?key= crossref.e6fb15960b562b30b596c8e85ce17a8c.

[22] A. E. DePristo, S. D. Augustin, R. Ramaswamy, and H. Rabitz. Quantum number and energy scaling for nonreactive collisions. $J$. Chem. Phys., 71(2):850, 1979. ISSN 00219606. doi: 10.1063/1. 438376. URL http://scitation.aip.org/content/aip/journal/ jcp/71/2/10.1063/1.438376.

[23] B. Dick. Inverting ion images without Abel inversion: maximum entropy reconstruction of velocity maps. Phys. Chem. Chem. Phys., 16(2):570-580, 2014. ISSN 1463-9076. doi: 10. 1039/C3CP53673D. URL http://www.ncbi.nlm.nih.gov/pubmed/ 24172596http://xlink.rsc.org/?DOI=C3CP53673D.

[24] G. H. Dieke and H. D. Babcock. The structure of the atmospheric absorption bands of oxygen. Proc. Natl. Acad. Sci., 13:670-678, 1927.

[25] P. Dooley, I. Litvinyuk, K. Lee, D. Rayner, M. Spanner, D. Villeneuve, and P. B. Corkum. Direct imaging of rotational wave-packet dynamics of diatomic molecules. Phys. Rev. A, 68(2):023406, aug 2003. ISSN 1050-2947. doi: 10.1103/PhysRevA.68.023406. URL http://link. aps.org/doi/10.1103/PhysRevA.68.023406.

[26] V. Dribinski, A. Ossadtchi, V. a. Mandelshtam, and H. Reisler. Reconstruction of Abel-transformable images: The Gaussian basis-set expansion Abel transform method. Rev. Sci. Instrum., 73(7):2634, 2002. ISSN 00346748. doi: 10.1063/1.1482156. URL http://scitation. aip.org/content/aip/journal/rsi/73/7/10.1063/1.1482156.

[27] C. Ellert and P. B. Corkum. Disentangling molecular alignment and enhanced ionization in intense laser fields. Phys. Rev. A, 59(5):R3170R3173, may 1999. ISSN 1050-2947. doi: 10.1103/PhysRevA.59.R3170. URL http://journals.aps.org/pra/abstract/10.1103/ PhysRevA.59.R3170\$\delimiter"026E30F\$nhttp://link.aps . org/doi/10.1103/PhysRevA.59.R3170http://link.aps.org/doi/ 10.1103/PhysRevA.59.R3170.

[28] S. Fleischer, I. S. Averbukh, and Y. Prior. Selective alignment of molecular spin isomers. Phys. Rev. Lett., 99(9):1-4, 2007. ISSN 00319007. doi: 10.1103/PhysRevLett.99.093002. 
[29] S. Fleischer, Y. Khodorkovsky, Y. Prior, and I. S. Averbukh. Controlling the sense of molecular rotation. New J. Phys., 11(10):105039, oct 2009. ISSN 1367-2630. doi: 10.1088/1367-2630/11/10/105039. URL http://stacks . iop.org/1367-2630/11/i=10/a=105039?key= crossref.70b99529a0204cfe183fa7e7b85dc3c5.

[30] S. Fleischer, Y. Khodorkovsky, E. Gershnabel, Y. Prior, and I. S. Averbukh. Molecular Alignment Induced by Ultrashort Laser Pulses and Its Impact on Molecular Motion. Isr. J. Chem., 52(5):414-437, may 2012. ISSN 00212148. doi: 10.1002/ijch.201100161. URL http: //doi.wiley.com/10.1002/ijch.201100161.

[31] J. Floß. A theoretical study of the dynamics of paramagnetic superrotors in external magnetic fields. J. Phys. B At. Mol. Opt. Phys., 48(16):164005, aug 2015. ISSN 0953-4075. doi: 10.1088/0953-4075/ 48/16/164005. URL http://stacks.iop.org/0953-4075/48/i=16/ $\mathrm{a}=164005$ ?key=crossref $.153 \mathrm{c} 3945 \mathrm{ec} 2131 \mathrm{~b} 6 \mathrm{a} 5 \mathrm{c} 276980 \mathrm{bf} 00 \mathrm{aba}$.

[32] W. H. Flygare and R. Benson. The molecular Zeeman effect in diamagnetic molecules and the determination of molecular magnetic moments ( $\mathrm{g}$ values), magnetic susceptibilities, and molecular quadrupole moments. Mol. Phys., 20(2):225-250, jan 1971. ISSN 0026-8976. doi: 10.1080/00268977100100221. URL http://www.tandfonline.com/ doi/abs/10.1080/00268977100100221.

[33] R. C. Forrey. Prospects for cooling and trapping rotationally hot molecules. Phys. Rev. A, 66(2):023411, aug 2002. ISSN 1050-2947. doi: 10.1103/PhysRevA.66.023411. URL http://link.aps.org/doi/10. 1103/PhysRevA.66.023411.

[34] L. J. Frasinski, K. Codling, P. Hatherly, J. Barr, I. N. Ross, and W. T. Toner. Femtosecond dynamics of multielectron dissociative ionization by use of a picosecond laser. Phys. Rev. Lett., 58(23):2424-2427, jun 1987. ISSN 0031-9007. doi: 10.1103/PhysRevLett.58.2424. URL http://link.aps.org/doi/10.1103/PhysRevLett.58.2424.

[35] H.-M. Frey, P. Beaud, T. Gerber, B. Mischler, P. Radi, and A. Tzannis. Femtosecond nonresonant degenerate four-wave mixing at atmospheric pressure and in a free jet. Appl. Phys. B, 68(4):735-739, apr 1999. ISSN 0946-2171. doi: 10.1007/s003400050696. URL http://link. springer.com/10.1007/s003400050696. 
[36] B. Friedrich and D. Herschbach. Alignment and trapping of molecules in intense laser fields. Phys. Rev. Lett., 74(23):4623-4626, jun 1995. ISSN 1079-7114. URL http://www.ncbi.nlm.nih.gov/pubmed/ 10058557 .

[37] B. Friedrich and D. R. Herschbach. On the possibility of aligning paramagnetic molecules or ions in a magnetic field. Zeitschrift fur Phys. D Atoms, Mol. Clust., 24(1):25-31, mar 1992. ISSN 0178-7683. doi: 10.1007/BF01436600. URL http://link.springer.com/10.1007/ BF01436600.

[38] G. A. Garcia, L. Nahon, and I. Powis. Two-dimensional charged particle image inversion using a polar basis function expansion. Rev. Sci. Instrum., 75(11):4989, 2004. ISSN 00346748. doi: 10.1063/1. 1807578. URL http://scitation.aip.org/content/aip/journal/ rsi/75/11/10.1063/1.1807578.

[39] E. Gershnabel and I. S. Averbukh. Deflection of Field-Free Aligned Molecules. Phys. Rev. Lett., 104(15):153001, apr 2010. ISSN 00319007. doi: 10.1103/PhysRevLett.104.153001. URL http://link. aps.org/doi/10.1103/PhysRevLett.104.153001.

[40] E. Gershnabel, M. Shapiro, and I. S. Averbukh. Stern-Gerlach deflection of field-free aligned paramagnetic molecules. J. Chem. Phys., 135(19):194310, 2011. ISSN 00219606. doi: 10.1063/1.3662135. URL http://scitation.aip.org/content/aip/journal/jcp/135/ 19/10.1063/1.3662135.

[41] L. F. Gomez, K. R. Ferguson, J. P. Cryan, C. Bacellar, R. M. P. Tanyag, C. Jones, S. Schorb, D. Anielski, A. Belkacem, C. Bernando, R. Boll, J. Bozek, S. Carron, G. Chen, T. Delmas, L. Englert, S. W. Epp, B. Erk, L. Foucar, R. Hartmann, A. Hexemer, M. Huth, J. Kwok, S. R. Leone, J. H. S. Ma, F. R. N. C. Maia, E. Malmerberg, S. Marchesini, D. M. Neumark, B. Poon, J. Prell, D. Rolles, B. Rudek, A. Rudenko, M. Seifrid, K. R. Siefermann, F. P. Sturm, M. Swiggers, J. Ullrich, F. Weise, P. Zwart, C. Bostedt, O. Gessner, and A. F. Vilesov. Shapes and vorticities of superfluid helium nanodroplets. Science (80-. )., 345(6199):906-909, aug 2014. ISSN 00368075. doi: 10.1126/science.1252395. URL http://www.sciencemag. org/cgi/doi/10.1126/science.1252395 
[42] J.-M. Hartmann and C. Boulet. Quantum and classical approaches for rotational relaxation and nonresonant laser alignment of linear molecules: A comparison for $\mathrm{CO} 2$ gas in the nonadiabatic regime. $J$. Chem. Phys., 136(18):184302, may 2012. ISSN 00219606. doi: 10. 1063/1.4705264. URL http://scitation.aip.org/content/aip/ journal/jcp/136/18/10.1063/1.4705264.

[43] R. Hasbani, B. Ostojić, P. R. Bunker, and M. Y. Ivanov. Selective dissociation of the stronger bond in HCN using an optical centrifuge. J. Chem. Phys., 116:10636, 2002. URL http://link.aip.org/link/ JCPSA6/v116/i24/p10636/s1.

[44] J.-M. Heritier. Electrostrictive limit and focusing effects in pulsed photoacoustic detection. Opt. Commun., 44(4):267-272, jan 1983. ISSN 00304018. doi: 10.1016/0030-4018(83)90135-9. URL http: //linkinghub.elsevier.com/retrieve/pii/0030401883901359.

[45] G. Herzberg. Molecular spectra and molecular structure, volume I. Krieger publishing co, Malabar, Florida, 2nd edition, 1950.

[46] G. Herzberg. Molecular Spectra \& Molecular Structure III ( Polyatomic Molecules ). D. Van Nostrand Company, Inc., Princeton, New Jersey, 1966.

[47] G. Herzberg. Molecular Spectra and Molecular Structure: Spectra of diatomic molecules. Electronic Spectra and Electronic Structure of Polyatomic Molecules. R.E. Krieger Publishing Company, 1989. ISBN 9780894642685. URL https://books.google.ca/books?id= ees9AQAAIAAJ.

[48] A. Hishikawa, A. Iwamae, K. Hoshina, M. Kono, and K. Yamanouchi. Mass-resolved two-dimensional momentum imaging of the Coulomb explosion of $\mathrm{N} 2$ and $\mathrm{SO} 2$ in an intense laser field. Chem. Phys. Lett., 282(3-4):283-291, jan 1998. ISSN 00092614. doi: 10.1016/ S0009-2614(97)01325-0. URL http://linkinghub.elsevier.com/ retrieve/pii/S0009261497013250.

[49] M. Z. Hoque, M. Lapert, E. Hertz, F. Billard, D. Sugny, B. Lavorel, and O. Faucher. Observation of laser-induced field-free permanent planar alignment of molecules. Phys. Rev. A, 84(1):013409, jul 2011. ISSN 1050-2947. doi: 10.1103/PhysRevA.84.013409. URL http:// link.aps.org/doi/10.1103/PhysRevA.84.013409. 
[50] J. Houzet, J. Gateau, E. Hertz, F. Billard, B. Lavorel, J.-M. Hartmann, C. Boulet, and O. Faucher. Probing ultrafast thermalization with field-free molecular alignment. Phys. Rev. A, 86(3):033419, sep 2012. ISSN 1050-2947. doi: 10.1103/PhysRevA.86.033419. URL http://link.aps.org/doi/10.1103/PhysRevA.86.033419.

[51] K. P. Huber and G. Herzberg. NIST Chemistry WebBook, NIST Standard Reference Database, volume 69 of Constants of Diatomic Molecules. National Institute of Standards and Technology, Gaithersburg MD, 2012.

[52] W. Huttner and W. H. Flygare. Magnetic-Field Interactions in a Rotating Molecule. J. Chem. Phys., 47(10):4137, 1967. ISSN 00219606. doi: 10.1063/1.1701590. URL http://scitation.aip.org/content/ aip/journal/jcp/47/10/10.1063/1.1701590.

[53] J. Itatani, J. Levesque, D. Zeidler, H. Niikura, H. Pépin, J. C. Kieffer, P. B. Corkum, and D. M. Villeneuve. Tomographic imaging of molecular orbitals. Nature, 432(7019):867-871, 2004. URL http://www. nature.com/nature/journal/v432/n7019/abs/nature03183.html.

[54] J. Itatani, D. Zeidler, J. Levesque, M. Spanner, D. Villeneuve, and P. B. Corkum. Controlling High Harmonic Generation with Molecular Wave Packets. Phys. Rev. Lett., 94(12):123902, mar 2005. ISSN 00319007. doi: 10.1103/PhysRevLett.94.123902. URL http://link.aps. org/doi/10.1103/PhysRevLett.94.123902.

[55] P. W. Joireman, L. L. Connell, S. M. Ohline, and P. M. Felker. Characterization of asymmetry transients in rotational coherence spectroscopy. J. Chem. Phys., 96(6):4118, 1992. ISSN 00219606. doi: 10.1063/1.461867. URL http://scitation.aip.org/content/aip/ journal/jcp/96/6/10.1063/1.461867.

[56] M. Kalinski and J. H. Eberly. New States of Hydrogen in a Circularly Polarized Electromagnetic Field. Phys. Rev. Lett., 77(12):2420-2423, sep 1996. ISSN 0031-9007. doi: 10.1103/PhysRevLett.77.2420. URL http://link.aps.org/doi/10.1103/PhysRevLett.77.2420.

[57] J. Karczmarek, J. Wright, P. B. Corkum, and M. Ivanov. Optical Centrifuge for Molecules. Phys. Rev. Lett., 82(17):3420-3423, apr 1999. ISSN 0031-9007. doi: 10.1103/PhysRevLett.82.3420. URL http://link.aps.org/doi/10.1103/PhysRevLett.82.3420. 
[58] G. Karras, E. Hertz, F. Billard, B. Lavorel, J.-M. Hartmann, and O. Faucher. Using molecular alignment to track ultrafast collisional relaxation. Phys. Rev. A, 89(6):063411, jun 2014. ISSN 1050-2947. doi: 10.1103/PhysRevA.89.063411. URL http://link.aps.org/doi/10. 1103/PhysRevA.89.063411.

[59] D. V. Kartashov, a. V. Kirsanov, a. M. Kiselev, a. N. Stepanov, N. N. Bochkarev, Y. N. Ponomarev, and B. a. Tikhomirov. Nonlinear absorption of intense femtosecond laser radiation in air. Opt. Express, 14(17):7552, 2006. ISSN 1094-4087. doi: 10.1364/OE.14. 007552. URL https://www.osapublishing.org/oe/abstract.cfm? uri=oe-14-17-7552.

[60] S. P. Kearney, D. J. Scoglietti, and J. Kliewer. Hybrid femtosecond/picosecond rotational coherent anti-Stokes Raman scattering temperature and concentration measurements using two different picosecond-duration probes. Opt. Express, 21(10):12327, may 2013. ISSN 1094-4087. doi: 10.1364/OE.21.012327. URL https://www. osapublishing.org/oe/abstract.cfm?uri=oe-21-10-12327.

[61] Y. Khodorkovsky, I. S. Averbukh, and E. Pollak. Classical theory of rotational rainbow scattering from uncorrugated surfaces. J. Phys. Condens. Matter, 22(30):304004, aug 2010. ISSN 0953-8984. doi: 10.1088/0953-8984/22/30/304004. URL http://stacks . iop . org/0953-8984/22/i=30/a=304004?key= crossref.2e6a1e6063bfe727c93af71aa64aa262.

[62] Y. Khodorkovsky, U. Steinitz, J.-M. Hartmann, and I. S. Averbukh. Collisional dynamics in a gas of molecular super-rotors. Nat. Commun., 6(Umr 7583):7791, jul 2015. ISSN 2041-1723. doi: 10.1038/ ncomms8791. URL http://www.nature.com/doifinder/10.1038/ ncomms8791.

[63] W. Kim and P. M. Felker. Spectroscopy of pendular states in opticalfield-aligned species. J. Chem. Phys., 104(3):1147, $1996 . \quad$ ISSN 00219606. doi: 10.1063/1.470770. URL http://scitation.aip.org/ content/aip/journal/jcp/104/3/10.1063/1.470770

[64] W. Kim and P. M. Felker. Ground-state intermolecular spectroscopy and pendular states in benzeneargon. J. Chem. Phys., 107(7):2193, 1997. ISSN 00219606. doi: 10.1063/1.474599. 
URL http://scitation.aip.org/content/aip/journal/jcp/107/ 7/10.1063/1.474599.

[65] A. M. Kiselev, Y. N. Ponomarev, A. N. Stepanov, A. B. Tikhomirov, and B. A. Tikhomirov. Nonlinear absorption of femtosecond laser pulses $(800 \mathrm{~nm})$ by atmospheric air and water vapour. Quantum Electron., 41(11):976, 2011. URL http://stacks.iop.org/1063-7818/ $41 / i=11 / a=A 05$.

[66] K. Kitano, H. Hasegawa, and Y. Ohshima. Ultrafast Angular Momentum Orientation by Linearly Polarized Laser Fields. Phys. Rev. Lett., 103(22):223002, nov 2009. ISSN 0031-9007. doi: 10. 1103/PhysRevLett.103.223002. URL http://link . aps .org/doi/10. 1103/PhysRevLett.103.223002.

[67] C. J. Kliewer, A. Bohlin, E. Nordström, B. D. Patterson, P.-E. Bengtsson, and T. B. Settersten. Time-domain measurements of S-branch N2N2 Raman linewidths using picosecond pure rotational coherent anti-Stokes Raman spectroscopy. Appl. Phys. B, 108(2):419-426, aug 2012. ISSN 0946-2171. doi: 10.1007/s00340-012-5037-2. URL http://link.springer.com/10.1007/s00340-012-5037-2.

[68] O. Korech, U. Steinitz, R. J. Gordon, I. S. Averbukh, and Y. Prior. Observing molecular spinning via the rotational Doppler effect. Nat. Photonics, 7(9):711-714, jul 2013. ISSN 1749-4885. doi: 10.1038/ nphoton.2013.189. URL http://www.nature.com/doifinder/10. 1038/nphoton.2013.189.

[69] A. Korobenko, A. A. Milner, and V. Milner. Direct Observation, Study, and Control of Molecular Superrotors. Phys. Rev. Lett., 112(11):113004, mar 2014. ISSN 0031-9007. doi: 10. 1103/PhysRevLett.112.113004. URL http://link . aps . org/doi/10. 1103/PhysRevLett.112.113004.

[70] A. Korobenko, J. W. Hepburn, and V. Milner. Observation of nondispersing classical-like molecular rotation. Phys. Chem. Chem. Phys., 17 (2):951-956, 2015. ISSN 1463-9076. doi: 10.1039/C4CP04434G. URL http://xlink.rsc.org/?DOI=C4CP04434G.

[71] M. L. Koszykowski, L. A. Rahn, R. E. Palmer, and M. E. Coltrin. Theoretical and experimental studies of high-resolution inverse Raman spectra of molecular nitrogen at 1-10 atm. J. Phys. Chem., 91(1): 
41-46, jan 1987. ISSN 0022-3654. doi: 10.1021/j100285a012. URL http://pubs.acs.org/doi/abs/10.1021/j100285a012.

[72] H. A. Kramers. Zur Struktur der Multiplett-S-Zustunde in zweiatomigen Molekulen. I. Zeitschrift fur Phys., 53(5-6):422-428, may 1929. ISSN 1434-6001. doi: 10.1007/BF01347762. URL http://link. springer.com/10.1007/BF01347762.

[73] E. W. Kuipers, M. G. Tenner, A. W. Kleyn, and S. Stolte. Observation of steric effects in gassurface scattering. Nature, 334(6181):420-422, aug 1988. ISSN 0028-0836. doi: 10.1038/334420a0. URL http://www . nature.com/nature/journal/v334/n6181/abs/334420a0.html.

[74] V. Kumarappan, S. S. Viftrup, L. Holmegaard, C. Z. Bisgaard, and H. Stapelfeldt. Aligning molecules with long or short laser pulses. Phys. Scr., 76(3):C63-C68, sep 2007. ISSN 0031-8949. doi: 10.1088/0031-8949/76/3/N09. URL http://stacks.iop.org/1402-4896/76/i=3/a=N09?key= crossref.f1e752da11cb6abb0ce2901fc6306819.

[75] O. Lahav, L. Levi, I. Orr, R. A. Nemirovsky, J. Nemirovsky, I. Kaminer, M. Segev, and O. Cohen. Long-lived waveguides and sound-wave generation by laser filamentation. Phys. Rev. A, 90 (2):021801, aug 2014. ISSN 1050-2947. doi: 10.1103/PhysRevA. 90.021801. URL http://link. aps .org/doi/10.1103/PhysRevA.90. 021801 .

[76] M. Lapert, S. Guérin, and D. Sugny. Field-free quantum cogwheel by shaping of rotational wave packets. Phys. Rev. A, 83(1):013403, jan 2011. ISSN 1050-2947. doi: 10.1103/PhysRevA.83.013403. URL http://link.aps.org/doi/10.1103/PhysRevA.83.013403.

[77] J. J. Larsen, K. Hald, N. Bjerre, H. Stapelfeldt, and T. Seideman. Three Dimensional Alignment of Molecules Using Elliptically Polarized Laser Fields. Phys. Rev. Lett., 85(12):2470-2473, sep 2000. ISSN 0031-9007. doi: 10.1103/PhysRevLett.85.2470. URL http: //link.aps.org/doi/10.1103/PhysRevLett.85.2470.

[78] K. F. Lee, D. M. Villeneuve, P. B. Corkum, and E. A. Shapiro. Phase Control of Rotational Wave Packets and Quantum Information. Phys. Rev. Lett., 93(23):233601, nov 2004. ISSN 0031-9007. doi: 10.1103/ PhysRevLett.93.233601. URL http://link.aps.org/doi/10.1103/ PhysRevLett.93.233601. 
[79] M. Leibscher, I. S. Averbukh, and H. Rabitz. Molecular Alignment by Trains of Short Laser Pulses. Phys. Rev. Lett., 90(21):213001, may 2003. ISSN 0031-9007. doi: 10.1103/PhysRevLett.90.213001. URL http://link.aps.org/doi/10.1103/PhysRevLett.90.213001.

[80] C. Leichtle, I. S. Averbukh, and W. P. Schleich. Generic Structure of Multilevel Quantum Beats. Phys. Rev. Lett., 77(19):3999-4002, nov 1996. ISSN 0031-9007. doi: 10.1103/PhysRevLett.77.3999. URL http://link.aps.org/doi/10.1103/PhysRevLett.77.3999.

[81] V. S. Letokhov. Laser photoionization spectroscopy. Academic Press, 1987.

[82] B. R. Lewis, S. T. Gibson, J. S. Morrill, and M. L. Ginter. Perturbations in the 3s $\sigma$ П Rydberg states of O: Bound-bound interactions with the second $\Pi$ and $\Delta$ valence states. J. Chem. Phys., 111:186, 1999. URL http://link.aip.org/link/?JCPSA6/111/186/1.

[83] J. Li, J. T. Bahns, and W. C. Stwalley. Scheme for state-selective formation of highly rotationally excited diatomic molecules. J. Chem. Phys., 112:6255, 2000. URL http://link.aip.org/link/jcpsa6/ v112/i14/p6255/s1.

[84] H. J. Loesch and F. Stienkemeier. Effect of reagent alignment on the product state distribution in the reaction $\mathrm{Sr}+\mathrm{HF}(\mathrm{v}=1, \mathrm{j}=1) \operatorname{SrF}(\mathrm{v}$, j') + H. J. Chem. Phys., 100:740, 1994. ISSN 00219606. doi: 10.1063/ 1.466942. URL http://scitation.aip.org/content/aip/journal/ jcp/100/1/10.1063/1.466942.

[85] H. J. Loesch and F. Stienkemeier. Steric effects in total integral reaction cross sections for $\mathrm{Sr}+\mathrm{HF}(\mathrm{v}=1, \mathrm{j}=1, \mathrm{~m}=0) \mathrm{SrF}+\mathrm{H}$. J. Chem. Phys., 100(6):4308, 1994. ISSN 00219606. doi: 10.1063/1.466312. URL http://scitation.aip.org/content/aip/journal/jcp/100/ 6/10.1063/1.466312.

[86] H. Maeda. Microwave Manipulation of an Atomic Electron in a Classical Orbit. Science (80-. )., 307(5716):1757-1760, mar 2005. ISSN 00368075. doi: 10.1126/science.1108470. URL http://www. sciencemag. org/cgi/doi/10.1126/science.1108470.

[87] G. Marcelin and P. R. Brooks. Steric hindrance in potassium atomoriented molecule reactions. Methyl iodide and tert-butyl iodide. $J$. Am. Chem. Soc., 97(7):1710-1715, apr 1975. ISSN 0002-7863. doi: 10. 
1021/ja00840a015. URL http://pubs.acs.org/doi/abs/10.1021/ ja00840a015.

[88] J. M. L. Martin. Basis set convergence study of the atomization energy, geometry, and anharmonic force field of SO[sub 2]: The importance of inner polarization functions. J. Chem. Phys., 108(7):2791, 1998. ISSN 00219606. doi: 10.1063/1.475670. URL http://link.aip.org/link/ JCPSA6/v108/i7/p2791/s1\{\&\}Agg=doi.

[89] L. Martinsson, P.-E. Bengtsson, M. Alden, S. Kroll, and J. Bonamy. A test of different rotational Raman linewidth models: Accuracy of rotational coherent anti-Stokes Raman scattering thermometry in nitrogen from 295 to 1850 K. J. Chem. Phys., 99(4):2466, 1993. ISSN 00219606. doi: 10.1063/1.466197. URL http://scitation.aip.org/ content/aip/journal/jcp/99/4/10.1063/1.466197.

[90] J. Miller, S. Roy, and M. Slipchenko. Single-shot gas-phase thermometry using pure-rotational hybrid femtosecond/picosecond coherent anti-Stokes Raman scattering. Opt. Express, 19(16):1562715640, 2011. doi: dx.doi.org/10.1364/OE.19.015627. URL http: //www.opticsinfobase.org/abstract.cfm?uri=oe-19-16-15627.

[91] S. L. Miller and C. H. Townes. The Microwave Absorption Spectum of $\left(\mathrm{O}^{\wedge}\{16\}\right)_{-}\{2\}$ and $\mathrm{O}^{\wedge}\{16\} \mathrm{O}^{\wedge}\{17\}$. Phys. Rev., 90(4):537-541, may 1953. ISSN 0031-899X. doi: 10.1103/PhysRev.90.537. URL http: //link.aps.org/doi/10.1103/PhysRev.90.537.

[92] G. Millot, R. Saint-Loup, J. Santos, R. Chaux, H. Berger, and J. Bonamy. Collisional effects in the stimulated Raman Q branch of O2 and O2N2. J. Chem. Phys., 96(2):961, 1992. ISSN 00219606. doi: 10.1063/1.462116. URL http://link.aip.org/link/JCPSA6/ v96/i2/p961/s1\{\&\}Agg=doi.

[93] K. Miyazaki, T. Shimizu, and D. Normand. Femtosecond-laserinduced alignment in Coulomb explosion of N 2. J. Phys. B At. Mol. Opt. Phys., 37(4):753-761, feb 2004. ISSN 0953-4075. doi: 10.1088/ 0953-4075/37/4/003. URL http://stacks . iop.org/0953-4075/37/ $i=4 / a=003$ ?key=crossref $.5 \mathrm{ae} 0 \mathrm{ae} 8 \mathrm{~d} 68215025 \mathrm{~d} 34 \mathrm{~d} 23 \mathrm{f} 97 \mathrm{~b} 4 \mathrm{f} 23 \mathrm{~d} 8$

[94] Y. Ohshima and H. Hasegawa. Coherent rotational excitation by intense nonresonant laser fields. Int. Rev. Phys. Chem., 29(4):619663, oct 2010. ISSN 0144-235X. doi: 10.1080/0144235X.2010. 
511769. URL http://www.tandfonline.com/doi/abs/10.1080/ 0144235X.2010.511769.

[95] D. H. Parker and R. B. Bernstein. Oriented Molecule Beams Via the Electrostatic Hexapole: Preparation, Characterization, and Reactive Scattering. Annu. Rev. Phys. Chem., 40(1):561-595, oct 1989. ISSN 0066-426X. doi: 10.1146/annurev.pc.40.100189.003021. URL http://www . annualreviews.org/doi/abs/10.1146/annurev . pc.40.100189.003021.

[96] J. Parker and C. R. Stroud. Rydberg Wave Packets and the Classical Limit. Phys. Scr., T12:70-75, jan 1986. ISSN 0031-8949. doi: 10.1088/0031-8949/1986/T12/010. URL http://stacks.iop.org/1402-4896/1986/i=T12/a=010?key= crossref.480d04b9e4254d80dd3a44ffef5c3450.

[97] D. Pentlehner, J. H. Nielsen, A. Slenczka, K. Mølmer, and H. Stapelfeldt. Impulsive Laser Induced Alignment of Molecules Dissolved in Helium Nanodroplets. Phys. Rev. Lett., 110(9):093002, 2013. ISSN 0031-9007. doi: 10.1103/PhysRevLett.110.093002. URL http://link.aps.org/doi/10.1103/PhysRevLett.110.093002.

[98] J. C. Polanyi. Mechanism of Rotational Relaxation. J. Chem. Phys., 56(4):1563, 1972. ISSN 00219606. doi: 10.1063/1. 1677406. URL http://scitation.aip.org/content/aip/journal/ jcp/56/4/10.1063/1.1677406.

[99] O. L. Polyansky. Water on the Sun: Line Assignments Based on Variational Calculations. Science (80-. )., 277(5324):346-348, jul 1997. ISSN 00368075. doi: 10.1126/science.277.5324.346. URL http://www. sciencemag.org/cgi/doi/10.1126/science.277.5324.346.

[100] J. H. Posthumus, J. Plumridge, M. K. Thomas, K. Codling, L. J. Frasinski, a. J. Langley, and P. F. Taday. Dynamic and geometric laser-induced alignment of molecules in intense laser fields. J. Phys. B At. Mol. Opt. Phys., 31(13):L553L562, jul 1998. ISSN 0953-4075. doi: 10.1088/0953-4075/31/ 13/002. URL http://stacks . iop.org/0953-4075/31/i=13/a=002? key=crossref . $6 \mathrm{fa} 8408 \mathrm{a} 61 \mathrm{df} 580 \mathrm{~d} 2 \mathrm{a} 187359 \mathrm{a} 2 \mathrm{a} 34 \mathrm{c} 70$.

[101] S. Purcell and P. Barker. Tailoring the Optical Dipole Force for Molecules by Field-Induced Alignment. Phys. Rev. Lett., 103(15): 
153001, oct 2009. ISSN 0031-9007. doi: 10.1103/PhysRevLett.103. 153001. URL http://link.aps.org/doi/10.1103/PhysRevLett. 103.153001

[102] S. Ramakrishna and T. Seideman. Dissipative dynamics of laser induced nonadiabatic molecular alignment. J. Chem. Phys., 124(3): 034101, jan 2006. ISSN 0021-9606. doi: 10.1063/1.2130708. URL http://www.ncbi.nlm.nih.gov/pubmed/16438561.

[103] M. Renard, E. Hertz, B. Lavorel, and O. Faucher. Controlling groundstate rotational dynamics of molecules by shaped femtosecond laser pulses. Phys. Rev. A, 69(4):043401, apr 2004. ISSN 1050-2947. doi: 10.1103/PhysRevA.69.043401. URL http://link.aps.org/doi/10. 1103/PhysRevA.69.043401.

[104] F. Rosca-Pruna and M. J. J. Vrakking. Revival structures in picosecond laser-induced alignment of I[sub 2] molecules. II. Numerical modeling. J. Chem. Phys., 116(15):6579, 2002. ISSN 00219606. doi: 10.1063/1.1458538. URL http://scitation.aip.org/content/ aip/journal/jcp/116/15/10.1063/1.1458538.

[105] A. Rouzée, S. Guérin, O. Faucher, and B. Lavorel. Field-free molecular alignment of asymmetric top molecules using elliptically polarized laser pulses. Phys. Rev. A, 77(4):043412, apr 2008. ISSN 10502947. doi: 10.1103/PhysRevA.77.043412. URL http://link.aps. org/doi/10.1103/PhysRevA.77.043412.

[106] W. Schippers, E. Gershnabel, J. Burgmeier, O. Katz, U. Willer, I. S. Averbukh, Y. Silberberg, and W. Schade. Stimulated Raman rotational photoacoustic spectroscopy using a quartz tuning fork and femtosecond excitation. Appl. Phys. B, 105(2):203-211, nov 2011. ISSN 0946-2171. doi: 10.1007/s00340-011-4725-7. URL http://link.springer.com/10.1007/s00340-011-4725-7.

[107] R. Schlapp. Fine Structure in the $3 \Sigma$ Ground State of the Oxygen Molecule, and the Rotational Intensity Distribution in the Atmospheric Oxygen Band. Phys. Rev., 51(5):342-345, mar 1937. ISSN 0031-899X. doi: 10.1103/PhysRev.51.342. URL http://link.aps. org/doi/10.1103/PhysRev.51.342.

[108] G. Scoles. Atomic and Molecular Beam Methods. Number v. 1 in Atomic and Molecular Beam Methods. Oxford University Press, 1988. 
ISBN 9780195042801. URL https://books.google.ca/books?id= uEeyQgAACAAJ.

[109] T. Seeger, J. Kiefer, A. Leipertz, B. D. Patterson, C. J. Kliewer, and T. B. Settersten. Picosecond time-resolved pure-rotational coherent anti-Stokes Raman spectroscopy for N_2 thermometry. Opt. Lett., 34(23):3755, dec 2009. ISSN 0146-9592. doi: 10.1364/OL. 34.003755. URL https://www.osapublishing.org/abstract.cfm? URI $=01-34-23-3755$.

[110] T. Seideman. Rotational excitation and molecular alignment in intense laser fields. J. Chem. Phys., 103(18):7887, 1995. ISSN 00219606. doi: 10.1063/1.470206. URL http://scitation.aip.org/content/aip/ journal/jcp/103/18/10.1063/1.470206.

[111] T. Seideman. Revival Structure of Aligned Rotational Wave Packets. Phys. Rev. Lett., 83(24):4971-4974, dec 1999. ISSN 0031-9007. doi: 10.1103/PhysRevLett.83.4971. URL http://link.aps.org/doi/10. 1103/PhysRevLett.83.4971.

[112] T. Seideman. On the dynamics of rotationally broad, spatially aligned wave packets. J. Chem. Phys., 115(13):5965, 2001. ISSN 00219606. doi: 10.1063/1.1400131. URL http://scitation.aip.org/content/ aip/journal/jcp/115/13/10.1063/1.1400131.

[113] T. Seideman and E. Hamilton. Nonadiabatic Alignment by Intense Pulses. Concepts, Theory, and Directions. Adv. At. Mol. Opt. Phys., 52:289-329, 2005. ISSN 1049250X. doi: 10.1016/S1049-250X(05) 52006-8. URL http://wWw.sciencedirect.com/science/article/ pii/S1049250X05520068.

[114] G. S. Settles. Schlieren and Shadowgraph Techniques. Springer Berlin Heidelberg, Berlin, Heidelberg, 2001. ISBN 978-3-642-63034-7. doi: 10.1007/978-3-642-56640-0. URL http://link.springer.com/10. 1007/978-3-642-56640-0.

[115] E. A. Shapiro, M. Spanner, and M. Y. Ivanov. Quantum logic in coarse grained control of wavepackets. J. Mod. Opt., 52(6):897-915, apr 2005. ISSN 0950-0340. doi: 10.1080/09500340512331313944. URL http:// Www.tandfonline.com/doi/abs/10.1080/09500340512331313944.

[116] D. Shreenivas, A. Lee, N. Walter, D. Sampayo, S. Bennett, and T. Seideman. Intense Laser Alignment As a Route to Control of Surface 
Reactions. J. Phys. Chem. A, 114(18):5674-5681, may 2010. ISSN 1089-5639. doi: 10.1021/jp9120113. URL http://pubs.acs.org/ doi/abs/10.1021/jp9120113.

[117] A. Slenczka, B. Friedrich, and D. Herschbach. Pendular alignment of paramagnetic molecules in uniform magnetic fields. Phys. Rev. Lett., 72(12):1806-1809, mar 1994. ISSN 0031-9007. doi: 10.1103/PhysRevLett.72.1806. URL http://link.aps.org/doi/10. 1103/PhysRevLett.72.1806.

[118] C. Smeenk, L. Arissian, a. Staudte, D. M. Villeneuve, and P. B. Corkum. Momentum space tomographic imaging of photoelectrons. J. Phys. B At. Mol. Opt. Phys., 42(18):185402 (5p, 2009. ISSN 09534075. doi: 10.1088/0953-4075/42/18/185402. URL http://stacks. iop.org/0953-4075/42/185402.

[119] H. Stapelfeldt and T. Seideman. Colloquium : Aligning molecules with strong laser pulses. Rev. Mod. Phys., 75(2):543-557, apr 2003. ISSN 0034-6861. doi: 10.1103/RevModPhys.75.543. URL http:// link.aps.org/doi/10.1103/RevModPhys.75.543.

[120] U. Steinitz, Y. Prior, and I. S. Averbukh. Laser-Induced Gas Vortices. Phys. Rev. Lett., 109(3):033001, jul 2012. ISSN 0031-9007. doi: 10. 1103/PhysRevLett.109.033001. URL http://link. aps . org/doi/10. 1103/PhysRevLett.109.033001.

[121] M. Strekalov. Evaluation of the contribution of elastic dephasing processes in linewidths. Chem. Phys. Lett., 332(5-6):525-530, dec 2000. ISSN 00092614. doi: 10.1016/S0009-2614(00)01310-5. URL http: //linkinghub.elsevier.com/retrieve/pii/S0009261400013105.

[122] A. Sur, C. V. Ramana, W. A. Chupka, and S. D. Colson. Rydbergvalence interactions in the П states of O. J. Chem. Phys., 84:69, 1986. URL http://link.aip.org/link/?JCPSA6/84/69/1.

[123] A. Thumann, M. Schenk, J. Jonuscheit, T. Seeger, and A. Leipertz. Simultaneous temperature and relative nitrogenoxygen concentration measurements in air with pure rotational coherent anti-Stokes Raman scattering for temperatures to as high as $2050 \mathrm{~K}$. Appl. Opt., 36(15):3500, may 1997. ISSN 0003-6935. doi: 10.1364/AO. 36.003500. URL https://www.osapublishing.org/abstract.cfm? URI $=$ ao $-36-15-3500$. 
[124] J. P. Toennies and A. F. Vilesov. Superfluid Helium Droplets: A Uniquely Cold Nanomatrix for Molecules and Molecular Complexes. Angew. Chemie Int. Ed., 43(20):2622-2648, may 2004. ISSN 14337851. doi: 10.1002/anie.200300611. URL http://doi.wiley.com/ 10.1002/anie.200300611.

[125] T. V. Tscherbul, Y. V. Suleimanov, V. Aquilanti, and R. V. Krems. Magnetic field modification of ultracold moleculemolecule collisions. New J. Phys., 11(5):055021, may 2009. ISSN 1367-2630. doi: 10.1088/1367-2630/11/5/055021. URL http://stacks.iop.org/1367-2630/11/i=5/a=055021?key= crossref.78f3d187fdeb55ea8b438fd19f2c3de8.

[126] J. Ullrich, R. Moshammer, A. Dorn, R. D rner, L. P. H. Schmidt, and H. Schmidt-B cking. Recoil-ion and electron momentum spectroscopy: reaction-microscopes. Reports Prog. Phys., 66(9):1463-1545, sep 2003. ISSN 0034-4885. doi: 10.1088/0034-4885/66/9/203. URL http:// stacks.iop.org $/ 0034-4885 / 66 / i=9 / a=203$.

[127] J. G. Underwood, B. J. Sussman, and A. Stolow. Field-Free Three Dimensional Molecular Axis Alignment. Phys. Rev. Lett., 94(14):143002, apr 2005. ISSN 0031-9007. doi: 10.1103/PhysRevLett.94.143002. URL http://link.aps.org/doi/10.1103/PhysRevLett.94.143002.

[128] J. G. Underwood, I. Procino, L. Christiansen, J. Maurer, and H. Stapelfeldt. Velocity map imaging with non-uniform detection: Quantitative molecular axis alignment measurements via Coulomb explosion imaging. Rev. Sci. Instrum., 86(7):073101, jul 2015. ISSN 0034-6748. doi: 10.1063/1.4922137. URL http://scitation.aip. org/content/aip/journal/rsi/86/7/10.1063/1.4922137.

[129] T. Vieillard, F. Chaussard, F. Billard, D. Sugny, O. Faucher, S. Ivanov, J.-M. Hartmann, C. Boulet, and B. Lavorel. Field-free molecular alignment for probing collisional relaxation dynamics. Phys. Rev. A, 87(2): 023409, feb 2013. ISSN 1050-2947. doi: 10.1103/PhysRevA.87.023409. URL http://link. aps .org/doi/10.1103/PhysRevA.87.023409.

[130] D. M. Villeneuve, S. A. Aseyev, P. Dietrich, M. Spanner, M. Y. Ivanov, and P. B. Corkum. Forced Molecular Rotation in an Optical Centrifuge. Phys. Rev. Lett., 85(3):542-545, jul 2000. ISSN 0031-9007. doi: 10.1103/PhysRevLett.85.542. URL http://link.aps.org/doi/ 10.1103/PhysRevLett.85.542. 
[131] N. V. Vitanov and B. Girard. Adiabatic excitation of rotational ladder by chirped laser pulses. Phys. Rev. A, 69(3):033409, mar 2004. ISSN 1050-2947. doi: 10.1103/PhysRevA.69.033409. URL http://link. aps.org/doi/10.1103/PhysRevA.69.033409.

[132] M. J. J. Vrakking. An iterative procedure for the inversion of two-dimensional ion/photoelectron imaging experiments. Rev. Sci. Instrum., 72(11):4084, 2001. ISSN 00346748. doi: 10.1063/1. 1406923. URL http://scitation.aip.org/content/aip/journal/ rsi/72/11/10.1063/1.1406923.

[133] J. K. Wahlstrand, N. Jhajj, E. W. Rosenthal, S. Zahedpour, and H. M. Milchberg. Direct imaging of the acoustic waves generated by femtosecond filaments in air. Opt. Lett., 39(5):1290, mar 2014. ISSN 0146-9592. doi: 10.1364/OL.39.001290. URL https://www. osapublishing.org/abstract.cfm?URI=ol-39-5-1290

[134] C. M. Western. PGOPHER, 2013. URL http://pgopher.chm.bris. ac.uk.

[135] M. G. White and R. J. Beuhler. State-resolved dynamics of oxygen atom recombination on polycrystalline Ag. J. Chem. Phys., 120(5):2445, 2004. ISSN 00219606. doi: 10.1063/1.1637333. URL http://scitation.aip.org/content/aip/journal/jcp/120/ 5/10.1063/1.1637333

[136] J. A. Yeazell and C. R. Stroud Jr. Observation of spatially localized atomic electron wave packets. Phys. Rev. Lett., 60(15):1494-1497, apr 1988. ISSN 0031-9007. doi: 10.1103/PhysRevLett.60.1494. URL http://link.aps.org/doi/10.1103/PhysRevLett.60.1494.

[137] S. Yu, C. E. Miller, B. J. Drouin, and H. S. P. Muller. High resolution spectral analysis of oxygen. I. Isotopically invariant Dunham fit for the $\mathrm{X}[\sup 3] \Sigma[$ sub g] [sup ], a1 $\Delta \mathrm{g}, \mathrm{b}[\sup 1] \Sigma[$ sub g] [sup +] states. J. Chem. Phys., 137(2):024304, 2012. ISSN 00219606. doi: 10.1063/1.4719170. URL http://scitation.aip.org/content/aip/journal/jcp/137/ 2/10.1063/1.4719170.

[138] L. Yuan, S. W. Teitelbaum, A. Robinson, and A. S. Mullin. Dynamics of molecules in extreme rotational states. Proc. Natl. Acad. Sci., 108(17):6872-6877, apr 2011. ISSN 0027-8424. doi: 10.1073/pnas. 1018669108. URL http://www.pnas.org/cgi/doi/10.1073/pnas. 1018669108 
[139] L. Yuan, C. Toro, M. Bell, and A. S. Mullin. Spectroscopy of molecules in very high rotational states using an optical centrifuge. Faraday Discuss., 150:101-111, 2011. URL http://pubs.rsc.org/en/content/ articlehtml/2011/fd/c0fd00021c.

[140] S. Yun and C. Nam. Molecular quantum rotors in gyroscopic motion with a nonspreading rotational wave packet. Phys. Rev. A, 87 (3):032520, mar 2013. ISSN 1050-2947. doi: 10.1103/PhysRevA. 87.032520. URL http://link.aps.org/doi/10.1103/PhysRevA.87. 032520 .

[141] S. Zahedpour, J. K. Wahlstrand, and H. M. Milchberg. Quantum Control of Molecular Gas Hydrodynamics. Phys. Rev. Lett., 112 (April):143601, 2014. ISSN 0031-9007. doi: 10.1103/PhysRevLett.112. 143601. URL http://link.aps.org/doi/10.1103/PhysRevLett. 112.143601

[142] S. Zhdanovich, A. A. Milner, C. Bloomquist, J. Floß, I. S. Averbukh, J. W. Hepburn, and V. Milner. Control of Molecular Rotation with a Chiral Train of Ultrashort Pulses. Phys. Rev. Lett., 107(24): 243004, dec 2011. ISSN 0031-9007. doi: 10.1103/PhysRevLett.107. 243004. URL http://link.aps.org/doi/10.1103/PhysRevLett. 107.243004

[143] S. Zhdanovich, C. Bloomquist, J. Floß, I. S. Averbukh, J. W. Hepburn, and V. Milner. Quantum Resonances in Selective Rotational Excitation of Molecules with a Sequence of Ultrashort Laser Pulses. Phys. Rev. Lett., 109(4):043003, jul 2012. ISSN 0031-9007. doi: 10.1103/PhysRevLett.109.043003. URL http://link.aps.org/doi/ 10.1103/PhysRevLett.109.043003. 